Maria Chlouveraki

\title{
BLOCKS AND FAMILIES \\ FOR CYCLOTOMIC HECKE ALGEBRAS
}




\section{Preface}

This book contains a thorough study of symmetric algebras, covering topics such as block theory, representation theory and Clifford theory. It can also serve as an introduction to the Hecke algebras of complex reflection groups. Its aim is the study of the blocks and the determination of the families of characters of the cyclotomic Hecke algebras associated to complex reflection groups.

I would like to thank my thesis' advisor, Michel Broué, for his advice. These Springer Lecture Notes were, after all, his idea. I am grateful to Jean Michel for his help with the realization and presentation of the programming part. I would also like to thank Gunter Malle for his suggestion that I generalize my results on Hecke algebras, which led to the notion of "essential algebras". Finally, I express my thanks to Cédric Bonnafé, Meinolf Geck, Nicolas Jacon, Raphaël Rouquier and Jacques Thévenaz for their useful comments. 


\section{Introduction}

The finite groups of matrices with coefficients in $\mathbb{Q}$ generated by reflections, known as Weyl groups, are a fundamental building block in the classification of semisimple complex Lie algebras and Lie groups, as well as semisimple algebraic groups over arbitrary algebraically closed fields. They are also a foundation for many other significant mathematical theories, including braid groups and Hecke algebras.

The Weyl groups are particular cases of complex reflection groups, finite groups of matrices with coefficients in a finite abelian extension of $\mathbb{Q}$ generated by "pseudo-reflections" (elements whose vector space of fixed points is a hyperplane) - if the coefficients belong to $\mathbb{R}$, then these are the finite Coxeter groups.

The work of Lusztig on the irreducible characters of reductive groups over finite fields has displayed the important role of the "families of characters" of the Weyl groups concerned. However, only recently was it realized that it would be of great interest to generalize the notion of families of characters to the complex reflection groups, or more precisely to the cyclotomic Hecke algebras associated to complex reflection groups.

On the one hand, the complex reflection groups and their associated cyclotomic Hecke algebras appear naturally in the classification of the "cyclotomic Harish-Chandra series" of the characters of the finite reductive groups, generalizing the role of the Weyl group and its traditional Hecke algebra in the principal series. Since the families of characters of the Weyl group play an essential role in the definition of the families of unipotent characters of the corresponding finite reductive group (cf. [44]), we can hope that the families of characters of the cyclotomic Hecke algebras play a key role in the organization of families of unipotent characters more generally.

On the other hand, for some complex reflection groups (non-Coxeter) $W$, some data have been gathered which seem to indicate that behind the group $W$, there exists another mysterious object - the Spets (cf. [20], [51]) - that could play the role of the "series of finite reductive groups with Weyl group $W$ ". In some cases, one can define the unipotent characters of the Spets, 
which are controlled by the "spetsial" Hecke algebra of $W$, a generalization of the classical Hecke algebra of the Weyl groups.

The main obstacle for this generalization is the lack of Kazhdan-Lusztig bases for the non-Coxeter complex reflection groups. However, more recent results of Gyoja ([40]) and Rouquier ([57]) have made possible the definition of a substitute for families of characters which can be applied to all complex reflection groups. Gyoja has shown (case by case) that the partition into " $p$-blocks" of the Iwahori-Hecke algebra of a Weyl group $W$ coincides with the partition into families, when $p$ is the unique bad prime number for $W$. Later, Rouquier proved that the families of characters of a Weyl group $W$ are exactly the blocks of characters of the Iwahori-Hecke algebra of $W$ over a suitable coefficient ring, the "Rouquier ring".

Broué, Malle and Rouquier (cf. [21]) have shown that we can define the generic Hecke algebra $\mathcal{H}(W)$ associated to a complex reflection group $W$ as a quotient of the group algebra of the braid group of $W$. The algebra $\mathcal{H}(W)$ is an algebra over a Laurent polynomial ring in a set of indeterminates $\mathbf{v}=\left(v_{i}\right)_{0 \leq i \leq m}$ whose cardinality $m$ depends on the group $W$. A cyclotomic Hecke algebra is an algebra obtained from $\mathcal{H}(W)$ via a specialization of the form $v_{i} \mapsto y^{n_{i}}$, where $y$ is an indeterminate and $n_{i} \in \mathbb{Z}$ for all $i=0,1, \ldots, m$. The blocks of a cyclotomic Hecke algebra over the Rouquier ring are the Rouquier blocks of the cyclotomic Hecke algebra. Thus, the Rouquier blocks generalize the notion of the families of characters to all complex reflection groups.

In [18], Broué and Kim presented an algorithm for the determination of the Rouquier blocks of the cyclotomic Hecke algebras of the groups $G(d, 1, r)$ and $G(e, e, r)$. Later, Kim (cf. [41]) generalized this algorithm to include all the groups of the infinite series $G(d e, e, r)$. However, it was realized recently that their algorithm does not work in general, unless $d$ is a power of a prime number. Moreover, the Rouquier blocks of the "spetsial" cyclotomic Hecke algebra of many exceptional irreducible complex reflection groups have been calculated by Malle and Rouquier in [52]. In this book, we correct and complete the determination of the Rouquier blocks for all cyclotomic Hecke algebras and all complex reflection groups.

The key in our study of the Rouquier blocks has been the proof of the fact that they have the property of "semi-continuity" (the name is due to C. Bonnafé). Every complex reflection group $W$ determines some numerical data, which in turn determine the "essential" hyperplanes for $W$. To each essential hyperplane $H$, we can associate a partition $\mathcal{B}(H)$ of the set of irreducible characters of $W$ into blocks. Given a cyclotomic specialization $v_{i} \mapsto y^{n_{i}}$, the Rouquier blocks of the corresponding cyclotomic Hecke algebra depend only on which essential hyperplanes the integers $n_{i}$ belong to. In particular, they 
are unions of the blocks associated with the essential hyperplanes to which the integers $n_{i}$ belong and they are minimal with respect to that property.

The property of semi-continuity also appears in works on Kazhdan-Lusztig cells (cf. [10], [9], [39]) and on Cherednik algebras (cf. [37]). The common appearance of this as yet unexplained phenomenon implies a connection between these structures and the Rouquier blocks, for which the reason is not yet apparent, but promises to be fruitful when explored thoroughly. In particular, due to the known relation between Kazhdan-Lusztig cells and families of characters for Coxeter groups, this could be an indication of the existence of Kazhdan-Lusztig bases for the (non-Coxeter) complex reflection groups.

Another indication of this fact comes from the determination of the Rouquier blocks of the cyclotomic Hecke algebras of all complex reflection groups, obtained in the last chapter of this book with the use of the theory of "essential hyperplanes". In the case of the Weyl groups and their usual Hecke algebra, Lusztig attaches to every irreducible character two integers, denoted by $a$ and $A$, and shows (cf. [45], 3.3 and 3.4) that they are constant on the families. In an analogous way, we can define integers $a$ and $A$ attached to every irreducible character of a cyclotomic Hecke algebra of a complex reflection group. Using the classification of the Rouquier blocks, it has been proved that the integers $a$ and $A$ are constant on the "families of characters" of the cyclotomic Hecke algebras of all complex reflection groups (see end of Chapter 4).

The first chapter of this book is dedicated to commutative algebra. The need for the results presented in this chapter (some of them are well-known, but others are completely new) arises form the fact that when we are working on Hecke algebras of complex reflection groups, we work over integrally closed rings, which are not necessarily unique factorization domains.

In the second chapter, we present some classical results of block theory and representation theory of symmetric algebras. We see that the Schur elements associated to the irreducible characters of a symmetric algebra play a crucial role in the determination of its blocks. Moreover, we generalize the results known as "Clifford theory" (cf., for example, [28]), which determine the blocks of certain subalgebras of symmetric algebras, to the case of "twisted symmetric algebras of finite groups". Finally, we give a new criterion for a symmetric algebra to be split semisimple.

In the third chapter, we introduce the notion of "essential algebras". These are symmetric algebras whose Schur elements have a specific form: they are products of irreducible polynomials evaluated on monomials. We obtain many results on the block theory of these algebras, which we later apply to the Hecke algebras, after we prove that they are essential in Chapter 4. In particular, we have our first encounter with the phenomenon of semi- 
continuity (see Theorem 3.3.2).

It is in the fourth chapter that we define for the first time the braid group, the generic Hecke algebra and the cyclotomic Hecke algebras associated to a complex reflection group. We show that the generic Hecke algebra of a complex reflection group is essential, by proving that its Schur elements are of the required form. Applying the results of Chapter 3, we obtain that the Rouquier blocks (i.e., the families of characters) of the cyclotomic Hecke algebras have the property of semi-continuity and only depend on some "essential" hyperplanes for the group, which are determined by the generic Hecke algebra.

In the fifth and final chapter of this book, we present the algorithms and the results of the determination of the families of characters for all irreducible complex reflection groups. The use of Clifford theory is essential, since it allows us to restrict ourselves to the study of only certain cases of complex reflection groups. The computations were made with the use of the GAP package CHEVIE (cf. [36]) for the exceptional irreducible complex reflection groups, whereas combinatorial methods were applied to the groups of the infinite series. In particular, we show that the families of characters for the latter can be obtained from the families of characters of the Weyl groups of type $B$, already determined by Lusztig. 


\section{Contents}

1 On Commutative Algebra $\quad \mathbf{1 0}$

1.1 Localizations . . . . . . . . . . . . . . . . . . 11

1.2 Integrally closed rings . . . . . . . . . . . . . . . . . . . . . . . . . . . . . . . . . .

1.2.1 Lifting prime ideals . . . . . . . . . . . . . . . . . . . . . . . . . . . . . . .

1.2 .2 Valuations . . . . . . . . . . . . . . . . 14

1.2.3 Discrete valuation rings and Krull rings . . . . . . . . . 17

1.3 Completions . . . . . . . . . . . . . . . . . . . . . . 19

1.4 Morphisms associated with monomials and adapted morphisms 20

1.5 Irreducibility . . . . . . . . . . . . . . . 2 27

2 On Blocks 31

2.1 Generalities . . . . . . . . . . . . . . . 32

2.1.1 Blocks and integral closure . . . . . . . . . . . 36

2.1.2 Blocks and prime ideals . . . . . . . . . . . 37

2.1.3 Blocks and quotient blocks . . . . . . . . . . . . . . . . . . . 39

2.1.4 Blocks and central characters . . . . . . . . . . . 40

2.2 Symmetric algebras . . . . . . . . . . . . . . . . . . . . 41

2.3 Twisted symmetric algebras of finite groups . . . . . . . . . 44

2.3.1 Action of $G$ on $Z \bar{A} \ldots \ldots \ldots$. . . . . . . 50

2.3.2 Multiplication of an $A$-module by an $\mathcal{O} G$-module . . . 52

2.3.3 Induction and restriction of $K A$-modules and $K \bar{A}$-modules 52

2.3.4 Blocks of $A$ and blocks of $\bar{A} \ldots \ldots . . . \ldots 58$

2.4 Representation theory of symmetric algebras . . . . . . . . 60

2.4.1 Grothendieck groups . . . . . . . . . . . . . . 60

2.4 .2 Integrality . . . . . . . . . . . . . . . . 62

2.4.3 The decomposition map . . . . . . . . . . . 63

2.4.4 A variation for Tits' deformation theorem . . . . . . 67

2.4.5 Symmetric algebras over discrete valuation rings . . . . 68 
3 On Essential algebras $\quad 73$

3.1 Generalities . . . . . . . . . . . . . . . 73

3.2 Specialization via morphisms associated with monomials . . . 76

3.3 Specialization via adapted morphisms . . . . . . . . . . 78

3.4 The $\operatorname{map} I^{n} \ldots \ldots \ldots$

4 On Hecke algebras 84

4.1 Complex reflection groups and associated braid groups . . . . 85

4.1.1 Complex reflection groups . . . . . . . . . 85

4.1.2 Braid groups associated to complex reflection groups . 86

4.2 Generic Hecke algebras . . . . . . . . . . . . . . . . . . . . 89

4.3 Cyclotomic Hecke algebras . . . . . . . . . . . . . . . . . . 94

4.3.1 Essential hyperplanes . . . . . . . . . . . . 996

4.3.2 Group algebra . . . . . . . . . . . . . . . . . 98

4.4 Rouquier blocks of the cyclotomic Hecke algebras . . . . . . . 98

4.4.1 Rouquier blocks and central morphisms . . . . . . . . . 102

4.4.2 Rouquier blocks and functions $a$ and $A$. . . . . . 102

5 On the determination of the Rouquier blocks 105

5.1 General principles . . . . . . . . . . . . . . . . 106

5.2 The exceptional irreducible complex reflection groups . . . . . 108

5.2.1 Essential hyperplanes . . . . . . . . . . . . . . . 109

5.2 .2 Algorithm . . . . . . . . . . . . . . . . 111

5.2 .3 Results . . . . . . . . . . . . . . . . . 112

5.3 The groups $G(d, 1, r) \ldots \ldots \ldots \ldots$

5.3 .1 Combinatorics . . . . . . . . . . . . 120

5.3 .2 Ariki-Koike algebras . . . . . . . . . . . . . 123

5.3.3 Rouquier blocks, charged content and residues . . . . . 124

5.3 .4 Essential hyperplanes . . . . . . . . . . . . . . 126

5.3 .5 Results . . . . . . . . . . . . . . . 126

5.4 The groups $G(2 d, 2,2) \quad \ldots \ldots \ldots \ldots \ldots$

5.4.1 Essential hyperplanes . . . . . . . . . . . . . 134

5.4 Results . . . . . . . . . . . . . . . 135

5.5 The groups $G(d e, e, r) \ldots \ldots \ldots \ldots \ldots$

5.5.1 The groups $G(d e, e, r), r>2 \ldots \ldots \ldots 139$

5.5.2 The groups $G(d e, e, 2) \ldots \ldots . . \ldots 146$

6 Appendix: Clifford theory and Schur elements for the Hecke algebras of complex reflection groups $\quad 151$

6.1 The groups $G_{4}, \ldots, G_{7} \ldots \ldots \ldots \ldots$

6.2 The groups $G_{8}, \ldots, G_{15} \ldots \ldots \ldots$ 
6.3 The groups $G_{16}, \ldots, G_{22} \ldots \ldots \ldots \ldots \ldots$

6.4 The groups $G_{25}, G_{26} \ldots \ldots \ldots \ldots \ldots \ldots$

6.5 The group $G_{28}\left(" F_{4}\right.$ ") $\ldots \ldots \ldots \ldots \ldots \ldots \ldots$

6.6 The group $G_{32} \ldots \ldots \ldots \ldots \ldots \ldots \ldots \ldots$

6.7 The groups $G(d e, e, r) \ldots \ldots \ldots \ldots \ldots$

6.7.1 The groups $G(d e, e, r), r>2 \ldots \ldots \ldots$

6.7.2 The groups $G(d e, e, 2), e$ odd $\ldots \ldots \ldots \ldots$

6.7.3 The groups $G(d e, e, 2), e$ even $\ldots \ldots \ldots$

Bibliography 


\section{Chapter 1}

\section{On Commutative Algebra}

The first chapter contains some known facts and some new results on Commutative Algebra. The former are presented here without their proofs (with the exception of Theorem 1.4.1) for the convenience of the reader. They are going to be crucial in the proofs of the results of Chapters 3 and 4 .

In the first section of this chapter, we define the localization of a ring and give some main properties. The second section is dedicated on integrally closed rings. We study particular cases of integrally closed rings, such as valuation rings, discrete valuation rings and Krull rings. We use their properties in order to obtain results on Laurent polynomial rings over integrally closed rings. We state briefly some results on the completions of rings in section 1.3. In the fourth section, we introduce the notion of morphisms associated with monomials. They are morphisms which allow us to pass from a Laurent polynomial ring $A$ in $m+1$ indeterminates to a Laurent polynomial ring $B$ in $m$ indeterminates, while sending a specific monomial to 1 . Moreover, we prove (Proposition 1.4.9) that every surjective morphism from $A$ to $B$ which sends each indeterminate to a monomial is associated with a monomial. We call adapted morphisms the compositions of morphisms associated with monomials. They play a powerful role in the proof of the main results of Chapters 3 and 4 . Finally, in the last section of the first chapter, we give a criterion (Theorem 1.5.6) for a polynomial to be irreducible in a Laurent polynomial ring with coefficients in a field.

Throughout this chapter, all rings are assumed to be commutative with 1. Moreover, if $R$ is a ring and $x_{0}, x_{1}, \ldots, x_{m}$ is a set of indeterminates on $R$, then we denote by $R\left[x_{0}^{ \pm 1}, x_{1}^{ \pm 1}, \ldots, x_{m}^{ \pm 1}\right]$ the Laurent polynomial ring in $m+1$ indeterminates $R\left[x_{0}, x_{0}^{-1}, x_{1}, x_{1}^{-1}, \ldots, x_{m}, x_{m}{ }^{-1}\right]$. 


\subsection{Localizations}

Definition 1.1.1 Let $R$ be a commutative ring with 1 . We say that a subset $S$ of $R$ is a multiplicatively closed set if $0 \notin S, 1 \in S$ and every finite product of elements of $S$ belongs to $S$.

In the set $R \times S$, we introduce an equivalence relation such that $(r, s)$ is equivalent to $\left(r^{\prime}, s^{\prime}\right)$ if and only if there exists $t \in S$ such that $t\left(s^{\prime} r-s r^{\prime}\right)=0$. We denote the equivalence class of $(r, s)$ by $r / s$. The set of equivalence classes becomes a ring under the operations such that the sum and the product of $r / s$ and $r^{\prime} / s^{\prime}$ are given by $\left(s^{\prime} r+s r^{\prime}\right) / s s^{\prime}$ and $r r^{\prime} / s s^{\prime}$ respectively. We denote this ring by $S^{-1} R$ and we call it the localization of $R$ at $S$. If $S$ contains no zero divisors of $R$, then any element $r$ of $R$ can be identified with the element $r / 1$ of $S^{-1} R$ and we can regard the latter as an $R$-algebra.

Remarks:

- If $S$ is the set of all non-zero divisors of $R$, then $S^{-1} R$ is called the total quotient ring of $R$. If, moreover, $R$ is an integral domain, the total quotient ring of $R$ is the field of fractions of $R$.

- If $R$ is Notherian, then $S^{-1} R$ is Noetherian.

- If $\mathfrak{p}$ is a prime ideal of $R$, then the set $S:=R-\mathfrak{p}$ is a multiplicatively closed subset of $R$. Then the ring $S^{-1} R$ is simply denoted by $R_{\mathfrak{p}}$.

The proofs for the following well known results concerning localizations can be found in [11].

Proposition 1.1.2 Let $A$ and $B$ be two rings with multiplicative sets $S$ and $T$ respectively and $f$ an homomorphism from $A$ to $B$ such that $f(S)$ is contained in $T$. There exists a unique homomorphism $f^{\prime}$ from $S^{-1} A$ to $T^{-1} B$ such that $f^{\prime}(a / 1)=f(a) / 1$ for every $a \in A$. Let us suppose now that $T$ is contained in the multiplicatively closed set of $B$ generated by $f(S)$. If $f$ is surjective (resp. injective), then $f^{\prime}$ is also surjective (resp. injective).

Corollary 1.1.3 Let $A$ and $B$ be two rings with multiplicative sets $S$ and $T$ respectively such that $A \subseteq B$ and $S \subseteq T$. Then $S^{-1} A \subseteq T^{-1} B$.

Proposition 1.1.4 Let $A$ be a ring and $S, T$ two multiplicative sets of $A$ such that $S \subseteq T$. We have $S^{-1} A=T^{-1} A$ if and only if every prime ideal of $R$ that meets $T$ meets $S$. 
The following proposition and its corollary give us information about the ideals of the localization of a ring $R$ at a multiplicatively closed subset $S$ of $R$.

Proposition 1.1.5 Let $R$ be a ring and let $S$ be a multiplicatively closed subset of $R$. Then

1. Every ideal $\mathfrak{b}^{\prime}$ of $S^{-1} R$ is of the form $S^{-1} \mathfrak{b}$ for some ideal $\mathfrak{b}$ of $R$.

2. Let $\mathfrak{b}$ be an ideal of $R$ and let $f$ be the canonical surjection $R \rightarrow R / \mathfrak{b}$. Then $f(S)$ is a multiplicatively closed subset of $R / \mathfrak{b}$ and the homomorphism from $S^{-1} R$ to $(f(S))^{-1}(R / \mathfrak{b})$ canonically associated with $f$ is surjective with kernel $\mathfrak{b}^{\prime}=S^{-1} \mathfrak{b}$. By passing to quotients, an isomorphism between $\left(S^{-1} R\right) / \mathfrak{b}^{\prime}$ and $(f(S))^{-1}(R / \mathfrak{b})$ is defined.

3. The application $\mathfrak{b}^{\prime} \mapsto \mathfrak{b}$, restricted to the set of maximal (resp. prime) ideals of $S^{-1} R$, is an isomorphism (for the relation of inclusion) between this set and the set of maximal (resp. prime) ideals of $R$ that do not meet $S$.

4. If $\mathfrak{q}^{\prime}$ is a prime ideal of $S^{-1} R$ and $\mathfrak{q}$ is the prime ideal of $R$ such that $\mathfrak{q}^{\prime}=S^{-1} \mathfrak{q}$ (we have $\mathfrak{q} \cap S=\emptyset$ ), then there exists an isomorphism from $R_{\mathfrak{q}}$ to $\left(S^{-1} R\right)_{\mathfrak{q}^{\prime}}$ which sends $r / s$ to $(r / 1) /(s / 1)$ for $r \in R, s \in R-\mathfrak{q}$.

Corollary 1.1.6 Let $R$ be a ring, $\mathfrak{p}$ a prime ideal of $R$ and $S:=R-\mathfrak{p}$. For every ideal $\mathfrak{b}$ of $R$ which does not meet $S$, let $\mathfrak{b}^{\prime}:=\mathfrak{b} R_{\mathfrak{p}}$. Assume that $\mathfrak{b}^{\prime} \neq R_{\mathfrak{p}}$. Then

1. Let $f$ be the canonical surjection $R \rightarrow R / \mathfrak{b}$. The ring homomorphism from $R_{\mathfrak{p}}$ to $(R / \mathfrak{b})_{\mathfrak{p} / \mathfrak{b}}$ canonically associated with $f$ is surjective and its kernel is $\mathfrak{b}^{\prime}$. Thus it defines, by passing to quotients, a canonical isomorphism between $R_{\mathfrak{p}} / \mathfrak{b}^{\prime}$ and $(R / \mathfrak{b})_{\mathfrak{p} / \mathfrak{b}}$.

2. The application $\mathfrak{b}^{\prime} \mapsto \mathfrak{b}$, restricted to the set of prime ideals of $R_{\mathfrak{p}}$, is an isomorphism (for the relation of inclusion) between this set and the set of prime ideals of $R$ contained in $\mathfrak{p}$ (thus do not meet $S$ ). Therefore, $\mathfrak{p} R_{\mathfrak{p}}$ is the only maximal ideal of $R_{\mathfrak{p}}$.

3. If now $\mathfrak{b}^{\prime}$ is a prime ideal of $R_{\mathfrak{p}}$, then there exists an isomorphism from $R_{\mathfrak{b}}$ to $\left(R_{\mathfrak{p}}\right)_{\mathfrak{b}^{\prime}}$ which sends $r / s$ to $(r / 1) /(s / 1)$ for $r \in R, s \in R-\mathfrak{b}$.

The notion of localization can also be extended to the modules over the ring $R$.

Definition 1.1.7 Let $R$ be a ring and $S$ a multiplicatively closed set of $R$. If $M$ is an R-module, then we call localization of $M$ at $S$ and denote by $S^{-1} M$ the $S^{-1} R$-module $M \otimes_{R} S^{-1} R$. 


\section{$1.2 \quad$ Integrally closed rings}

Theorem-Definition 1.2.1 Let $R$ be a ring, $A$ an $R$-algebra and a an element of $A$. The following properties are equivalent:

(i) The element a is a root of a monic polynomial with coefficients in $R$.

(ii) The subalgebra $R[a]$ of $A$ is an $R$-module of finite type.

(iii) There exists a faithful $R[a]$-module which is an $R$-module of finite type.

If $a \in A$ verifies the conditions above, we say that it is integral over $R$.

Definition 1.2.2 Let $R$ be a ring and $A$ an $R$-algebra. The set of all elements of $A$ that are integral over $R$ is an $R$-subalgebra of $A$ containing $R$; it is called the integral closure of $R$ in $A$. We say that $R$ is integrally closed in $A$, if $R$ is an integral domain and if it coincides with its integral closure in $A$. If now $R$ is an integral domain and $F$ is its field of fractions, then the integral closure of $R$ in $F$ is named simply the integral closure of $R$, and if $R$ is integrally closed in $F$, then $R$ is said to be integrally closed.

The following proposition ([12], $\S 1$, Prop.13) implies that transfer theorem holds for integrally closed rings (corollary 1.2.4).

Proposition 1.2.3 If $R$ is an integral domain, let us denote by $\bar{R}$ the integral closure of $R$. Let $x_{0}, \ldots, x_{m}$ be a set of indeterminates over $R$. Then the integral closure of $R\left[x_{0}, \ldots, x_{m}\right]$ is $\bar{R}\left[x_{0}, \ldots, x_{m}\right]$.

Corollary 1.2.4 Let $R$ be an integral domain. Then $R\left[x_{0}, \ldots, x_{m}\right]$ is integrally closed if and only if $R$ is integrally closed.

Corollary 1.2.5 If $K$ is a field, then every polynomial ring $K\left[x_{0}, \ldots, x_{m}\right]$ is integrally closed.

The next proposition ([12], §1, Prop.16) along with its corollaries treats the integral closures of localizations of rings.

Proposition 1.2.6 Let $R$ be a ring, $A$ an $R$-algebra, $\bar{R}$ the integral closure of $R$ in $A$ and $S$ a multiplicatively closed subset of $R$ which contains no zero divisors. Then the integral closure of $S^{-1} R$ in $S^{-1} A$ is $S^{-1} \bar{R}$.

Corollary 1.2.7 Let $R$ be an integral domain, $\bar{R}$ the integral closure of $R$ and $S$ a multiplicatively closed subset of $R$. Then the integral closure of $S^{-1} R$ is $S^{-1} \bar{R}$. 
Corollary 1.2.8 If $R$ is an integrally closed domain and $S$ is a multiplicatively closed subset of $R$, then $S^{-1} R$ is also integrally closed.

Example 1.2.9 Let $K$ be a finite field extension of $\mathbb{Q}$ and $\mathbb{Z}_{K}$ the integral closure of $\mathbb{Z}$ in $K$. Obviously, the ring $\mathbb{Z}_{K}$ is integrally closed. Let $x_{0}, x_{1}, \ldots, x_{m}$ be indeterminates. Then the ring $\mathbb{Z}_{K}\left[x_{0}^{ \pm}, x_{1}^{ \pm}, \ldots, x_{m}^{ \pm}\right]$is also integrally closed.

\subsubsection{Lifting prime ideals}

Definition 1.2.10 Let $R, R^{\prime}$ be two rings and let $h: R \rightarrow R^{\prime}$ be a ring homomorphism. We say that a prime ideal $\mathfrak{a}^{\prime}$ of $R^{\prime}$ lies over a prime ideal $\mathfrak{a}$ of $R$, if $\mathfrak{a}=h^{-1}\left(\mathfrak{a}^{\prime}\right)$.

The next result is [12], $\S 2$, Proposition 2 .

Proposition 1.2.11 Let $h: R \rightarrow R^{\prime}$ be a ring homomorphism such that $R^{\prime}$ is integral over $R$. Let $\mathfrak{p}$ be a prime ideal of $R, S:=R-\mathfrak{p}$ and $\left(\mathfrak{p}_{i}^{\prime}\right)_{i \in I}$ the family of all the prime ideals of $R^{\prime}$ lying over $\mathfrak{p}$. If $S^{\prime}=\bigcap_{i \in I}\left(R^{\prime}-\mathfrak{p}_{i}^{\prime}\right)$, then $S^{-1} R^{\prime}=S^{\prime-1} R^{\prime}$.

The following corollary of Proposition 1.2.11 deals with a case we will encounter in a following chapter, where there exists a unique prime ideal lying over the prime ideal $\mathfrak{p}$ of $R$. In combination with Proposition 1.2.6, Proposition 1.2.11 implies that

Corollary 1.2.12 Let $R$ be an integral domain, $A$ an $R$-algebra, $\bar{R}$ the integral closure of $R$ in $A$. Let $\mathfrak{p}$ be a prime ideal of $R$ and $S:=R-\mathfrak{p}$. If there exists a unique prime ideal $\overline{\mathfrak{p}}$ of $\bar{R}$ lying over $\mathfrak{p}$, then the integral closure of $R_{\mathfrak{p}}$ in $S^{-1} A$ is $\bar{R}_{\overline{\mathfrak{p}}}$.

\subsubsection{Valuations}

Definition 1.2.13 Let $R$ be a ring and $\Gamma$ a totally ordered abelian group. We call valuation of $R$ with values in $\Gamma$ every application $v: R \rightarrow \Gamma \cup\{\infty\}$ which satisfies the following properties:

(V1) $v(x y)=v(x)+v(y)$ for $x \in R, y \in R$.

(V2) $v(x+y) \geq \inf (v(x), v(y))$ for $x \in R, y \in R$.

(V3) $v(1)=0$ and $v(0)=\infty$. 
In particular, if $v(x) \neq v(y)$, property (V2) gives $v(x+y)=\inf (v(x), v(y))$ for $x \in R, y \in R$. Moreover, from property (V1), we have that if $z \in R$ with $z^{n}=1$ for some integer $n \geq 1$, then $n v(z)=v\left(z^{n}\right)=v(1)=0$ and thus $v(z)=0$. Consequently, $v(-x)=v(-1)+v(x)=v(x)$ for all $x \in R$.

Now let $F$ be a field and let $v: F \rightarrow \Gamma$ be a valuation of $F$. The set $A$ of $a \in F$ such that $v(a) \geq 0$ is a local subring of $F$. Its maximal ideal $\mathfrak{m}(A)$ is the set of $a \in A$ such that $v(a)>0$. For all $a \in F-A$, we have $a^{-1} \in \mathfrak{m}(A)$. The ring $A$ is called the ring of the valuation $v$ on $F$.

We will now introduce the notion of a valuation ring. For more information about valuation rings and their properties, see [13]. Some of them will also be discussed in Chapter 2, Section 2.4.

Definition 1.2.14 Let $R$ be an integral domain contained in a field $F$. Then $R$ is a valuation ring if for all non-zero element $x \in F$, we have $x \in R$ or $x^{-1} \in R$. Consequently, $F$ is the field of fractions of $R$.

If $R$ is a valuation ring, then it has the following properties:

- It is an integrally closed local ring.

- The set of the principal ideals of $R$ is totally ordered by the relation of inclusion.

- The set of the ideals of $R$ is totally ordered by the relation of inclusion.

Let $R$ be a valuation ring and $F$ its field of fractions. Let us denote by $R^{\times}$the set of units of $R$. Then the set $\Gamma_{R}:=F^{\times} / R^{\times}$is an abelian group, totally ordered by the relation of inclusion of the corresponding principal ideals. If we denote by $v_{R}$ the canonical homomorphism of $F^{\times}$onto $\Gamma_{R}$ and set $v_{R}(0)=\infty$, then $v_{R}$ is a valuation of $F$ whose ring is $R$.

The following proposition gives a characterization of integrally closed rings in terms of valuation rings ([13], $\S 1$, Thm. 3).

Proposition 1.2.15 Let $R$ be a subring of a field $F$. The integral closure $\bar{R}$ of $R$ in $F$ is the intersection of all valuation rings in $F$ which contain $R$. Consequently, an integral domain $R$ is integrally closed if and only if it is the intersection of a family of valuation rings contained in its field of fractions.

This characterization helped us to prove the following result about integrally closed rings. 
Proposition 1.2.16 Let $R$ be an integrally closed ring and $f(x)=\sum_{i} a_{i} x^{i}$, $g(x)=\sum_{j} b_{j} x^{j}$ be two polynomials in $R[x]$. If there exists an element $c \in R$ such that all the coefficients of $f(x) g(x)$ belong to $c R$, then all the products $a_{i} b_{j}$ belong to $c R$.

Proof: Due to Proposition 1.2.15, it is enough to prove the result in the case where $R$ is a valuation ring.

From now on, let $R$ be a valuation ring. Let $v$ be a valuation of the field of fractions of $R$ such that the ring of valuation of $v$ is $R$. Let $\kappa:=$ $\inf _{i}\left(v\left(a_{i}\right)\right)$ and $\lambda:=\inf _{j}\left(v\left(b_{j}\right)\right)$. Then $\kappa+\lambda=\inf _{i, j}\left(v\left(a_{i} b_{j}\right)\right)$. We will show that $\kappa+\lambda \geq v(c)$ and thus $c$ divides all the products $a_{i} b_{j}$. Argue by contradiction and assume that $\kappa+\lambda<v(c)$. Let $a_{i_{1}}, a_{i_{2}}, \ldots, a_{i_{r}}$ with $i_{1}<i_{2}<\ldots<i_{r}$ be all the elements among the $a_{i}$ with valuation equal to $\kappa$. Respectively, let $b_{j_{1}}, b_{j_{2}}, \ldots, b_{j_{s}}$ with $j_{1}<j_{2}<\ldots<j_{s}$ be all the elements among the $b_{j}$ with valuation equal to $\lambda$. We have that $i_{1}+j_{1}<i_{m}+j_{n}, \forall(m, n) \neq(1,1)$. Therefore, the coefficient $c_{i_{1}+j_{1}}$ of $x^{i_{1}+j_{1}}$ in $f(x) g(x)$ is of the form $\left(a_{i_{1}} b_{j_{1}}+\sum\right.$ (terms with valuation $\left.\left.>\kappa+\lambda\right)\right)$ and since $v\left(a_{i_{1}} b_{j_{1}}\right) \neq v\left(\sum(\right.$ terms with valuation $\left.>\kappa+\lambda)\right)$, we obtain that

$$
v\left(c_{i_{1}+j_{1}}\right)=\inf \left(v\left(a_{i_{1}} b_{j_{1}}\right), v\left(\sum(\text { terms with valuation }>\kappa+\lambda)\right)\right)=\kappa+\lambda .
$$

However, since all the coefficients of $f(x) g(x)$ are divisible by $c$, we have that $v\left(c_{i_{1}+j_{1}}\right) \geq v(c)>\kappa+\lambda$, which is a contradiction.

The Propositions 1.2.17 and 1.2.19 derive from the one above. We will make use of the results in corollaries 1.2 .18 and 1.2 .20 in Chapter 3.

Proposition 1.2.17 Let $R$ be an integrally closed domain and let $F$ be its field of fractions. Let $\mathfrak{p}$ be a prime ideal of $R$. Then

$$
(R[x])_{\mathfrak{p} R[x]} \cap F[x]=R_{\mathfrak{p}}[x] .
$$

Proof: The inclusion $R_{\mathfrak{p}}[x] \subseteq(R[x])_{\mathfrak{p} R[x]} \cap F[x]$ is obvious. Now, let $f(x)$ be an element of $F[x]$. Then $f(x)$ can be written in the form $r(x) / \xi$, where $r(x) \in R[x]$ and $\xi \in R$. If, moreover, $f(x)$ belongs to $(R[x])_{\mathfrak{p} R[x]}$, then there exist $s(x), t(x) \in R[x]$ with $t(x) \notin \mathfrak{p} R[x]$ such that $f(x)=s(x) / t(x)$. Thus we have

$$
f(x)=\frac{r(x)}{\xi}=\frac{s(x)}{t(x)} .
$$

All the coefficients of the product $r(x) t(x)$ belong to $\xi R$. Due to Proposition 1.2.16, if $r(x)=\sum_{i} a_{i} x^{i}$ and $t(x)=\sum_{j} b_{j} x^{j}$, then all the products 
$a_{i} b_{j}$ belong to $\xi R$. Since $t(x) \notin \mathfrak{p} R[x]$, there exists $j_{0}$ such that $b_{j_{0}} \notin \mathfrak{p}$ and $a_{i} b_{j_{0}} \in \xi R, \forall i$. Consequently, $b_{j_{0}} f(x)=b_{j_{0}}(r(x) / \xi) \in R[x]$ and hence all the coefficients of $f(x)$ belong to $R_{\mathfrak{p}}$.

Corollary 1.2.18 Let $R$ be an integrally closed domain and let $F$ be its field of fractions. Let $\mathfrak{p}$ be a prime ideal of $R$. Then

$$
\begin{aligned}
& \text { 1. }\left(R\left[x, x^{-1}\right]\right)_{\mathfrak{p} R\left[x, x^{-1}\right]} \cap F\left[x, x^{-1}\right]=R_{\mathfrak{p}}\left[x, x^{-1}\right] . \\
& \text { 2. }\left(R\left[x_{0}, \ldots, x_{m}\right]\right)_{\mathfrak{p} R\left[x_{0}, \ldots, x_{m}\right]} \cap F\left[x_{0}, \ldots, x_{m}\right]=R_{\mathfrak{p}}\left[x_{0}, \ldots, x_{m}\right] . \\
& \text { 3. }\left(R\left[x_{0}^{ \pm 1}, \ldots, x_{m}^{ \pm 1}\right]\right)_{\mathfrak{p} R\left[x_{0}^{ \pm 1}, \ldots, x_{m}^{ \pm 1}\right]} \cap F\left[x_{0}^{ \pm 1}, \ldots, x_{m}^{ \pm 1}\right]=R_{\mathfrak{p}}\left[x_{0}^{ \pm 1}, \ldots, x_{m}^{ \pm 1}\right] .
\end{aligned}
$$

Proposition 1.2.19 Let $R$ be an integrally closed domain and let $F$ be its field of fractions. Let $r(x)$ and $s(x)$ be two elements of $R[x]$ such that $s(x)$ divides $r(x)$ in $F[x]$. If one of the coefficients of $s(x)$ is a unit in $R$, then $s(x)$ divides $r(x)$ in $R[x]$.

Proof: $\quad$ Since $s(x)$ divides $r(x)$ in $F[x]$, there exists an element of the form $t(x) / \xi$ with $t(x) \in R[x]$ and $\xi \in R$ such that

$$
r(x)=\frac{s(x) t(x)}{\xi}
$$

All the coefficients of the product $s(x) t(x)$ belong to $\xi R$. Due to Proposition 1.2.16, if $s(x)=\sum_{i} a_{i} x^{i}$ and $t(x)=\sum_{j} b_{j} x^{j}$, then all the products $a_{i} b_{j}$ belong to $\xi R$. By assumption, there exists $i_{0}$ such that $a_{i_{0}}$ is a unit in $R$ and $a_{i_{0}} b_{j} \in \xi R, \forall j$. Consequently, $b_{j} \in \xi R, \forall j$ and thus $t(x) / \xi \in R[x]$.

Corollary 1.2.20 Let $R$ be an integrally closed domain and let $F$ be its field of fractions. Let $r, s$ be two elements of $R\left[x_{0}^{ \pm 1}, \ldots, x_{m}^{ \pm 1}\right]$ such that $s$ divides $r$ in $F\left[x_{0}^{ \pm 1}, \ldots, x_{m}^{ \pm 1}\right]$. If one of the coefficients of $s$ is a unit in $R$, then $s$ divides $r$ in $R\left[x_{0}^{ \pm 1}, \ldots, x_{m}^{ \pm 1}\right]$.

\subsubsection{Discrete valuation rings and Krull rings}

Definition 1.2.21 Let $F$ be a field, $\Gamma$ a totally ordered abelian group and $v$ a valuation of $F$ with values in $\Gamma$. We say that the valuation $v$ is discrete, if $\Gamma$ is isomorphic to $\mathbb{Z}$.

Theorem-Definition 1.2.22 An integral domain $R$ is a discrete valuation ring, if it satisfies one of the following equivalent conditions: 
(i) $R$ is the ring of a discrete valuation.

(ii) $R$ is a local Dedekind ring.

(iii) $R$ is a local principal ideal domain.

(iv) $R$ is a Noetherian valuation ring.

By Proposition 1.2.15, integrally closed rings are intersections of valuation rings. Krull rings are essentially intersections of discrete valuation rings.

Definition 1.2.23 An integral domain $R$ is a Krull ring, if there exists a family of valuations $\left(v_{i}\right)_{i \in I}$ of the field of fractions $F$ of $R$ with the following properties:

(K1) The valuations $\left(v_{i}\right)_{i \in I}$ are discrete.

(K2) The intersection of the rings of $\left(v_{i}\right)_{i \in I}$ is $R$.

(K3) For all $x \in F^{\times}$, there exists a finite number of $i \in I$ such that $v_{i}(x) \neq 0$.

The proofs of the following results and more information about Krull rings can be found in [14], $\S 1$.

Theorem 1.2.24 Let $R$ be an integral domain and let $\operatorname{Spec}_{1}(R)$ be the set of its prime ideals of height 1 . Then $R$ is a Krull ring if and only if the following properties are satisfied:

1. For all $\mathfrak{p} \in \operatorname{Spec}_{1}(R), R_{\mathfrak{p}}$ is a discrete valuation ring.

2. $R$ is the intersection of $R_{\mathfrak{p}}$ for all $\mathfrak{p} \in \operatorname{Spec}_{1}(R)$.

3. For all $r \neq 0$ in $R$, there exists a finite number of ideals $\mathfrak{p} \in \operatorname{Spec}_{1}(R)$ such that $r \in \mathfrak{p}$.

Transfer theorem holds also for Krull rings:

Proposition 1.2.25 Let $R$ be a Krull ring, $F$ the field of fractions of $R$ and $x$ an indeterminate. Then $R[x]$ is also a Krull ring. Moreover, its prime ideals of height 1 are:

- the prime ideals of the form $\mathfrak{p} R[x]$, where $\mathfrak{p}$ is a prime ideal of height 1 of $R$,

- the prime ideals of the form $\mathfrak{m} \cap R[x]$, where $\mathfrak{m}$ is a prime ideal of $F[x]$. 
The following proposition provides us with a simple characterization of Krull rings, when they are Noetherian.

Proposition 1.2.26 Let $R$ be a Noetherian ring. Then $R$ is a Krull ring if and only if it is integrally closed.

Example 1.2.27 Let $K$ be a finite field extension of $\mathbb{Q}$ and $\mathbb{Z}_{K}$ the integral closure of $\mathbb{Z}$ in $K$. The ring $\mathbb{Z}_{K}$ is a Dedekind ring and thus Noetherian and integrally closed. Let $x_{0}, x_{1}, \ldots, x_{m}$ be indeterminates. Then the $\operatorname{ring} \mathbb{Z}_{K}\left[x_{0}^{ \pm}, x_{1}^{ \pm}, \ldots, x_{m}^{ \pm}\right]$is also Noetherian and integrally closed and thus a Krull ring.

\subsection{Completions}

For the proofs of all the following results concerning completions, the reader can refer to [55], Chapter II.

Let $I$ be an ideal of a commutative $\operatorname{ring} R$ and let $M$ be an $R$-module. We introduce a topology on $M$ such that the open sets of $M$ are unions of an arbitrary number of sets of the form $m+I^{n} M(m \in M)$. This topology is called the $I$-adic topology of $M$.

Theorem 1.3.1 If $M$ is a Noetherian $R$-module, then for any submodule $N$ of $M$, the I-adic topology of $N$ coincides with the topology of $N$ as a subspace of $M$ with the I-adic topology.

From now on, we will concentrate on semi-local rings and in particular, on Noetherian semi-local rings.

Definition 1.3.2 A ring $R$ is called semi-local, if it has only a finite number of maximal ideals. The Jacobson radical $\mathfrak{m}$ of $R$ is the intersection of the maximal ideals of $R$.

Theorem 1.3.3 Assume that $R$ is a Noetherian semi-local ring with Jacobson radical $\mathfrak{m}$ and let $\hat{R}$ be the completion of $R$ with respect to the $\mathfrak{m}$-adic topology. Then $\hat{R}$ is also a Noetherian semi-local ring and we have $R \subseteq \hat{R}$.

Theorem 1.3.4 Assume that $R$ is a Noetherian semi-local ring with Jacobson radical $\mathfrak{m}$ and that $M$ is a finitely generated $R$-module. Let $\hat{R}$ be the completion of $R$ with respect to the $\mathfrak{m}$-adic topology. Endow $M$ with the $\mathfrak{m}$ adic topology. Then $M \otimes_{R} \hat{R}$ is the completion of $M$ with respect to that topology. 
Corollary 1.3.5 Let $\mathfrak{a}$ be an ideal of a Noetherian semi-local ring $R$ with Jacobson radical $\mathfrak{m}$. Let $\hat{R}$ be the completion of $R$ with respect to the $\mathfrak{m}$-adic topology. Then the completion of $\mathfrak{a}$ is $\mathfrak{a} \hat{R}$ and $\mathfrak{a} \hat{R}$ is isomorphic to $\mathfrak{a} \otimes_{R} \hat{R}$. Furthermore, $\mathfrak{a} \hat{R} \cap R=\mathfrak{a}$ and $\hat{R} / \mathfrak{a} \hat{R}$ is the completion of $R / \mathfrak{a}$ with respect to the $\mathfrak{m}$-adic topology.

Theorem 1.3.6 Assume that $R$ is a Noetherian semi-local ring with Jacobson radical $\mathfrak{m}$ and let $\hat{R}$ be the completion of $R$ with respect to the $\mathfrak{m}$-adic topology. Then

1. The total quotient ring $F$ of $R$ (localization of $R$ at the set of non-zero divisors) is naturally a subring of the total quotient ring $\hat{F}$ of $\hat{R}$.

2. For any ideal $\mathfrak{a}$ of $R, \mathfrak{a} \hat{R} \cap F=\mathfrak{a}$.

In particular, $\hat{R} \cap F=R$.

\subsection{Morphisms associated with monomials and adapted morphisms}

We have the following elementary algebra result

Theorem-Definition 1.4.1 Let $R$ be an integral domain and $M$ a free $R$ module of basis $\left(e_{i}\right)_{0 \leq i \leq m}$. Let $x=r_{0} e_{0}+r_{1} e_{1}+\ldots+r_{m} e_{m}$ be a non-zero element of $M$. We set $M^{*}:=\operatorname{Hom}_{R}(M, R)$ and $M^{*}(x):=\left\{\varphi(x) \mid \varphi \in M^{*}\right\}$. Then the following assertions are equivalent:

(i) $M^{*}(x)=R$.

(ii) $\sum_{i=0}^{m} R r_{i}=R$.

(iii) There exists $\varphi \in M^{*}$ such that $\varphi(x)=1$.

(iv) There exists an $R$-submodule $N$ of $M$ such that $M=R x \oplus N$.

If $x$ satisfies the conditions above, we say that $x$ is a primitive element of $M$.

\section{Proof:}

$(i) \Leftrightarrow(i i)$ Let $\left(e_{i}^{*}\right)_{0 \leq i \leq m}$ be the basis of $M^{*}$ dual to $\left(e_{i}\right)_{0 \leq i \leq m}$. Then $M^{*}(x)$ is generated by $\left(e_{i}^{*}(x)\right)_{0 \leq i \leq m}$ and $e_{i}^{*}(x)=r_{i}$.

(ii) $\Rightarrow($ iii $)$ There exist $u_{0}, u_{1}, \ldots, u_{m} \in R$ such that $\sum_{i=0}^{m} u_{i} r_{i}=1$. If $\varphi:=$ $\sum_{i=0}^{m} u_{i} e_{i}^{*}$, then $\varphi(x)=1$. 
$($ iii $) \Rightarrow($ iv $)$ Let $N:=\operatorname{Ker} \varphi$. For all $y \in M$, we have

$$
y=\varphi(y) x+(y-\varphi(y) x) .
$$

Since $\varphi(x)=1$, we have $y \in R x+N$. Obviously, $R x \cap N=\{0\}$.

$(i v) \Rightarrow(i)$ Since $M$ is free and $R$ is an integral domain, $x$ is torsion-free (otherwise there exists $r \in R, r \neq 0$ such that $\left.\sum_{i=0}^{m}\left(r r_{i}\right) e_{i}=0\right)$. Therefore, the map

$$
R \rightarrow M, r \mapsto r x
$$

is an isomorphism of $R$-modules. Its inverse is a linear form on $R x$ which sends $x$ to 1 . Composing it with the map

$$
M \rightarrow M / N \rightarrow R
$$

we obtain a linear form $\varphi: M \rightarrow R$ such that $\varphi(x)=1$. We have $1 \in M^{*}(x)$ and thus $M^{*}(x)=R$.

We will apply the above result to the $\mathbb{Z}$-module $\mathbb{Z}^{m+1}$. Let us consider $\left(e_{i}\right)_{0 \leq i \leq m}$ the standard basis of $\mathbb{Z}^{m+1}$ and let $a:=a_{0} e_{0}+a_{1} e_{1}+\ldots+a_{m} e_{m}$ be an element of $\mathbb{Z}^{m+1}$ such that $\operatorname{gcd}\left(a_{i}\right)=1$. Then, by Bezout's theorem, there exist $u_{0}, u_{1}, \ldots, u_{m} \in \mathbb{Z}$ such that $\sum_{i=0}^{m} u_{i} a_{i}=1$ and hence $\sum_{i=0}^{m} \mathbb{Z} a_{i}=\mathbb{Z}$. By Theorem 1.4.1, there exists a $\mathbb{Z}$-submodule $N_{a}$ of $\mathbb{Z}^{m+1}$ such that $\mathbb{Z}^{m+1}=$ $\mathbb{Z} a \oplus N_{a}$. In particular, $N_{a}=\operatorname{Ker} \varphi$, where $\varphi:=\sum_{i=0}^{m} u_{i} e_{i}^{*}$. We will denote by $p_{a}: \mathbb{Z}^{m+1} \rightarrow N_{a}$ the projection of $\mathbb{Z}^{m+1}$ onto $N_{a}$ such that $\operatorname{Ker} p_{a}=\mathbb{Z} a$. We have a $\mathbb{Z}$-module isomorphism $i_{a}: N_{a} \stackrel{\sim}{\rightarrow} \mathbb{Z}^{m}$. Then $f_{a}:=i_{a} \circ p_{a}$ is a surjective $\mathbb{Z}$-module morphism $\mathbb{Z}^{m+1} \rightarrow \mathbb{Z}^{m}$ with $\operatorname{Ker} f_{a}=\mathbb{Z} a$.

Now let $R$ be an integral domain and let $x_{0}, x_{1}, \ldots, x_{m}$ be $m+1$ indeterminates over $R$. Let $G$ be the abelian group generated by all the monomials in $R\left[x_{0}^{ \pm 1}, x_{1}^{ \pm 1}, \ldots, x_{m}^{ \pm 1}\right]$ with group operation the multiplication. Then $G$ is isomorphic to the additive group $\mathbb{Z}^{m+1}$ by the isomorphism defined as follows

$$
\begin{array}{cccc}
\theta_{G}: & G & \stackrel{\sim}{\rightarrow} & \mathbb{Z}^{m+1} \\
& \prod_{i=0}^{m} x_{i}^{l_{i}} & \mapsto & \left(l_{0}, l_{1}, \ldots, l_{m}\right) .
\end{array}
$$

Lemma 1.4.2 We have $R\left[x_{0}^{ \pm 1}, x_{1}^{ \pm 1}, \ldots, x_{m}^{ \pm 1}\right]=R[G] \cong R\left[\mathbb{Z}^{m+1}\right]$.

Respectively, if $y_{1}, \ldots, y_{m}$ are $m$ indeterminates over $R$ and $H$ is the group generated by all the monomials in $R\left[y_{1}^{ \pm 1}, \ldots, y_{m}^{ \pm 1}\right]$, then $H \cong \mathbb{Z}^{m}$ and $R\left[y_{1}^{ \pm 1}, \ldots, y_{m}^{ \pm 1}\right]=R[H] \cong R\left[\mathbb{Z}^{m}\right]$ 
The morphism $F_{a}:=\theta_{H}{ }^{-1} \circ f_{a} \circ \theta_{G}: G \rightarrow H$ induces an $R$-algebra morphism

$$
\begin{array}{cccc}
\varphi_{a}: & R[G] & \rightarrow & R[H] \\
\sum_{g \in G} a_{g} g & \mapsto & \sum_{g \in G} a_{g} F_{a}(g)
\end{array}
$$

Since $F_{a}$ is surjective, the morphism $\varphi_{a}$ is also surjective. Moreover, $\operatorname{Ker} \varphi_{a}$ is generated (as an $R$-module) by the set

$$
<g-1 \mid g \in G \text { such that } \theta_{G}(g) \in \mathbb{Z} a>.
$$

From now on, let $A:=R\left[x_{0}^{ \pm 1}, x_{1}^{ \pm 1}, \ldots, x_{m}^{ \pm 1}\right]$ and $B:=R\left[y_{1}^{ \pm 1}, y_{2}^{ \pm 1}, \ldots, y_{m}^{ \pm 1}\right]$. Let $M:=\prod_{i=0}^{m} x_{i}^{a_{i}}$ and set $\varphi_{M}:=\varphi_{a}$. Then the map $\varphi_{M}$ is an $R$-algebra morphism from $A$ to $B$ with the following properties:

1. $\varphi_{M}$ is surjective.

2. $\varphi_{M}\left(x_{i}\right)$ is a monomial in $B$ for all $i=0,1, \ldots, m$.

3. $\operatorname{Ker} \varphi_{M}=(M-1) A$.

Definition 1.4.3 Let $M:=\prod_{i=0}^{m} x_{i}^{a_{i}}$ be a monomial in $A$ with $\operatorname{gcd}\left(a_{i}\right)=1$. An R-algebra morphism $\varphi_{M}: A \rightarrow B$ defined as above will be called associated with the monomial $M$.

Example 1.4.4 Let $A:=R\left[X^{ \pm 1}, Y^{ \pm 1}, Z^{ \pm 1}\right]$ and $M:=X^{5} Y^{-3} Z^{-2}$. We have $a:=(5,-3,-2)$ and

$$
(-1) \cdot 5+(-2) \cdot(-3)+0 \cdot(-2)=1 .
$$

Hence, with the notations above, we have

$$
u_{0}=-1, u_{1}=-2, u_{3}=0
$$

The map $\varphi: \mathbb{Z}^{3} \rightarrow \mathbb{Z}$ defined as

$$
\varphi:=-e_{0}^{*}-2 e_{1}^{*}
$$

has $\operatorname{Ker} \varphi=\left\{\left(x_{0}, x_{1}, x_{2}\right) \in \mathbb{Z}^{3} \mid x_{0}=-2 x_{1}\right\}=\{(-2 r, r, s) \mid r, s \in \mathbb{Z}\}=: N_{a}$.

By Theorem 1.4.1, we have $\mathbb{Z}^{3}=\mathbb{Z} a \oplus N_{a}$ and the projection $p_{a}: \mathbb{Z}^{3} \rightarrow N_{a}$ is the map

$$
\left(y_{0}, y_{1}, y_{2}\right)=: y \mapsto y-\varphi(y) a=\left(6 y_{0}+10 y_{1},-3 y_{0}-5 y_{1},-2 y_{0}-4 y_{1}+y_{2}\right) .
$$

The $\mathbb{Z}$-module $N_{a}$ is obviously isomorphic to $\mathbb{Z}^{2}$ via

$$
i_{a}:(-2 r, r, s) \mapsto(r, s) .
$$


Composing the two previous maps, we obtain a well defined surjection

$$
\begin{array}{ccc}
\mathbb{Z}^{3} & \rightarrow & \mathbb{Z}^{2} \\
\left(y_{0}, y_{1}, y_{2}\right) & \mapsto & \left(-3 y_{0}-5 y_{1},-2 y_{0}-4 y_{1}+y_{2}\right) .
\end{array}
$$

The above surjection induces (in the way described before) an $R$-algebra epimorphism

$$
\begin{array}{rlc}
\varphi_{M}: R\left[X^{ \pm 1}, Y^{ \pm 1}, Z^{ \pm 1}\right] & \rightarrow & R\left[X^{ \pm 1}, Y^{ \pm 1}\right] \\
X & \mapsto & X^{-3} Y^{-2} \\
Y & \mapsto & X^{-5} Y^{-4} \\
Z & \mapsto & Y
\end{array}
$$

By straightforward calculations, we can verify that $\operatorname{Ker} \varphi_{M}=(M-1) A$.

Proposition 1.4.5 Let $M:=\prod_{i=0}^{m} x_{i}^{a_{i}}$ be a monomial in A such that $\operatorname{gcd}\left(a_{i}\right)=$ 1. Then

1. The ideal $(M-1) A$ is a prime ideal of $A$.

2. If $\mathfrak{p}$ is a prime ideal of $R$, then the ideal $\mathfrak{q}_{M}:=\mathfrak{p} A+(M-1) A$ is also prime in $A$.

\section{Proof:}

1. Let $\varphi_{M}: A \rightarrow B$ be a morphism associated with $M$. Then $\varphi_{M}$ is surjective and $\operatorname{Ker} \varphi_{M}=(M-1) A$. By the $1^{\text {st }}$ isomorphism theorem, we have $A /(M-1) A \cong B$. Since $B$ is an integral domain, the ideal generated by $(M-1)$ is prime in $A$.

2. Set $R^{\prime}:=R / \mathfrak{p}, A^{\prime}:=R^{\prime}\left[x_{0}^{ \pm 1}, x_{1}^{ \pm 1}, \ldots, x_{m}^{ \pm 1}\right], B^{\prime}:=R^{\prime}\left[y_{1}^{ \pm 1}, \ldots, y_{m}^{ \pm 1}\right]$. The rings $R^{\prime}, A^{\prime}$ and $B^{\prime}$ are integral domains and

$$
A / \mathfrak{q}_{M} \cong A^{\prime} /(M-1) A^{\prime} \cong B^{\prime}
$$

Hence, the ideal $\mathfrak{q}_{M}$ is prime in $A$.

The following assertions are now straightforward. Nevertheless, they are stated for further reference.

Proposition 1.4.6 Let $M:=\prod_{i=0}^{m} x_{i}^{a_{i}}$ be a monomial in $A$ with $\operatorname{gcd}\left(a_{i}\right)=1$ and let $\varphi_{M}: A \rightarrow B$ be a morphism associated with $M$. Let $\mathfrak{p}$ be a prime ideal of $R$ and set $\mathfrak{q}_{M}:=\mathfrak{p} A+(M-1) A$. Then the morphism $\varphi_{M}$ has the following properties: 
1. If $f \in A$, then $\varphi_{M}(f) \in \mathfrak{p} B$ if and only if $f \in \mathfrak{q}_{M}$. Corollary 1.1.6(1) implies that

$$
A_{\mathfrak{q}_{M}} /(M-1) A_{\mathfrak{q}_{M}} \cong B_{\mathfrak{p} B}
$$

2. If $N$ is a monomial in $A$, then $\varphi_{M}(N)=1$ if and only if there exists $k \in \mathbb{Z}$ such that $N=M^{k}$.

Corollary 1.4.7 $\mathfrak{q}_{M} \cap R=\mathfrak{p}$.

Proof: $\quad$ Obviously $\mathfrak{p} \subseteq \mathfrak{q}_{M} \cap R$. Let $\xi \in R$ such that $\xi \in \mathfrak{q}_{M}$. If $\varphi_{M}$ is a morphism associated with $M$, then, by Proposition 1.4.6, $\varphi_{M}(\xi) \in \mathfrak{p} B$. But $\varphi_{M}(\xi)=\xi$ and $\mathfrak{p} B \cap R=\mathfrak{p}$. Thus $\xi \in \mathfrak{p}$.

Remark: If $m=0$ and we set $x:=x_{0}$, then $A:=R\left[x, x^{-1}\right]$ and $B:=R$. The only monomials that we can associate a morphism $A \rightarrow B$ with are $x$ and $x^{-1}$. This morphism is unique and given by $x \mapsto 1$.

The following lemma, whose proof is straightforward when arguing by contradiction, will be used in the proofs of Propositions 1.4.9 and 1.4.12.

Lemma 1.4.8 Let $G, H$ be two groups and $p: G \rightarrow H$ a group homomorphism. If $R$ is an integral domain, let us denote by $p_{R}: R[G] \rightarrow R[H]$ the $R$-algebra morphism induced by $p$. If $p_{R}$ is surjective, then $p$ is also surjective.

Proposition 1.4.9 Let $\varphi: A \rightarrow B$ be a surjective $R$-algebra morphism such that for all $i(0 \leq i \leq m), \varphi\left(x_{i}\right)$ is a monomial in $B$. Then $\varphi$ is associated with a monomial in $A$.

Proof: $\quad$ Due to the isomorphism of Lemma 1.4.2, $\varphi$ can be considered as a surjective $R$-algebra morphism

$$
\varphi: R\left[\mathbb{Z}^{m+1}\right] \rightarrow R\left[\mathbb{Z}^{m}\right]
$$

The property of $\varphi$ about the $x_{i}$ implies that the above morphism is induced by a $\mathbb{Z}$-module morphism $f: \mathbb{Z}^{m+1} \rightarrow \mathbb{Z}^{m}$, which is also surjective by Lemma 1.4.8. Since $\mathbb{Z}^{m}$ is a free $\mathbb{Z}$-module the following exact sequence sequence splits

$$
0 \rightarrow \operatorname{Ker} f \rightarrow \mathbb{Z}^{m+1} \rightarrow \mathbb{Z}^{m} \rightarrow 0
$$

and we obtain that $\mathbb{Z}^{m+1} \cong \operatorname{Ker} f \oplus \mathbb{Z}^{m}$. Therefore, $\operatorname{Ker} f$ is a $\mathbb{Z}$-module of rank 1 and there exists $a:=\left(a_{0}, a_{1}, \ldots, a_{m}\right) \in \mathbb{Z}^{m+1}$ such that $\operatorname{Ker} f=\mathbb{Z} a$. By Theorem 1.4.1, $a$ is a primitive element of $\mathbb{Z}^{m+1}$ and we must have 
$\sum_{i=0}^{m} \mathbb{Z} a_{i}=\mathbb{Z}$, whence $\operatorname{gcd}\left(a_{i}\right)=1$. By definition, the morphism $\varphi$ is associated with the monomial $\prod_{i=0}^{m} x_{i}^{a_{i}}$.

Now let $r \in\{1, \ldots, m+1\}$ and $C_{r}:=R\left[y_{r}^{ \pm 1}, \ldots, y_{m}^{ \pm 1}\right]$, where $y_{r}, \ldots, y_{m}$ are $m+1-r$ indeterminates over $R$. For $r=m+1, C_{r}=R$.

Definition 1.4.10 An R-algebra morphism $\varphi: A \rightarrow C_{r}$ is called adapted, if $\varphi=\varphi_{r} \circ \varphi_{r-1} \circ \ldots \circ \varphi_{1}$, where $\varphi_{i}$ is a morphism associated with a monomial for all $i=1, \ldots, r$. The family $\mathcal{F}:=\left\{\varphi_{r}, \varphi_{r-1}, \ldots, \varphi_{1}\right\}$ is called an adapted family for $\varphi$ whose initial morphism is $\varphi_{1}$.

Let us introduce the following notation: If $M:=\prod_{i=0}^{m} x_{i}^{c_{i}}$ is a monomial such that $\operatorname{gcd}\left(c_{i}\right)=d \in \mathbb{Z}$, then $M^{\circ}:=\prod_{i=0}^{m} x_{i}^{c_{i} / d}$.

Proposition 1.4.11 Let $\varphi: A \rightarrow C_{r}$ be an adapted morphism and $M a$ monomial in $A$ such that $\varphi(M)=1$. Then there exists an adapted family for $\varphi$ whose initial morphism is associated with $M^{\circ}$.

Proof: $\quad$ Let $M=\prod_{i=0}^{m} x_{i}^{c_{i}}$ be a monomial in $A$ such that $\varphi(M)=1$. Note that $\varphi(M)=1$ if and only if $\varphi\left(M^{\circ}\right)=1$. Therefore, we can assume that $\operatorname{gcd}\left(c_{i}\right)=1$. We will prove the desired result by induction on $r$.

If $r=1$, then, due to property 1.4.6(2), $\varphi$ must be a morphism associated with $M$. If $r=2$, set $B:=R\left[z_{1}^{ \pm 1}, \ldots, z_{m}^{ \pm 1}\right]$. Let $\varphi:=\varphi_{b} \circ \varphi_{a}$, where

- $\varphi_{a}: A \rightarrow B$ is a morphism associated with a monomial $\prod_{i=0}^{m} x_{i}^{a_{i}}$ in $A$ such that $\operatorname{gcd}\left(a_{i}\right)=1$.

- $\varphi_{b}: B \rightarrow C_{2}$ is a morphism associated with a monomial $\prod_{j=1}^{m} z_{j}^{b_{j}}$ in $B$ such that $\operatorname{gcd}\left(b_{j}\right)=1$.

By Theorem 1.4.1, the element $a:=\left(a_{0}, a_{1}, \ldots, a_{m}\right)$ is a primitive element of $\mathbb{Z}^{m+1}$ and the element $b:=\left(b_{1}, \ldots, b_{m}\right)$ is a primitive element of $\mathbb{Z}^{m}$. Therefore, there exist a $\mathbb{Z}$-submodule $N_{a}$ of $\mathbb{Z}^{m+1}$ and a $\mathbb{Z}$-submodule $N_{b}$ of $\mathbb{Z}^{m}$ such that $\mathbb{Z}^{m+1}=\mathbb{Z} a \oplus N_{a}$ and $\mathbb{Z}^{m}=\mathbb{Z} b \oplus N_{b}$. We will denote by $p_{a}: \mathbb{Z}^{m+1} \rightarrow N_{a}$ the projection of $\mathbb{Z}^{m+1}$ onto $N_{a}$ and by $p_{b}: \mathbb{Z}^{m} \rightarrow N_{b}$ the projection of $\mathbb{Z}^{m}$ onto $N_{b}$. We have isomorphisms $i_{a}: N_{a} \stackrel{\sim}{\rightarrow} \mathbb{Z}^{m}$ and $i_{b}: N_{b} \tilde{\rightarrow} \mathbb{Z}^{m-1}$.

By definition of the associated morphism, $\varphi_{a}$ is induced by the morphism $f_{a}:=i_{a} \circ p_{a}: \mathbb{Z}^{m+1} \rightarrow \mathbb{Z}^{m}$ and $\varphi_{b}$ by $f_{b}:=i_{b} \circ p_{b}: \mathbb{Z}^{m} \rightarrow \mathbb{Z}^{m-1}$. Set $f:=f_{b} \circ f_{a}$. Then $\varphi$ is the $R$-algebra morphism induced by $f$.

The morphism $f$ is surjective. Since $\mathbb{Z}^{m-1}$ is a free $\mathbb{Z}$-module, the following exact sequence sequence splits

$$
0 \rightarrow \operatorname{Ker} f \rightarrow \mathbb{Z}^{m+1} \rightarrow \mathbb{Z}^{m-1} \rightarrow 0
$$


and we obtain that $\mathbb{Z}^{m+1} \cong \operatorname{Ker} f \oplus \mathbb{Z}^{m-1}$.

Let $\tilde{b}:=i_{a}^{-1}(b)$. Then $\operatorname{Ker} f=\mathbb{Z} a \oplus \mathbb{Z} \tilde{b}$. By assumption, we have that $c:=\left(c_{0}, c_{1}, \ldots, c_{m}\right) \in \operatorname{Ker} f$. Therefore, there exist unique $\lambda_{1}, \lambda_{2} \in \mathbb{Z}$ such that $c=\lambda_{1} a+\lambda_{2} \tilde{b}$. Since $\operatorname{gcd}\left(c_{i}\right)=1$, we must also have $\operatorname{gcd}\left(\lambda_{1}, \lambda_{2}\right)=1$. Hence $\sum_{i=1}^{2} \mathbb{Z} \lambda_{i}=\mathbb{Z}$. By applying Theorem 1.4.1 to the $\mathbb{Z}$-module $\operatorname{Ker} f$, we obtain that $c$ is a primitive element of $\operatorname{Ker} f$. Consequently, $f=f^{\prime} \circ f_{c}$, where $f_{c}$ is a surjective $\mathbb{Z}$-module morphism $\mathbb{Z}^{m+1} \rightarrow \mathbb{Z}^{m}$ with $\operatorname{Ker} f_{c}=\mathbb{Z} c$ and $f^{\prime}$ is a surjective $\mathbb{Z}$-module morphism $\mathbb{Z}^{m} \rightarrow \mathbb{Z}^{m-1}$. As far as the induced $R$-algebra morphisms are concerned, we obtain that $\varphi=\varphi^{\prime} \circ \varphi_{c}$, where $\varphi_{c}$ is a morphism associated with the monomial $M=\prod_{i=0}^{m} x_{i}^{c_{i}}$ and, due to Proposition 1.4.9, $\varphi^{\prime}$ is also a morphism associated with a monomial. Thus the assertion is proven for $r=2$.

Now, let us suppose that $r>2$ and that the assertion holds for $1,2, \ldots, r-$ 1. If $\varphi=\varphi_{r} \circ \varphi_{r-1} \circ \ldots \circ \varphi_{1}$, the induction hypothesis implies that there exist morphisms associated with monomials $\varphi_{r}^{\prime}, \varphi_{r-1}^{\prime}, \ldots, \varphi_{2}^{\prime}$ such that

- $\varphi=\varphi_{r}^{\prime} \circ \ldots \circ \varphi_{2}^{\prime} \circ \varphi_{1}$.

- $\varphi_{2}^{\prime}$ is associated with the monomial $\left(\varphi_{1}(M)\right)^{\circ}$.

We have that $\varphi_{2}^{\prime}\left(\varphi_{1}(M)\right)=1$. Once more, by induction hypothesis we obtain that there exist morphisms associated with monomials $\varphi_{2}^{\prime \prime}, \varphi_{1}^{\prime \prime}$ such that

- $\varphi_{2}^{\prime} \circ \varphi_{1}=\varphi_{2}^{\prime \prime} \circ \varphi_{1}^{\prime \prime}$.

- $\varphi_{1}^{\prime \prime}$ is associated with $M^{\circ}$.

Thus we have

$$
\varphi=\varphi_{r}^{\prime} \circ \ldots \circ \varphi_{3}^{\prime} \circ \varphi_{2}^{\prime} \circ \varphi_{1}=\varphi_{r}^{\prime} \circ \ldots \circ \varphi_{3}^{\prime} \circ \varphi_{2}^{\prime \prime} \circ \varphi_{1}^{\prime \prime}
$$

and $\varphi_{1}^{\prime \prime}$ is associated with $M^{\circ}$.

Proposition 1.4.12 Let $\varphi: A \rightarrow C_{r}$ be a surjective $R$-algebra morphism such that for all $i(0 \leq i \leq m), \varphi\left(x_{i}\right)$ is a monomial in $C_{r}$. Then $\varphi$ is an adapted morphism.

Proof: We will work again by induction on $r$. For $r=1$, the above result is Proposition 1.4.9. For $r>1$, let us suppose that the result is true for $1, \ldots, r-1$. Due to the isomorphism of Lemma 1.4.2, $\varphi$ can be considered as a surjective $R$-algebra morphism

$$
\varphi: R\left[\mathbb{Z}^{m+1}\right] \rightarrow R\left[\mathbb{Z}^{m+1-r}\right] .
$$


The property of $\varphi$ about the $x_{i}$ implies that the above morphism is induced by a $\mathbb{Z}$-module morphism $f: \mathbb{Z}^{m+1} \rightarrow \mathbb{Z}^{m+1-r}$, which is also surjective by Lemma 1.4.8. Since $\mathbb{Z}^{m+1-r}$ is a free $\mathbb{Z}$-module the following exact sequence splits

$$
0 \rightarrow \operatorname{Ker} f \rightarrow \mathbb{Z}^{m+1} \rightarrow \mathbb{Z}^{m+1-r} \rightarrow 0
$$

and we obtain that $\mathbb{Z}^{m+1} \cong \operatorname{Ker} f \oplus \mathbb{Z}^{m+1-r}$. Therefore, $\operatorname{Ker} f$ is a $\mathbb{Z}$-module of rank $r$, i.e., $\operatorname{Ker} f \cong \mathbb{Z}^{r}$. We choose a primitive element $a$ of $\operatorname{Ker} f$. Then there exists a $\mathbb{Z}$-submodule $N_{a}$ of $\operatorname{Ker} f$ such that $\operatorname{Ker} f=\mathbb{Z} a \oplus N_{a}$. Since Ker $f$ is a direct summand of $\mathbb{Z}^{m+1}, a$ is also a primitive element of $\mathbb{Z}^{m+1}$ and we have

$$
\mathbb{Z}^{m+1} \cong \mathbb{Z} a \oplus N_{a} \oplus \mathbb{Z}^{m+1-r} .
$$

Thus, by Theorem 1.4.1, if $\left(a_{0}, a_{1}, \ldots, a_{m}\right)$ are the coefficients of $a$ with respect to the standard basis of $\mathbb{Z}^{m+1}$, then $\operatorname{gcd}\left(a_{i}\right)=1$.

Let us denote by $p_{a}$ the projection $\mathbb{Z}^{m+1} \rightarrow N_{a} \oplus \mathbb{Z}^{m+1-r}$ and by $p^{\prime}$ the projection $N_{a} \oplus \mathbb{Z}^{m+1-r} \rightarrow \mathbb{Z}^{m+1-r}$. We have $f=p^{\prime} \circ p_{a}$. Consequently, if we denote by $i$ the $\mathbb{Z}$-module isomorphism

$$
i: N_{a} \oplus \mathbb{Z}^{m+1-r} \stackrel{\sim}{\rightarrow} \mathbb{Z}^{m}
$$

and set $f_{a}:=i \circ p_{a}$ and $f^{\prime}:=p^{\prime} \circ i^{-1}$, we obtain that $f=f^{\prime} \circ f_{a}$. If $\varphi_{a}$ is the $R$-algebra morphism induced by $f_{a}$, then, by definition, $\varphi_{a}$ is a morphism associated with the monomial $\prod_{i=0}^{m} x_{i}^{a_{i}}$. The $R$-algebra morphism $\varphi^{\prime}$ induced by $f^{\prime}$ is a surjective morphism with the same property as $\varphi$ (it sends all monomials to monomials). By induction hypothesis, $\varphi^{\prime}$ is an adapted morphism. We have $\varphi=\varphi^{\prime} \circ \varphi_{a}$ and so $\varphi$ is also an adapted morphism.

\subsection{Irreducibility}

In this section, we will discuss the irreducibility of polynomials in a Laurent polynomial ring with coefficients in a field.

Let $k$ be a field and $y$ an indeterminate over $k$. We can use the following theorem in order to determine the irreducibility of a polynomial of the form $y^{n}-a$ in $k[y]$ (cf. [42, Chapter 6, Thm. 9.1).

Theorem 1.5.1 Let $k$ be a field, $a \in k-\{0\}$ and $n \in \mathbb{Z}$ with $n \geq 2$. The polynomial $y^{n}-a$ is irreducible in $k[y]$, if for every prime $p$ dividing $n$, we have $a \notin k^{p}$ and if 4 divides $n$, we have $a \notin-4 k^{4}$.

Let $x_{0}, x_{1}, \ldots, x_{m}$ be a set of $m+1$ indeterminates over $k$. We will apply Theorem 1.5.1 to the field $k\left(x_{1}, \ldots, x_{m}\right)$. 
Proposition 1.5.2 Let $k$ be a field. The polynomial $x_{0}^{a_{0}}-\rho \prod_{i=1}^{m} x_{i}^{a_{i}}$ with $\rho \in k-\{0\}, a_{i} \in \mathbb{Z}, \operatorname{gcd}\left(a_{i}\right)=1$ and $a_{0}>0$ is irreducible in $k\left(x_{1}, \ldots, x_{m}\right)\left[x_{0}\right]$.

Proof: If $a_{0}=1$, the polynomial is of degree 1 and thus irreducible in $k\left(x_{1}, \ldots, x_{m}\right)\left[x_{0}\right]$. If $a_{0} \geq 2$, let $p$ be a prime number dividing $a_{0}$. Let us suppose that $\rho \prod_{i=1}^{m} x_{i}^{a_{i}}$ belongs to $k\left(x_{1}, \ldots, x_{m}\right)^{p}$, i.e., that there exist $f\left(x_{1}, \ldots, x_{m}\right), g\left(x_{1}, \ldots, x_{m}\right) \in k\left[x_{1}, \ldots, x_{m}\right]$ prime to each other, with $g\left(x_{1}, \ldots, x_{m}\right) \neq 0$, such that

$$
\rho \prod_{i=1}^{m} x_{i}^{a_{i}}=\left(\frac{f\left(x_{1}, \ldots, x_{m}\right)}{g\left(x_{1}, \ldots, x_{m}\right)}\right)^{p}
$$

The above relation can be rewritten as

$$
g\left(x_{1}, \ldots, x_{m}\right)^{p} \cdot\left(\rho \prod_{\left\{i \mid a_{i} \geq 0\right\}} x_{i}^{a_{i}}\right)=f\left(x_{1}, \ldots, x_{m}\right)^{p} \cdot\left(\prod_{\left\{i \mid a_{i}<0\right\}} x_{i}^{-a_{i}}\right) .
$$

We have that

$$
\operatorname{gcd}\left(f\left(x_{1}, \ldots, x_{m}\right)^{p}, g\left(x_{1}, \ldots, x_{m}\right)^{p}\right)=1 .
$$

Since $k\left[x_{1}, \ldots, x_{m}\right]$ is a unique factorization domain and the $x_{i}$ are irreducible in $k\left[x_{1}, \ldots, x_{m}\right]$, we also have that

$$
\operatorname{gcd}\left(\prod_{\left\{i \mid a_{i} \geq 0\right\}} x_{i}^{a_{i}}, \prod_{\left\{i \mid a_{i}<0\right\}} x_{i}^{-a_{i}}\right)=1 .
$$

As a result,

$$
f\left(x_{1}, \ldots, x_{m}\right)^{p}=\lambda \rho \cdot \prod_{\left\{i \mid a_{i} \geq 0\right\}} x_{i}^{a_{i}} \text { and } g\left(x_{1}, \ldots, x_{m}\right)^{p}=\lambda \cdot \prod_{\left\{i \mid a_{i}<0\right\}} x_{i}^{-a_{i}} .
$$

for some $\lambda \in k-\{0\}$. If $(\lambda \rho)^{1 / p}, \lambda^{1 / p} \notin k$, we have arrived to a contradiction. Suppose that $(\lambda \rho)^{1 / p}, \lambda^{1 / p} \in k$. Once more, the fact that $k\left[x_{1}, \ldots, x_{m}\right]$ is a unique factorization domain and the $x_{i}$ are irreducible in $k\left[x_{1}, \ldots, x_{m}\right]$ implies that

$$
f\left(x_{1}, \ldots, x_{m}\right)=(\lambda \rho)^{1 / p} \cdot \prod_{\left\{i \mid a_{i} \geq 0\right\}} x_{i}^{b_{i}} \text { and } g\left(x_{1}, \ldots, x_{m}\right)=\lambda^{1 / p} \cdot \prod_{\left\{i \mid a_{i}<0\right\}} x_{i}^{-b_{i}},
$$

with $b_{i} \in \mathbb{Z}$ and $b_{i} p=a_{i}, \forall i=1, \ldots, m$. Since $p$ divides $a_{0}$, this contradicts the fact that $\operatorname{gcd}\left(a_{i}\right)=1$. In the same way, we can show that if 4 divides $a_{0}$, then $\rho \prod_{i=1}^{m} x_{i}^{a_{i}} \notin-4 k\left(x_{1}, \ldots, x_{m}\right)^{4}$. Thus, by Theorem 1.5.1, $x_{0}^{a_{0}}-\rho \prod_{i=1}^{m} x_{i}^{a_{i}}$ is irreducible in $k\left(x_{1}, \ldots, x_{m}\right)\left[x_{0}\right]$.

Thanks to Lemma 1.5.4, the above proposition implies that 
Proposition 1.5.3 Let $k$ be a field. The polynomial $x_{0}^{a_{0}}-\rho \prod_{i=1}^{m} x_{i}^{a_{i}}$ with $\rho \in$ $k-\{0\}, a_{i} \in \mathbb{Z}, \operatorname{gcd}\left(a_{i}\right)=1$ and $a_{0}>0$ is irreducible in $k\left[x_{1}^{ \pm 1}, \ldots, x_{m}^{ \pm 1}\right]\left[x_{0}\right]$.

Lemma 1.5.4 Let $R$ be an integral domain with field of fractions $F$ and $f(x)$ a polynomial in $R[x]$. If $f(x)$ is irreducible in $F[x]$ and at least one of its coefficients is a unit in $R$, then $f(x)$ is irreducible in $R[x]$.

Proof: If $f(x)=g(x) h(x)$ for two polynomials $g(x), h(x) \in R[x]$, then $g(x) \in R$ or $h(x) \in R$. Let us suppose that $g(x) \in R$. Since one of the coefficients of $f(x)$ is a unit in $R, g(x)$ must also be a unit in $R$. Thus, $f(x)$ is irreducible in $R[x]$.

The next result is an immediate consequence of Proposition 1.5.3 and will be used in the proof of Theorem 1.5.6.

Corollary 1.5.5 Let $M:=\prod_{i=0}^{m} x_{i}^{a_{i}}$ be a monomial in $k\left[x_{0}^{ \pm 1}, x_{1}^{ \pm 1}, \ldots, x_{m}^{ \pm 1}\right]$ such that $\operatorname{gcd}\left(a_{i}\right)=1$ and let $\rho \in k-\{0\}$. Then $M-\rho$ is an irreducible element of $k\left[x_{0}^{ \pm 1}, x_{1}^{ \pm 1}, \ldots, x_{m}^{ \pm 1}\right]$.

Theorem 1.5.6 Let $M:=\prod_{i=0}^{m} x_{i}^{a_{i}}$ be a monomial in $k\left[x_{0}^{ \pm 1}, x_{1}^{ \pm 1}, \ldots, x_{m}^{ \pm 1}\right]$ such that $\operatorname{gcd}\left(a_{i}\right)=1$. If $f(x)$ is an irreducible element of $k[x]$ such that $f(0) \neq 0$, then $f(M)$ is irreducible in $k\left[x_{0}^{ \pm 1}, x_{1}^{ \pm 1}, \ldots, x_{m}^{ \pm 1}\right]$.

Proof: $\quad$ Suppose that $f(M)=g \cdot h$ with $g, h \in k\left[x_{0}^{ \pm 1}, x_{1}^{ \pm 1}, \ldots, x_{m}^{ \pm 1}\right]$. Let $\rho_{1}, \ldots, \rho_{n}$ be the roots of $f(x)$ in a splitting field $k^{\prime}$. Then

$$
f(x)=a\left(x-\rho_{1}\right) \ldots\left(x-\rho_{n}\right)
$$

for some $a \in k-\{0\}$, whence

$$
f(M)=a\left(M-\rho_{1}\right) \ldots\left(M-\rho_{n}\right) .
$$

By Corollary [1.5.5, $M-\rho_{j}$ is irreducible in $k^{\prime}\left[x_{0}^{ \pm 1}, x_{1}^{ \pm 1}, \ldots, x_{m}^{ \pm 1}\right]$ for all $j \in$ $\{1, \ldots, n\}$. Since $k^{\prime}\left[x_{0}^{ \pm 1}, x_{1}^{ \pm 1}, \ldots, x_{m}^{ \pm 1}\right]$ is a unique factorization domain, we must have

$$
g=r \cdot\left(\prod_{i=0}^{m} x_{i}^{b_{i}}\right) \cdot\left(M-\rho_{j_{1}}\right) \ldots\left(M-\rho_{j_{s}}\right)
$$

for some $r \in k-\{0\}, b_{i} \in \mathbb{Z}$ and $j_{1}, \ldots, j_{s} \in\{1, \ldots, n\}$ with $j_{1}<j_{2}<\ldots<$ $j_{s}$. Thus, there exists $g^{\prime}(x) \in k[x]$ such that

$$
g=\left(\prod_{i=0}^{m} x_{i}^{b_{i}}\right) \cdot g^{\prime}(M)
$$


Respectively, there exists $h^{\prime}(x) \in k[x]$ such that

$$
h=\left(\prod_{i=0}^{m} x_{i}^{-b_{i}}\right) \cdot h^{\prime}(M) .
$$

Thus, we obtain that

$$
f(M)=g^{\prime}(M) h^{\prime}(M) .
$$

Since $\operatorname{gcd}\left(a_{i}\right)=1$, there exist integers $\left(u_{i}\right)_{0 \leq i \leq m}$ such that $\sum_{i=0}^{m} u_{i} a_{i}=1$. Let us now consider the $k$-algebra specialization

$$
\begin{aligned}
\varphi: k\left[x_{0}^{ \pm 1}, x_{1}^{ \pm 1}, \ldots, x_{m}^{ \pm 1}\right] & \rightarrow k[x] \\
x_{i} & \mapsto x^{u_{i}} .
\end{aligned}
$$

Then $\varphi(M)=\varphi\left(\prod_{i=0}^{m} x_{i}^{a_{i}}\right)=x^{\sum_{i=0}^{m} u_{i} a_{i}}=x$. If we apply $\varphi$ to the equality $f(M)=g^{\prime}(M) h^{\prime}(M)$, we obtain that

$$
f(x)=g^{\prime}(x) h^{\prime}(x) .
$$

Since $f(x)$ is irreducible in $k[x]$, we must have that either $g^{\prime}(x) \in k$ or $h^{\prime}(x) \in$ $k$. Respectively, we deduce that either $g$ or $h$ is a unit in $k\left[x_{0}^{ \pm 1}, x_{1}^{ \pm 1}, \ldots, x_{m}^{ \pm 1}\right]$. 


\section{Chapter 2}

\section{On Blocks}

The second chapter focuses on the study of blocks of algebras of finite type and in particular, of symmetric algebras. In the first section, we introduce the notion of blocks and show how we can reduce, in many different ways, the problem of the determination of the blocks of an algebra to easier cases (mostly by changing the ring of definition). In the second section, we give the definition of a symmetric algebra and introduce the Schur elements associated to its irreducible characters. We explain why the Schur elements play an essential role in the determination of the blocks of a symmetric algebra. Now, if we know the blocks of a symmetric algebra, then, given that certain conditions are satisfied, we can obtain the Schur elements and the blocks of its subalgebras with the use of a generalization of some classical results, known as "Clifford theory", to the case of "twisted symmetric algebras of finite groups". This is the object of section 2.3. Finally, in the last section, we present some more results on the block theory of symmetric algebras, which are related to the representation theory of these algebras. We define decomposition maps and generalize results of Brauer theory to the case of symmetric algebras defined over discrete valuation rings. Moreover, in subsection 2.4.4, we give a new criterion for a symmetric algebra to be split semisimple.

Throughout this chapter, we use the simple example of group algebras in order to illustrate some of the notions. However, when we introduce Hecke algebras in Chapter 4, we will see that they are symmetric too. In particular, the Appendix contains numerous applications of Clifford theory to Hecke algebras. If the reader feels that they are in need of more examples than the ones given in Chapter 2, they should feel free to refer to Chapter 4 or the Appendix. 


\section{$2.1 \quad$ Generalities}

Let $\mathcal{O}$ be a commutative ring with a unit element and $A$ be an $\mathcal{O}$-algebra. We denote by $Z A$ the center of $A$.

An idempotent in $A$ is an element $e$ such that $e^{2}=e$. We say that $e$ is a central idempotent, if it is an idempotent in $Z A$. Two idempotents $e_{1}, e_{2}$ are orthogonal, if $e_{1} e_{2}=e_{2} e_{1}=0$. Finally, an idempotent $e$ is primitive, if $e \neq 0$ and $e$ can not be expressed as the sum of two non-zero orthogonal idempotents.

Definition 2.1.1 The block-idempotents of $A$ are the central primitive idempotents of $A$.

Let $e$ be a block-idempotent of $A$. The two sided ideal $A e$ inherits a structure of algebra, where the composition laws are those of $A$ and the unit element is $e$. The application

$$
\begin{aligned}
\pi_{e}: A & \rightarrow A e \\
h & \mapsto h e
\end{aligned}
$$

is an epimorphism of algebras. The algebra $A e$ is called a block of $A$. From now on, abusing the language, we will also call blocks the block-idempotents of $A$.

Lemma 2.1.2 The blocks of $A$ are mutually orthogonal.

Proof: $\quad$ Let $e$ be a block and $f$ a central idempotent of $A$ with $f \neq e$. Then $e f$ and $e-e f$ are also central idempotents. We have $e=e f+(e-e f)$ and due to the primitivity of $e$, we deduce that either $e f=0$ or $e=e f$. If $f$ is a block too, then either $e f=0$ or $f=e f=e$. Therefore, $f$ is orthogonal to $e$.

The above lemma gives rise to the following proposition.

Proposition 2.1.3 Suppose that the unity element 1 of $A$ can be expressed as a sum of blocks: $1=\sum_{e \in E}$ e. Then

1. The set $E$ is the set of all the blocks of $A$.

2. The family of morphisms $\left(\pi_{e}\right)_{e \in E}$ defines an isomorphism of algebras

$$
A \stackrel{\sim}{\rightarrow} \prod_{e \in E} A e .
$$


Proof: If $f$ is a block, then $f=\sum_{e \in E} e f$. Due to lemma 2.1.2, there exists $e \in E$ such that $f=e$.

In the above context ( 1 is a sum of blocks), let us denote by $\operatorname{Bl}(A)$ the set of all the blocks of $A$. Proposition 2.1.3 implies that the category ${ }_{A} \mathbf{m o d}$ of $A$-modules is a direct sum of the categories associated with the blocks:

$$
{ }_{A} \bmod \stackrel{\sim}{\rightarrow} \bigoplus_{e \in \operatorname{Bl}(A)} A e \bmod .
$$

In particular, every representation of the $\mathcal{O}$-algebra $A e$ defines (by composition with $\pi_{e}$ ) a representation of $A$ and we say, abusing the language, that it "belongs to the block $e$ ".

Every indecomposable representation of $A$ belongs to one and only one block. Thus the following partitions are defined:

$$
\operatorname{Ind}(A)=\bigsqcup_{e \in \operatorname{Bl}(A)} \operatorname{Ind}(A, e) \text { and } \operatorname{Irr}(A)=\bigsqcup_{e \in \operatorname{Bl}(A)} \operatorname{Irr}(A, e)
$$

where $\operatorname{Ind}(A)$ (resp. $\operatorname{Irr}(A))$ denotes the set of indecomposable (resp. irreducible) representations of $A$ and $\operatorname{Ind}(A, e)(\operatorname{resp} . \operatorname{Irr}(A, e))$ denotes the set of the elements of $\operatorname{Ind}(A)$ (resp. $\operatorname{Irr}(A))$ which belong to $e$.

We will consider two situations where 1 is a sum of blocks.

First case: Suppose that 1 is a sum of orthogonal primitive idempotents, i.e., $1=\sum_{i \in P} i$, where

- every $i \in P$ is a primitive idempotent,

- if $i, j \in P, i \neq j$, then $i j=j i=0$.

Let us consider the equivalence relation $\mathcal{B}$ defined on $P$ as the symmetric and transitive closure of the relation " $i A j \neq\{0\}$ ". Thus $(i \mathcal{B} j)$ if and only if there exist $i_{0}, i_{1}, \ldots, i_{n} \in P$ with $i_{0}=i$ and $i_{n}=j$ such that for all $k \in\{1, \ldots, n\}$, $i_{k-1} A i_{k} \neq\{0\}$ or $i_{k} A i_{k-1} \neq\{0\}$. To every equivalence class $B$ of $P$ with respect to $\mathcal{B}$, we associate the idempotent $e_{B}:=\sum_{i \in B} i$.

Proposition 2.1.4 The map $B \mapsto e_{B}$ is a bijection between the set of equivalence classes of $\mathcal{B}$ and the set of blocks of $A$. In particular, we have that $1=\sum_{B \in P / \mathcal{B}} e_{B}$ and 1 is sum of the blocks of $A$. 
Proof: $\quad$ It is clear that $1=\sum_{B \in P / \mathcal{B}} e_{B}$. Let $a \in A$ and let $B, B^{\prime}$ be two equivalence classes of $\mathcal{B}$ with $B \neq B^{\prime}$. Then, by definition of the relation $\mathcal{B}$, $e_{B} a e_{B^{\prime}}=0$. Since $1=\sum_{B \in P / \mathcal{B}} e_{B}$, we have that $e_{B} a=e_{B} a e_{B}=a e_{B}$. Thus $e_{B} \in Z A$ for all $B \in P / \mathcal{B}$.

It remains to show that for all $B \in P / \mathcal{B}$, the central idempotent $e_{B}$ is primitive. Suppose that $e_{B}=e+f$, where $e$ and $f$ are two orthogonal primitive idempotents in $Z A$. Then we have a partition $B=B_{e} \sqcup B_{f}$, where $B_{e}:=\{i \in B \mid i e=i\}$ and $B_{f}:=\{j \in B \mid j f=j\}$. For all $i \in B_{e}$ and $j \in B_{f}$, we have $i A j=i e A f j=i A e f j=\{0\}$ and so no element of $B_{e}$ can be $\mathcal{B}$-equivalent to an element of $B_{f}$. Therefore, we must have either $B_{e}=\emptyset$ or $B_{f}=\emptyset$, which implies that either $e=0$ or $f=0$.

Second case: Suppose that $Z A$ is a subalgebra of a commutative algebra $C$ where 1 is a sum of blocks. For example, if $A$ is of finite type over $\mathcal{O}$, where $\mathcal{O}$ is an integral domain with field of fractions $F$, we can choose $C$ to be the center of the algebra $F A:=F \otimes_{\mathcal{O}} A$. We set $1=\sum_{e \in E} e$, where $E$ is the set of blocks of $C$. For all $S \subseteq E$, set $e_{S}:=\sum_{e \in S} e$. A subset $S$ of $E$ is "on $Z A$ " if $e_{S} \in Z A$. If $S$ and $T$ are on $Z A$, then $S \cap T$ is on $Z A$.

Proposition 2.1.5 Let us denote by $\mathcal{P}_{E}(Z A)$ the set of non-empty subsets $B$ of $E$ which are on $Z A$ and are minimal for these two properties. Then the map $\mathcal{P}_{E}(Z A) \rightarrow A, B \mapsto e_{B}$ induces a bijection between $\mathcal{P}_{E}(Z A)$ and the set of blocks of $A$. We have $1=\sum_{B \in \mathcal{P}_{E}(Z A)} e_{B}$.

Proof: $\quad$ Since every idempotent in $C$ is of the form $e_{S}$ for some $S \subseteq E$, it is clear that $e_{B}$ is a central primitive idempotent of $A$, for all $B \in \mathcal{P}_{E}(Z A)$. It remains to show that

1. If $B, B^{\prime}$ are two distinct elements of $\mathcal{P}_{E}(Z A)$, then $B \cap B^{\prime}=\emptyset$.

2. $\mathcal{P}_{E}(Z A)$ is a partition of $E$.

These two properties, stated in terms of idempotents, mean:

1. If $B, B^{\prime}$ are two distinct elements of $\mathcal{P}_{E}(Z A)$, then $e_{B}$ and $e_{B^{\prime}}$ are orthogonal.

2. $1=\sum_{B \in \mathcal{P}_{E}(Z A)} e_{B}$.

Let us prove them:

1. We have $e_{B} e_{B^{\prime}}=e_{B \cap B^{\prime}}$ and so $B \cap B^{\prime}=\emptyset$, because $B$ and $B^{\prime}$ are minimal. 
2. Set $F:=\bigcup_{B \in \mathcal{P}_{E}(Z A)} B$. Then $e_{F}=\sum_{B \in \mathcal{P}_{E}(Z A)} e_{B} \in Z A$. Then $1-e_{F}=e_{E-F} \in Z A$, which means that $E-F$ is on $Z A$. If $E-F \neq \emptyset$, then $E-F$ contains an element of $\mathcal{P}_{E}(Z A)$ in contradiction to the definition of $F$. Thus $F=E$ and $\mathcal{P}_{E}(Z A)$ is a partition of $E$.

Now let us assume that

- $\mathcal{O}$ is a commutative integral domain with field of fractions $F$,

- $K$ is a field extension of $F$,

- $A$ is an $\mathcal{O}$-algebra, free and finitely generated as an $\mathcal{O}$-module.

Suppose that the $K$-algebra $K A:=K \otimes_{\mathcal{O}} A$ is semisimple. Then $K A$ is isomorphic, by assumption, to a direct product of simple algebras:

$$
K A \cong \prod_{\chi \in \operatorname{Irr}(K A)} M_{\chi}
$$

where $\operatorname{Irr}(K A)$ denotes the set of irreducible characters of $K A$ and $M_{\chi}$ is a simple $K$-algebra.

For all $\chi \in \operatorname{Irr}(K A)$, we denote by $\pi_{\chi}: K A \rightarrow M_{\chi}$ the projection onto the $\chi$-factor and by $e_{\chi}$ the element of $K A$ such that

$$
\pi_{\chi^{\prime}}\left(e_{\chi}\right)= \begin{cases}1_{M_{\chi}}, & \text { if } \chi=\chi^{\prime} \\ 0, & \text { if } \chi \neq \chi^{\prime}\end{cases}
$$

The following theorem results directly from Propositions 2.1.3 and 2.1.5.

\section{Theorem 2.1.6}

1. We have $1=\sum_{\chi \in \operatorname{Irr}(K A)} e_{\chi}$ and the set $\left\{e_{\chi}\right\}_{\chi \in \operatorname{Irr}(K A)}$ is the set of all the blocks of the algebra $K A$.

2. There exists a unique partition $\operatorname{Bl}(A)$ of $\operatorname{Irr}(K A)$ such that

(a) For all $B \in \mathrm{Bl}(A)$, the idempotent $e_{B}:=\sum_{\chi \in B} e_{\chi}$ is a block of $A$.

(b) We have $1=\sum_{B \in \operatorname{Bl}(A)} e_{B}$ and for every central idempotent e of $A$, there exists a subset $\mathrm{Bl}(A, e)$ of $\mathrm{Bl}(A)$ such that

$$
e=\sum_{B \in \operatorname{Bl}(A, e)} e_{B}
$$

In particular the set $\left\{e_{B}\right\}_{B \in \mathrm{Bl}(A)}$ is the set of all the blocks of $A$. 
Remarks:

- If $\chi \in B$ for some $B \in \operatorname{Bl}(A)$, we say that " $\chi$ belongs to the block $e_{B}$ ".

- For all $B \in \operatorname{Bl}(A)$, we have

$$
K A e_{B} \cong \prod_{\chi \in B} M_{\chi}
$$

From now on, we make the following assumptions

\section{Assumptions 2.1.7}

(int) The ring $\mathcal{O}$ is a Noetherian and integrally closed domain with field of fractions $F$ and $A$ is an $\mathcal{O}$-algebra which is free and finitely generated as an $\mathcal{O}$-module.

(spl) The field $K$ is a finite Galois extension of $F$ and the algebra $K A$ is split (i.e., for every simple $K A$-module $V$, $\operatorname{End}_{K A}(V) \cong K$ ) semisimple.

We denote by $\mathcal{O}_{K}$ the integral closure of $\mathcal{O}$ in $K$.

Example 2.1.8 Let $C_{6}$ be the cyclic group of order 6 and $A:=\mathbb{Z}\left[C_{6}\right]$. If we take $K:=\mathbb{Q}\left(\zeta_{6}\right)$, where $\zeta_{6}:=\exp \left(\frac{2 \pi i}{6}\right)$, then the assumptions 2.1.7 are satisfied.

\subsubsection{Blocks and integral closure}

The Galois group $\operatorname{Gal}(K / F)$ acts on $K A=K \otimes_{\mathcal{O}} A$ (viewed as an $F$-algebra) as follows: if $\sigma \in \operatorname{Gal}(K / F)$ and $\lambda \otimes a \in K A$, then $\sigma(\lambda \otimes a):=\sigma(\lambda) \otimes a$.

If $V$ is a $K$-vector space and $\sigma \in \operatorname{Gal}(K / F)$, we denote by ${ }^{\sigma} V$ the $K$ vector space defined on the additive group $V$ with multiplication $\lambda . v:=$ $\sigma^{-1}(\lambda) v$ for all $\lambda \in K$ and $v \in V$. If $\rho: K A \rightarrow \operatorname{End}_{K}(V)$ is a representation of the $K$-algebra $K A$, then its composition with the action of $\sigma^{-1}$ is also a representation ${ }^{\sigma} \rho: K A \rightarrow \operatorname{End}_{K}\left({ }^{\sigma} V\right):$

$$
K A \stackrel{\sigma^{-1}}{\longrightarrow} K A \stackrel{\rho}{\longrightarrow} \operatorname{End}_{K}(V) .
$$

We denote by ${ }^{\sigma} \chi$ the character of ${ }^{\sigma} \rho$ and we define the action of $\operatorname{Gal}(K / F)$ on $\operatorname{Irr}(K A)$ as follows: if $\sigma \in \operatorname{Gal}(K / F)$ and $\chi \in \operatorname{Irr}(K A)$, then

$$
\sigma(\chi):={ }^{\sigma} \chi=\sigma \circ \chi \circ \sigma^{-1} \text {. }
$$

This operation induces an action of $\operatorname{Gal}(K / F)$ on the set of blocks of $K A$ :

$$
\sigma\left(e_{\chi}\right)=e_{\sigma} \text { for all } \sigma \in \operatorname{Gal}(K / F), \chi \in \operatorname{Irr}(K A) .
$$


Hence, the group $\operatorname{Gal}(K / F)$ acts on the set of idempotents of $Z \mathcal{O}_{K} A$ and thus on the set of blocks of $\mathcal{O}_{K} A$. Since $F \cap \mathcal{O}_{K}=\mathcal{O}$, the idempotents of $Z A$ are the idempotents of $Z \mathcal{O}_{K} A$ which are fixed by the action of $\operatorname{Gal}(K / F)$. As a consequence, the primitive idempotents of $Z A$ are sums of the elements of the orbits of $\operatorname{Gal}(K / F)$ on the set of primitive idempotents of $Z \mathcal{O}_{K} A$. Thus, the blocks of $A$ are in bijection with the orbits of $\operatorname{Gal}(K / F)$ on the set of blocks of $\mathcal{O}_{K} A$. The following proposition is just a reformulation of this result.

\section{Proposition 2.1.9}

1. Let $B$ be a block of $A$ and $B^{\prime}$ a block of $\mathcal{O}_{K} A$ contained in $B$. If $\operatorname{Gal}(K / F)_{B^{\prime}}$ denotes the stabilizer of $B^{\prime}$ in $\operatorname{Gal}(K / F)$, then

$$
B=\bigcup_{\sigma \in \operatorname{Gal}(K / F) / \operatorname{Gal}(K / F)_{B^{\prime}}} \sigma\left(B^{\prime}\right) \text { i.e., } \quad e_{B}=\sum_{\sigma \in \operatorname{Gal}(K / F) / \operatorname{Gal}(K / F)_{B^{\prime}}} \sigma\left(e_{B^{\prime}}\right) .
$$

2. Two characters $\chi, \psi \in \operatorname{Irr}(K A)$ are in the same block of $A$ if and only if there exists $\sigma \in \operatorname{Gal}(K / F)$ such that $\sigma(\chi)$ and $\psi$ belong to the same block of $\mathcal{O}_{K} A$.

Remark: For all $\chi \in B^{\prime}$, we have $\operatorname{Gal}(K / F)_{\chi} \subseteq \operatorname{Gal}(K / F)_{B^{\prime}}$.

The assertion (2) of the proposition above allows us to transfer the problem of the classification of the blocks of $A$ to that of the classification of the blocks of $\mathcal{O}_{K} A$.

\subsubsection{Blocks and prime ideals}

We denote by $\operatorname{Spec}_{1}(\mathcal{O})$ the set of prime ideals of height 1 of $\mathcal{O}$. Since $\mathcal{O}$ is Noetherian and integrally closed, Proposition 1.2.26 implies that $\mathcal{O}$ is a Krull ring. By Theorem 1.2.24, we have

$$
\mathcal{O}=\bigcap_{\mathfrak{p} \in \operatorname{Spec}_{1}(\mathcal{O})} \mathcal{O}_{\mathfrak{p}}
$$

where $\mathcal{O}_{\mathfrak{p}}:=\{x \in F \mid(\exists a \in \mathcal{O}-\mathfrak{p})(a x \in \mathcal{O})\}$ is the localization of $\mathcal{O}$ at $\mathfrak{p}$. In particular, $\mathcal{O}_{\mathfrak{p}}$ is a discrete valuation ring.

Let $\mathfrak{p}$ be a prime ideal of $\mathcal{O}$ and $\mathcal{O}_{\mathfrak{p}} A:=\mathcal{O}_{\mathfrak{p}} \otimes_{\mathcal{O}} A$. The blocks of $\mathcal{O}_{\mathfrak{p}} A$ are called the "p-blocks of $A$ ". If $\chi, \psi \in \operatorname{Irr}(K A)$ belong to the same block of $\mathcal{O}_{\mathfrak{p}} A$, we write $\chi \sim_{\mathfrak{p}} \psi$. 
Proposition 2.1.10 Two characters $\chi, \psi \in \operatorname{Irr}(K A)$ belong to the same block of $A$ if and only if there exist a finite sequence $\chi_{0}, \chi_{1}, \ldots, \chi_{n} \in \operatorname{Irr}(K A)$ and a finite sequence $\mathfrak{p}_{1}, \ldots, \mathfrak{p}_{n} \in \operatorname{Spec}_{1}(\mathcal{O})$ such that

- $\chi_{0}=\chi$ and $\chi_{n}=\psi$,

- for all $j(1 \leq j \leq n), \chi_{j-1} \sim_{\mathfrak{p}_{j}} \chi_{j}$.

Proof: Let us denote by $\sim$ the equivalence relation on $\operatorname{Irr}(K A)$ defined as the transitive closure of the relation "there exists $\mathfrak{p} \in \operatorname{Spec}_{1}(\mathcal{O})$ such that $\chi \sim_{\mathfrak{p}} \psi "$. Thus, we have to show that $\chi \sim \psi$ if and only if $\chi$ and $\psi$ belong to the same block of $A$.

We will first show that the equivalence relation $\sim$ is finer than the relation "being in the same block of $\mathrm{A}$ ". Let $B$ be a block of $A$. Then $B$ is a subset of $\operatorname{Irr}(K A)$ such that $\sum_{\chi \in B} e_{\chi} \in A$. Since $\mathcal{O}=\bigcap_{\mathfrak{p} \in \operatorname{Spec}_{1}(\mathcal{O})} \mathcal{O}_{\mathfrak{p}}$, we have that $\sum_{\chi \in B} e_{\chi} \in \mathcal{O}_{\mathfrak{p}} A$ for all $\mathfrak{p} \in \operatorname{Spec}_{1}(\mathcal{O})$. Therefore, by Theorem 2.1.6, $C$ is a union of blocks of $\mathcal{O}_{\mathfrak{p}} A$ for all $\mathfrak{p} \in \operatorname{Spec}_{1}(\mathcal{O})$ and, hence, a union of equivalence classes of $\sim$.

Now we will show that the relation "being in the same block of A" is finer than the relation $\sim$. Let $C$ be an equivalence class of $\sim$. Then $\sum_{\chi \in C} e_{\chi} \in \mathcal{O}_{\mathfrak{p}} A$ for all $\mathfrak{p} \in \operatorname{Spec}_{1}(\mathcal{O})$. Thus $\sum_{\chi \in C} e_{\chi} \in \bigcap_{\mathfrak{p} \in \operatorname{Spec}_{1}(\mathcal{O})} \mathcal{O}_{\mathfrak{p}} A=A$ and $C$ is a union of blocks of $A$.

Remark: More generally, if we denote by $\operatorname{Spec}(\mathcal{O})$ the set of prime ideals of $\mathcal{O}$, then

$$
\mathcal{O}=\bigcap_{\mathfrak{p} \in \operatorname{Spec}(\mathcal{O})} \mathcal{O}_{\mathfrak{p}}
$$

Hence, Proposition 2.1.10 is also true, if we replace $\operatorname{Spec}_{1}(\mathcal{O})$ by $\operatorname{Spec}(\mathcal{O})$. However, in this case, $\mathcal{O}_{\mathfrak{p}}$ is not always a discrete valuation ring.

Thanks to Proposition 2.1.10, we can transfer the problem of the determination of the blocks of $A$ to that of the determination of the $\mathfrak{p}$-blocks of $A$, where $\mathfrak{p}$ runs over the set or prime ideals (of height 1 ) of $\mathcal{O}$.

Example 2.1.11 Let $C_{6}$ be the cyclic group of order 6 and $A:=\mathbb{Z}\left[C_{6}\right]$. The group $C_{6}$ (and thus the algebra $\mathbb{Q}\left(\zeta_{6}\right)\left[C_{6}\right]$ ) has 6 irreducible characters $\chi_{1}, \ldots, \chi_{6}$, where

- $\chi_{1}$ is the trivial character,

- $\chi_{4}(g) \in \mathbb{R}$ for all $g \in C_{6}$,

- $\chi_{2}$ is the conjugate of $\chi_{6}$, 
- $\chi_{3}$ is the conjugate of $\chi_{5}$,

- $\chi_{2}(g) \chi_{3}(g)=\chi_{4}(g)$ for all $g \in C_{6}$.

If $p$ is a prime number, then the localization of $\mathbb{Z}$ at $p \mathbb{Z}$ is a discrete valuation ring. By Brauer theory, the $p$-blocks of $C_{6}$ are trivial, unless $p$ divides the order of $C_{6}$. The 2-blocks of $C_{6}$ are $\left\{\chi_{1}, \chi_{4}\right\},\left\{\chi_{2}, \chi_{5}\right\}$ and $\left\{\chi_{3}, \chi_{6}\right\}$, whereas the 3-blocks of $C_{6}$ are $\left\{\chi_{1}, \chi_{3}, \chi_{5}\right\}$ and $\left\{\chi_{2}, \chi_{4}, \chi_{6}\right\}$. Proposition 2.1.10 implies that all the irreducible characters of $C_{6}$ belong to the same block of $A$.

\subsubsection{Blocks and quotient blocks}

Let $\mathfrak{p}$ be a prime ideal of $\mathcal{O}$. Then $\mathcal{O}_{\mathfrak{p}}$ is a local ring, whose maximal ideal is $\mathfrak{p}^{\prime}:=\mathfrak{p} \mathcal{O}_{\mathfrak{p}}$. Let $\mathfrak{q}$ be a prime ideal of $\mathcal{O}$ such that $\mathfrak{q} \subseteq \mathfrak{p}$. Then $\mathfrak{q}^{\prime}:=\mathfrak{q} \mathcal{O}_{\mathfrak{p}}$ is a prime ideal of $\mathcal{O}_{\mathfrak{p}}$. The natural surjection $\pi_{\mathfrak{q}}: \mathcal{O}_{\mathfrak{p}} \rightarrow \mathcal{O}_{\mathfrak{p}} / \mathfrak{q}^{\prime}$ extends to a morphism $\pi_{\mathfrak{q}}: \mathcal{O}_{\mathfrak{p}} A \rightarrow\left(\mathcal{O}_{\mathfrak{p}} / \mathfrak{q}^{\prime}\right) A$, which in turn induces a morphism

$$
\pi_{\mathfrak{q}}: Z \mathcal{O}_{\mathfrak{p}} A \rightarrow Z\left(\mathcal{O}_{\mathfrak{p}} / \mathfrak{q}^{\prime}\right) A
$$

The following lemma will serve for the proof of Proposition 2.1.13.

Lemma 2.1.12 Let $e$ be an idempotent of $\mathcal{O}_{\mathfrak{p}} A$ whose image $\bar{e}$ in $\left(\mathcal{O}_{\mathfrak{p}} / \mathfrak{q}^{\prime}\right) A$ is central. Then e is central.

Proof: We have the following equality:

$$
\mathcal{O}_{\mathfrak{p}} A=e \mathcal{O}_{\mathfrak{p}} A e \oplus e \mathcal{O}_{\mathfrak{p}} A(1-e) \oplus(1-e) \mathcal{O}_{\mathfrak{p}} A e \oplus(1-e) \mathcal{O}_{\mathfrak{p}} A(1-e) .
$$

Since $\bar{e}$ is central, we have

$$
\bar{e}\left(\mathcal{O}_{\mathfrak{p}} / \mathfrak{q}^{\prime}\right) A(1-\bar{e})=(1-\bar{e})\left(\mathcal{O}_{\mathfrak{p}} / \mathfrak{q}^{\prime}\right) A \bar{e}=\{0\},
$$

i.e.,

$$
e \mathcal{O}_{\mathfrak{p}} A(1-e) \subseteq \mathfrak{q} \mathcal{O}_{\mathfrak{p}} A \text { and }(1-e) \mathcal{O}_{\mathfrak{p}} A e \subseteq \mathfrak{q} \mathcal{O}_{\mathfrak{p}} A
$$

Since $e$ and $(1-e)$ are idempotents, we get

$$
e \mathcal{O}_{\mathfrak{p}} A(1-e) \subseteq \mathfrak{q}^{\prime} e \mathcal{O}_{\mathfrak{p}} A(1-e) \text { and }(1-e) \mathcal{O}_{\mathfrak{p}} A e \subseteq \mathfrak{q}^{\prime}(1-e) \mathcal{O}_{\mathfrak{p}} A e .
$$

However, $\mathfrak{q}^{\prime}$ is contained in the maximal ideal $\mathfrak{p}^{\prime}$ of $\mathcal{O}_{\mathfrak{p}}$. By Nakayama's lemma, we obtain that

$$
e \mathcal{O}_{\mathfrak{p}} A(1-e)=(1-e) \mathcal{O}_{\mathfrak{p}} A e=\{0\} .
$$

We deduce that

$$
\mathcal{O}_{\mathfrak{p}} A=e \mathcal{O}_{\mathfrak{p}} A e \oplus(1-e) \mathcal{O}_{\mathfrak{p}} A(1-e)
$$

and consequently, $e$ is central. 
Proposition 2.1.13 If $K=F$, then the morphism

$$
\pi_{\mathfrak{q}}: Z \mathcal{O}_{\mathfrak{p}} A \rightarrow Z\left(\mathcal{O}_{\mathfrak{p}} / \mathfrak{q}^{\prime}\right) A
$$

induces a bijection between the set of blocks of $\mathcal{O}_{\mathfrak{p}} A$ and the set of blocks of $\left(\mathcal{O}_{\mathfrak{p}} / \mathfrak{q}^{\prime}\right) A$.

Proof: From now on, the symbol ${ }^{\wedge}$ will stand for $\mathfrak{p}$-adic completion. It is immediate that $\pi_{\mathfrak{q}}$ sends a block of $\mathcal{O}_{\mathfrak{p}} A$ to a sum of blocks of $\left(\mathcal{O}_{\mathfrak{p}} / \mathfrak{q}^{\prime}\right) A$. Now let $\bar{e}$ be a block of $\left(\mathcal{O}_{\mathfrak{p}} / \mathfrak{q}^{\prime}\right) A$. Following Theorem 1.3.3, all Noetherian local rings are contained in their completions. By Corollary 1.3.5, the completion of $\mathcal{O}_{\mathfrak{p}} / \mathfrak{q}^{\prime}$ with respect to the $\mathfrak{p}$-adic topology is $\hat{\mathcal{O}}_{\mathfrak{p}} / \mathfrak{q} \hat{\mathcal{O}}_{\mathfrak{p}}$. Thus, $\bar{e} \in\left(\hat{\mathcal{O}}_{\mathfrak{p}} / \mathfrak{q} \hat{\mathcal{O}}_{\mathfrak{p}}\right) A \cong \hat{\mathcal{O}}_{\mathfrak{p}} A / \mathfrak{q} \hat{\mathcal{O}}_{\mathfrak{p}} A$. In particular, $\bar{e} \in Z\left(\hat{\mathcal{O}}_{\mathfrak{p}} / \mathfrak{q} \hat{\mathcal{O}}_{\mathfrak{p}}\right) A$. By the theorems of lifting idempotents (cf., for example, 62, Theorem 3.2) and the lemma above, $\bar{e}$ is lifted to a central idempotent $e$ in $\hat{\mathcal{O}}_{\mathfrak{p}} A$. Following Theorem 2.1.6, $e$ is a sum of blocks of $\hat{\mathcal{O}}_{\mathfrak{p}} A$. Since the algebra $K A$ is split semisimple, the blocks of $\hat{\mathcal{O}}_{\mathfrak{p}} A$ belong to $K A$. By Theorem 1.3.6, $K \cap \hat{\mathcal{O}}_{\mathfrak{p}}=\mathcal{O}_{\mathfrak{p}}$ and thus $e$ is a sum of primitive idempotents of $\mathcal{O}_{\mathfrak{p}} A$ which belong to $Z \hat{\mathcal{O}}_{\mathfrak{p}} A$. Since $\mathcal{O}_{\mathfrak{p}} A \cap Z \hat{\mathcal{O}}_{\mathfrak{p}} A=Z \mathcal{O}_{\mathfrak{p}} A$, we deduce that $\bar{e}$ is lifted to a sum of blocks of $\mathcal{O}_{\mathfrak{p}} A$ and this provides the block bijection.

The following well-known result on blocks is the application of our Proposition 2.1 .13 to the case $\mathfrak{q}=\mathfrak{p}$.

Corollary 2.1.14 If $K=F$ and $k_{\mathfrak{p}}$ is the residue field of $\mathcal{O}_{\mathfrak{p}}$, then the morphism

$$
\pi_{\mathfrak{p}}: Z \mathcal{O}_{\mathfrak{p}} A \rightarrow Z k_{\mathfrak{p}} A
$$

induces a bijection between the set of blocks of $\mathcal{O}_{\mathfrak{p}} A$ and the set of blocks of $k_{\mathfrak{p}} A$.

\subsubsection{Blocks and central characters}

Since $K A$ is a split semisimple $K$-algebra, we have that

$$
K A \cong \prod_{\chi \in \operatorname{Irr}(K A)} M_{\chi}
$$

where $M_{\chi}$ is a matrix algebra isomorphic to $\operatorname{Mat}_{\chi(1)}(K)$.

Recall that $A$ is of finite type and thus integral over $\mathcal{O}([12], \S 1$, Def. 2$)$. The map $\pi_{\chi}: K A \rightarrow M_{\chi}$, restricted to $Z K A$, defines a map $\omega_{\chi}: Z K A \rightarrow K$ (by Schur's lemma), which in turn, restricted to $Z A$, defines the morphism

$$
\omega_{\chi}: Z A \rightarrow \mathcal{O}_{K}
$$


where $\mathcal{O}_{K}$ denotes the integral closure of $\mathcal{O}$ in $K$.

Now let $\mathfrak{p}$ be a prime ideal of $\mathcal{O}$. In the case where $\mathcal{O}_{\mathfrak{p}}$ is a discrete valuation ring (for example, when $\mathfrak{p}$ is a prime ideal of height 1 ), we have the following result which is proven later in this chapter, Proposition 2.4.16. For a different approach to its proof, see [18], Proposition 1.18.

Proposition 2.1.15 Suppose that $\mathcal{O}_{\mathfrak{p}}$ is a discrete valuation ring with unique maximal ideal $\mathfrak{p}^{\prime}:=\mathfrak{p} \mathcal{O}_{\mathfrak{p}}$ and $K=F$. Two characters $\chi, \chi^{\prime} \in \operatorname{Irr}(K A)$ belong to the same block of $\mathcal{O}_{\mathfrak{p}} A$ if and only if

$$
\omega_{\chi}(z) \equiv \omega_{\chi^{\prime}}(z) \bmod \mathfrak{p}^{\prime} \text { for all } z \in Z \mathcal{O}_{\mathfrak{p}} A .
$$

\subsection{Symmetric algebras}

Let $\mathcal{O}$ be a ring and let $A$ be an $\mathcal{O}$-algebra. Suppose again that the assumptions 2.1.7 are satisfied.

Definition 2.2.1 A trace function on $A$ is an $\mathcal{O}$-linear map $t: A \rightarrow \mathcal{O}$ such that $t(a b)=t(b a)$ for all $a, b \in A$.

Definition 2.2.2 We say that a trace function $t: A \rightarrow \mathcal{O}$ is a symmetrizing form on $A$ or that $A$ is a symmetric algebra if the morphism

$$
\hat{t}: A \rightarrow \operatorname{Hom}_{\mathcal{O}}(A, \mathcal{O}), a \mapsto(x \mapsto \hat{t}(a)(x):=t(a x))
$$

is an isomorphism of $A$-modules- $A$.

Example 2.2.3 In the case where $\mathcal{O}=\mathbb{Z}$ and $A=\mathbb{Z}[G]$ ( $G$ a finite group), we can define the following symmetrizing form ("canonical") on $A$

$$
t: \mathbb{Z}[G] \rightarrow \mathbb{Z}, \sum_{g \in G} a_{g} g \mapsto a_{1},
$$

where $a_{g} \in \mathbb{Z}$ for all $g \in G$.

Since $A$ is a free $\mathcal{O}$-module of finite rank, we have the following isomorphism

$$
\begin{aligned}
& \operatorname{Hom}_{\mathcal{O}}(A, \mathcal{O}) \otimes_{\mathcal{O}} A \stackrel{\sim}{\rightarrow} \operatorname{Hom}_{\mathcal{O}}(A, A) \\
& \varphi \otimes a \quad \mapsto \quad(x \mapsto \varphi(x) a) .
\end{aligned}
$$

Composing it with the isomorphism

$$
\begin{array}{ccc}
A \otimes_{\mathcal{O}} A & \sim & \operatorname{Hom}_{\mathcal{O}}(A, \mathcal{O}) \otimes_{\mathcal{O}} A \\
a \otimes b & \mapsto & \hat{t}(a) \otimes b,
\end{array}
$$

we obtain an isomorphism

$$
A \otimes_{\mathcal{O}} A \stackrel{\sim}{\rightarrow} \operatorname{Hom}_{\mathcal{O}}(A, A) .
$$


Definition 2.2.4 We denote by $C_{A}$ and we call Casimir of $(A, t)$ the inverse image of $\mathrm{id}_{A}$ by the above isomorphism.

Example 2.2.5 In the case where $\mathcal{O}=\mathbb{Z}, A=\mathbb{Z}[G]$ ( $G$ a finite group) and $t$ is the canonical symmetrizing form, we have $C_{\mathbb{Z}[G]}=\sum_{g \in G} g^{-1} \otimes g$.

More generally, if $\left(e_{i}\right)_{i \in I}$ is a basis of $A$ over $\mathcal{O}$ and $\left(e_{i}^{\prime}\right)_{i \in I}$ is the dual basis with respect to $t$ (i.e., $\left.t\left(e_{i} e_{j}^{\prime}\right)=\delta_{i j}\right)$, then

$$
C_{A}=\sum_{i \in I} e_{i}^{\prime} \otimes e_{i}
$$

In this case, let us denote by $c_{A}$ the image of $C_{A}$ by the multiplication $A \otimes A \rightarrow A$, i.e., $c_{A}=\sum_{i \in I} e_{i}^{\prime} e_{i}$. It is easy to check (see also [20], 7.9) the following properties of the Casimir element:

Lemma 2.2.6 For all $a \in A$, we have

1. $\sum_{i} a e_{i}^{\prime} \otimes e_{i}=\sum_{i} e_{i} \otimes e_{i}^{\prime} a$.

2. $a C_{A}=C_{A}$ a. Consequently, $c_{A} \in Z A$.

3. $a=\sum_{i} t\left(a e_{i}^{\prime}\right) e_{i}=\sum_{i} t\left(a e_{i}\right) e_{i}^{\prime}=\sum_{i} t\left(e_{i}^{\prime}\right) e_{i} a=\sum_{i} t\left(e_{i}\right) e_{i}^{\prime} a$.

If $\tau: A \rightarrow \mathcal{O}$ is a linear form, we denote by $\tau^{\vee}$ its inverse image by the isomorphism $\hat{t}$, i.e., $\tau^{\vee}$ is the element of $A$ such that

$$
t\left(\tau^{\vee} a\right)=\tau(a) \text { for all } a \in A .
$$

The element $\tau^{\vee}$ has the following properties:

\section{Lemma 2.2.7}

1. $\tau$ is a trace function if and only if $\tau^{\vee} \in Z A$.

2. We have $\tau^{\vee}=\sum_{i} \tau\left(e_{i}^{\prime}\right) e_{i}=\sum_{i} \tau\left(e_{i}\right) e_{i}^{\prime}$ and more generally, for all $a \in A$, we have $\tau^{\vee} a=\sum_{i} \tau\left(e_{i}^{\prime} a\right) e_{i}=\sum_{i} \tau\left(e_{i} a\right) e_{i}^{\prime}$.

\section{Proof:}

1. Recall that $t$ is a trace function. Let $a \in A$. For all $x \in A$, we have

$$
\hat{t}\left(\tau^{\vee} a\right)(x)=t\left(\tau^{\vee} a x\right)=\tau(a x)
$$

and

$$
\hat{t}\left(a \tau^{\vee}\right)(x)=t\left(a \tau^{\vee} x\right)=t\left(\tau^{\vee} x a\right)=\tau(x a)
$$


If $\tau$ is a trace function, then $\tau(a x)=\tau(x a)$ and hence, $\hat{t}\left(\tau^{\vee} a\right)=\hat{t}\left(a \tau^{\vee}\right)$. Since $\hat{t}$ is an isomorphism, we obtain that $\tau^{\vee} a=a \tau^{\vee}$ and thus $\tau^{\vee} \in Z A$.

Now if $\tau^{\vee} \in Z A$ and $a, b \in A$, then

$$
\tau(a b)=t\left(\tau^{\vee} a b\right)=t\left(b \tau^{\vee} a\right)=t\left(b a \tau^{\vee}\right)=t\left(\tau^{\vee} b a\right)=\tau(b a) .
$$

2. It derives from property 3 of Lemma 2.2.6 and the definition of $\tau^{\vee}$.

Let $\chi_{\text {reg }}$ be the character of the regular representation of $A$, i.e., the linear form on $A$ defined as

$$
\chi_{\mathrm{reg}}(a):=\operatorname{tr}_{A / \mathcal{O}}\left(\lambda_{a}\right),
$$

where $\lambda_{a}: A \rightarrow A, x \mapsto a x$, is the endomorphism of left multiplication by $a$.

Proposition 2.2.8 We have $\chi_{\mathrm{reg}}^{\vee}=c_{A}$.

Proof: $\quad$ Let $a \in A$. The inverse image of $\lambda_{a}$ by the isomorphism $A \otimes_{\mathcal{O}} A \stackrel{\sim}{\rightarrow} \operatorname{Hom}_{\mathcal{O}}(A, A)$ is $a C_{A}$ (by definition of the Casimir). Hence,

$$
\lambda_{a}=\left(x \mapsto \sum_{i} \hat{t}\left(e_{i}^{\prime} a\right)(x) e_{i}\right)=\left(x \mapsto \sum_{i} t\left(e_{i}^{\prime} a x\right) e_{i}\right)
$$

and

$$
\operatorname{tr}_{A / \mathcal{O}}\left(\lambda_{a}\right)=\sum_{i} t\left(e_{i}^{\prime} a e_{i}\right)=t\left(a \sum_{i} e_{i}^{\prime} e_{i}\right)=t\left(a c_{A}\right)=t\left(c_{A} a\right)
$$

Therefore, for all $a \in A$, we have $\chi_{\mathrm{reg}}(a)=t\left(c_{A} a\right)$, i.e., $\chi_{\mathrm{reg}}^{\vee}=c_{A}$.

If $A$ is a symmetric algebra with a symmetrizing form $t$, we obtain a symmetrizing form $t^{K}$ on $K A$ by extension of scalars. Every irreducible character $\chi \in \operatorname{Irr}(K A)$ is a trace function on $K A$ and thus we can define $\chi^{\vee} \in Z K A$.

Definition 2.2.9 For all $\chi \in \operatorname{Irr}(K A)$, we call Schur element of $\chi$ with respect to $t$ and denote by $s_{\chi}$ the element of $K$ defined by

$$
s_{\chi}:=\omega_{\chi}\left(\chi^{\vee}\right) .
$$

Proposition 2.2.10 For all $\chi \in \operatorname{Irr}(K A), s_{\chi} \in \mathcal{O}_{K}$.

The proof of the above result will be given in Proposition 2.4.6. 
Example 2.2.11 Let $\mathcal{O}:=\mathbb{Z}, A:=\mathbb{Z}[G]$ ( $G$ a finite group) and $t$ the canonical symmetrizing form. If $K$ is an algebraically closed field of characteristic 0 , then $K A$ is a split semisimple algebra and $s_{\chi}=|G| / \chi(1)$ for all $\chi \in \operatorname{Irr}(K A)$. Because of the integrality of the Schur elements, we must have $|G| / \chi(1) \in \mathbb{Z}=\mathbb{Z}_{K} \cap \mathbb{Q}$ for all $\chi \in \operatorname{Irr}(K A)$. Thus, we have shown that $\chi(1)$ divides $|G|$.

The following properties of the Schur elements can be derived easily from the above (see also [17, [32, [33, ,34, 20])

\section{Proposition 2.2.12}

1. We have

$$
t=\sum_{\chi \in \operatorname{Irr}(K A)} \frac{1}{s_{\chi}} \chi .
$$

2. For all $\chi \in \operatorname{Irr}(K A)$, the central primitive idempotent associated with $\chi$ is

$$
e_{\chi}=\frac{1}{s_{\chi}} \chi^{\vee}=\frac{1}{s_{\chi}} \sum_{i \in I} \chi\left(e_{i}^{\prime}\right) e_{i}
$$

3. For all $\chi \in \operatorname{Irr}(K A)$, we have

$$
s_{\chi} \chi(1)=\sum_{i \in I} \chi\left(e_{i}^{\prime}\right) \chi\left(e_{i}\right) \text { and } s_{\chi} \chi(1)^{2}=\chi\left(\sum_{i \in I} e_{i}^{\prime} e_{i}\right)=\chi\left(\chi_{\mathrm{reg}}^{\vee}\right) .
$$

Corollary 2.2.13 The blocks of $A$ are the non-empty subsets $B$ of $\operatorname{Irr}(K A)$ minimal with respect to the property

$$
\sum_{\chi \in B} \frac{1}{s_{\chi}} \chi(a) \in \mathcal{O} \text { for all } a \in A .
$$

\subsection{Twisted symmetric algebras of finite groups}

This part is an adaptation of the section "Symmetric algebras of finite groups" of [18] to a more general case.

Let $A$ be an $\mathcal{O}$-algebra such that the assumptions 2.1.7 are satisfied with a symmetrizing form $t$. Let $\bar{A}$ be a subalgebra of $A$ free and of finite rank as $\mathcal{O}$-module.

We denote by $\bar{A}^{\perp}$ the orthogonal of $\bar{A}$ with respect to $t$, i.e., the sub- $\bar{A}$ module- $\bar{A}$ of $A$ defined as

$$
\bar{A}^{\perp}:=\{a \in A \mid(\forall \bar{a} \in \bar{A})(t(a \bar{a})=0)\} .
$$




\section{Proposition 2.3.1}

1. The restriction of $t$ to $\bar{A}$ is a symmetrizing form for $\bar{A}$ if and only if $\bar{A} \oplus \bar{A}^{\perp}=A$. In this case the projection of $A$ onto $\bar{A}$ parallel to $\bar{A}^{\perp}$ is the map

$$
\operatorname{Br}_{\bar{A}}^{A}: A \rightarrow \bar{A} \text { such that } t\left(\operatorname{Br}_{\bar{A}}^{A}(a) \bar{a}\right)=t(a \bar{a}) \text { for all } a \in A \text { and } \bar{a} \in \bar{A} \text {. }
$$

2. If the restriction of $t$ to $\bar{A}$ is a symmetrizing form for $\bar{A}$, then $\bar{A}^{\perp}$ is the sub- $\bar{A}$-module- $\bar{A}$ of $A$ defined by the following two properties:

(a) $A=\bar{A} \oplus \bar{A}^{\perp}$,

(b) $\bar{A}^{\perp} \subseteq$ Kert.

\section{Proof:}

1. Let us denote by $\bar{t}$ the restriction of $t$ to $\bar{A}$. Suppose that $\bar{t}$ is a symmetrizing form for $\bar{A}$. Let $a \in A$. Then $\hat{t}(a):=(x \mapsto t(a x)) \in$ $\operatorname{Hom}_{\mathcal{O}}(A, \mathcal{O})$. The restriction of $\hat{t}(a)$ to $\bar{A}$ belongs to $\operatorname{Hom}_{\mathcal{O}}(\bar{A}, \mathcal{O})$ and therefore, there exists $\bar{a} \in \bar{A}$ such that $\bar{t}(\bar{a} \bar{x})=t(\bar{a} \bar{x})=t(a \bar{x})$ for all $\bar{x} \in \bar{A}$. Thus $a-\bar{a} \in \bar{A}^{\perp}$ and since $a=\bar{a}+(a-\bar{a})$, we obtain that $A=\bar{A}+\bar{A}^{\perp}$. If $\bar{a} \in \bar{A} \cap \bar{A}^{\perp}$, then we have $\hat{\bar{t}}(\bar{a})=0 \in \operatorname{Hom}_{\mathcal{O}}(\bar{A}, \mathcal{O})$. Since $\hat{\bar{t}}$ is an isomorphism, we deduce that $\bar{a}=0$. Therefore, $A=\bar{A} \oplus \bar{A}^{\perp}$ and the definition of $\operatorname{Br}_{\bar{A}}^{A}$ is immediate.

Now suppose that $A=\bar{A} \oplus \bar{A}^{\perp}$. We will show that the map

$$
\begin{aligned}
\hat{\bar{t}}: \bar{A} & \rightarrow \operatorname{Hom}_{\mathcal{O}}(\bar{A}, \mathcal{O}) \\
\bar{A} & \mapsto(\bar{x} \mapsto \bar{t}(\bar{a} \bar{x})=t(\bar{a} \bar{x}))
\end{aligned}
$$

is an isomorphism of $\bar{A}$-modules- $\bar{A}$. The map $\hat{\bar{t}}$ is obviously injective, because $\hat{\bar{t}}(\bar{a})=0$ implies that $\bar{a} \in \bar{A} \cap \bar{A}^{\perp}$ and thus $\bar{a}=0$. Now let $\bar{f}$ be an element of $\operatorname{Hom}_{\mathcal{O}}(\bar{A}, \mathcal{O})$. The map $\bar{f}$ can be extended to a map $f \in \operatorname{Hom}_{\mathcal{O}}(A, \mathcal{O})$ such that $f(a)=\bar{f}\left(\operatorname{Br}_{\bar{A}}^{A}(a)\right)$ for all $a \in A$, where $\mathrm{Br}_{\bar{A}}^{A}$ denotes the projection of $A$ onto $\bar{A}$ parallel to $\bar{A}^{\perp}$. Since $t$ is a symmetrizing form for $A$, there exists $a \in A$ such that $\hat{t}(a)=f$, i.e., $t(a x)=f(x)$ for all $x \in A$. Consequently, if $\bar{x} \in \bar{A}$, we have

$$
t\left(\operatorname{Br}_{\bar{A}}^{A}(a) \bar{x}\right)=t(a \bar{x})=f(\bar{x})=\bar{f}(\bar{x})
$$

and thus $\hat{\bar{t}}\left(\operatorname{Br}_{\bar{A}}^{A}(a)\right)=\bar{f}$. Hence, $\hat{\bar{t}}$ is surjective. 
2. Let $B$ be a sub- $\bar{A}$-module- $\bar{A}$ of $A$ such that $A=\bar{A} \oplus B$ and $B \subseteq$ Kert. Let $b \in B$. For all $\bar{a} \in \bar{A}$, we have $b \bar{a} \in B \subseteq$ Kert and thus $t(b \bar{a})=0$. Hence $B \subseteq \bar{A}^{\perp}$. Since the restriction of $t$ to $\bar{A}$ is a symmetrizing form for $\bar{A}$, we also have $A=\bar{A} \oplus \bar{A}^{\perp}$. Now let $a \in \bar{A}^{\perp}$. Since $A=\bar{A} \oplus B$, there exist $\bar{a} \in \bar{A}$ and $b \in B$ such that $a=\bar{a}+b$. Since $b \in \bar{A}^{\perp}$, we must have $a=b \in B$ and therefore, $B=\bar{A}^{\perp}$.

Example 2.3.2 In the case where $\mathcal{O}=\mathbb{Z}$ and $A=\mathbb{Z}[G]$ (G a finite group), let $\bar{A}:=\mathbb{Z}[\bar{G}]$ be the algebra of a subgroup $\bar{G}$ of $G$. Then the morphism $\operatorname{Br}_{\bar{A}}^{A}$ is the projection given by

$$
\begin{cases}g \mapsto g, & \text { if } g \in \bar{G} \\ g \mapsto 0, & \text { if } g \notin \bar{G}\end{cases}
$$

Definition 2.3.3 Let $A$ be a symmetric $\mathcal{O}$-algebra with symmetrizing form t. Let $\bar{A}$ be a subalgebra of $A$. We say that $\bar{A}$ is a symmetric subalgebra of $A$, if it satisfies the following two conditions:

1. $\bar{A}$ is free (of finite rank) as an $\mathcal{O}$-module and the restriction $\operatorname{Res}_{\bar{A}}^{A}(t)$ of the form $t$ to $\bar{A}$ is a symmetrizing form on $\bar{A}$,

2. A is free (of finite rank) as an $\bar{A}$-module for the action of left multiplication by the elements of $\bar{A}$.

From now on, let us suppose that $\bar{A}$ is a symmetric subalgebra of $A$ and set $\bar{t}:=\operatorname{Res}_{\bar{A}}^{A}(t)$. We denote by

$$
\operatorname{Ind}_{\bar{A}}^{A}:_{\bar{A}} \bmod \rightarrow_{A} \bmod \text { and } \operatorname{Res}_{\bar{A}}^{A}:_{A} \bmod \rightarrow_{\bar{A}} \bmod
$$

the functors defined as usual by

$$
\operatorname{Ind}_{\bar{A}}^{A}:=A \otimes_{\bar{A}}-\text { where } A \text { is viewed as an } A \text {-module- } \bar{A}
$$

and

$$
\operatorname{Res}_{\bar{A}}^{A}:=A \otimes_{A}-\text { where } A \text { is viewed as an } \bar{A} \text {-module- } A \text {. }
$$

Since $A$ is free as $\bar{A}$-module and as module- $\bar{A}$, the functors $\operatorname{Res}_{\bar{A}}^{A}$ and $\operatorname{Ind}_{\bar{A}}^{A}$ are adjoint from both sides.

Moreover, let $K$ be a finite Galois extension of the field of fractions of $\mathcal{O}$ such that the algebras $K A$ and $K \bar{A}$ are both split semisimple.

We denote by $\langle-,-\rangle_{K A}$ the scalar product on the $K$-vector space of trace functions for which the family $(\chi)_{\chi \in \operatorname{Irr}(K A)}$ is orthonormal and $\langle-,-\rangle_{K \bar{A}}$ the 
scalar product on the $K$-vector space of trace functions for which the family $(\bar{\chi})_{\bar{\chi} \in \operatorname{Irr}(K \bar{A})}$ is orthonormal.

Since the functors $\operatorname{Res}_{\bar{A}}^{A}$ and $\operatorname{Ind}_{\bar{A}}^{A}$ are adjoint from both sides, we obtain the Frobenius reciprocity formula:

$$
\left\langle\chi, \operatorname{Ind}_{K \bar{A}}^{K A}(\bar{\chi})\right\rangle_{K A}=\left\langle\operatorname{Res}_{K \bar{A}}^{K A}(\chi), \bar{\chi}\right\rangle_{K \bar{A}} .
$$

For every element $\chi \in \operatorname{Irr}(K A)$, let

$$
\operatorname{Res}_{K \bar{A}}^{K A}(\chi)=\sum_{\bar{\chi} \in \operatorname{Irr}(K \bar{A})} m_{\chi, \bar{\chi}} \bar{\chi}\left(\text { where } m_{\chi, \bar{\chi}} \in \mathbb{N}\right) .
$$

Frobenius reciprocity implies that, for all $\bar{\chi} \in \operatorname{Irr}(K \bar{A})$,

$$
\operatorname{Ind}_{K \bar{A}}^{K A}(\bar{\chi})=\sum_{\chi \in \operatorname{Irr}(K A)} m_{\chi, \bar{\chi}} \chi
$$

The following property is immediate.

Lemma 2.3.4 For $\chi \in \operatorname{Irr}(K A)$ and $\bar{\chi} \in \operatorname{Irr}(K \bar{A})$, let $e(\chi)$ and $\bar{e}(\bar{\chi})$ be respectively the block-idempotents of $K A$ and $K \bar{A}$ associated with $\chi$ and $\bar{\chi}$. The following conditions are equivalent:

(i) $m_{\chi, \bar{\chi}} \neq 0$,

(ii) $e(\chi) \bar{e}(\bar{\chi}) \neq 0$.

For all $\bar{\chi} \in \operatorname{Irr}(K \bar{A})$, we set

$$
\operatorname{Irr}(K A, \bar{\chi}):=\left\{\chi \in \operatorname{Irr}(K A) \mid m_{\chi, \bar{\chi}} \neq 0\right\},
$$

and for all $\chi \in \operatorname{Irr}(K A)$,

$$
\operatorname{Irr}(K \bar{A}, \chi):=\left\{\bar{\chi} \in \operatorname{Irr}(K \bar{A}) \mid m_{\chi, \bar{\chi}} \neq 0\right\} .
$$

We denote respectively by $s_{\chi}$ and $s_{\bar{\chi}}$ the Schur elements of $\chi$ and $\bar{\chi}$ ( with respect to the symmetrizing forms $t$ for $A$ and $\bar{t}$ for $\bar{A}$ ).

Lemma 2.3.5 For all $\bar{\chi} \in \operatorname{Irr}(K \bar{A})$ we have

$$
\frac{1}{s_{\bar{\chi}}}=\sum_{\chi \in \operatorname{Irr}(K A, \bar{\chi})} \frac{m_{\chi, \bar{\chi}}}{s_{\chi}} .
$$


Proof: It derives from the relations

$$
t=\sum_{\chi \in \operatorname{Irr}(K A)} \frac{1}{s_{\chi}} \chi, \bar{t}=\sum_{\bar{\chi} \in \operatorname{Irr}(K \bar{A})} \frac{1}{s_{\bar{\chi}}} \bar{\chi}, \bar{t}=\operatorname{Res}_{\bar{A}}^{A}(t) .
$$

In the next chapters, we will work on the Hecke algebras of complex reflection groups, which, under certain assumptions, are symmetric. Sometimes the Hecke algebra of a group $W$ appears as a symmetric subalgebra of the Hecke algebra of another group $W^{\prime}$, which contains $W$. Since we will be mostly interested in the determination of the blocks of these algebras, it would be helpful, if we could obtain the blocks of the former from the blocks of the latter or vice versa. This is possible with the use of a generalization of some classical results known as "Clifford theory".

"Clifford theory" was originally developed by A. H. Clifford in 1937 for finite group algebras over a field (cf. [26]): if $G$ is a finite group, $F$ is a field and $N$ is a normal subgroup of $G$, then important information concerning simple and indecomposable $K G$-modules can be obtained by applying (perhaps repeatedly) three basic operations: (a) restriction to $F N$, (b) extension from $F N$, (c) induction from $F N$. In the past twenty years, the theory has enjoyed vigorous development. The foundations have been strengthened and reorganized from new points of view and Clifford's results have been generalized to various types of algebras (cf., for example, [28]). Here we will generalize these results to the case of, what we are about to define as, the twisted symmetric algebras of finite groups and in particular, of finite cyclic groups.

Definition 2.3.6 Let $A$ be a symmetric $\mathcal{O}$-algebra with symmetrizing form t. We say that $A$ is the twisted symmetric algebra of a finite group $G$ over the subalgebra $\bar{A}$, if the following conditions are satisfied:

1. $\bar{A}$ is a symmetric subalgebra of $A$.

2. There exists a family $\left\{A_{g} \mid g \in G\right\}$ of $\mathcal{O}$-submodules of $A$ such that

(a) $A=\bigoplus_{g \in G} A_{g}$

(b) $A_{g} A_{h}=A_{g h}$ for all $g, h \in G$,

(c) $A_{1}=\bar{A}$,

(d) $t\left(A_{g}\right)=0$ for all $g \in G \backslash\{1\}$. 
If that is the case, then Proposition 2.3.1 implies that

$$
\bigoplus_{g \in G \backslash\{1\}} A_{g}=\bar{A}^{\perp} .
$$

Proposition 2.3.7 Let $A$ be a symmetric $\mathcal{O}$-algebra with symmetrizing form t. The algebra $A$ is the twisted symmetric algebra of a finite group $G$ over the subalgebra $\bar{A}$ if and only if the following conditions are satisfied:

1. $\bar{A}$ is a symmetric subalgebra of $A$.

2. There exists a family $\left\{a_{g} \mid g \in G\right\}$ of invertible elements of A such that

(a) $A=\bigoplus_{g \in G} a_{g} \bar{A}$,

(b) $a_{g} \bar{A}=\bar{A} a_{g}$ for all $g \in G$,

(c) $a_{g} a_{h} \bar{A}=a_{g h} \bar{A}$ for all $g, h \in G$,

(d) $a_{1}=1$

(e) $a_{g} \in \bar{A}^{\perp}$ for all $g \in G \backslash\{1\}$.

Proof: $\quad$ If $A$ is the twisted symmetric algebra of a finite group $G$ over the subalgebra $\bar{A}$, then $\bar{A}$ is a symmetric subalgebra of $A$ and there exists a family $\left\{A_{g} \mid g \in G\right\}$ of $\mathcal{O}$-submodules of $A$ which satisfy the conditions (a)-(d) of Definition 2.3.6. Let $g \in G$. Then, by property (b), $A_{g} A_{g^{-1}}=A_{1}=\bar{A}$. Since $1 \in \bar{A}$, there exists $a_{g} \in A_{g}$ and $a_{g^{-1}} \in A_{g^{-1}}$ such that $a_{g} a_{g^{-1}}=1$. Now, if $x \in A_{g}$, then $x=a_{g} a_{g}^{-1} x \in a_{g} A_{g^{-1}} A_{g}=a_{g} \bar{A}$ and thus, $A_{g} \subseteq a_{g} \bar{A}$. Property (b) implies the inverse inclusion. In the same way, we show that $A_{g}=\bar{A} a_{g}$. Hence, we obtain that there exists a family $\left\{a_{g} \mid g \in G\right\}$ of invertible elements of $A$ such that

$$
A=\bigoplus_{g \in G} a_{g} \bar{A} \text { and } a_{g} \bar{A}=\bar{A} a_{g} \text { for all } g \in G .
$$

We can choose $a_{1}:=1$. Moreover, for all $g, h \in G$, we have $a_{g} a_{h} \in A_{g} A_{h}=$ $A_{g h}$. Since $a_{g} a_{h}$ is a unit in $A$, we obtain that $A_{g h}=a_{g} a_{h} \bar{A}$, i.e., $a_{g h} \bar{A}=$ $a_{g} a_{h} \bar{A}$. Finally, for all $g \in G \backslash\{1\}$ and $\bar{a} \in \bar{A}$, we have $a_{g} \bar{a} \in a_{g} \bar{A}=A_{g}$ and property (d) implies that $t\left(a_{g} \bar{a}\right)=0$. Thus, $a_{g} \in \bar{A}^{\perp}$ for all $g \in G \backslash\{1\}$.

Now, let us suppose that the conditions 1 and 2 are satisfied. For all $g \in G$, we set $A_{g}:=a_{g} \bar{A}=\bar{A} a_{g}$. We only need to show that the family $\left\{A_{g} \mid g \in G\right\}$ satisfies the conditions (b), (c) and (d) of Definition 2.3.6. Obviously, $A_{1}=a_{1} \bar{A}=1 \bar{A}=\bar{A}$. Moreover, if $g \in G \backslash\{1\}$ and $x \in A_{g}$, then there exists $\bar{a} \in \bar{A}$ such that $x=a_{g} \bar{a}$. Since $a_{g} \in \bar{A}^{\perp}$, we obtain that $t(x)=t\left(a_{g} \bar{a}\right)=0$. Hence, $t\left(A_{g}\right)=0$ for all $g \in G \backslash\{1\}$. It remains to show that $A_{g} A_{h}=A_{g h}$ for all $g, h \in G$. We have $A_{g} A_{h}=\left(a_{g} \bar{A}\right)\left(a_{h} \bar{A}\right)=$ 
$a_{g}\left(\bar{A} a_{h}\right) \bar{A}=a_{g}\left(a_{h} \bar{A}\right) \bar{A}=a_{g} a_{h} \bar{A}=a_{g h} \bar{A}=A_{g h}$.

From now on, let $(A, t)$ be the twisted symmetric algebra of a finite group $G$ over the subalgebra $\bar{A}$. Due to the proposition above, for all $g \in G$, there exists $a_{g} \in A_{g} \cap A^{\times}$such that $A_{g}=a_{g} \bar{A}=\bar{A} a_{g}$. We fix a system of representatives $\operatorname{Rep}(A / \bar{A}):=\left\{a_{g} \mid g \in G\right\}$.

Proposition 2.3.8 Let $\left(\bar{e}_{i}\right)_{i \in I}$ be a basis of $\bar{A}$ over $\mathcal{O}$ and $\left(\bar{e}_{i}^{\prime}\right)_{i \in I}$ its dual with respect to the symmetrizing form $\bar{t}$. Then the families

$$
\left(\bar{e}_{i} a_{g}\right)_{i \in I, a_{g} \in \operatorname{Rep}(A / \bar{A})} \text { and }\left(a_{g}^{-1} \bar{e}_{i}^{\prime}\right)_{i \in I, a_{g} \in \operatorname{Rep}(A / \bar{A})}
$$

are two $\mathcal{O}$-bases of $A$ dual to each other.

\subsubsection{Action of $G$ on $Z \bar{A}$}

Lemma 2.3.9 Let $\bar{a} \in Z \bar{A}$ and $g \in G$. There exists a unique element $g(\bar{a})$ of $\bar{A}$ satisfying

$$
g(\bar{a}) x_{g}=x_{g} \bar{a} \text { for all } x_{g} \in A_{g} .
$$

In particular, $g(\bar{a})=a_{g} \bar{a} a_{g}^{-1}$.

Proof: $\quad$ For all $x_{g} \in A_{g}$, we have $a_{g}^{-1} x_{g} \in \bar{A}$. Set $g(\bar{a}):=a_{g} \bar{a} a_{g}^{-1}$. Since $\bar{a} \in Z \bar{A}$, we obtain that $g(\bar{a}) x_{g}=a_{g} \bar{a} a_{g}^{-1} x_{g}=a_{g} a_{g}^{-1} x_{g} \bar{a}=x_{g} \bar{a}$. Now, let $y$ be another element of $A$ such that $y x_{g}=x_{g} \bar{a}$ for all $x_{g} \in A_{g}$. Then $y a_{g}=a_{g} \bar{a}$, whence $y=g(\bar{a})$. Therefore, $g(\bar{a})$ is the unique element of $A$ which satisfies $(\dagger)$.

Remark: Note that $g(\bar{a})$ does not depend on the choice of $a_{g}$.

Proposition 2.3.10 The map $\bar{a} \mapsto g(\bar{a})$ defines an action of $G$ as ring automorphism of $Z \bar{A}$.

Proof: $\quad$ Let $\bar{a} \in Z \bar{A}$ and $g \in G$. We will show that $g(\bar{a}) \in Z \bar{A}$. If $\bar{x} \in \bar{A}$, then $\bar{x} a_{g} \in A_{g}$ and we have

$$
\bar{x}\left(g(\bar{a}) a_{g}\right)=\bar{x}\left(a_{g} \bar{a}\right)=\left(\bar{x} a_{g}\right) \bar{a}=g(\bar{a})\left(\bar{x} a_{g}\right) .
$$

Multiplying both sides by $a_{g}^{-1}$, we obtain that

$$
\bar{x} g(\bar{a})=g(\bar{a}) \bar{x}
$$

and hence, $g(\bar{a}) \in Z \bar{A}$. 
Since $a_{1}=1$, we have $1_{G}(\bar{a})=\bar{a}$. If $g_{1}, g_{2} \in G$, then equation $(\dagger)$ gives

$$
g_{1}\left(g_{2}(\bar{a})\right) a_{g_{1}} a_{g_{2}}=a_{g_{1}} g_{2}(\bar{a}) a_{g_{2}}=a_{g_{1}} a_{g_{2}} \bar{a}
$$

Due to property (b) of Definition [2.3.6, the product $a_{g_{1}} a_{g_{2}}$ generates the submodule $A_{g_{1} g_{2}}$. Therefore, $g_{1}\left(g_{2}(\bar{a})\right) u=u \bar{a}$ for all $u \in A_{g_{1} g_{2}}$. By Lemma 2.3.9, we obtain that $\left(g_{1} g_{2}\right)(\bar{a})=g_{1}\left(g_{2}(\bar{a})\right)$.

Finally, let us fix $g \in G$. By definition, the map $\bar{a} \mapsto g(\bar{a})$ is an additive automorphism of $Z \bar{A}$. If $\bar{a}_{1}, \bar{a}_{2} \in Z \bar{A}$, then

$$
x_{g} \bar{a}_{1} \bar{a}_{2}=g\left(\bar{a}_{2}\right) x_{g} \bar{a}_{1}=g\left(\bar{a}_{1}\right) g\left(\bar{a}_{2}\right) x_{g} \text { for all } x_{g} \in A_{g} .
$$

By Lemma 2.3.9, we obtain that $g\left(\bar{a}_{1} \bar{a}_{2}\right)=g\left(\bar{a}_{1}\right) g\left(\bar{a}_{2}\right)$.

Now let $\bar{b}$ be a block(-idempotent) of $\bar{A}$. If $g \in G$, then $g(\bar{b})$ is also a block of $\bar{A}$. So we must have either $g(\bar{b})=\bar{b}$ or $g(\bar{b})$ orthogonal to $\bar{b}$. Set

$$
\operatorname{Tr}(G, \bar{b}):=\sum_{g \in G / G_{\bar{b}}} g(\bar{b})
$$

where $G_{\bar{b}}:=\{g \in G \mid g(\bar{b})=\bar{b}\}$. It is clear that

- $\bar{b}$ is a central idempotent of $\bigoplus_{g \in G_{\bar{b}}} A_{g}=: A_{G_{\bar{b}}}$,

- $\operatorname{Tr}(G, \bar{b})$ is a central idempotent of $A$.

From now on, let $b:=\operatorname{Tr}(G, \bar{b})$ and $x_{g} \in A_{g}$. We have

- $\bar{b} x_{g} \bar{b}= \begin{cases}x_{g} \bar{b}=\bar{b} x_{g}, & \text { if } g \in G_{\bar{b}} ; \\ 0, & \text { if } g \notin G_{\bar{b}},\end{cases}$

- $\bar{b} x_{g} b=\bar{b} x_{g}$ and $b x_{g} \bar{b}=x_{g} \bar{b}$.

Proposition 2.3.11 The applications

$$
\left\{\begin{array}{l}
b A \bar{b} \otimes_{A_{G_{\bar{b}}} \bar{b}} \bar{b} A b \rightarrow A b \\
b a_{g} \bar{a} \bar{b} \otimes \bar{b}^{\prime} a_{g^{\prime}} b \mapsto a_{\underline{g}} \bar{a} \bar{b} \bar{a}^{\prime} a_{g^{\prime}} \\
a b \mapsto \sum_{g \in G / G_{\bar{b}}} b a a_{g} \bar{b} \otimes \bar{b} a_{g}^{-1},
\end{array}\right.
$$

and

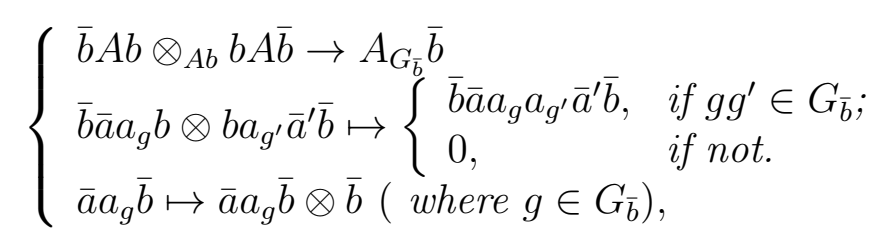


define isomorphisms inverse to each other

$$
b A \bar{b} \otimes_{A_{G_{\bar{b}}} \bar{b}} \bar{b} A b \stackrel{\leftrightarrow}{\leftrightarrow} A b \quad \text { and } \quad \bar{b} A b \otimes_{A b} b A \bar{b} \tilde{\leftrightarrow} A_{G_{\bar{b}}} \bar{b} .
$$

Therefore, $b A \bar{b}$ and $\bar{b} A b$ are Morita equivalent. In particular, the functors

$$
\operatorname{Ind}_{\bar{A}}^{A}=\left(b A \bar{b} \otimes_{A_{G_{\bar{b}}} \bar{b}}-\right) \quad \text { and } \quad \bar{b} \cdot \operatorname{Res}_{\bar{A}}^{A}=\left(\bar{b} A b \otimes_{A b}-\right)
$$

define category equivalences inverse to each other between ${ }_{A_{\bar{G}} \bar{b}}$ mod and ${ }_{A b} \bmod$.

\subsubsection{Multiplication of an $A$-module by an $\mathcal{O} G$-module}

Let $X$ be an $A$-module and $\rho: A \rightarrow \operatorname{End}_{\mathcal{O}}(X)$ be the structural morphism. We define an additive functor

$$
X \cdot-:_{\mathcal{O} G} \bmod \rightarrow_{A} \bmod , Y \mapsto X \cdot Y
$$

as follows: If $Y$ is an $\mathcal{O} G$-module and $\sigma: \mathcal{O} G \rightarrow \operatorname{End}_{\mathcal{O}}(Y)$ is the structural morphism, we denote by $X \cdot Y$ the $\mathcal{O}$-module $X \otimes_{\mathcal{O}} Y$. The action of $A$ on the latter is given by the morphism

$$
\rho \cdot \sigma: A \rightarrow \operatorname{End}_{\mathcal{O}}(X \otimes Y), \bar{a} a_{g} \mapsto \rho\left(\bar{a} a_{g}\right) \otimes \sigma(g) .
$$

Proposition 2.3.12 Let $X$ be an $\bar{A}$-module. The application

$$
A \otimes_{\bar{A}} X \rightarrow X \cdot \mathcal{O} G
$$

defined by

$$
a_{g} \otimes_{\bar{A}} x \mapsto \rho\left(a_{g}\right)(x) \otimes_{\mathcal{O}} g(\text { for all } x \in X \text { and } g \in G)
$$

is an isomorphism of $A$-modules

$$
\operatorname{Ind}_{\bar{A}}^{A}(X) \stackrel{\sim}{\rightarrow} X \cdot \mathcal{O} G
$$

\subsubsection{Induction and restriction of $K A$-modules and $K \bar{A}$ - modules}

Let $X$ be a $K A$-module of character $\chi$ and $Y$ a $K G$-module of character $\xi$. We denote by $\chi \cdot \xi$ the character of the $K A$-module $X \cdot Y$. From now on, all group algebras over $K$ will be considered split semisimple. 
Proposition 2.3.13 Let $\chi$ be an irreducible character of $K A$ which restricts to an irreducible character $\bar{\chi}$ of $K \bar{A}$. Then

1. The characters $(\chi \cdot \xi)_{\xi \in \operatorname{Irr}(K G)}$ are distinct irreducible characters of $K A$.

2. We have

$$
\operatorname{Ind}_{K \bar{A}}^{K A}(\bar{\chi})=\sum_{\xi \in \operatorname{Irr}(K G)} \xi(1)(\chi \cdot \xi)
$$

Proof: The second relation results from Proposition 2.3.12, We have

$$
\left\langle\operatorname{Ind}_{K \bar{A}}^{K A}(\bar{\chi}), \operatorname{Ind}_{K \bar{A}}^{K A}(\bar{\chi})\right\rangle_{K A}=\sum_{\xi, \xi^{\prime} \in \operatorname{Irr}(K G)} \xi(1) \xi^{\prime}(1)\left\langle\chi \cdot \xi, \chi \cdot \xi^{\prime}\right\rangle_{K A} .
$$

Frobenius reciprocity now gives

$$
\begin{aligned}
\left\langle\operatorname{Ind}_{K \bar{A}}^{K A}(\bar{\chi}), \operatorname{Ind}_{K \bar{A}}^{K A}(\bar{\chi})\right\rangle_{K A} & = & \left\langle\operatorname{Res}_{K \bar{A}}^{K A}\left(\sum_{\xi \in \operatorname{Irr}(K G)} \xi(1) \chi \cdot \xi\right), \bar{\chi}\right\rangle_{K \bar{A}} \\
& = & \left\langle\sum_{\xi \in \operatorname{Irr}(K G)} \xi(1)^{2} \bar{\chi}, \bar{\chi}\right\rangle_{K \bar{A}} \\
& = & \sum_{\xi \in \operatorname{Irr}(K G)} \xi(1)^{2}=|G|,
\end{aligned}
$$

whence we obtain

$$
|G|=\sum_{\xi, \xi^{\prime} \in \operatorname{Irr}(K G)} \xi(1) \xi^{\prime}(1)\left\langle\chi \cdot \xi, \chi \cdot \xi^{\prime}\right\rangle_{K A} .
$$

Since $|G|=\sum_{\xi \in \operatorname{Irr}(K G)} \xi(1)^{2}$, we must have $\left\langle\chi \cdot \xi, \chi \cdot \xi^{\prime}\right\rangle_{K A}=\delta_{\xi, \xi^{\prime}}$ and the proof is complete.

For all $\bar{\chi} \in \operatorname{Irr}(K \bar{A})$, we denote by $\bar{e}(\bar{\chi})$ the block of $K \bar{A}$ associated with $\bar{\chi}$. We have seen that if $g \in G$, then $g(\bar{e}(\bar{\chi}))$ is also a block of $K \bar{A}$. Since $K \bar{A}$ is split semisimple, it must be associated with an irreducible character $g(\bar{\chi})$ of $K \bar{A}$. Thus, we can define an action of $G$ on $\operatorname{Irr}(K \bar{A})$ such that for all $g \in G, \bar{e}(g(\bar{\chi}))=g(\bar{e}(\bar{\chi}))$. We denote by $G_{\bar{\chi}}$ the stabilizer of $\bar{\chi}$ in $G$. Obviously, $G_{\bar{\chi}}=G_{\bar{e}(\bar{\chi})}$.

Proposition 2.3.14 Let $\bar{\chi} \in \operatorname{Irr}(K \bar{A})$ and suppose that $\bar{\chi}$ is extended to a character $\tilde{\chi} \in \operatorname{Irr}\left(K A_{G_{\bar{\chi}}}\right)$ (i.e., $\left.\operatorname{Res}_{K \bar{A}}^{K A_{G_{\bar{\chi}}}}(\tilde{\chi})=\bar{\chi}\right)$. We set

$$
\chi:=\operatorname{Ind}_{K A_{G_{\bar{\chi}}}}^{K A}(\tilde{\chi}) \text { and } \chi_{\xi}:=\operatorname{Ind}_{K A_{G_{\bar{\chi}}}}^{K A}(\tilde{\chi} \cdot \xi) \text { for all } \xi \in \operatorname{Irr}\left(K G_{\bar{\chi}}\right) \text {. }
$$

Then

1. The characters $\left(\chi_{\xi}\right)_{\xi \in \operatorname{Irr}\left(K G_{\bar{\chi}}\right)}$ are distinct irreducible characters of $K A$. 
2. We have

$$
\operatorname{Ind}_{K \bar{A}}^{K A}(\bar{\chi})=\sum_{\xi \in \operatorname{Irr}\left(K G_{\bar{\chi}}\right)} \xi(1) \chi_{\xi}
$$

In particular,

$$
m_{\chi_{\xi}, \bar{\chi}}=\xi(1) \text { and } \chi_{\xi}(1)=\left|G: G_{\bar{\chi}}\right| \bar{\chi}(1) \xi(1) .
$$

3. For all $\xi \in \operatorname{Irr}\left(K G_{\bar{\chi}}\right)$, we have

$$
s_{\chi \xi}=\frac{\left|G_{\bar{\chi}}\right|}{\xi(1)} s_{\bar{\chi}} .
$$

\section{Proof:}

1. By Proposition 2.3 .13 , we obtain that the characters $(\tilde{\chi} \cdot \xi)_{\xi \in \operatorname{Irr}\left(K G_{\bar{\chi}}\right)}$ are distinct irreducible characters of $\operatorname{Irr}\left(K A_{G_{\bar{\chi}}}\right)$. Now let $\bar{e}(\bar{\chi})$ be the block of $K \bar{A}$ associated with the irreducible character $\bar{\chi}$. We have seen that $\bar{e}(\bar{\chi})$ is a central idempotent of $K A_{G_{\bar{\chi}}}$. Proposition 2.3 .11 implies that the functor $\operatorname{Ind}_{K \bar{A}}^{K A}$ defines a Morita equivalence between the category $K A_{G_{\bar{\chi}}} \bar{e}(\bar{\chi})$ mod and its image. Therefore, the characters $\left(\operatorname{Ind}_{K A_{G_{\bar{\chi}}}}^{K A}(\tilde{\chi} \cdot \xi)\right)_{\xi \in \operatorname{Irr}\left(K G_{\bar{\chi}}\right)}$ are distinct irreducible characters of $K A$.

2. By Proposition 2.3.13, we obtain that

$$
\operatorname{Ind}_{K \bar{A}}^{K A_{G_{\bar{\chi}}}}(\bar{\chi})=\sum_{\xi \in \operatorname{Irr}\left(K G_{\bar{\chi}}\right)} \xi(1) \tilde{\chi} \cdot \xi .
$$

Applying $\operatorname{Ind}_{K A_{G_{\bar{\chi}}}}^{K A}$ to both sides gives us the required relation. Obviously, $m_{\chi_{\xi}, \bar{\chi}}=\xi(1)$.

Now let us calculate the value of $\chi_{\xi}(\bar{a})$ for any $\bar{a} \in \bar{A}$. Let $Y$ be an irreducible $K A_{G_{\bar{\chi}}}$-module of character $\psi$. Then $\operatorname{Ind}_{K A_{G_{\bar{\chi}}}}^{K A}(Y)=K A \otimes_{K A_{G_{\bar{\chi}}}} Y$ has character $\operatorname{Ind}_{K A_{G_{\bar{\chi}}}}^{K A}(\psi)$. We have $K A=\bigoplus_{g \in G / G_{\bar{\chi}}} a_{g} K \bar{A}$. Let $\bar{a} \in \bar{A}$. Then

$$
\begin{aligned}
& \bar{a} \operatorname{Ind}_{K A_{G_{\bar{\chi}}}}^{K A}(Y)=\bigoplus_{g \in G / G_{\bar{\chi}}} \bar{a} a_{g} K \bar{A} \otimes_{K A_{G_{\bar{\chi}}}} Y \\
& =\bigoplus_{g \in G / G_{\bar{\chi}}} a_{g}\left(a_{g}^{-1} \bar{a} a_{g}\right) K \bar{A} \otimes_{K A_{G_{\bar{\chi}}}} Y \\
& =\bigoplus_{g \in G / G_{\bar{\chi}}} a_{g} K \bar{A} \otimes_{K A_{G_{\bar{\chi}}}}\left(a_{g}^{-1} \bar{a} a_{g}\right) Y \text {. }
\end{aligned}
$$

Thus, $\operatorname{Ind}_{K A_{G_{\bar{\chi}}}}^{K A}(\psi)(\bar{a})=\sum_{g \in G / G_{\bar{\chi}}} \psi\left(a_{g}^{-1} \bar{a} a_{g}\right)$ and

$$
\chi_{\xi}(\bar{a})=\sum_{g \in G / G_{\bar{\chi}}}(\tilde{\chi} \cdot \xi)\left(a_{g}^{-1} \bar{a} a_{g}\right)=\sum_{g \in G / G_{\bar{\chi}}} \bar{\chi}\left(a_{g}^{-1} \bar{a} a_{g}\right) \xi(1) .
$$


Therefore,

$$
\chi_{\xi}(1)=\sum_{g \in G / G_{\bar{\chi}}} \bar{\chi}(1) \xi(1)=\left|G: G_{\bar{\chi}}\right| \bar{\chi}(1) \xi(1) .
$$

3. Let $\left(\bar{e}_{i}\right)_{i \in I}$ be a basis of $\bar{A}$ as $\mathcal{O}$-module and let $\left(\bar{e}_{i}^{\prime}\right)_{i \in I}$ be its dual with respect to the symmetrizing form $\bar{t}$. Proposition $2.2 .12(3)$, in combination with Proposition 2.3.8, gives

$$
s_{\chi_{\xi}} \chi_{\xi}(1)^{2}=\chi_{\xi}\left(\sum_{i \in I, g \in G} \bar{e}_{i}^{\prime} a_{g} a_{g}^{-1} \bar{e}_{i}\right)=\chi_{\xi}\left(|G| \sum_{i \in I} \bar{e}_{i}^{\prime} \bar{e}_{i}\right) .
$$

However, $\sum_{i \in I, g \in G} \bar{e}_{i}^{\prime} a_{g} a_{g}^{-1} \bar{e}_{i}$ belongs to to center of $A$ (by Lemma 2.2.6) and thus, for all $h \in G$,

$$
a_{h}^{-1}\left(\sum_{i \in I} \bar{e}_{i}^{\prime} a_{g} a_{g}^{-1} \bar{e}_{i}\right) a_{h}=\sum_{i \in I} \bar{e}_{i}^{\prime} a_{g} a_{g}^{-1} \bar{e}_{i}=|G| \sum_{i \in I} \bar{e}_{i}^{\prime} \bar{e}_{i} .
$$

Since $\sum_{i \in I} \bar{e}_{i}^{\prime} \bar{e}_{i} \in \bar{A}$, we must have

$$
\begin{aligned}
\chi_{\xi}\left(|G| \sum_{i \in I} \bar{e}_{i}^{\prime} \bar{e}_{i}\right) & =\sum_{h \in G / G_{\bar{\chi}}} \bar{\chi}\left(a_{h}{ }^{-1}\left(|G| \sum_{i \in I} \bar{e}_{i}^{\prime} \bar{e}_{i}\right) a_{h}\right) \xi(1) \\
& =\sum_{h \in G / G_{\bar{\chi}}} \bar{\chi}\left(|G| \sum_{i \in I} \bar{e}_{i}^{\prime} \bar{e}_{i}\right) \xi(1) \\
& =\left|G: G_{\bar{\chi}}\right||G| \xi(1) \bar{\chi}\left(\sum_{i \in I} \bar{e}_{i}^{\prime} \bar{e}_{i}\right) \\
& =\left|G: G_{\bar{\chi}}\right|^{2}\left|G_{\bar{\chi}}\right| \xi(1) s_{\bar{\chi}} \bar{\chi}(1)^{2} .
\end{aligned}
$$

So we obtain

$$
s_{\chi_{\xi}} \chi_{\xi}(1)^{2}=\left|G: G_{\bar{\chi}}\right|^{2}\left|G_{\bar{\chi}}\right| \xi(1) \bar{\chi}(1)^{2} s_{\bar{\chi}} .
$$

Replacing $\chi_{\xi}(1)=\left|G: G_{\bar{\chi}}\right| \bar{\chi}(1) \xi(1)$ gives

$$
s_{\chi_{\xi}} \xi(1)=\left|G_{\bar{\chi}}\right| s_{\bar{\chi}} .
$$

Now let $\bar{\Omega}$ be the orbit of the character $\bar{\chi} \in \operatorname{Irr}(K \bar{A})$ under the action of $G$. We have $|\bar{\Omega}|=|G| /\left|G_{\bar{\chi}}\right|$. Define

$$
\bar{e}(\bar{\Omega})=\sum_{g \in G / G_{\bar{\chi}}} \bar{e}(g(\bar{\chi}))=\sum_{g \in G / G_{\bar{\chi}}} g(\bar{e}(\bar{\chi})) .
$$


If $\bar{\chi} \in \bar{\Omega}$, the set $\operatorname{Irr}(K A, \bar{\chi})$ depends only on $\bar{\Omega}$ and we $\operatorname{set} \operatorname{Irr}(K A, \bar{\Omega}):=$ $\operatorname{Irr}(K A, \bar{\chi})$. The idempotent $\bar{e}(\bar{\Omega})$ belongs to the algebra $(Z K \bar{A})^{G}$ of the elements in the center of $K \bar{A}$ fixed by $G$ and thus to the center of $K A$ (since it commutes with all elements of $\bar{A}$ and all $a_{g}, g \in G$ ). Therefore, it must be a sum of blocks of $K A$, i.e.,

$$
\bar{e}(\bar{\Omega})=\sum_{\chi \in \operatorname{Irr}(K A, \bar{\Omega})} e(\chi) .
$$

Let $X$ be an irreducible $K A$-module of character $\chi$ and $\bar{X}$ an irreducible $K \bar{A}$-submodule of $\operatorname{Res}_{K \bar{A}}^{K A}(X)$ of character $\bar{\chi}$. For $g \in G$, the $K \bar{A}$-submodule $g(\bar{X})$ of $\operatorname{Res}_{K \bar{A}}^{K A}(X)$ has character $g(\bar{\chi})$. Then $\sum_{g \in G} g(\bar{X})$ is a $K A$-submodule of $X$. We deduce that

$$
\operatorname{Res}_{K \bar{A}}^{K A}(X)=\left(\bigoplus_{g \in G / G_{\bar{\chi}}} g(\bar{X})\right)^{m_{\chi, \bar{\chi}}}
$$

i.e.,

$$
\operatorname{Res}_{K \bar{A}}^{K A}(\chi)=m_{\chi, \bar{\chi}} \sum_{g \in G / G_{\bar{\chi}}} g(\bar{\chi}) .
$$

In particular, we see that $\operatorname{Irr}(K \bar{A}, \chi)$ is an orbit of $G$ on $\operatorname{Irr}(K \bar{A})$. Notice that $\chi(1)=m_{\chi, \bar{\chi}}|\bar{\Omega}| \bar{\chi}(1)$.

Case where $G$ is cyclic

Suppose that $G$ is a cyclic group of order $d$ and let $g \in G$ be a generator of $G$ (we can choose $\operatorname{Rep}(A / \bar{A})=\left\{1, a_{g}, a_{g}^{2}, \ldots, a_{g}^{d-1}\right\}$ ). We will show that the assumptions of Proposition 2.3.14 are satisfied for all irreducible characters of $K \bar{A}$.

Let $\bar{X}$ be an irreducible $K \bar{A}$-module and let $\bar{\rho}: K \bar{A} \rightarrow \operatorname{End}_{K}(\bar{X})$ be the structural morphism. Since the representation of $\bar{X}$ is invariant by the action of $G_{\bar{\chi}}$, there exists an automorphism $\alpha$ of the $K$-vector space $\bar{X}$ such that

$$
\alpha \bar{\rho}(\bar{a}) \alpha^{-1}=g(\bar{\rho})(\bar{a}),
$$

for all $g \in G_{\bar{\chi}}$.

The subgroup $G_{\bar{\chi}}$ is also cyclic. Let $d(\bar{\chi}):=\left|G_{\bar{\chi}}\right|$. Then

$$
\bar{\rho}(\bar{a})=\alpha^{d(\bar{\chi})} \bar{\rho}(\bar{a}) \alpha^{-d(\bar{\chi})} .
$$

Since $\bar{X}$ is irreducible and $K \bar{A}$ is split semisimple, $\alpha^{d(\bar{\chi})}$ must be a scalar. Instead of enlarging the field $K$, we can assume that $K$ contains a $d(\bar{\chi})$-th 
root of that scalar. By dividing $\alpha$ by that root, we reduce to the case where $\alpha^{d(\bar{\chi})}=1$.

This allows us to extend the structural morphism $\bar{\rho}: K \bar{A} \rightarrow \operatorname{End}_{K}(\bar{X})$ to a morphism

$$
\tilde{\rho}: K A_{G_{\bar{\chi}}} \rightarrow \operatorname{End}_{K}(\bar{X})
$$

such that

$$
\tilde{\rho}\left(\bar{a} a_{h}^{j}\right):=\bar{\rho}(\bar{a}) \alpha^{j} \text { for } 0 \leq j<d(\bar{\chi}),
$$

where $h:=g^{d / d(\bar{\chi})}$ generates $G_{\bar{\chi}}$. The morphism $\tilde{\rho}$ defines a $K A_{G_{\bar{\chi}}}$-module $\tilde{X}$ of character $\tilde{\chi}$. By definition of $\tilde{\rho}, \operatorname{Res}_{K \bar{A}}^{K A_{G \bar{\chi}}}(\tilde{\chi})=\bar{\chi}$.

Since the group $G$ is abelian, the set $\operatorname{Irr}(K G)$ forms a group, which we denote by $G^{\vee}$. The application $\psi \mapsto \psi \cdot \xi$, where $\psi \in \operatorname{Irr}(K A)$ and $\xi \in G^{\vee}$, defines an action of $G^{\vee}$ on $\operatorname{Irr}(K A)$.

Let $\Omega$ be the orbit of $\tilde{\chi}$ under the action of $\left(G_{\bar{\chi}}\right)^{\vee}$. By Proposition 2.3.13, we obtain that $\Omega$ is a regular orbit (i.e., $|\Omega|=\left|G_{\bar{\chi}}\right|$ ) and that $\Omega=\operatorname{Irr}\left(K A_{G_{\bar{\chi}}}, \bar{\chi}\right)$. Like in Proposition 2.3.14, we introduce the notations

$$
\chi:=\operatorname{Ind}_{K A_{G_{\bar{\chi}}}}^{K A}(\tilde{\chi}) \text { and } \chi_{\xi}:=\operatorname{Ind}_{K A_{G_{\bar{\chi}}}}^{K A}(\tilde{\chi} \cdot \xi) \text { for all } \xi \in\left(G_{\bar{\chi}}\right)^{\vee} \text {. }
$$

Then

$$
\operatorname{Irr}(K A, \bar{\chi})=\left\{\chi_{\xi} \mid \xi \in\left(G_{\bar{\chi}}\right)^{\vee}\right\} \text { and } m_{\chi_{\xi}, \bar{\chi}}=\xi(1)=1 \text { for all } \xi \in\left(G_{\bar{\chi}}\right)^{\vee} .
$$

Recall that $\left|G_{\bar{\chi}}\right|=d(\bar{\chi})$. There exists a surjective morphism $G \rightarrow G_{\bar{\chi}}$ defined by $g \mapsto g^{d / d(\bar{\chi})}$, which induces an inclusion $\left(G_{\bar{\chi}}\right)^{\vee} \hookrightarrow G^{\vee}$. If $\xi \in$ $\left(G_{\bar{\chi}}\right)^{\vee}$, we denote (abusing notation) by $\xi$ its image in $G^{\vee}$ by the above injection. It is easy to check that $\chi_{\xi}=\chi \cdot \xi$.

Hence, Proposition 2.3.14 implies the following result.

Proposition 2.3.15 If the group $G$ is cyclic, then there exists a bijection

$$
\begin{array}{ccc}
\operatorname{Irr}(K \bar{A}) / G & \tilde{\leftrightarrow} & \operatorname{Irr}(K A) / G^{\vee} \\
\bar{\Omega} & \leftrightarrow & \Omega
\end{array}
$$

such that

$$
\bar{e}(\bar{\Omega})=e(\Omega),|\bar{\Omega}||\Omega|=|G| \text { and } \begin{cases}\forall \chi \in \Omega, & \operatorname{Res}_{K \bar{A}}^{K A}(\chi)=\sum_{\bar{\chi} \in \bar{\Omega}} \bar{\chi} \\ \forall \bar{\chi} \in \bar{\Omega}, & \operatorname{Ind}_{K \bar{A}}^{K A}(\bar{\chi})=\sum_{\chi \in \Omega} \chi\end{cases}
$$

Moreover, for all $\chi \in \Omega$ and $\bar{\chi} \in \bar{\Omega}$, we have

$$
s_{\chi}=|\Omega| s_{\bar{\chi}}
$$




\subsubsection{Blocks of $A$ and blocks of $\bar{A}$}

Let us denote by $\operatorname{Bl}(A)$ the set of blocks of $A$ and by $\operatorname{Bl}(\bar{A})$ the set of blocks of $\bar{A}$. For $\bar{b} \in \operatorname{Bl}(\bar{A})$, we have set

$$
\operatorname{Tr}(G, \bar{b}):=\sum_{g \in G / G_{\bar{b}}} g(\bar{b})
$$

The algebra $(Z \bar{A})^{G}$ is contained in both $Z \bar{A}$ and $Z A$ and the set of its blocks is

$$
\operatorname{Bl}\left((Z \bar{A})^{G}\right)=\{\operatorname{Tr}(G, \bar{b}) \mid \bar{b} \in \operatorname{Bl}(\bar{A}) / G\} .
$$

Moreover, $\operatorname{Tr}(G, \bar{b})$ is a sum of blocks of $A$ and we define the subset $\operatorname{Bl}(A, \bar{b})$ of $\operatorname{Bl}(A)$ as follows:

$$
\operatorname{Tr}(G, \bar{b}):=\sum_{b \in \operatorname{Bl}(A, \bar{b})} b
$$

Lemma 2.3.16 Let $\bar{b}$ be a block of $\bar{A}$ and $\bar{B}:=\operatorname{Irr}(K \bar{A} \bar{b})$. Then

1. For all $\bar{\chi} \in \bar{B}$, we have $G_{\bar{\chi}} \subseteq G_{\bar{b}}$.

2. We have

$$
\operatorname{Tr}(G, \bar{b})=\sum_{\bar{\chi} \in \bar{B} / G} \operatorname{Tr}(G, \bar{e}(\bar{\chi}))=\sum_{\{\bar{\Omega} \mid \bar{\Omega} \cap \bar{B} \neq \emptyset\}} \bar{e}(\bar{\Omega}) .
$$

\section{Proof:}

1. We have $\bar{b}=\sum_{\bar{\chi} \in \bar{B}} \bar{e}(\bar{\chi})$. If $g \notin G_{\bar{b}}$, then the blocks $\bar{b}$ and $g(\bar{b})$ are orthogonal. Hence, $g \notin G_{\bar{\chi}}$ for all $\bar{\chi} \in \bar{B}$.

2. Note that $\bar{b}=\sum_{\bar{\chi} \in \bar{B}} \bar{e}(\bar{\chi})=\sum_{\bar{\chi} \in \bar{B} / G_{\bar{b}}} \operatorname{Tr}\left(G_{\bar{b}}, \bar{e}(\bar{\chi})\right)$. Thus,

$$
\operatorname{Tr}(G, \bar{b})=\sum_{\bar{\chi} \in \bar{B} / G} \operatorname{Tr}(G, \bar{e}(\bar{\chi}))=\sum_{\{\bar{\Omega} \mid \bar{\Omega} \cap \bar{B} \neq \emptyset\}} \bar{e}(\bar{\Omega}),
$$

by the definition of $\bar{e}(\bar{\Omega})$.

Now let $G^{\vee}:=\operatorname{Hom}\left(G, K^{\times}\right)$. We suppose that $K=F$. The multiplication of the characters of $K A$ by the characters of $K G$ defines an action of the group $G^{\vee}$ on $\operatorname{Irr}(K A)$. This action is induced by the operation of $G^{\vee}$ on the algebra $A$, which is defined in the following way:

$$
\xi \cdot\left(\bar{a} a_{g}\right):=\xi(g) \bar{a} a_{g} \text { for all } \xi \in G^{\vee}, \bar{a} \in \bar{A}, g \in G .
$$


In particular, $G^{\vee}$ acts on the set of blocks of $A$. If $b$ is a block of $A$, we denote by $\xi \cdot b$ the product of $\xi$ and $b$ and by $\left(G^{\vee}\right)_{b}$ the stabilizer of $b$ in $G^{\vee}$. We set

$$
\operatorname{Tr}\left(G^{\vee}, b\right):=\sum_{\xi \in G^{\vee} /\left(G^{\vee}\right)_{b}} \xi \cdot b .
$$

The set of blocks of the algebra $(Z A)^{G^{\vee}}$ is given by

$$
\operatorname{Bl}\left((Z A)^{G^{\vee}}\right)=\left\{\operatorname{Tr}\left(G^{\vee}, b\right) \mid b \in \operatorname{Bl}(A) / G^{\vee}\right\} .
$$

The following lemma is the analogue of Lemma 2.3.16

Lemma 2.3.17 Let $b$ be $a$ block of $A$ and $B:=\operatorname{Irr}(K A b)$. Then

1. For all $\chi \in B$, we have $\left(G^{\vee}\right)_{\chi} \subseteq\left(G^{\vee}\right)_{b}$.

2. We have

$$
\operatorname{Tr}\left(G^{\vee}, b\right)=\sum_{\chi \in B / G^{\vee}} \operatorname{Tr}\left(G^{\vee}, e(\chi)\right)=\sum_{\{\Omega \mid \Omega \cap B \neq \emptyset\}} e(\Omega) .
$$

Case where $G$ is cyclic

For every orbit $\overline{\mathcal{Y}}$ of $G$ on $\operatorname{Bl}(\bar{A})$, we denote by $\bar{b}(\overline{\mathcal{Y}})$ the block of $(Z \bar{A})^{G}$ defined as

$$
\bar{b}(\overline{\mathcal{Y}}):=\sum_{\bar{b} \in \overline{\mathcal{Y}}} \bar{b}
$$

For every orbit $\mathcal{Y}$ of $G^{\vee}$ on $\operatorname{Bl}(A)$, we denote by $b(\mathcal{Y})$ the block of $(Z A)^{G^{\vee}}$ defined as

$$
b(\mathcal{Y}):=\sum_{b \in \mathcal{Y}} b
$$

The following proposition results from Proposition 2.3.15 and Lemmas 2.3 .16 and 2.3.17.

Proposition 2.3.18 If the group $G$ is cyclic, then there exists a bijection

$$
\begin{array}{ccc}
\operatorname{Bl}(\bar{A}) / G & \tilde{\leftrightarrow} & \operatorname{Bl}(A) / G^{\vee} \\
\overline{\mathcal{Y}} & \leftrightarrow & \mathcal{Y}
\end{array}
$$

such that

$$
\bar{b}(\overline{\mathcal{Y}})=b(\mathcal{Y})
$$

i.e.,

$$
\operatorname{Tr}(G, \bar{b})=\operatorname{Tr}\left(G^{\vee}, b\right) \text { for all } \bar{b} \in \overline{\mathcal{Y}} \text { and } b \in \mathcal{Y} .
$$

In particular, the algebras $(Z \bar{A})^{G}$ and $(Z A)^{G^{\vee}}$ have the same blocks.

Corollary 2.3.19 If the blocks of $A$ are stable under the action of $G^{\vee}$, then the blocks of $A$ coincide with the blocks of $(Z \bar{A})^{G}$. 


\subsection{Representation theory of symmetric al- gebras}

In the last section of Chapter 2, we present some results concerning the representation theory of symmetric algebras. If a symmetric algebra satisfies certain conditions, we can define a decomposition map (and consequently, a decomposition matrix) and obtain the blocks with the use of a Brauer graph. In order to check whether the required conditions are satisfied, we have to know when a symmetric algebra is split or semisimple. In subsection 2.4.4, we prove a theorem which gives us a new criterion for a symmetric algebra to be split and semisimple. With only this exception, all the results in this section are well-known and mostly taken from the seventh chapter of [33].

\subsubsection{Grothendieck groups}

Let $\mathcal{O}$ be an integral domain and $K$ a field containing $\mathcal{O}$. Let $A$ be an $\mathcal{O}$-algebra free and finitely generated as an $\mathcal{O}$-module.

Let $R_{0}(K A)$ be the Grothendieck group of finite-dimensional $K A$-modules. Thus, $R_{0}(K A)$ is generated by expressions $[V]$, one for each $K A$-module $V$ (up to isomorphism), with relations $[V]=\left[V^{\prime}\right]+\left[V^{\prime \prime}\right]$ for each exact sequence $0 \rightarrow V^{\prime} \rightarrow V \rightarrow V^{\prime \prime} \rightarrow 0$ of $K A$-modules. Two $K A$-modules $V, V^{\prime}$ give rise to the same element in $R_{0}(K A)$, if $V$ and $V^{\prime}$ have the same composition factors, counting multiplicities. It follows that $R_{0}(K A)$ is free abelian with basis given by the isomorphism classes of simple modules. Finally, let $R_{0}^{+}(K A)$ be the subset of $R_{0}(K A)$ consisting of elements $[V]$, where $V$ is a finite-dimensional $K A$-module.

Definition 2.4.1 Let $x$ be an indeterminate over $K$ and $\operatorname{Maps}(A, K[x])$ the $K$-algebra of maps from $A$ to $K[x]$ (with pointwise multiplication of maps as algebra multiplication). If $V$ is a $K A$-module, let $\rho_{V}: K A \rightarrow \operatorname{End}_{K}(V)$ denote its structural morphism. We define the map

$$
\begin{aligned}
\mathfrak{p}_{K}: R_{0}^{+}(K A) & \rightarrow \operatorname{Maps}(A, K[x]) \\
{[V] } & \mapsto\left(a \mapsto \text { characteristic polynomial of } \rho_{V}(a)\right) .
\end{aligned}
$$

Considering $\operatorname{Maps}(A, K[x])$ as a semigroup with respect to multiplication, the map $\mathfrak{p}_{K}$ is a semigroup homomorphism.

Let $\operatorname{Irr}(K A)$ be the set of all irreducible characters of the algebra $K A$ (i.e., the set of all characters $\chi_{V}$, where $V$ is a simple $K A$-module). The following result is known as the "Brauer-Nesbitt lemma" (cf. [15], Lemma $2)$. 
Lemma 2.4.2 Assume that $\operatorname{Irr}(K A)$ is a linearly independent subset of $\operatorname{Hom}_{K}(K A, K)$. Then the map $\mathfrak{p}_{K}$ is injective.

Proof: Let $V, V^{\prime}$ be two $K A$-modules such that $\mathfrak{p}_{K}([V])=\mathfrak{p}_{K}\left(\left[V^{\prime}\right]\right)$. Since $[V],\left[V^{\prime}\right]$ only depend on the composition factors of $V, V^{\prime}$, we may assume that $V, V^{\prime}$ are semisimple modules. Let

$$
V=\bigoplus_{i=1}^{n} a_{i} V_{i} \text { and } V^{\prime}=\bigoplus_{i=1}^{n} b_{i} V_{i},
$$

where the $V_{i}$ are pairwise non-isomorphic simple $K A$-modules and $a_{i}, b_{i} \geq 0$ for all $i$. We have to show that $a_{i}=b_{i}$ for all $i$.

If, for some $i$, we have both $a_{i}>0$ and $b_{i}>0$, then we can write $V=V_{i} \oplus \tilde{V}$ and $V^{\prime}=V_{i} \oplus \tilde{V}^{\prime}$. Since $\mathfrak{p}_{K}$ is a semigroup homomorphism, we obtain

$$
\mathfrak{p}_{K}\left(\left[V_{i}\right]\right) \cdot \mathfrak{p}_{K}([\tilde{V}])=\mathfrak{p}_{K}([V])=\mathfrak{p}_{K}\left(\left[V^{\prime}\right]\right)=\mathfrak{p}_{K}\left(\left[V_{i}\right]\right) \cdot \mathfrak{p}_{K}\left(\left[\tilde{V}^{\prime}\right]\right),
$$

and, dividing by $\mathfrak{p}_{K}\left(\left[V_{i}\right]\right)$, we conclude that $\mathfrak{p}_{K}([\tilde{V}])=\mathfrak{p}_{K}\left(\left[\tilde{V}^{\prime}\right]\right)$. Thus, we can suppose that, for all $i$, we have $a_{i}=0$ or $b_{i}=0$. Taking characters yields that

$$
\chi_{V}=\sum_{i} a_{i} \chi_{V_{i}} \text { and } \chi_{V^{\prime}}=\sum_{i} b_{i} \chi_{V_{i}} .
$$

For each $a \in A$, the character values $\chi_{V}(a)$ and $\chi_{V^{\prime}}(a)$ appear as coefficients in the polynomials $\mathfrak{p}_{K}([V])(a)$ and $\mathfrak{p}_{K}\left(\left[V^{\prime}\right]\right)(a)$ respectively. Since $\mathfrak{p}_{K}([V])=\mathfrak{p}_{K}\left(\left[V^{\prime}\right]\right)$, we deduce that $\sum_{i}\left(a_{i}-b_{i}\right) \chi_{V_{i}}=0$. By assumption, the characters $\chi_{V_{i}}$ are linearly independent. So we must have $\left(a_{i}-b_{i}\right) 1_{K}=0$ for all $i$. Since for all $i, a_{i}=0$ or $b_{i}=0$, this means that $a_{i} 1_{K}=0$ and $b_{i} 1_{K}=0$ for all $i$. If the field $K$ has characteristic 0 , we conclude that $a_{i}=b_{i}=0$ for all $i$ and we are done. If $K$ has characteristic $p>0$, we conclude that $p$ divides all $a_{i}$ and all $b_{i}$ and so $\frac{1}{p}[V]$ and $\frac{1}{p}\left[V^{\prime}\right]$ exist in $R_{0}^{+}(K A)$. We also have $\mathfrak{p}_{K}\left(\frac{1}{p}[V]\right)=\mathfrak{p}_{K}\left(\frac{1}{p}\left[V^{\prime}\right]\right)$. Repeating the above argument for $\frac{1}{p}[V]$ and $\frac{1}{p}\left[V^{\prime}\right]$ yields that the multiplicity of $V_{i}$ in each of these modules is still divisible by $p$. If we repeat this again and again, we deduce that $a_{i}$ and $b_{i}$ should be divisible by arbitrary powers of $p$. This forces $a_{i}=b_{i}=0$ for all $i$, as desired.

Remark: The assumption of the Brauer-Nesbitt lemma is satisfied when (but not only when) $K A$ is split or $K$ is a perfect field.

The following lemma (cf. [33, Lemma 7.3.4) implies the compatibility of the map $\mathfrak{p}_{K}$ with the field extensions of $K$. 
Lemma 2.4.3 Let $K \subseteq K^{\prime}$ be a field extension. Then there is a canonical map $d_{K}^{K^{\prime}}: R_{0}(K A) \rightarrow R_{0}\left(K^{\prime} A\right)$ given by $[V] \mapsto\left[K^{\prime} \otimes_{K} V\right]$. Furthermore, we have a commutative diagram

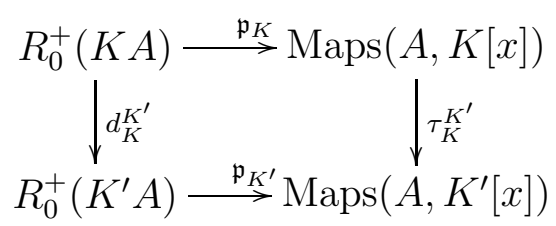

where $\tau_{K}^{K^{\prime}}$ is the canonical embedding. If, moreover, $K A$ is split, then $d_{K}^{K^{\prime}}$ is an isomorphism which preserves isomorphism classes of simple modules.

\subsubsection{Integrality}

We have seen in Chapter 1 that a subring $\mathcal{R} \subseteq K$ is a valuation ring if, for each non-zero element $x \in K$, we have $x \in \mathcal{R}$ or $x^{-1} \in \mathcal{R}$. Consequently, $K$ is the field of fractions of $\mathcal{R}$.

Such a valuation ring is a local ring whose maximal ideal we will denote by $\mathfrak{m}_{\mathcal{R}}$. Valuation rings have interesting properties, some of which are:

(V1) If $I$ is a prime ideal of $\mathcal{O}$, then there exists a valuation ring $\mathcal{R} \subseteq K$ such that $\mathcal{O} \subseteq \mathcal{R}$ and $\mathfrak{m}_{\mathcal{R}} \cap \mathcal{O}=I$.

(V2) Every finitely generated torsion-free module over a valuation ring in $K$ is free.

(V3) The intersection of all valuation rings $\mathcal{R} \subseteq K$ with $\mathcal{O} \subseteq \mathcal{R}$ is the integral closure of $\mathcal{O}$ in $K$; each valuation ring itself is integrally closed in $K$ (Proposition 1.2.15).

Lemma 2.4.4 Let $V$ be a $K A$-module. Choosing a $K$-basis of $V$, we obtain a corresponding matrix representation $\rho: K A \rightarrow \mathrm{M}_{n}(K)$, where $n=\operatorname{dim}_{K}(V)$. If $\mathcal{R} \subseteq K$ is a valuation ring with $\mathcal{O} \subseteq \mathcal{R}$, then a basis of $V$ can be chosen so that $\rho(a) \in \mathrm{M}_{n}(\mathcal{R})$ for all $a \in A$. In that case, we say that $V$ is realized over $\mathcal{R}$.

Proof: $\quad$ Let $\left(v_{1}, \ldots, v_{n}\right)$ be a $K$-basis of $V$ and $\mathcal{B}$ an $\mathcal{O}$-basis for $A$. Let $\tilde{V}$ be the $\mathcal{R}$-submodule of $V$ spanned by the finite set $\left\{v_{i} b \mid 1 \leq i \leq n, b \in \mathcal{B}\right\}$. Then $\tilde{V}$ is invariant under the action of $\mathcal{R} A$ and hence a finitely generated $\mathcal{R} A$-module. Since it is contained in a $K$-vector space, it is also torsion-free. So (V2) implies that $\tilde{V}$ is an $\mathcal{R} A$-lattice (a finitely generated $\mathcal{R} A$-module which is free as an $\mathcal{R}$-module) such that $K \otimes_{\mathcal{R}} \tilde{V} \cong V$. Thus, any $\mathcal{R}$-basis 
of $\tilde{V}$ is also a $K$-basis of $V$ with the required property.

Remark: Note that the above argument only requires that $\mathcal{R}$ is a subring of $K$ such that $K$ is the field of fractions of $\mathcal{R}$ and $\mathcal{R}$ satisfies (V2). These conditions also hold, for example, when $\mathcal{R}$ is a principal ideal domain with $K$ as field of fractions.

The following two important results ([33], Propositions 7.3.8 and 7.3.9) derive from the above lemma.

Proposition 2.4.5 Let $V$ be a $K A$-module and $\mathcal{O}_{K}$ be the integral closure of $\mathcal{O}$ in $K$. Then we have $\mathfrak{p}_{K}([V])(a) \in \mathcal{O}_{K}[x]$ for all $a \in A$. Thus the map $\mathfrak{p}_{K}$ of Definition 2.4.1 is in fact a map $R_{0}^{+}(K A) \rightarrow \operatorname{Maps}\left(A, \mathcal{O}_{K}[x]\right)$.

Proof: $\quad$ Fix $a \in A$. Let $\mathcal{R} \subseteq K$ be a valuation ring with $\mathcal{O} \subseteq \mathcal{R}$. By Lemma 2.4.4, there exists a basis of $V$ such that the action of $a$ on $V$ with respect to that basis is given by a matrix with coefficients in $\mathcal{R}$. Therefore, we have that $\mathfrak{p}_{K}([V])(a) \in \mathcal{R}[x]$. Since this holds for all valuation rings $\mathcal{R}$ in $K$ containing $\mathcal{O}$, property (V3) implies that $\mathfrak{p}_{K}([V])(a) \in \mathcal{O}_{K}[x]$.

Note that, in particular, Proposition 2.4.5 implies that $\chi_{V}(a) \in \mathcal{O}_{K}$ for all $a \in A$, where $\chi_{V}$ is the character of the representation $\rho_{V}$.

The next proposition is a result on symmetric algebras already mentioned in section 2.2 (Proposition 2.2.10): the integrality of the Schur elements.

Proposition 2.4.6 Assume that we have a symmetrizing form $t$ on $A$. Let $V$ be a split simple $K A$-module (i.e., $\operatorname{End}_{K A}(V) \cong K$ ) and let $s_{V}$ be its Schur element with respect to the induced form $t^{K}$ on $K A$. Then $s_{V} \in \mathcal{O}_{K}$.

Proof: $\quad$ Let $\mathcal{R} \subseteq K$ be a valuation ring with $\mathcal{O} \subseteq \mathcal{R}$. By Lemma 2.4.4, we can assume that $V$ affords a representation $\rho: K A \rightarrow \mathrm{M}_{n}(K)$ such that $\rho(a) \in \mathrm{M}_{n}(\mathcal{R})$ for all $a \in A$. Let $\mathcal{B}$ be an $\mathcal{O}$-basis of $A$ and let $\mathcal{B}^{\prime}$ be its dual with respect to $t$. Then $s_{V}=\sum_{b \in \mathcal{B}} \rho(b)_{i j} \rho\left(b^{\prime}\right)_{j i}$ for all $1 \leq i, j \leq n$ ([33], Cor. 7.2.2). All terms in the sum lie in $\mathcal{R}$ and so $s_{V} \in \mathcal{R}$. Since this holds for all valuation rings $\mathcal{R}$ in $K$ containing $\mathcal{O}$, property (V3) implies that $s_{V} \in \mathcal{O}_{K}$.

\subsubsection{The decomposition map}

Now, we moreover assume that the $\operatorname{ring} \mathcal{O}$ is integrally closed in $K$, i.e., $\mathcal{O}_{K}=\mathcal{O}$. Throughout we will fix a ring homomorphism $\theta: \mathcal{O} \rightarrow L$ into 
a field $L$ such that $L$ is the field of fractions of $\theta(\mathcal{O})$. We call such a ring homomorphism a specialization of $\mathcal{O}$.

Let $\mathcal{R} \subseteq K$ be a valuation ring with $\mathcal{O} \subseteq \mathcal{R}$ and $\mathfrak{m}_{\mathcal{R}} \cap \mathcal{O}=\operatorname{Ker} \theta$ (note that $\operatorname{Ker} \theta$ is a prime ideal, since $\theta(\mathcal{O})$ is contained in a field). Let $k$ be the residue field of $\mathcal{R}$. Then the restriction of the canonical map $\pi: \mathcal{R} \rightarrow k$ to $\mathcal{O}$ has kernel $\mathfrak{m}_{\mathcal{R}} \cap \mathcal{O}=\operatorname{Ker} \theta$. Since $L$ is the field of fractions of $\theta(\mathcal{O})$, we may regard $L$ as a subfield of $k$. Thus, we obtain a commutative diagram

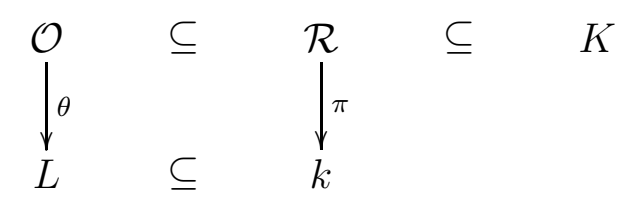

From now on, we make the following assumption:

Assumption 2.4.7 (a) $L A$ is split or (b) $L=k$ and $k$ is perfect.

The map $\theta: \mathcal{O} \rightarrow L$ induces a map $A \rightarrow L A, a \mapsto 1 \otimes a$. One consequence of the assumption 2.4.7 is that, due to Lemma 2.4.3, the map $d_{L}^{k}: R_{0}(L A) \rightarrow$ $R_{0}(k A)$ is an isomorphism which preserves isomorphism classes of simple modules. Thus we can identify $R_{0}(L A)$ and $R_{0}(k A)$. Moreover, the BrauerNesbitt lemma holds for $L A$, i.e., the map $\mathfrak{p}_{L}: R_{0}^{+}(L A) \rightarrow \operatorname{Maps}(A, L[x])$ is injective.

Let $V$ be a $K A$-module and $\mathcal{R} \subseteq K$ be a valuation ring with $\mathcal{O} \subseteq \mathcal{R}$. By Lemma 2.4.4, there exists a $K$-basis of $V$ such that the corresponding matrix representation $\rho: K A \rightarrow \mathrm{M}_{n}(K)\left(n=\operatorname{dim}_{K}(V)\right)$ has the property that $\rho(a) \in \mathrm{M}_{n}(\mathcal{R})$ for all $a \in A$. Following the proof of Lemma 2.4.4, that basis generates an $\mathcal{R} A$-lattice $\tilde{V}$ such that $K \otimes_{\mathcal{R}} \tilde{V}=V$. The $k$-vector space $k \otimes_{\mathcal{R}} \tilde{V}$ is a $k A$-module via $(1 \otimes v)(1 \otimes a)=1 \otimes v a(v \in \tilde{V}, a \in A)$, which we call the modular reduction of $\tilde{V}$. To simplify notation, we shall write

$$
K \tilde{V}:=K \otimes_{\mathcal{R}} \tilde{V} \text { and } k \tilde{V}:=k \otimes_{\mathcal{R}} \tilde{V} .
$$

The matrix representation $\rho^{k}: k A \rightarrow \mathrm{M}_{n}(k)$ afforded by $k \tilde{V}$ is given by

$$
\rho^{k}(1 \otimes a)=\left(\pi\left(a_{i j}\right)\right) \text { where } a \in A \text { and } \rho(a)=\left(a_{i j}\right) .
$$

Note that if $\tilde{V}^{\prime}$ is another $\mathcal{R} A$-lattice such that $K \otimes_{\mathcal{R}} \tilde{V}^{\prime} \cong V$, then $\tilde{V}$ and $\tilde{V}^{\prime}$ need not be isomorphic. The same hold for the $k A$-modules $k \otimes_{\mathcal{R}} \tilde{V}$ and $k \otimes_{\mathcal{R}} \tilde{V}^{\prime}$.

Now we are ready to state and prove the following result (cf. [33], Theorem 7.4.3), which associates to $A$ a decomposition map, in the case where $\mathcal{O}$ is integrally closed in $K$. 
Theorem-Definition 2.4.8 Let $\theta: \mathcal{O} \rightarrow L$ be a ring homomorphism into a field $L$ such that $L$ is the field of fractions of $\theta(\mathcal{O})$ and $\mathcal{O}$ is integrally closed in $K$. Assume that we have chosen a valuation ring $\mathcal{R}$ with $\mathcal{O} \subseteq \mathcal{R} \subseteq K$ and $\mathfrak{m}_{\mathcal{R}} \cap \mathcal{O}=\operatorname{Ker} \theta$ and that the assumption 2.4.7 is satisfied. Then

(a) The modular reduction induces an additive $\operatorname{map}_{\theta}: R_{0}^{+}(K A) \rightarrow R_{0}^{+}(L A)$ such that $d_{\theta}([K \tilde{V}])=[k \tilde{V}]$, where $\tilde{V}$ is an $\mathcal{R} A$-lattice and $[k \tilde{V}]$ is regarded as an element of $R_{0}^{+}(L A)$ via the identification of $R_{0}(k A)$ and $R_{0}(L A)$.

(b) By Proposition 2.4.5, the image of $\mathfrak{p}_{K}$ is contained in $\operatorname{Maps}(A, \mathcal{O}[x])$ and we have the following commutative diagram

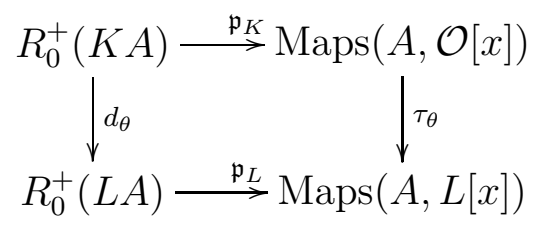

where $\tau_{\theta}: \operatorname{Maps}(A, \mathcal{O}[x]) \rightarrow \operatorname{Maps}(A, L[x])$ is the map induced by $\theta$.

(c) The map $d_{\theta}$ is uniquely determined by the commutativity of the above diagram. In particular, the map $d_{\theta}$ depends only on $\theta$ and not on the choice of $\mathcal{R}$.

The map $d_{\theta}$ will be called the decomposition map associated with the specialization $\theta: \mathcal{O} \rightarrow L$. The matrix of that map with respect to the bases of $R_{0}(K A)$ and $R_{0}(L A)$ consisting of the classes of the simple modules is called the decomposition matrix associated with $\theta$.

Proof: $\quad$ Let $\tilde{V}$ be an $\mathcal{R} A$-lattice and $a \in A$. Let $\left(a_{i j}\right) \in \mathrm{M}_{n}(\mathcal{R})$ be the matrix describing the action of $a$ on $\tilde{V}$ with respect to a chosen $\mathcal{R}$-basis of $\tilde{V}$. Due to the properties of modular reduction, the action of $1 \otimes a \in k A$ on $k \tilde{V}$ is given by the matrix $\left(\pi\left(a_{i j}\right)\right)$. Then, by definition, $\mathfrak{p}_{L}([k \tilde{V}])(a)$ is the characteristic polynomial of $\left(\pi\left(a_{i j}\right)\right)$. On the other hand, applying $\theta$ (which is the restriction of $\pi$ to $\mathcal{O}$ ) to the coefficients of the characteristic polynomial of $\left(a_{i j}\right)$ returns $\left(\tau_{\theta} \circ \mathfrak{p}_{K}\right)([K \tilde{V}])(a)$. Since the two actions just described commute, the two polynomials obtained are equal. Thus the following relation is established:

$$
\mathfrak{p}_{L}([k \tilde{V}])=\tau_{\theta} \circ \mathfrak{p}_{K}([K \tilde{V}]) \text { for all } \mathcal{R} A \text {-lattices } \tilde{V}
$$

Now let us prove $(a)$. We have to show that the map $d_{\theta}$ is well-defined i.e., if $\tilde{V}, \tilde{V}^{\prime}$ are two $\mathcal{R} A$-lattices such that $K \tilde{V}$ and $K \tilde{V}^{\prime}$ have the same composition factors (counting multiplicities), then the classes of $k \tilde{V}$ and $k \tilde{V}^{\prime}$ 
in $R_{0}(L A)$ are the same. Since $[K \tilde{V}]=\left[K \tilde{V}^{\prime}\right]$, the endomorphisms $\rho_{K \tilde{V}}(a)$ and $\rho_{K \tilde{V}^{\prime}}(a)$ are conjugate for all $a \in A$. The equality $(\dagger)$ implies that

$$
\mathfrak{p}_{L}([k \tilde{V}])(a)=\mathfrak{p}_{L}\left(\left[k \tilde{V}^{\prime}\right]\right)(a) \text { for all } a \in A .
$$

We have already remarked that, since the assumption 2.4 .7 is satisfied, the Brauer-Nesbitt lemma holds for $L A$. So we conclude that $[k \tilde{V}]=\left[k \tilde{V}^{\prime}\right]$, as desired.

Having established the existence of $d_{\theta}$, we have $[k \tilde{V}]=d_{\theta}([K \tilde{V}])$ for any $\mathcal{R} A$-lattice $\tilde{V}$. Hence $(\dagger)$ yields the commutativity of the diagram in $(b)$.

Finally, by the Brauer-Nesbitt lemma, the map $\mathfrak{p}_{L}$ is injective. Hence there exists at most one map which makes the diagram in (b) commutative. This proves (c).

Remark: Note that if $\mathcal{O}$ is a discrete valuation ring and $L$ its residue field, we do not need the assumption 2.4.7 in order to define a decomposition map from $R_{0}^{+}(K A)$ to $R_{0}^{+}(L A)$ associated with the canonical map $\theta: \mathcal{O} \rightarrow L$. For a given $K A$-module $V$, there exists an $A$-lattice $\tilde{V}$ such that $V=K \otimes_{\mathcal{O}} \tilde{V}$. The map $d_{\theta}: R_{0}^{+}(K A) \rightarrow R_{0}^{+}(L A),[K \tilde{V}] \mapsto[L \tilde{V}]$ is well and uniquely defined. For the details of this construction, see [27], $\$ 16 \mathrm{C}$.

Recall from Proposition 2.4.5 that if $V$ is a $K A$-module, then its character $\chi_{V}$ restricts to a trace function $\dot{\chi}_{V}: A \rightarrow \mathcal{O}$. Now, any linear map $\lambda: A \rightarrow \mathcal{O}$ induces an $L$-linear map

$$
\lambda^{L}: L A \rightarrow L, 1 \otimes a \mapsto \theta(\lambda(a))(a \in A) .
$$

It is clear that if $\lambda$ is a trace function, so is $\lambda^{L}$. Applying this to $\dot{\chi}_{V}$ shows that $\dot{\chi}_{V}^{L}$ is a trace function on $L A$. Since character values occur as coefficients in characteristic polynomials, Theorem 2.4 .8 implies that $\dot{\chi}_{V}^{L}$ is the character of $d_{\theta}([V])$. Moreover, for any simple $K A$-module $V$, we have

$$
\dot{\chi}_{V}^{L}=\sum_{V^{\prime}} d_{V V^{\prime}} \chi_{V^{\prime}}
$$

where the sum is over all simple $L A$-modules $V^{\prime}$ (up to isomorphism) and $D=\left(d_{V V^{\prime}}\right)$ is the decomposition matrix associated with $\theta$.

The following result gives a criterion for $d_{\theta}$ to be trivial. It is known as "Tits' deformation theorem". For its proof, the reader may refer, for example, to [33], Theorem 7.4.6.

Theorem 2.4.9 Assume that $K A$ and $L A$ are split. If $L A$ is semisimple, then $K A$ is also semisimple and the decomposition map $d_{\theta}$ is an isomorphism which preserves isomorphism classes of simple modules. In particular, the map $\operatorname{Irr}(K A) \rightarrow \operatorname{Irr}(L A), \chi \mapsto \dot{\chi}^{L}$ is a bijection. 
Finally, if $A$ is symmetric, we can check whether the assumption of Tits' deformation theorem is satisfied, using the following semisimplicity criterion (cf. [33], Thm. 7.4.7).

Theorem 2.4.10 Assume that $K A$ and $L A$ are split and that $A$ is symmetric with symmetrizing form $t$. For any simple $K A$-module $V$, let $s_{V} \in \mathcal{O}$ be the Schur element with respect to the induced symmetrizing form $t^{K}$ on $K A$. Then $L A$ is semisimple if and only if $\theta\left(s_{V}\right) \neq 0$ for all $V$.

Corollary 2.4.11 Let $K$ be the field of fractions of $\mathcal{O}$. Assume that $K A$ is split semisimple and that $A$ is symmetric with symmetrizing form $t$. If the map $\theta$ is injective, then $L A$ is split semisimple.

\subsubsection{A variation for Tits' deformation theorem}

Let us suppose that $\mathcal{O}$ is a Krull ring and $\theta: \mathcal{O} \rightarrow L$ is a ring homomorphism into a field $L$ such that $L$ is the field of fractions of $\theta(\mathcal{O})$. We will give a new criterion for the algebra $L A$ to be split semisimple.

Theorem 2.4.12 Let $K$ be the field of fractions of $\mathcal{O}$. Assume that $K A$ is split semisimple and that $A$ is symmetric with symmetrizing form $t$. For any simple $K A$-module $V$, let $s_{V} \in \mathcal{O}$ be the Schur element with respect to the induced symmetrizing form $t^{K}$ on $K A$. If $\operatorname{Ker} \theta$ is a prime ideal of $\mathcal{O}$ of height 1 , then $L A$ is split semisimple if and only if $\theta\left(s_{V}\right) \neq 0$ for all $V$.

Proof: $\quad$ If $L A$ is split semisimple, then Theorem 2.4.10 implies that $\theta\left(s_{V}\right) \neq$ 0 for all $V$. Now let us denote by $\operatorname{Irr}(K A)$ the set of irreducible characters of $K A$. If $\chi$ is the character afforded by a simple $K A$-module $V_{\chi}$, then $s_{\chi}:=s_{V_{\chi}}$. We set $\mathfrak{q}:=\operatorname{Ker} \theta$ and suppose that $s_{\chi} \notin \mathfrak{q}$ for all $\chi \in \operatorname{Irr}(K A)$. Since $K A$ is split semisimple, it is isomorphic to a product of matrix algebras over $K$ :

$$
K A \cong \prod_{\chi \in \operatorname{Irr}(K A)} \operatorname{End}_{K}\left(V_{\chi}\right)
$$

Let us denote by $\pi_{\chi}: K A \rightarrow \operatorname{End}_{K}\left(V_{\chi}\right)$ the projection onto the $\chi$-factor, such that $\pi:=\prod_{\chi \in \operatorname{Irr}(K A)} \pi_{\chi}$ is the above isomorphism. Then $\chi=\operatorname{tr}_{V_{\chi}} \circ \pi_{\chi}$, where $\operatorname{tr}_{V_{\chi}}$ denotes the standard trace on $\operatorname{End}_{K}\left(V_{\chi}\right)$.

Let $\mathcal{B}, \mathcal{B}^{\prime}$ be two dual bases of $A$ with respect to the symmetrizing form t. By Lemma 2.2.7, for all $a \in K A$ and $\chi \in \operatorname{Irr}(K A)$, we have

$$
\chi^{\vee} a=\sum_{b \in \mathcal{B}} \chi\left(b^{\prime} a\right) b .
$$


Applying $\pi$ to both sides yields

$$
\pi\left(\chi^{\vee}\right) \pi(a)=\sum_{b \in \mathcal{B}} \chi\left(b^{\prime} a\right) \pi(b) .
$$

By definition of the Schur element, if $\omega_{\chi}$ denotes the central character associated with $\chi$, then $s_{\chi}=\omega_{\chi}\left(\chi^{\vee}\right)=\pi_{\chi}\left(\chi^{\vee}\right)=\pi\left(\chi^{\vee}\right)$. Thus, if $\alpha \in \operatorname{End}_{K}\left(V_{\chi}\right)$, then

$$
\pi^{-1}(\alpha)=\frac{1}{s_{\chi}} \sum_{b \in \mathcal{B}} \operatorname{tr}_{V_{\chi}}\left(\pi_{\chi}\left(b^{\prime}\right) \alpha\right) b .
$$

Since $\mathcal{O}$ is a Krull ring and $\mathfrak{q}$ is a prime ideal of height 1 of $\mathcal{O}$, the ring $\mathcal{O}_{\mathfrak{q}}$ is, by Theorem 1.2.24, a discrete valuation ring. Thanks to Lemma 2.4.4, there exists an $\mathcal{O}_{\mathfrak{q}} A$-lattice $\tilde{V}_{\chi}$ such that $K \otimes_{\mathcal{O}_{\mathfrak{q}}} \tilde{V}_{\chi} \cong V_{\chi}$.

Moreover, $1 / s_{\chi} \in \mathcal{O}_{\mathfrak{q}}$ for all $\chi \in \operatorname{Irr}(K A)$. Due to the relation $(\dagger)$, the map $\pi$ induces an isomorphism

$$
\mathcal{O}_{\mathfrak{q}} A \cong \prod_{\chi \in \operatorname{Irr}(K A)} \operatorname{End}_{\mathcal{O}_{\mathfrak{q}}}\left(\tilde{V}_{\chi}\right)
$$

i.e., $\mathcal{O}_{\mathfrak{q}} A$ is isomorphic to a product of matrix algebras over $\mathcal{O}_{\mathfrak{q}}$. Since $\operatorname{Ker} \theta=\mathfrak{q}$, the above isomorphism remains after applying $\theta$ to both sides. Therefore, we obtain that $L A$ is isomorphic to a product of matrix algebras over $L$ and thus split semisimple.

If that is the case, then the assumption of Tits' deformation theorem is satisfied and there exists a bijection $\operatorname{Irr}(K A) \leftrightarrow \operatorname{Irr}(L A)$.

\subsubsection{Symmetric algebras over discrete valuation rings}

From now on, we assume that the following conditions are satisfied:

- $\mathcal{O}$ is a discrete valuation ring in $K$ and $K$ is perfect; let $v: K \rightarrow \mathbb{Z} \cup\{\infty\}$ be the corresponding valuation and $\mathfrak{p}$ the maximal ideal of $\mathcal{O}$.

- $K A$ is split semisimple.

- $\theta: \mathcal{O} \rightarrow L$ is the canonical map onto the residue field $L$ of $\mathcal{O}$.

- $A$ is a symmetric algebra with symmetrizing form $t$.

We have already seen that we have a well-defined decomposition map $d_{\theta}: R_{0}^{+}(K A) \rightarrow R_{0}^{+}(L A)$. The decomposition matrix associated with $d_{\theta}$ is 
the $|\operatorname{Irr}(K A)| \times|\operatorname{Irr}(L A)|$ matrix $D=\left(d_{\chi \phi}\right)$ with non-negative integer entries such that

$$
d_{\theta}\left(\left[V_{\chi}\right]\right)=\sum_{\phi \in \operatorname{Irr}(L A)} d_{\chi \phi}\left[V_{\phi}^{\prime}\right] \text { for all } \chi \in \operatorname{Irr}(K A),
$$

where $V_{\chi}$ is a simple $K A$-module with character $\chi$ and $V_{\phi}^{\prime}$ is a simple $L A$ module with character $\phi$. We sometimes call the characters of $K A$ "ordinary" and the characters of $L A$ "modular". We say that $\phi \in \operatorname{Irr}(L A)$ is a modular constituent of $\chi \in \operatorname{Irr}(K A)$, if $d_{\chi \phi} \neq 0$.

The rows of $D$ describe the decomposition of $d_{\theta}\left(\left[V_{\chi}\right]\right)$ in the standard basis of $R_{0}(L A)$. An interpretation of the columns is given by the following result (cf. [33], Theorem 7.5.2), which is part of Brauer's classical theory of modular representations ("Brauer reciprocity").

Theorem 2.4.13 For each $\phi \in \operatorname{Irr}(L A)$, there exists some primitive idempotent $e_{\phi} \in A$ such that

$$
\left[e_{\phi} K A\right]=\sum_{\chi \in \operatorname{Irr}(K A)} d_{\chi \phi}\left[V_{\chi}\right] \in R_{0}^{+}(K A)
$$

Let $\phi \in \operatorname{Irr}(L A)$. Consider the map $\psi(\phi): Z K A \rightarrow K$ defined by

$$
\psi(\phi):=\sum_{\chi \in \operatorname{Irr}(K A)} \frac{d_{\chi \phi}}{s_{\chi}} \omega_{\chi},
$$

where $\omega_{\chi}: Z K A \rightarrow K$ is the central character associated with $\chi \in \operatorname{Irr}(K A)$, as defined in subsection 2.1.4. The next result is due to Geck and Rouquier (cf. 34, Proposition 4.4).

Theorem 2.4.14 The map $\psi(\phi)$ restricts to a map $Z A \rightarrow \mathcal{O}$. In particular,

$$
\psi(\phi)(1)=\sum_{\chi \in \operatorname{Irr}(K A)} \frac{d_{\chi \phi}}{s_{\chi}} \in \mathcal{O} .
$$

Proof: Let us denote by $t^{K}$ the induced symmetrizing form on $K A$. If $e_{\phi}$ is an idempotent as in Theorem 2.4.13, then we can define a $K$-linear map $\lambda_{\phi}: Z K A \rightarrow K, z \mapsto t^{K}\left(z e_{\phi}\right)$. We claim that $\lambda_{\phi}=\psi(\phi)$. Since $K A$ is split semisimple, the elements $\left\{\chi^{\vee} \mid \chi \in \operatorname{Irr}(K A)\right\}$ form a basis of $Z K A$ (recall that $\chi^{\vee}$ is the element of $Z K A$ such that $t^{K}\left(\chi^{\vee} x\right)=\chi(x)$ for all $\left.x \in K A\right)$. It is, therefore, sufficient to show that

$$
\lambda_{\phi}\left(\chi^{\vee}\right)=\psi(\phi)\left(\chi^{\vee}\right) \text { for all } \chi \in \operatorname{Irr}(K A) .
$$


We have $\psi(\phi)\left(\chi^{\vee}\right)=d_{\chi \phi} 1_{K}$. Now consider the left-hand side.

$$
\begin{aligned}
\lambda_{\phi}\left(\chi^{\vee}\right) & =t^{K}\left(\chi^{\vee} e_{\phi}\right)=\chi\left(e_{\phi}\right)=\operatorname{dim}_{K}\left(V_{\chi} e_{\phi}\right) 1_{K} \\
& =\operatorname{dim}_{K}\left(\operatorname{Hom}_{K}\left(e_{\phi} K A, V_{\chi}\right)\right) 1_{K}=d_{\chi \phi} 1_{K} .
\end{aligned}
$$

Hence the above claim is established.

Finally, it remains to observe that since $e_{\phi} \in A$, the function $\lambda_{\phi}$ takes values in $\mathcal{O}$ on all elements of $A$.

Finally, we will treat the block distribution of characters. For this purpose, we introduce the following notions.

\section{Definition 2.4.15}

1. The Brauer graph associated with $A$ has vertices labeled by the irreducible characters of $K A$ and an edge joining $\chi, \chi^{\prime} \in \operatorname{Irr}(K A)$ if $\chi \neq \chi^{\prime}$ and there exists some $\phi \in \operatorname{Irr}(L A)$ such that $d_{\chi \phi} \neq 0 \neq d_{\chi^{\prime} \phi}$, i.e., there. A connected component of the Brauer graph is called a block.

2. Let $\chi \in \operatorname{Irr}(K A)$. Recall that $0 \neq s_{\chi} \in \mathcal{O}$. Let $\delta_{\chi}:=v\left(s_{\chi}\right)$, where $v$ is the given valuation. Then $\delta_{\chi}$ is called the defect of $\chi$ and we have $\delta_{\chi} \geq 0$ for all $\chi \in \operatorname{Irr}(K A)$. If $B$ is a block, then $\delta_{B}:=\max \left\{\delta_{\chi} \mid \chi \in B\right\}$ is called the defect of $B$.

Following [31, I.17.9, each block $B$ of $A$ corresponds to a central primitive idempotent (i.e., block-idempotent) $e_{B}$ of $A$. If $\chi \in B$ and $e_{\chi}$ is its corresponding central primitive idempotent in $K A$, then $e_{B} e_{\chi} \neq 0$.

Every $\chi \in \operatorname{Irr}(K A)$ determines a central character $\omega_{\chi}: Z K A \rightarrow K$. Since $\mathcal{O}$ is integrally closed, we have $\omega_{\chi}(z) \in \mathcal{O}$ for all $z \in Z A$. We have the following standard result (already presented in subsection 2.1.4) relating blocks with central characters.

Proposition 2.4.16 Let $\chi, \chi^{\prime} \in \operatorname{Irr}(K A)$. Then $\chi$ and $\chi^{\prime}$ belong to the same block of $A$ if and only if

$$
\theta\left(\omega_{\chi}(z)\right)=\theta\left(\omega_{\chi^{\prime}}(z)\right) \text { for all } z \in Z A \text {. }
$$

i.e.,

$$
\omega_{\chi}(z) \equiv \omega_{\chi^{\prime}}(z) \bmod \mathfrak{p} \text { for all } z \in Z A \text {. }
$$


Proof: First assume that $\chi, \chi^{\prime}$ belong to the same block of $A$, i.e., they belong to a connected component of the Brauer graph. It is sufficient to consider the case where $\chi, \chi^{\prime}$ are directly linked on the Brauer graph, i.e., there exists some $\phi \in \operatorname{Irr}(L A)$ such that $d_{\chi \phi} \neq 0 \neq d_{\chi^{\prime} \phi}$. Let $\tilde{V}_{\chi}$ (resp. $\tilde{V}_{\chi^{\prime}}$ ) be an $A$-lattice such that $K \tilde{V}_{\chi}$ (resp. $\left.K \tilde{V}_{\chi^{\prime}}\right)$ affords $\chi$ (resp. $\left.\chi^{\prime}\right)$. Let $z \in Z A$. Then $1 \otimes z$ acts by the scalar $\theta\left(\omega_{\chi}(z)\right)$ on every modular constituent of $k \tilde{V}_{\chi}$. Similarly, $1 \otimes z$ acts by the scalar $\theta\left(\omega_{\chi^{\prime}}(z)\right)$ on every modular constituent of $k \tilde{V}_{\chi^{\prime}}$. Since, by assumption, $K \tilde{V}_{\chi}$ and $K \tilde{V}_{\chi^{\prime}}$ have a modular constituent in common, we have $\theta\left(\omega_{\chi}(z)\right)=\theta\left(\omega_{\chi^{\prime}}(z)\right)$, as desired.

Now assume that $\chi$ belongs to the block $B$ and $\chi^{\prime}$ to the block $B^{\prime}$, with $B \neq B^{\prime}$. Let $e_{B}, e_{B^{\prime}}$ be the corresponding central primitive idempotents. Then $\omega_{\chi}\left(e_{B}\right)=1$ and $\omega_{\chi^{\prime}}\left(e_{B}\right)=0$. Consequently, $\theta\left(\omega_{\chi}\left(e_{B}\right)\right) \neq \theta\left(\omega_{\chi^{\prime}}\left(e_{B}\right)\right)$.

Using the above characterization of blocks, we can prove the following result about the characters of defect 0 , i.e., the characters whose Schur elements do not belong to the maximal ideal $\mathfrak{p}$ of $\mathcal{O}$.

Theorem 2.4.17 Let $\chi \in \operatorname{Irr}(K A)$ with $\theta\left(s_{\chi}\right) \neq 0$. Then $\chi$ is an isolated vertex in the Brauer graph and the corresponding decomposition matrix is just (1).

Proof: Let $t^{K}$ be the induced symmetrizing form on $K A$ and $\hat{t}^{K}$ the isomorphism from $K A$ to $\operatorname{Hom}_{K}(K A, K)$ induced by $t^{K}$. The irreducible character $\chi \in \operatorname{Irr}(K A)$ is a trace function on $K A$ and thus we can define $\chi^{\vee}:=\left(\hat{t}^{K}\right)^{-1}(\chi) \in Z K A$. Since $\chi$ restricts to a trace function $A \rightarrow \mathcal{O}$, we have in fact $\chi^{\vee} \in Z A$. By definition, we have that $\omega_{\chi}\left(\chi^{\vee}\right)=s_{\chi}$ and $\omega_{\chi^{\prime}}\left(\chi^{\vee}\right)=0$ for any $\chi^{\prime} \in \operatorname{Irr}(K A), \chi^{\prime} \neq \chi$. Now assume that there exists some character $\chi^{\prime}$ which is linked to $\chi$ in the Brauer graph. Proposition 2.4.16 implies that $0 \neq \theta\left(s_{\chi}\right)=\theta\left(\omega_{\chi}\left(\chi^{\vee}\right)\right)=\theta\left(\omega_{\chi^{\prime}}\left(\chi^{\vee}\right)\right)=0$, which is absurd.

It remains to show that $d_{\theta}\left(\left[V_{\chi}\right]\right)$ is the class of a simple module in $R_{0}^{+}(L A)$. By Lemma 2.4.4, there exists a basis of $V_{\chi}$ and a corresponding representation $\rho: K A \rightarrow \mathrm{M}_{n}(K)$ afforded by $V_{\chi}$ such that $\rho(a) \in \mathrm{M}_{n}(\mathcal{O})$ for all $a \in A$. Let $\mathcal{B}$ be an $\mathcal{O}$-basis of $A$ and let $\mathcal{B}^{\prime}$ be its dual with respect to $t$. We have seen in the proof of Proposition 2.4.6 that $s_{\chi}=\sum_{b \in \mathcal{B}} \rho(b)_{i j} \rho\left(b^{\prime}\right)_{j i}$ for all $1 \leq i, j \leq n$. All terms in this expression lie in $\mathcal{O}$. So we can apply the map $\theta$ and obtain a similar relation for $\theta\left(s_{\chi}\right)$ with respect to the module $L \tilde{V}_{\chi}$, where $\tilde{V}_{\chi} \subseteq V_{\chi}$ is the $A$-lattice spanned by the above basis of $V_{\chi}$. Since $\theta\left(s_{\chi}\right) \neq 0$, the module $L \tilde{V}_{\chi}$ is simple ([33], Lemma 7.2.3).

The next result ([52], Lemma 2.6(b)) is a consequence of Theorems 2.4.14 and 2.4.17. 
Proposition 2.4.18 Assume that the canonical map $Z A \rightarrow Z L A$ is surjective and let $\chi \in \operatorname{Irr}(K A)$. Then $\chi$ is a block by itself if and only if $\theta\left(s_{\chi}\right) \neq 0$. 


\section{Chapter 3}

\section{On Essential algebras}

In this chapter we introduce the notion of "essential algebras". These are symmetric algebras defined over a Laurent polynomial ring whose Schur elements are polynomials of a specific form (described by Definition 3.1.1). This form gives rise to the definition of the "essential monomials" for the algebra. As we have seen in the previous chapter, the Schur elements play an important role in the determination of the blocks of a symmetric algebra. In the following sections, we see how the form of the Schur elements affects the behavior of the blocks of an essential algebra when specialized via different types of morphisms (a morphism associated with a monomial in 3.2 , an adapted morphism in 3.3, the morphism $I^{n}$ defined in 3.4). In particular, in the first two cases, we show that the blocks depend only on the essential monomials for the algebra.

In the next chapter, we will see that the generic Hecke algebras of complex reflection groups are a particular case of essential algebras.

\subsection{Generalities}

Let $R$ be a Noetherian integrally closed domain with field of fractions $K$. Let $\mathbf{x}=\left(x_{i}\right)_{0 \leq i \leq m}$ be a set of $m+1$ indeterminates over $R$. We set $A:=R\left[\mathbf{x}, \mathbf{x}^{-1}\right]$ the Laurent polynomial ring in these indeterminates. The ring $A$ is also a Noetherian integrally closed domain and thus a Krull ring, by Proposition 1.2.26. The field of fractions of $A$ is $K(\mathbf{x})$. Let $H$ be an $A$-algebra such that

- $H$ is free and finitely generated as an $A$-module.

- There exists a linear form $t: H \rightarrow A$ which is symmetrizing on $H$.

- The algebra $K(\mathbf{x}) H:=K(\mathbf{x}) \otimes_{A} H$ is split semisimple. 
Due to Proposition 2.2.12, we have that the symmetrizing form $t$ is of the form

$$
t=\sum_{\chi \in \operatorname{Irr}(K(\mathbf{x}) H)} \frac{1}{s_{\chi}} \chi,
$$

where $s_{\chi}$ denotes the Schur element of $\chi$ with respect to $t$. We know that $s_{\chi} \in A$ by Proposition 2.2.10. Moreover, for all $\chi \in \operatorname{Irr}(K(\mathbf{x}) H)$, the blockidempotent of $K(\mathbf{x}) H$ associated to $\chi$ is $e_{\chi}=\chi^{\vee} / s_{\chi}$ (for the definition and properties of $\chi^{\vee}$, see Lemma 2.2.7).

Definition 3.1.1 We say that the algebra $H$ is essential if, for each irreducible character $\chi \in \operatorname{Irr}(K(\mathbf{x}) H)$, the Schur element $s_{\chi}$ associated to $\chi$ is an element of $A$ of the form

$$
s_{\chi}=\xi_{\chi} N_{\chi} \prod_{i \in I_{\chi}} \Psi_{\chi, i}\left(M_{\chi, i}\right)^{n_{\chi, i}}
$$

where

(a) $\xi_{\chi}$ is an element of $R$,

(b) $N_{\chi}$ is a monomial in $A$,

(c) $I_{\chi}$ is an index set,

(d) $\left(\Psi_{\chi, i}\right)_{i \in I_{\chi}}$ is a family of monic polynomials in $R[x]$ of degree at least 1 , which are irreducible over $K$, prime to $x$ and $x-1$ and whose constant term is a unit in $R$,

(e) $\left(M_{\chi, i}\right)_{i \in I_{\chi}}$ is a family of primitive monomials in $A$, i.e., if $M_{\chi, i}=\prod_{i=0}^{m} x_{i}^{a_{i}}$, then $\operatorname{gcd}\left(a_{i}\right)=1$,

(f) $\left(n_{\chi, i}\right)_{i \in I_{\chi}}$ is a family of positive integers.

Following Theorem 1.5.6. Definition 3.1.1 describes the factorization of $s_{\chi}$ into irreducible factors in $K\left[\mathbf{x}, \mathbf{x}^{-1}\right]$. This factorization is unique. However, this does not mean that the monomials $M_{\chi, i}$ appearing in it are unique. Suppose that

$$
\Psi_{\chi}\left(M_{\chi}\right)=u \Phi_{\chi}\left(N_{\chi}\right)
$$

where

- $\Psi_{\chi}, \Phi_{\chi}$ are two $K$-irreducible polynomials as in Definition 3.1 .1 (d),

- $M_{\chi}, N_{\chi}$ are two primitive monomials in $A$, 
- $u$ is a unit of $K\left[\mathbf{x}, \mathbf{x}^{-1}\right]$.

Since the coefficients of $\Psi_{\chi}$ belong to $R$ and the constant term of $\Phi_{\chi}$ is a unit in $R$, we deduce that $u \in R\left[\mathbf{x}, \mathbf{x}^{-1}\right]$. Let $\varphi_{M}$ be an $R$-algebra morphism from $A$ to a Laurent polynomial ring $R\left[\mathbf{y}, \mathbf{y}^{-1}\right]$ in $m$ indeterminates associated with the monomial $M_{\chi}$ (recall Definition 1.4.3). If we apply $\varphi_{M}$ to the above equality, we obtain

$$
\Psi_{\chi}(1)=\varphi_{M}(u) \Phi_{\chi}\left(\varphi_{M}\left(N_{\chi}\right)\right) .
$$

The morphism $\varphi_{M}$ sends $u$ to a unit and $N_{\chi}$ to a monomial in $K\left[\mathbf{y}, \mathbf{y}^{-1}\right]$. Since $\Psi_{\chi}(1) \in R$ and $\Phi_{\chi}$ is prime to $x$, we deduce that $\varphi_{M}\left(N_{\chi}\right)=1$. By Proposition 1.4.6(2) and the fact that $N_{\chi}$ is primitive, we obtain that

$$
N_{\chi}=M_{\chi}^{ \pm 1}
$$

Now, if $M_{\chi}=N_{\chi}$, then $\Psi_{\chi}=\Phi_{\chi}$ and $u=1$. If $N_{\chi}=M_{\chi}^{-1}$, then $\operatorname{deg}\left(\Psi_{\chi}\right)=$ $\operatorname{deg}\left(\Phi_{\chi}\right)$ and $u=\Psi_{\chi}(0) M_{\chi}^{\operatorname{deg}\left(\Psi_{\chi}\right)}$. We summarize the above results into the following proposition.

Proposition 3.1.2 Let $\chi \in \operatorname{Irr}(K(\mathbf{x}) H)$ and assume that the Schur element $s_{\chi}$ associated to $\chi$ has the factorization described in Definition 3.1.1. Then

1. The monomials $\left(M_{\chi, i}\right)_{i \in I_{\chi}}$ are unique up to inversion.

2. The coefficient $\xi_{\chi}$ is unique up to multiplication by a unit of $R$.

Now let $\mathfrak{p}$ be a prime ideal of $R$. If $\Psi_{\chi, i}\left(M_{\chi, i}\right)$ is a factor of $s_{\chi}$ and $\Psi_{\chi, i}(1) \in \mathfrak{p}$, then the monomial $M_{\chi, i}$ is called $\mathfrak{p}$-essential for $\chi$ in $A$. By Proposition 1.4.6(1), we have

$$
\Psi_{\chi, i}(1) \in \mathfrak{p} \Leftrightarrow \Psi_{\chi, i}\left(M_{\chi, i}\right) \in \mathfrak{q}_{\chi, i}
$$

where $\mathfrak{q}_{\chi, i}:=\left(M_{\chi, i}-1\right) A+\mathfrak{p} A$. We deduce that $M_{\chi, i}$ is $\mathfrak{p}$-essential for $\chi$ if and only if $M_{\chi, i}^{-1}$ is $\mathfrak{p}$-essential for $\chi$.

Recall that, by Proposition 1.4.5, $\mathfrak{q}_{\chi, i}$ is a prime ideal of $A$. Due to the primeness of $\mathfrak{q}_{\chi, i}$ and the proposition above, the following result is an immediate consequence of $(\dagger)$.

Proposition 3.1.3 Let $M$ be a primitive monomial in $A$ and $\mathfrak{q}_{M}:=(M-$ 1) $A+\mathfrak{p} A$. Then $M$ is $\mathfrak{p}$-essential for $\chi$ in $A$ if and only if $s_{\chi} / \xi_{\chi} \in \mathfrak{q}_{M}$, where $\xi_{\chi}$ denotes the coefficient of $s_{\chi}$. 
Proof: If $M$ is a $\mathfrak{p}$-essential monomial for $\chi$ in $A$, then there exists a polynomial $\Psi$ as in Definition 3.1.1(d) such that $\Psi(M)$ is a factor of $s_{\chi}$ and $\Psi(1) \in \mathfrak{p}$. By $(\dagger)$, we have that $\Psi(M) \in \mathfrak{q}_{M}$, whence $s_{\chi} / \xi_{\chi} \in \mathfrak{q}_{M}$. Now, if $s_{\chi} / \xi_{\chi} \in \mathfrak{q}_{M}$, then, since $\mathfrak{q}_{M}$ is a prime ideal of $A$, there exists a polynomial $\Phi$ as in Definition 3.1.1(d) and a primitive monomial $N$ such that $\Phi(N)$ is an irreducible factor of $s_{\chi}$ and $\Phi(N) \in \mathfrak{q}_{M}$. Let $\varphi_{M}$ be an $R$-algebra morphism from $A$ to a Laurent polynomial ring $B$ in $m$ indeterminates associated with the monomial $M$. By Proposition 1.4.6(1), we obtain that $\varphi_{M}(\Phi(N))=\Phi\left(\varphi_{M}(N)\right) \in \mathfrak{p} B$. Since $\phi_{M}(N)$ is a monomial in $B$ and the constant term of $\Phi$ is a unit in $R$, we must have $\varphi_{M}(N)=1$ and $\Phi(1) \in \mathfrak{p}$. Since $N$ is primitive, Proposition 1.4.6 (2) implies that $N=M^{ \pm 1}$. By definition, $M$ is a $\mathfrak{p}$-essential monomial for $\chi$.

A primitive monomial $M$ in $A$ is called $\mathfrak{p}$-essential for $H$, if there exists an irreducible character $\chi \in \operatorname{Irr}(K(\mathbf{x}) H)$ such that $M$ is $\mathfrak{p}$-essential for $\chi$.

\subsection{Specialization via morphisms associated with monomials}

From now on, we assume that the algebra $H$ is essential. Let $M:=\prod_{i=0}^{m} x_{i}^{a_{i}}$ be a monomial in $A$ such that $a_{i} \in \mathbb{Z}$ and $\operatorname{gcd}\left(a_{i}\right)=1$, i.e., $M$ is primitive. Let $\mathbf{y}=\left(y_{j}\right)_{1 \leq j \leq m}$ be a set of $m$ indeterminates over $R$. We set $B:=R\left[y_{1}^{ \pm 1}, \ldots, y_{m}^{ \pm 1}\right]$ and consider $\varphi_{M}: A \rightarrow B$ an $R$-algebra morphism associated with $M$. Let us denote by $H_{\varphi_{M}}$ the algebra obtained from $H$ via the specialization $\varphi_{M}$. The algebra $H_{\varphi_{M}}$ has also a symmetrizing form defined as the specialization of $t$ via $\varphi_{M}$.

Proposition 3.2.1 The algebra $K(\mathbf{y}) H_{\varphi_{M}}$ is split semisimple.

Proof: By assumption, the algebra $K(\mathbf{x}) H$ is split semisimple. The ring $A$ is a Krull ring and $\operatorname{Ker} \varphi_{M}=(M-1) A$ is a prime ideal of height 1 of $A$. Let $\chi \in \operatorname{Irr}(K(\mathbf{x}) H)$. Using the same description for the Schur element $s_{\chi}$ associated to $\chi$ as in Definition 3.1.1, we obtain that

$$
\varphi_{M}\left(s_{\chi}\right)=\xi_{\chi} \varphi_{M}\left(N_{\chi}\right) \prod_{i \in I_{\chi}} \Psi_{\chi, i}\left(\varphi_{M}\left(M_{\chi, i}\right)\right)^{n_{\chi, i}} .
$$

Since $\varphi_{M}\left(N_{\chi}\right)$ and $\varphi_{M}\left(M_{\chi, i}\right)$ are monomials in $B$ and $\Psi_{\chi, i}(1) \neq 0$ for all $i \in I_{\chi}$, we deduce that $\varphi_{M}\left(s_{\chi}\right) \neq 0$. Thus, we can apply Theorem 2.4 .12 and obtain that the algebra $K(\mathbf{y}) H_{\varphi_{M}}$ is split semisimple. 
By "Tits' deformation theorem" (Theorem 2.4.9), the map $\varphi_{M}$ induces a bijection between the set of irreducible characters of the algebra $K(\mathbf{x}) H$ and the set of irreducible characters of the algebra $K(\mathbf{y}) H_{\varphi_{M}}$. The Schur elements of the latter are the specializations of the Schur elements of $K(\mathbf{x}) H$ via $\varphi_{M}$ and hence, $H_{\varphi_{M}}$ is also essential.

From now on, whenever we refer to irreducible characters, we mean irreducible characters of the algebra $K(\mathbf{x}) H$. Due to the bijection induced by "Tits' deformation theorem", we can compare the blocks of $H$ and $H_{\varphi_{M}}$ (in terms of partitions of $\operatorname{Irr}(K(\mathbf{x}) H))$ over suitable rings.

Let $\mathfrak{p}$ be a prime ideal of $R$ and $\mathfrak{q}_{M}:=(M-1) A+\mathfrak{p} A$.

Proposition 3.2.2 The blocks of $B_{\mathfrak{p} B} H_{\varphi_{M}}$ coincide with the blocks of $A_{\mathfrak{q}_{M}} H$.

Proof: Let us denote by $\mathfrak{n}_{M}$ the kernel of $\varphi_{M}$, i.e., $\mathfrak{n}_{M}:=(M-1) A$. By Proposition 1.4.6(1), we have that $A_{\mathfrak{q}_{M}} / \mathfrak{n}_{M} A_{\mathfrak{q}_{M}} \cong B_{\mathfrak{p} B}$. Proposition 2.1.13 implies that the canonical surjection $A_{\mathfrak{q}_{M}} H \rightarrow\left(A_{\mathfrak{q}_{M}} / \mathfrak{n}_{M} A_{\mathfrak{q}_{M}}\right) H$ induces a block bijection between these two algebras, whence the desired result.

Remark: Proposition 3.2 .2 implies that the $\mathfrak{p}$-blocks of an algebra obtained as the specialization of $H$ via a morphism associated with a monomial $M$ do not depend on the actual choice of the morphism.

Proposition 3.2.3 If two irreducible characters $\chi$ and $\psi$ are in the same block of $A_{\mathfrak{p} A} H$, then they are in the same block of $A_{\mathfrak{q}_{M}} H$.

Proof: Let $C$ be a block of $A_{\mathfrak{q}_{M}} H$. Then $\sum_{\chi \in C} e_{\chi} \in A_{\mathfrak{q}_{M}} H \subset A_{\mathfrak{p} A} H$. Thus $C$ is a union of blocks of $A_{\mathfrak{p} A} H$.

Corollary 3.2.4 If two irreducible characters $\chi$ and $\psi$ are in the same block of $A_{\mathfrak{p} A} H$, then they are in the same block of $B_{\mathfrak{p} B} H_{\varphi_{M}}$.

The corollary above implies that the size of $\mathfrak{p}$-blocks grows larger as the number of indeterminates becomes smaller. However, we will now see that the size of blocks remains the same, if our specialization is not associated with a p-essential monomial.

Proposition 3.2.5 Let $C$ be a block of $A_{\mathfrak{p} A} H$. If $M$ is not a $\mathfrak{p}$-essential monomial for any $\chi \in C$, then $C$ is a block of $A_{\mathfrak{q}_{M}} H$ (and thus of $B_{\mathfrak{p} B} H_{\varphi_{M}}$ ). 
Proof: $\quad$ Using the notations of Definition 3.1.1, Proposition 3.1.3 implies that, for all $\chi \in C$, we have $s_{\chi} / \xi_{\chi} \notin \mathfrak{q}_{M}$. Since $C$ is a block of $A_{\mathfrak{p} A} H$, we have

$$
\sum_{\chi \in C} e_{\chi}=\sum_{\chi \in C} \frac{\chi^{\vee}}{s_{\chi}} \in A_{\mathfrak{p} A} H .
$$

If $\mathcal{B}, \mathcal{B}^{\prime}$ are two $A$-bases of $H$ dual to each other, then $\chi^{\vee}=\sum_{b \in \mathcal{B}} \chi(b) b^{\prime}$ and the above relation implies that

$$
\sum_{\chi \in C} \frac{\chi(b)}{s_{\chi}} \in A_{\mathfrak{p} A}, \forall b \in \mathcal{B} .
$$

Set $f_{b}:=\sum_{\chi \in C}\left(\chi(b) / s_{\chi}\right) \in A_{\mathfrak{p} A}$. Then $f_{b}$ is of the form $r_{b} /(\xi s)$, where

$$
\xi:=\prod_{\chi \in C} \xi_{\chi} \in R \text { and } s:=\prod_{\chi \in C} s_{\chi} / \xi_{\chi} \in A .
$$

Since $\mathfrak{q}_{M}$ is a prime ideal of $A$, the element $s$, by assumption, doesn't belong to $\mathfrak{q}_{M}$. Moreover, we have that $r_{b} / \xi \in A_{\mathfrak{p} A}$. By Corollary 1.2.18, there exists $\xi^{\prime} \in R-\mathfrak{p}$ such that $r_{b} / \xi=r_{b}^{\prime} / \xi^{\prime}$ for some $r_{b}^{\prime} \in A$. Since $\mathfrak{q}_{M} \cap R=\mathfrak{p}$ (cf. Corollary 1.4.7), the element $\xi^{\prime}$ doesn't belong to the ideal $\mathfrak{q}_{M}$ either. Therefore, $f_{b}=r_{b}^{\prime} /\left(\xi^{\prime} s\right) \in A_{\mathfrak{q}_{M}} \forall b \in \mathcal{B}$, whence $\sum_{\chi \in C} e_{\chi} \in A_{\mathfrak{q}_{M}} H$. Thus, $C$ is a union of blocks of $A_{\mathfrak{q}_{M}} H$. Since the blocks of $A_{\mathfrak{q}_{M}} H$ are unions of blocks of $A_{\mathfrak{p} A} H$, by Proposition 3.2.3, we eventually obtain that $C$ is a block of $A_{\mathfrak{q}_{M}} H$.

Corollary 3.2.6 If $M$ is not a $\mathfrak{p}$-essential monomial for any $\chi \in \operatorname{Irr}(K(\mathbf{x}) H)$, then the blocks of $A_{\mathfrak{q}_{M}} H$ coincide with the blocks of $A_{\mathfrak{p} A} H$.

Of course, all the above results hold for $B$ in the place of $A$, if we further specialize $B$ (and $H_{\varphi_{M}}$ ) via a morphism associated with a monomial in $B$.

\subsection{Specialization via adapted morphisms}

For $r \in\{1, \ldots, m+1\}$, we set $C_{r}:=R\left[\mathbf{y}, \mathbf{y}^{-1}\right]$, where $\mathbf{y}:=\left(y_{j}\right)_{r \leq j \leq m}$ is a set of $m+1-r$ indeterminates over $R$. For $r=m+1, C_{r}=R$.

From now on, we fix $r \in\{1, \ldots, m+1\}$ and set $\mathcal{R}:=C_{r}$. We recall that an $R$-algebra morphism $\varphi: A \rightarrow \mathcal{R}$ is called adapted, if $\varphi=\varphi_{r} \circ \varphi_{r-1} \circ \ldots \circ \varphi_{1}$, where $\varphi_{i}$ is a morphism associated with a monomial for all $i=1, \ldots, r$. The family $\mathcal{F}:=\left\{\varphi_{r}, \varphi_{r-1}, \ldots, \varphi_{1}\right\}$ is called an adapted family for $\varphi$ whose initial morphism is $\varphi_{1}$. 
Let $\varphi: A \rightarrow \mathcal{R}$ be an adapted morphism and let us denote by $H_{\varphi}$ the algebra obtained as the specialization of $H$ via $\varphi$. Applying Proposition $3.2 .1 r$ times, we obtain that the algebra $K(\mathbf{y}) H_{\varphi}$ is split semisimple. By "Tits' deformation theorem", the morphism $\varphi$ induces a bijection from the set $\operatorname{Irr}(K(\mathbf{x}) H)$ to the set $\operatorname{Irr}\left(K(\mathbf{y}) H_{\varphi}\right)$ of irreducible characters of $K(\mathbf{y}) H_{\varphi}$. Therefore, whenever we refer to irreducible characters, we mean irreducible characters of the algebra $K(\mathbf{x}) H$.

If $M:=\prod_{i=0}^{m} x_{i}^{b_{i}}$ is a monomial such that $\operatorname{gcd}\left(b_{i}\right)=d \in \mathbb{Z}$, then we denote $M^{\circ}:=\prod_{i=0}^{m} x_{i}^{b_{i} / d}$. Once more, let $\mathfrak{p}$ be a prime ideal of $R$.

Proposition 3.3.1 Let $\varphi: A \rightarrow \mathcal{R}$ be an adapted morphism and $H_{\varphi}$ the algebra obtained as the specialization of $H$ via $\varphi$. If $M$ is a monomial in $A$ such that $\varphi(M)=1$ and $\mathfrak{q}_{M^{\circ}}:=\left(M^{\circ}-1\right) A+\mathfrak{p} A$, then the blocks of $\mathcal{R}_{\mathfrak{p R}} H_{\varphi}$ are unions of blocks of $A_{\mathfrak{q}_{M^{\circ}}} H$.

Proof: $\quad$ Let $M$ be a monomial in $A$ such that $\varphi(M)=1$. Due to proposition 1.4.11, there exists an adapted family for $\varphi$ whose initial morphism $\varphi_{1}$ is associated with $M^{\circ}$. Let us denote by $B$ the image of $\varphi_{1}$ and by $H_{\varphi_{1}}$ the algebra obtained as the specialization of $H$ via $\varphi_{1}$. Due to Proposition 3.2.2, the blocks of $B_{\mathfrak{p} B} H_{\varphi_{1}}$ coincide with the blocks of $A_{\mathfrak{q}_{M^{\circ}}} H$. Now, by corollary 3.2.4, if two irreducible characters belong to the same $\mathfrak{p}$-block of an essential algebra, then they belong to the same $\mathfrak{p}$-block of its specialization via a morphism associated with a monomial. Inductively, we obtain that the blocks of $\mathcal{R}_{\mathfrak{p} \mathcal{R}} H_{\varphi}$ are unions of blocks of $B_{\mathfrak{p} B} H_{\varphi_{1}}$ and thus of $A_{\mathfrak{q}_{M^{\circ}}} H$.

We will now state and prove our main result concerning the $\mathfrak{p}$-blocks of essential algebras. Let $\varphi: A \rightarrow \mathcal{R}$ be an adapted morphism and $H_{\varphi}$ the algebra obtained as the specialization of $H$ via $\varphi$. Let $M_{1}, \ldots, M_{k}$ be all the $\mathfrak{p}$-essential monomials for $H$ such that $\phi\left(M_{j}\right)=1$ for all $j=1, \ldots, k$. Note that we can easily find all $\mathfrak{p}$-essential monomials for $H$ by looking at the unique factorization of its Schur elements in $K\left[\mathbf{x}, \mathbf{x}^{-1}\right]$. We have $M_{j}^{\circ}=M_{j}$ for all $j=1, \ldots, k$. Set $\mathfrak{q}_{0}:=\mathfrak{p} A, \mathfrak{q}_{j}:=\mathfrak{p} A+\left(M_{j}-1\right) A$ for $j=1, \ldots, k$ and $\mathcal{Q}:=\left\{\mathfrak{q}_{0}, \mathfrak{q}_{1}, \ldots, \mathfrak{q}_{k}\right\}$.

Now let $\mathfrak{q} \in \mathcal{Q}$. If two irreducible characters $\chi, \psi$ belong to the same block of $A_{\mathfrak{q}} H$, we write $\chi \sim_{\mathfrak{q}} \psi$.

Theorem 3.3.2 Two irreducible characters $\chi, \psi \in \operatorname{Irr}(K(\mathbf{x}) H)$ are in the same block of $\mathcal{R}_{\mathfrak{p R}} H_{\varphi}$ if and only if there exist a finite sequence $\chi_{0}, \chi_{1}, \ldots, \chi_{n} \in$ $\operatorname{Irr}(K(\mathbf{x}) H)$ and a finite sequence $\mathfrak{q}_{j_{1}}, \ldots, \mathfrak{q}_{j_{n}} \in \mathcal{Q}$ such that

- $\chi_{0}=\chi$ and $\chi_{n}=\psi$, 
- for all $i(1 \leq i \leq n), \chi_{i-1} \sim_{\mathfrak{q}_{j_{i}}} \chi_{i}$.

Proof: Let us denote by $\sim$ the equivalence relation on $\operatorname{Irr}(K(\mathbf{x}) H)$ defined as the transitive closure of the relation "there exists $\mathfrak{q} \in \mathcal{Q}$ such that $\chi \sim_{\mathfrak{q}} \psi$ ". We have to show that $\chi$ and $\psi$ are in the same block of $\mathcal{R}_{\mathfrak{p R}} H_{\varphi}$ if and only if $\chi \sim \psi$.

If $\chi \sim \psi$, then Proposition 3.3 .1 implies that $\chi$ and $\psi$ are in the same block of $\mathcal{R}_{\mathfrak{p} \mathcal{R}} H_{\varphi}$. Now let $C$ be an equivalence class of $\sim$. We have that $C$ is a union of blocks of $A_{\mathfrak{q}} H$, for all $\mathfrak{q} \in \mathcal{Q}$. Therefore,

$$
\sum_{\theta \in C} \frac{\theta^{\vee}}{s_{\theta}} \in A_{\mathfrak{q}} H, \forall \mathfrak{q} \in \mathcal{Q} .
$$

If $\mathcal{B}, \mathcal{B}^{\prime}$ are two dual bases of $H$ with respect to the symmetrizing form $t$, then $\theta^{\vee}=\sum_{b \in \mathcal{B}} \theta(b) b^{\prime}$ and hence,

$$
\sum_{\theta \in C} \frac{\theta(b)}{s_{\theta}} \in A_{\mathfrak{q}}, \forall \mathfrak{q} \in \mathcal{Q}, \forall b \in \mathcal{B}
$$

Using the notations for $s_{\theta}$ of Definition 3.1.1, set $\xi_{C}:=\prod_{\theta \in C} \xi_{\theta}$ and $s_{C}:=$ $\prod_{\theta \in C}\left(s_{\theta} / \xi_{\theta}\right)$. Then, for all $b \in \mathcal{B}$, there exists an element $r_{C, b} \in A$ such that

$$
\sum_{\theta \in C} \frac{\theta(b)}{s_{\theta}}=\frac{r_{C, b}}{\xi_{C} s_{C}} .
$$

The element $s_{C} \in A$ is product of terms (monic $K$-irreducible polynomials taking values on monomials) which are irreducible in $K\left[\mathbf{x}, \mathbf{x}^{-1}\right]$, due to Theorem 1.5.6. We also have $s_{C} \notin \mathfrak{p} A$.

Fix $b \in \mathcal{B}$. The ring $K\left[\mathbf{x}, \mathbf{x}^{-1}\right]$ is a unique factorization domain and thus the quotient $r_{C, b} / s_{C}$ can be written uniquely in the form $r / \alpha s$, where

- $r, s \in A$,

- $\alpha \in R$,

- $s$ divides $s_{C}$ in $A$,

- $\operatorname{gcd}(r, s)=1$ in $K\left[\mathbf{x}, \mathbf{x}^{-1}\right]$.

Setting $\xi:=\alpha \xi_{C}$, we obtain

$$
\frac{r_{C, b}}{\xi_{C} s_{C}}=\frac{r}{\xi s} \in A_{\mathfrak{q}}, \forall \mathfrak{q} \in \mathcal{Q} .
$$


Thus, for all $\mathfrak{q} \in \mathcal{Q}$, there exist $r_{\mathfrak{q}}, s_{\mathfrak{q}} \in A$ with $s_{\mathfrak{q}} \notin \mathfrak{q}$ such that

$$
\frac{r}{\xi s}=\frac{r_{\mathfrak{q}}}{s_{\mathfrak{q}}}
$$

Since $\operatorname{gcd}(r, s)=1$, we obtain that $s$ divides $s_{\mathfrak{q}}$ in $K\left[\mathbf{x}, \mathbf{x}^{-1}\right]$. However, $s$ divides $s_{C}$ in $A$ and hence, $s$ is a product of monic $K$-irreducible polynomials taking values on monomials. Consequently, at least one of the coefficients of $s$ is a unit in $A$. Corollary 1.2.20 implies that $s$ divides $s_{\mathfrak{q}}$ in $A$. Therefore, $s \notin \mathfrak{q}$ for all $\mathfrak{q} \in \mathcal{Q}$.

Moreover, we have that $r / \xi \in A_{\mathfrak{q}_{0}}=A_{\mathfrak{p} A}$. By Corollary 1.2.18, there exist $r^{\prime} \in A$ and $\xi^{\prime} \in R-\mathfrak{p}$ such that $r / \xi=r^{\prime} / \xi^{\prime}$. Since $\mathfrak{q} \cap R=\mathfrak{p}$, we deduce that $\xi^{\prime} \notin \mathfrak{q}$ for all $\mathfrak{q} \in \mathcal{Q}$. Thus,

$$
\frac{r}{\xi s}=\frac{r^{\prime}}{\xi^{\prime} s} \in A_{\mathfrak{q}}, \forall \mathfrak{q} \in \mathcal{Q} .
$$

Now let us suppose that $\varphi\left(\xi^{\prime} s\right)=\xi^{\prime} \varphi(s)$ belongs to $\mathfrak{p} \mathcal{R}$. Since $\xi^{\prime} \notin \mathfrak{p}$, we must have $\varphi(s) \in \mathfrak{p} \mathcal{R}$. However, the morphism $\varphi$ sends every monomial of $A$ to a monomial in $\mathcal{R}$. Since $s \notin \mathfrak{p} A$ and $s$ divides $s_{C}, s$ must have a factor of the form $\Psi(M)$, where

- $M$ is a primitive monomial in $A$ such that $\varphi(M)=1$,

- $\Psi$ is a monic $K$-irreducible polynomial as in Definition 3.1.1(d) such that $\Psi(1) \in \mathfrak{p}$.

By Proposition 1.4.6(1), we obtain that $s \in \mathfrak{q}_{M}:=(M-1) A+\mathfrak{p} A$.

Since $s$ divides $s_{C}, \Psi(M)$ also divides $s_{C}$. By definition, $M$ is a $\mathfrak{p}$ essential monomial for some irreducible character $\theta \in C$. Consequently, $M \in\left\{M_{1}, \ldots, M_{k}\right\}$. This contradicts the fact that $s \notin \mathfrak{q}$ for all $\mathfrak{q} \in \mathcal{Q}$. Therefore, $\varphi\left(\xi^{\prime} s\right) \notin \mathfrak{p} \mathcal{R}$ and so we have

$$
\frac{\varphi\left(r_{C, b}\right)}{\varphi\left(\xi_{C} s_{C}\right)}=\frac{\varphi\left(r^{\prime}\right)}{\varphi\left(\xi^{\prime} s\right)} \in \mathcal{R}_{\mathfrak{p R}}
$$

This equality holds for all $b \in \mathcal{B}$ and hence,

$$
\sum_{\theta \in C} \frac{\varphi\left(\theta^{\vee}\right)}{\varphi\left(s_{\theta}\right)} \in \mathcal{R}_{\mathfrak{p} \mathcal{R}} H_{\varphi} .
$$

Thus, $C$ is a union of blocks of $\mathcal{R}_{\mathfrak{p} \mathcal{R}} H_{\varphi}$. 
Remark: We can obtain Corollary 3.2.6 as an application of the above theorem for $\mathcal{Q}=\left\{\mathfrak{q}_{0}\right\}$.

Theorem 3.3.2 allows us to calculate the blocks of $\mathcal{R}_{\mathfrak{p} \mathcal{R}} H_{\varphi}$ for any adapted morphism $\varphi: A \rightarrow \mathcal{R}$, if we know the blocks of $A_{\mathfrak{p} A} H$ and the blocks of $A_{\mathfrak{q}_{M}} H$ for all $\mathfrak{p}$-essential monomials $M$. Thus, the study of the blocks of the algebra $H$ in a finite number of cases gives us the $\mathfrak{p}$-blocks of all essential algebras obtained via such specializations.

\subsection{The map $I^{n}$}

Let $n$ be an integer, $n \neq 0$, and let $\mathbf{y}:=\left(y_{i}\right)_{0 \leq i \leq m}$ be a set of $m+1$ indeterminates over $R$. Define $I^{n}: A \rightarrow A^{\prime}:=R\left[\mathbf{y}, \mathbf{y}^{-1}\right]$ to be the $R$-algebra morphism $x_{i} \mapsto y_{i}^{n}$. Obviously, $I^{n}$ is injective. Therefore, if we denote by $H^{\prime}$ the algebra obtained as the specialization of $H$ via $I^{n}$, Corollary 2.4.11 implies that the algebra $K(\mathbf{y}) H^{\prime}$ is split semisimple. Again, by "Tits' deformation theorem", the morphism $I^{n}$ induces a bijection from the set $\operatorname{Irr}(K(\mathbf{x}) H)$ to the set $\operatorname{Irr}\left(K(\mathbf{y}) H^{\prime}\right)$. Again, let $\mathfrak{p}$ be a prime ideal of $R$.

Lemma 3.4.1 The blocks of $A_{\mathfrak{p} A^{\prime}}^{\prime} H^{\prime}$ coincide with the blocks of $A_{\mathfrak{p} A} H$.

Proof: $\quad$ Since the map $I^{n}$ is injective, we can consider $A$ as a subring of $A^{\prime}$ via the identification $x_{i}=y_{i}^{n}$ for all $i=0,1, \ldots, m$. By Corollary 1.1.3, we obtain that $A_{\mathfrak{p} A}$ is contained in $A_{\mathfrak{p} A^{\prime}}^{\prime}$ and hence, the blocks of $A_{\mathfrak{p} A} H$ are unions of blocks of $A_{\mathfrak{p} A^{\prime}}^{\prime} H^{\prime}$.

Now let $C$ be a block of $A_{\mathfrak{p} A^{\prime}}^{\prime} H^{\prime}$. Since the field of fractions of $A$ is a splitting field for $H$ (and thus for $H^{\prime}$ ), we obtain that

$$
\sum_{\chi \in C} e_{\chi} \in\left(A_{\mathfrak{p} A^{\prime}}^{\prime} \cap K(\mathbf{x})\right) H^{\prime}
$$

If $A_{\mathfrak{p} A^{\prime}}^{\prime} \cap K(\mathbf{x})=A_{\mathfrak{p} A}$, then $C$ is also a union of blocks of $A_{\mathfrak{p} A} H$ and we obtain the desired result.

In order to prove that $A_{\mathfrak{p} A^{\prime}}^{\prime} \cap K(\mathbf{x})=A_{\mathfrak{p} A}$, it suffices to show that:

(a) The ring $A_{\mathfrak{p} A}$ is integrally closed.

(b) The ring $A_{\mathfrak{p} A^{\prime}}^{\prime}$ is integral over $A_{\mathfrak{p} A}$.

Since the ring $A$ is integrally closed, (a) is immediate by Corollary 1.2.8, For (b), we have that $A^{\prime}$ is integral over $A$, since $y_{i}^{n}-x_{i}=0$, for all $i=0,1, \ldots, m$. Moreover, $A^{\prime}$ is integrally closed and thus the integral closure 
of $A$ in $K(\mathbf{y})$. The only prime ideal of $A^{\prime}$ lying over $\mathfrak{p} A$ is $\mathfrak{p} A^{\prime}$. Following Corollary 1.2.12, we obtain that the integral closure of $A_{\mathfrak{p} A}$ in $K(\mathbf{y})$ is $A_{\mathfrak{p} A^{\prime}}^{\prime}$. Thus, $A_{\mathfrak{p} A^{\prime}}^{\prime}$ is integral over $A_{\mathfrak{p} A}$.

We can consider $I^{n}$ as an endomorphism of $A$ and denote it by $I_{A}^{n}$. If $k$ is another non-zero integer, then $I_{A}^{k} \circ I_{A}^{n}=I_{A}^{n} \circ I_{A}^{k}=I_{A}^{k n}$. If now $\varphi: A \rightarrow \mathcal{R}$ is an adapted morphism, we can easily check that $\varphi \circ I_{A}^{n}=I_{\mathcal{R}}^{n} \circ \varphi$. Abusing notation, we write $\varphi \circ I^{n}=I^{n} \circ \varphi$.

Corollary 3.4.2 Let $\varphi: A \rightarrow \mathcal{R}$ be an adapted morphism and $H_{\varphi}$ the algebra obtained as the specialization of $H$ via $\varphi$. Let $\phi: A \rightarrow \mathcal{R}$ be an $R$-algebra morphism such that $I^{\alpha} \circ \varphi=I^{\beta} \circ \phi$ for some $\alpha, \beta \in \mathbb{Z} \backslash\{0\}$. If $H_{\phi}$ is the algebra obtained as the specialization of $H$ via $\phi$, then the blocks of $\mathcal{R}_{\mathfrak{p} \mathcal{R}} H_{\phi}$ coincide with the blocks of $\mathcal{R}_{\mathfrak{p} \mathcal{R}} H_{\varphi}$ and we can use Theorem 3.3.2 to calculate them. 


\section{Chapter 4}

\section{On Hecke algebras}

We will start this chapter by giving the definition and the classification of complex reflection groups. We will also define the braid group and the pure braid group associated to a complex reflection group. We will then introduce the generic Hecke algebra of a complex reflection group, which is a quotient of the group algebra of the associated braid group defined over a Laurent polynomial in a finite number of indeterminates. Under certain assumptions, which have been verified for all but a finite number of cases, we prove (Theorem 4.2.5) that the generic Hecke algebras of complex reflection groups are essential. Therefore, all results obtained in Chapter 3 apply to the case of the generic Hecke algebras.

A cyclotomic Hecke algebra is obtained from the generic Hecke algebra via a cyclotomic specialization (Definition 4.3.1). We prove (Theorem4.3.3) that any cyclotomic specialization is essentially an adapted morphism. Thus, we can use Theorem 3.3.2 in order to obtain the Rouquier blocks of a cyclotomic Hecke algebra (i.e., its blocks over the Rouquier ring, defined in section 4.4), which are a substitute for the families of characters that can be applied to all complex reflection groups. We will see that the Rouquier blocks have the property of semi-continuity, thus depending only on some "essential" hyperplanes for the group, which are determined by the generic Hecke algebra.

The theory developed in this chapter will allow us to determine the Rouquier blocks of the cyclotomic Hecke algebras of all (irreducible) complex reflection groups in the next and final chapter. 


\subsection{Complex reflection groups and associated braid groups}

Let $\mu_{\infty}$ be the group of all the roots of unity in $\mathbb{C}$ and $K$ a number field contained in $\mathbb{Q}\left(\mu_{\infty}\right)$. We denote by $\mu(K)$ the group of all the roots of unity of $K$. For every integer $d>1$, we set $\zeta_{d}:=\exp (2 \pi i / d)$ and denote by $\mu_{d}$ the group of all the $d$-th roots of unity. Let $V$ be a $K$-vector space of finite dimension $r$.

\subsubsection{Complex reflection groups}

Definition 4.1.1 A pseudo-reflection is a non-trivial element s of $\operatorname{GL}(V)$ which acts trivially on a hyperplane, called the reflecting hyperplane of $s$.

If $W$ is a finite subgroup of $\mathrm{GL}(V)$ generated by pseudo-reflections, then $(V, W)$ is called a $K$-reflection group of rank $r$.

We have the following classification of complex reflection groups, also known as the "Shephard-Todd classification". For more details about the classification, one may refer to [60].

Theorem 4.1.2 Let $(V, W)$ be an irreducible complex reflection group (i.e., $W$ acts irreducibly on $V$ ). Then one of the following assertions is true:

- There exist non-zero integers $d, e, r$ such that $(V, W) \cong G(d e, e, r)$, where $G(d e, e, r)$ is the group of all $r \times r$ monomial matrices with nonzero entries in $\mu_{d e}$ such that the product of all non-zero entries lies in $\mu_{d}$.

- $(V, W)$ is isomorphic to one of the 34 exceptional groups $G_{n}(n=$ $4, \ldots, 37)$.

Remark: Among the irreducible complex reflection groups, we encounter the irreducible real reflection groups. In particular, we have:

- $G(1,1, r) \cong A_{r-1}$ for $r \geq 2$,

- $G(2,1, r) \cong B_{r}\left(\right.$ or $\left.C_{r}\right)$ for $r \geq 2$,

- $G(2,2, r) \cong D_{r}$ for $r \geq 4$,

- $G(e, e, 2) \cong I_{2}(e)$, where $I_{2}(e)$ denotes the dihedral group of order $2 e$,

- $G_{23}=H_{3}, G_{28}=F_{4}, G_{30}=H_{4}, G_{35}=E_{6}, G_{36}=E_{7}, G_{37}=E_{8}$. 
The following theorem has been proved (using a case by case analysis) by Benard ([5]) and Bessis ([7]) and generalizes a well known result for Weyl groups.

Theorem-Definition 4.1.3 Let $(V, W)$ be a reflection group. Let $K$ be the field generated by the traces on $V$ of all the elements of $W$. Then all irreducible $K W$-representations are absolutely irreducible i.e., $K$ is a splitting field for $W$. The field $K$ is called the field of definition of the group $W$.

- If $K \subseteq \mathbb{R}$, then $W$ is a (finite) Coxeter group.

- If $K=\mathbb{Q}$, then $W$ is a Weyl group.

\subsubsection{Braid groups associated to complex reflection groups}

For all definitions and results about braid groups we follow [21]. Note that for a given topological space $X$ and a point $x_{0} \in X$, we denote by $\Pi_{1}\left(X, x_{0}\right)$ the fundamental group with base point $x_{0}$.

Let $V$ be a $K$-vector space of finite dimension $r$. Let $W$ be a finite subgroup of $\mathrm{GL}(V)$ generated by pseudo-reflections and acting irreducibly on $V$. We denote by $\mathcal{A}$ the set of its reflecting hyperplanes. We define the regular variety $V^{\text {reg }}:=\mathbb{C} \otimes V-\bigcup_{H \in \mathcal{A}} \mathbb{C} \otimes H$. For $x_{0} \in V^{\text {reg }}$, we define $P:=$ $\Pi_{1}\left(V^{\text {reg }}, x_{0}\right)$ the pure braid group (at $\left.x_{0}\right)$ associated with $W$. If $p: V^{\text {reg }} \rightarrow$ $V^{\mathrm{reg}} / W$ denotes the canonical surjection, we define $B:=\Pi_{1}\left(V^{\mathrm{reg}} / W, p\left(x_{0}\right)\right)$ the braid group (at $x_{0}$ ) associated with $W$.

The projection $p$ induces a surjective map $B \rightarrow W, \sigma \mapsto \bar{\sigma}$ as follows: Let $\tilde{\sigma}:[0,1] \rightarrow V^{\text {reg }}$ be a path in $V^{\text {reg }}$ such that $\tilde{\sigma}(0)=x_{0}$, which lifts $\sigma$. Then $\bar{\sigma}$ is defined by the equality $\bar{\sigma}\left(x_{0}\right)=\tilde{\sigma}(1)$. Note that the map $\sigma \mapsto \bar{\sigma}$ is an anti-morphism.

Denoting by $W^{\text {op }}$ the group opposite to $W$, we have the following short exact sequence

$$
1 \rightarrow P \rightarrow B \rightarrow W^{\mathrm{op}} \rightarrow 1,
$$

where the map $B \rightarrow W^{\text {op }}$ is defined by $\sigma \mapsto \bar{\sigma}$.

Now, for every hyperplane $H \in \mathcal{A}$, we set $e_{H}$ the order of the group $W_{H}$, where $W_{H}$ is the subgroup of $W$ formed by $\operatorname{id}_{V}$ and all the reflections fixing the hyperplane $H$. The group $W_{H}$ is cyclic: if $s_{H}$ denotes an element of $W_{H}$ with determinant $\zeta_{H}:=\zeta_{e_{H}}$, then $W_{H}=<s_{H}>$ and $s_{H}$ is called a distinguished reflection in $W$.

Let $L_{H}:=\operatorname{Im}\left(s-\mathrm{id}_{V}\right)$. Then, for all $x \in V$, we have $x=\operatorname{pr}_{H}(x)+\operatorname{pr}_{L_{H}}(x)$ with $\operatorname{pr}_{H}(x) \in H$ and $\operatorname{pr}_{L_{H}}(x) \in L_{H}$. Thus, $s_{H}(x)=\operatorname{pr}_{H}(x)+\zeta_{H} \operatorname{pr}_{L_{H}}(x)$. 
If $t \in \mathbb{R}$, we set $\zeta_{H}^{t}:=\exp \left(2 \pi i t / e_{H}\right)$ and we denote by $s_{H}^{t}$ the element of $\mathrm{GL}(V)$ (a pseudo-reflection if $t \neq 0$ ) defined by

$$
s_{H}^{t}(x):=\operatorname{pr}_{H}(x)+\zeta_{H}^{t} \operatorname{pr}_{L_{H}}(x) .
$$

For $x \in V$, we denote by $\sigma_{H, x}$ the path in $V$ from $x$ to $s_{H}(x)$ defined by

$$
\sigma_{H, x}:[0,1] \rightarrow V, t \mapsto s_{H}^{t}(x) .
$$

Let $\gamma$ be a path in $V^{\text {reg }}$ with initial point $x_{0}$ and terminal point $x_{H}$. Then $\gamma^{-1}$ is the path in $V^{\text {reg }}$ with initial point $x_{H}$ and terminal point $x_{0}$ such that

$$
\gamma^{-1}(t)=\gamma(1-t) \text { for all } t \in[0,1] .
$$

Thus, we can define the path $s_{H}\left(\gamma^{-1}\right): t \mapsto s_{H}\left(\gamma^{-1}(t)\right)$, which goes from $s_{H}\left(x_{H}\right)$ to $s_{H}\left(x_{0}\right)$ and lies also in $V^{\text {reg }}$, since for all $x \in V^{\text {reg }}, s_{H}(x) \in V^{\text {reg }}$ (If $s_{H}(x) \notin V^{\mathrm{reg}}$, then $s_{H}(x)$ must belong to a hyperplane $H^{\prime}$. If $s_{H^{\prime}}$ is a distinguished pseudo-reflection with reflecting hyperplane $H^{\prime}$, then $s_{H^{\prime}}\left(s_{H}(x)\right)=$ $s_{H}(x)$ and $s_{H}^{-1}\left(s_{H^{\prime}}\left(s_{H}(x)\right)\right)=x$. However, $s_{H}^{-1} s_{H^{\prime}} s_{H}$ is a reflection and $x$ belongs to its reflecting hyperplane, $s_{H}^{-1}\left(H^{\prime}\right)$. This contradicts the fact that $x$ belongs to $V^{\text {reg }}$.). Now we define a path from $x_{0}$ to $s_{H}\left(x_{0}\right)$ as follows:

$$
\sigma_{H, \gamma}:=s_{H}\left(\gamma^{-1}(t)\right) \cdot \sigma_{H, x_{H}} \cdot \gamma
$$

If $x_{H}$ is chosen "close to $H$ and far from the other reflecting hyperplanes", the path $\sigma_{H, \gamma}$ lies in $V^{\text {reg }}$ and its homotopy class does not depend on the choice of $x_{H}$. The element it induces in the braid group $B, \mathbf{s}_{H, \gamma}$, is a distinguished braid reflection around the image of $H$ in $V^{\text {reg }} / W$.

\section{Proposition 4.1.4}

1. The braid group $B$ is generated by the distinguished braid reflections around the images of the hyperplanes $H \in \mathcal{A}$ in $V^{\mathrm{reg}} / W$.

2. The image of $\mathbf{s}_{H, \gamma}$ in $W$ is $s_{H}$.

3. Whenever $\gamma^{\prime}$ is a path in $V^{\mathrm{reg}}$ from $x_{0}$ to $x_{H}$, if $\lambda$ denotes the loop in $V^{\text {reg }}$ defined by $\lambda:=\gamma^{\prime-1} \gamma$, then

$$
\sigma_{H, \gamma^{\prime}}=s_{H}(\lambda) \cdot \sigma_{H, \gamma} \cdot \lambda^{-1}
$$

In particular, $\mathbf{s}_{H, \gamma}$ and $\mathbf{s}_{H, \gamma}$ are conjugate in $P$.

4. The path $\prod_{j=e_{H}-1}^{j=0} \sigma_{H, s_{H}^{j}(\gamma)}$, a loop in $V^{\mathrm{reg}}$, induces the element $\mathbf{s}_{H, \gamma}^{e_{H}}$ in the braid group $B$ and belongs to the pure braid group $P$. It is a distinguished braid reflection around $H$ in $P$. 
Definition 4.1.5 Let $s$ be a distinguished pseudo-reflection in $W$ with reflecting hyperplane $H$. An s-distinguished braid reflection or monodromy generator is a distinguished braid reflection $\mathbf{s}$ around the image of $H$ in $V^{\mathrm{reg}} / W$ such that $\overline{\mathbf{s}}=s$.

Definition 4.1.6 Let $x_{0} \in V^{\text {reg }}$ as before. We denote by $\tau$ the element of $P$ defined by the loop $t \mapsto x_{0} \exp (2 \pi i t)$.

Lemma 4.1.7 We have $\tau \in Z P$.

Theorem-Definition 4.1.8 Given $\mathcal{C} \in \mathcal{A} / W$, there exists a unique length function $l_{\mathcal{C}}: B \rightarrow \mathbb{Z}$ defined as follows: if $b=\mathbf{s}_{1}^{n_{1}} \cdot \mathbf{s}_{2}^{n_{2}} \cdots \mathbf{s}_{m}^{n_{m}}$ where (for all j) $n_{j} \in \mathbb{Z}$ and $\mathbf{s}_{j}$ is a distinguished braid reflection around an element of $\mathcal{C}_{j}$, then

$$
l_{\mathcal{C}}(b)=\sum_{\left\{j \mid \mathcal{C}_{j}=\mathcal{C}\right\}} n_{j} .
$$

The length function $l: B \rightarrow \mathbb{Z}$ is defined, for all $b \in B$, as

$$
l(b)=\sum_{\mathcal{C} \in \mathcal{A} / W} l_{\mathcal{C}}(b) .
$$

We say that $B$ has an Artin-like presentation (cf. [56], 5.2), if it has a presentation of the form

$$
<\mathbf{s} \in \mathbf{S} \mid\left\{\mathbf{v}_{i}=\mathbf{w}_{i}\right\}_{i \in I}>,
$$

where $\mathbf{S}$ is a finite set of distinguished braid reflections and $I$ is a finite set of relations which are multi-homogeneous, i.e., such that, for each $i, \mathbf{v}_{i}$ and $\mathbf{w}_{i}$ are positive words in elements of $\mathbf{S}$ (and hence, for each $\mathcal{C} \in \mathcal{A} / W$, we have $\left.l_{\mathcal{C}}\left(\mathbf{v}_{i}\right)=l_{\mathcal{C}}\left(\mathbf{w}_{i}\right)\right)$.

The following result by Bessis ([8], Theorem 0.1) shows that any braid group has an Artin-like presentation.

Theorem 4.1.9 Let $W$ be a complex reflection group with associated braid group $B$. Then there exists a subset $\mathbf{S}=\left\{\mathbf{s}_{1}, \ldots, \mathbf{s}_{n}\right\}$ of $B$ such that

1. The elements $\mathbf{s}_{1}, \ldots, \mathbf{s}_{n}$ are distinguished braid reflection and therefore, their images $s_{1}, \ldots, s_{n}$ in $W$ are distinguished reflections.

2. The set $\mathbf{S}$ generates $B$ and therefore, $S:=\left\{s_{1}, \ldots, s_{n}\right\}$ generates $W$.

3. There exists a set $\mathcal{R}$ of relations of the form $\mathbf{w}_{1}=\mathbf{w}_{2}$, where $\mathbf{w}_{1}$ and $\mathbf{w}_{2}$ are positive words of equal length in the elements of $\mathbf{S}$, such that $<\mathbf{S} \mid \mathcal{R}>$ is a presentation of $B$. 
4. Viewing now $\mathcal{R}$ as a set of relations in $S$, the group $W$ is presented by

$$
<S \mid \mathcal{R} ;(\forall s \in S)\left(s^{e_{s}}=1\right)>
$$

where $e_{s}$ denotes the order of $s$ in $W$.

\subsection{Generic Hecke algebras}

Let $K, V, W, \mathcal{A}, P, B$ be defined as in the previous section. For every orbit $\mathcal{C}$ of $W$ on $\mathcal{A}$, we set $e_{\mathcal{C}}$ the common order of the subgroups $W_{H}$, where $H$ is any element of $\mathcal{C}$ and $W_{H}$ the subgroup formed by $\operatorname{id}_{V}$ and all the reflections fixing the hyperplane $H$.

We choose a set of indeterminates $\mathbf{u}=\left(u_{\mathcal{C}, j}\right)_{(\mathcal{C} \in \mathcal{A} / W)\left(0 \leq j \leq e_{\mathcal{C}}-1\right)}$ and we denote by $\mathbb{Z}\left[\mathbf{u}, \mathbf{u}^{-1}\right]$ the Laurent polynomial ring in all the indeterminates $\mathbf{u}$. We define the generic Hecke algebra $\mathcal{H}$ of $W$ to be the quotient of the group algebra $\mathbb{Z}\left[\mathbf{u}, \mathbf{u}^{-1}\right] B$ by the ideal generated by the elements of the form

$$
\left(\mathbf{s}-u_{\mathcal{C}, 0}\right)\left(\mathbf{s}-u_{\mathcal{C}, 1}\right) \ldots\left(\mathbf{s}-u_{\mathcal{C}, e_{\mathcal{C}}-1}\right),
$$

where $\mathcal{C}$ runs over the set $\mathcal{A} / W$ and s runs over the set of monodromy generators around the images in $V^{\text {reg }} / W$ of the elements of the hyperplane orbit $\mathcal{C}$.

Example 4.2.1 Let $W:=G_{2}=<s, t \mid$ ststst $=t s t s t s, s^{2}=t^{2}=1>$ be the dihedral group of order 12. Then the generic Hecke algebra of $W$ is defined over the Laurent polynomial ring in four indeterminates $\mathbb{Z}\left[u_{0}, u_{0}^{-1}, u_{1}, u_{1}^{-1}, w_{0}, w_{0}^{-1}, w_{1}, w_{1}^{-1}\right]$ and can be presented as follows:

$$
\begin{aligned}
& \mathcal{H}\left(G_{2}\right)=<S, T \mid \quad S T S T S T=T S T S T S, \quad\left(S-u_{0}\right)\left(S-u_{1}\right)=0, \\
& \left(T-w_{0}\right)\left(T-w_{1}\right)=0>\text {. }
\end{aligned}
$$

Example 4.2.2 Let $W:=G_{4}=<s, t \mid s t s=t s t, s^{3}=t^{3}=1>$. Then $s$ and $t$ are conjugate in $W$ and their reflecting hyperplanes belong to the same orbit of $W$ on $\mathcal{A}$. The generic Hecke algebra of $W$ can be presented as follows:

$$
\begin{array}{ll}
\mathcal{H}\left(G_{4}\right)=<S, T \mid S T S=T S T, & \left(S-u_{0}\right)\left(S-u_{1}\right)\left(S-u_{2}\right)=0, \\
& \left(T-u_{0}\right)\left(T-u_{1}\right)\left(T-u_{2}\right)=0>.
\end{array}
$$

We make some assumptions for the generic Hecke algebra $\mathcal{H}$. Note that they have been verified for all but a finite number of irreducible complex reflection groups ([20], remarks before 1.17, §2; [35]).

Assumptions 4.2.3 The algebra $\mathcal{H}$ is a free $\mathbb{Z}\left[\mathbf{u}, \mathbf{u}^{-1}\right]$-module of rank $|W|$. Moreover, there exists a linear form $t: \mathcal{H} \rightarrow \mathbb{Z}\left[\mathbf{u}, \mathbf{u}^{-1}\right]$ with the following properties: 
1. $t$ is a symmetrizing form on $\mathcal{H}$, i.e., $t\left(h h^{\prime}\right)=t\left(h^{\prime} h\right)$ for all $h, h^{\prime} \in \mathcal{H}$ and the map

$$
\begin{aligned}
\hat{t}: \mathcal{H} & \rightarrow \operatorname{Hom}\left(\mathcal{H}, \mathbb{Z}\left[\mathbf{u}, \mathbf{u}^{-1}\right]\right) \\
h & \mapsto \quad\left(h^{\prime} \mapsto t\left(h h^{\prime}\right)\right)
\end{aligned}
$$

is an isomorphism.

2. Via the specialization $u_{\mathcal{C}, j} \mapsto \zeta_{e_{\mathcal{C}}}^{j}$, the form $t$ becomes the canonical symmetrizing form on the group algebra $\mathbb{Z}_{K}[W]$.

3. If we denote by $\alpha \mapsto \alpha^{*}$ the automorphism of $\mathbb{Z}\left[\mathbf{u}, \mathbf{u}^{-1}\right]$ consisting of the simultaneous inversion of the indeterminates, then for all $b \in B$, we have

$$
t\left(b^{-1}\right)^{*}=\frac{t(b \tau)}{t(\tau)}
$$

where $\tau$ is the (central) element of $P$ defined by the loop $t \mapsto x_{0} \exp (2 \pi i t)$.

We know that the form $t$ is unique $([20,2.1)$. From now on, we suppose that the assumptions 4.2 .3 are satisfied. Then we have the following result by G. Malle ([49], 5.2).

Theorem 4.2.4 Let $\mathbf{v}=\left(v_{\mathcal{C}, j}\right)_{(\mathcal{C} \in \mathcal{A} / W)\left(0 \leq j \leq e_{\mathcal{C}}-1\right)}$ be a set of $\sum_{\mathcal{C} \in \mathcal{A} / W} e_{\mathcal{C}}$ indeterminates such that, for every $\mathcal{C}, j$, we have $v_{\mathcal{C}, j}^{|\mu(K)|}=\zeta_{e_{\mathcal{C}}}^{-j} u_{\mathcal{C}, j}$. Then the $K(\mathbf{v})$-algebra $K(\mathbf{v}) \mathcal{H}$ is split semisimple.

By "Tits' deformation theorem" (Theorem 2.4.9), it follows that the specialization $v_{\mathcal{C}, j} \mapsto 1$ induces a bijection $\chi_{\mathbf{v}} \mapsto \chi$ from the $\operatorname{set} \operatorname{Irr}(K(\mathbf{v}) \mathcal{H})$ of absolutely irreducible characters of $K(\mathbf{v}) \mathcal{H}$ to the set $\operatorname{Irr}(W)$ of absolutely irreducible characters of $W$, such that the following diagram is commutative

$$
\begin{array}{cccc}
\chi_{\mathbf{v}}: & \mathcal{H} & \rightarrow & \mathbb{Z}_{K}\left[\mathbf{v}, \mathbf{v}^{-1}\right] \\
& \downarrow & & \downarrow \\
\chi: & \mathbb{Z}_{K}[W] & \rightarrow & \mathbb{Z}_{K} .
\end{array}
$$

Since the assumptions 4.2 .3 are satisfied and the algebra $K(\mathbf{v}) \mathcal{H}$ is split semisimple, we can define the Schur element $s_{\chi}(\mathbf{v})$ for every irreducible character $\chi_{\mathbf{v}}$ of $K(\mathbf{v}) \mathcal{H}$ with respect to the symmetrizing form $t$. The following result describes the form of the Schur elements associated to the irreducible characters of $K(\mathbf{v}) \mathcal{H}$.

Theorem 4.2.5 The Schur element $s_{\chi}(\mathbf{v})$ associated to the irreducible character $\chi_{\mathbf{v}}$ of $K(\mathbf{v}) \mathcal{H}$ is an element of $\mathbb{Z}_{K}\left[\mathbf{v}, \mathbf{v}^{-1}\right]$ of the form

$$
s_{\chi}(\mathbf{v})=\xi_{\chi} N_{\chi} \prod_{i \in I_{\chi}} \Psi_{\chi, i}\left(M_{\chi, i}\right)^{n_{\chi, i}}
$$

where 
(a) $\xi_{\chi}$ is an element of $\mathbb{Z}_{K}$,

(b) $N_{\chi}=\prod_{\mathcal{C}, j} v_{\mathcal{C}, j}^{b_{\mathcal{C}, j}}$ is a monomial in $\mathbb{Z}_{K}\left[\mathbf{v}, \mathbf{v}^{-1}\right]$ with $\sum_{j=0}^{e_{\mathcal{C}}-1} b_{\mathcal{C}, j}=0$ for all $\mathcal{C} \in \mathcal{A} / W$

(c) $I_{\chi}$ is an index set,

(d) $\left(\Psi_{\chi, i}\right)_{i \in I_{\chi}}$ is a family of $K$-cyclotomic polynomials in one variable (i.e., minimal polynomials of the roots of unity over $K$ ),

(e) $\left(M_{\chi, i}\right)_{i \in I_{\chi}}$ is a family of monomials in $\mathbb{Z}_{K}\left[\mathbf{v}, \mathbf{v}^{-1}\right]$ such that if $M_{\chi, i}=$ $\prod_{\mathcal{C}, j} v_{\mathcal{C}, j}^{a_{\mathcal{C}, j}}$, then $\operatorname{gcd}\left(a_{\mathcal{C}, j}\right)=1$ and $\sum_{j=0}^{e_{\mathcal{C}}-1} a_{\mathcal{C}, j}=0$ for all $\mathcal{C} \in \mathcal{A} / W$,

(f) $\left(n_{\chi, i}\right)_{i \in I_{\chi}}$ is a family of positive integers.

Proof: By Proposition 2.2.10, we have that $s_{\chi}(\mathbf{v}) \in \mathbb{Z}_{K}\left[\mathbf{v}, \mathbf{v}^{-1}\right]$. The rest is a case by case analysis: Let us first consider the group $G(d, 1, r)$. The Schur elements of $\mathcal{H}(G(d, 1, r))$ have been calculated independently by Geck, Iancu and Malle ([35]) and by Mathas ([53]). Following Theorem 6.7.2, they are obviously of the desired form. Moreover, in the Appendix we give the generic Schur elements for the groups $G(2 d, 2,2), G_{7}, G_{11}, G_{19}, G_{26}, G_{32}$ (calculated by Malle in [48] and [50]) and $F_{4}$ (calculated by Lusztig in [46]) and show that they are of the form described above. In the Appendix, we also give the specializations of the parameters which make

- $\mathcal{H}(G(d e, 1, r))$ the twisted symmetric algebra of the cyclic group $C_{e}$ over $\mathcal{H}(G(d e, e, r))$ in the case where $r>2$ or $r=2$ and $e$ is odd.

- $\mathcal{H}(G(d e, 2,2))$ the twisted symmetric algebra of the cyclic group $C_{e / 2}$ over $\mathcal{H}(G(d e, e, 2))$ in the case where $e$ is even.

- $\mathcal{H}\left(G_{7}\right)$ the twisted symmetric algebra of some finite cyclic group over $\mathcal{H}\left(G_{4}\right), \mathcal{H}\left(G_{5}\right)$ and $\mathcal{H}\left(G_{6}\right)$.

- $\mathcal{H}\left(G_{11}\right)$ the twisted symmetric algebra of some finite cyclic group over $\mathcal{H}\left(G_{8}\right), \mathcal{H}\left(G_{9}\right), \mathcal{H}\left(G_{10}\right), \mathcal{H}\left(G_{12}\right), \mathcal{H}\left(G_{13}\right), \mathcal{H}\left(G_{14}\right)$ and $\mathcal{H}\left(G_{15}\right)$.

- $\mathcal{H}\left(G_{19}\right)$ the twisted symmetric algebra of some finite cyclic group over $\mathcal{H}\left(G_{16}\right), \mathcal{H}\left(G_{17}\right), \mathcal{H}\left(G_{18}\right), \mathcal{H}\left(G_{20}\right), \mathcal{H}\left(G_{21}\right)$ and $\mathcal{H}\left(G_{22}\right)$.

- $\mathcal{H}\left(G_{26}\right)$ the twisted symmetric algebra of the cyclic group $C_{2}$ over $\mathcal{H}\left(G_{25}\right)$. 
In all these cases, Proposition 2.3.15 implies that the Schur elements of the twisted symmetric algebra are scalar multiples of the Schur elements of the subalgebra. Due to the nature of the specializations (each indeterminate is sent to an indeterminate or a root of unity or a product of the two), the Schur elements of the subalgebra are also of the desired form.

Finally, if $W$ is one of the remaining exceptional irreducible complex reflection groups, then $W$ has one hyperplane orbit $\mathcal{C}$ with $e_{\mathcal{C}}=2$. The generic Hecke algebra of $W$ is defined over a Laurent polynomial ring in two indeterminates $v_{\mathcal{C}, 0}$ and $v_{\mathcal{C}, 1}$. Its Schur elements should be products of $K$-cyclotomic polynomials in one variable $v:=v_{\mathcal{C}, 0} v_{\mathcal{C}, 1}^{-1}$. The generic Schur elements have been calculated

- for $E_{6}$ and $E_{7}$ by Surowski ([61]),

- for $E_{8}$ by Benson ([6]),

- for $H_{3}$ by Lusztig ([43]),

- for $H_{4}$ by Alvis and Lusztig ([1]),

- for $G_{24}, G_{27}, G_{29}, G_{31}, G_{33}$ and $G_{34}$ by Malle ([50]),

and they are, indeed, products of $K$-cyclotomic polynomials in "one" variable.

Note that in order to write the Schur elements in the desired form, we have used the GAP Package CHEVIE (where some mistakes in the articles cited above have been corrected).

Remark: It is a consequence of [58], Theorem 3.5, that the irreducible factors of the generic Schur elements over $\mathbb{C}\left[\mathbf{v}, \mathbf{v}^{-1}\right]$ are divisors of Laurent polynomials of the form $M(\mathbf{v})^{n}-1$, where

- $M(\mathbf{v})$ is a monomial in $\mathbb{C}\left[\mathbf{v}, \mathbf{v}^{-1}\right]$,

- $n$ is a positive integer.

We have seen that the specialization $v_{\mathcal{C}, j} \mapsto 1$ induces a bijection $\chi_{\mathbf{v}} \mapsto \chi$ from $\operatorname{Irr}(K(\mathbf{v}) \mathcal{H})$ to $\operatorname{Irr}(W)$. Due to the assumptions 4.2.3, it sends $s_{\chi}(\mathbf{v})$ to $|W| / \chi(1)$, which is the Schur element of $\chi$ with respect to the canonical symmetrizing form. Therefore, the first cyclotomic polynomial does not appear in the factorization of $s_{\chi}(\mathbf{v})$ (otherwise the specialization $v_{\mathcal{C}, j} \mapsto 1$ would send $s_{\chi}(\mathbf{v})$ to 0$)$.

The following result is an immediate application of Definition 3.1.1 
Theorem 4.2.6 The algebra $\mathcal{H}$, defined over the ring $\mathbb{Z}_{K}\left[\mathbf{v}, \mathbf{v}^{-1}\right]$, is an essential algebra.

Thanks to Theorem 4.2.6, all the results of Chapter 3 can be applied to the generic Hecke algebra of an irreducible complex reflection group.

Definition 4.2.7 Let $\mathfrak{p}$ be a prime ideal of $\mathbb{Z}_{K}$. We say that a (primitive) monomial $M$ in $\mathbb{Z}_{K}\left[\mathbf{v}, \mathbf{v}^{-1}\right]$ is $\mathfrak{p}$-essential for $W$, if $M$ is $\mathfrak{p}$-essential for $\mathcal{H}$.

Example 4.2.8 Let $W:=G_{2}$. The group $G_{2}$ is a Weyl group. We have seen that

$$
\begin{array}{ll}
\mathcal{H}\left(G_{2}\right)=<S, T \mid S T S T S T=T S T S T S, & \left(S-u_{0}\right)\left(S-u_{1}\right)=0, \\
& \left(T-w_{0}\right)\left(T-w_{1}\right)=0>.
\end{array}
$$

Set $x_{0}^{2}:=u_{0}, x_{1}^{2}:=-u_{1}, y_{0}^{2}:=w_{0}, y_{1}^{2}:=-w_{1}$. By Theorem 4.2.4, the algebra $\mathbb{Q}\left(x_{0}, x_{1}, y_{0}, y_{1}\right) \mathcal{H}\left(G_{2}\right)$ is split semisimple and hence, there exists a bijection between its irreducible characters and the irreducible characters of $G_{2}$. The group $G_{2}$ has 4 irreducible characters of degree 1 and 2 irreducible characters of degree 2. Set

$$
\begin{aligned}
& s_{1}\left(x_{0}, x_{1}, y_{0}, y_{1}\right):=\Phi_{4}\left(x_{0} x_{1}^{-1}\right) \cdot \Phi_{4}\left(y_{0} y_{1}^{-1}\right) \cdot \Phi_{3}\left(x_{0} x_{1}^{-1} y_{0} y_{1}^{-1}\right) \cdot \Phi_{6}\left(x_{0} x_{1}^{-1} y_{0} y_{1}^{-1}\right), \\
& s_{2}\left(x_{0}, x_{1}, y_{0}, y_{1}\right):=2 x_{0}^{-2} x_{1}^{2} \cdot \Phi_{3}\left(x_{0} x_{1}^{-1} y_{0} y_{1}^{-1}\right) \cdot \Phi_{6}\left(x_{0} x_{1}^{-1} y_{0}^{-1} y_{1}\right) \\
& \text { where } \Phi_{3}(x)=x^{2}+x+1, \Phi_{4}(x)=x^{2}+1, \Phi_{6}(x)=x^{2}-x+1 .
\end{aligned}
$$

The Schur elements of $\mathcal{H}\left(G_{2}\right)$ are

$$
\begin{gathered}
s_{1}\left(x_{0}, x_{1}, y_{0}, y_{1}\right), s_{1}\left(x_{0}, x_{1}, y_{1}, y_{0}\right), s_{1}\left(x_{1}, x_{0}, y_{0}, y_{1}\right), s_{1}\left(x_{1}, x_{0}, y_{1}, y_{0}\right), \\
s_{2}\left(x_{0}, x_{1}, y_{0}, y_{1}\right), s_{2}\left(x_{0}, x_{1}, y_{1}, y_{0}\right) .
\end{gathered}
$$

Since $\Phi_{3}(1)=3, \Phi_{4}(1)=2$ and $\Phi_{6}(1)=1$, we obtain that

- the (2)-essential monomials for $G_{2}$ are $x_{0} x_{1}^{-1}$ and $y_{0} y_{1}^{-1}$ (and their inverses),

- the (3)-essential monomials for $G_{2}$ are $x_{0} x_{1}^{-1} y_{0} y_{1}^{-1}$ and $x_{0} x_{1}^{-1} y_{0}^{-1} y_{1}$ (and their inverses).

Example 4.2.9 Let $W:=G_{4}$. The field of definition of $G_{4}$ is $\mathbb{Q}\left(\zeta_{3}\right)$. We have seen that

$$
\begin{array}{ll}
\mathcal{H}\left(G_{4}\right)=<S, T \mid S T S=T S T, & \left(S-u_{0}\right)\left(S-u_{1}\right)\left(S-u_{2}\right)=0, \\
& \left(T-u_{0}\right)\left(T-u_{1}\right)\left(T-u_{2}\right)=0>.
\end{array}
$$

Set $v_{0}^{6}:=u_{0}, v_{1}^{6}:=\zeta_{3}^{2} u_{1}, v_{2}^{6}:=\zeta_{3} u_{2}$. By Theorem 4.2.4, the algebra $\mathbb{Q}\left(\zeta_{3}\right)\left(v_{0}, v_{1}, v_{2}\right) \mathcal{H}\left(G_{4}\right)$ is split semisimple and hence, there exists a bijection between its irreducible characters and the irreducible characters of $G_{4}$. The group $G_{4}$ has 3 irreducible characters of degree 1, 3 irreducible characters of degree 2 and 1 irreducible character of degree 3. Set 


$$
\begin{gathered}
s_{1}\left(v_{0}, v_{1}, v_{2}\right)=\Phi_{9}^{\prime \prime}\left(v_{0} v_{1}^{-1}\right) \cdot \Phi_{18}^{\prime}\left(v_{0} v_{1}^{-1}\right) \cdot \Phi_{4}\left(v_{0} v_{1}^{-1}\right) \cdot \Phi_{12}^{\prime}\left(v_{0} v_{1}^{-1}\right) \cdot \Phi_{12}^{\prime \prime}\left(v_{0} v_{1}^{-1}\right) \cdot \Phi_{36}^{\prime}\left(v_{0} v_{1}^{-1}\right) \cdot \\
\Phi_{9}^{\prime}\left(v_{0} v_{2}^{-1}\right) \cdot \Phi_{18}^{\prime \prime}\left(v_{0} v_{2}^{-1}\right) \cdot \Phi_{4}\left(v_{0} v_{2}^{-1}\right) \cdot \Phi_{12}^{\prime}\left(v_{0} v_{2}^{-1}\right) \cdot \Phi_{12}^{\prime \prime}\left(v_{0} v_{2}^{-1}\right) \cdot \Phi_{36}^{\prime \prime}\left(v_{0} v_{2}^{-1}\right) \cdot \\
\Phi_{4}\left(v_{0}^{2} v_{1}^{-1} v_{2}^{-1}\right) \cdot \Phi_{12}^{\prime}\left(v_{0}^{2} v_{1}^{-1} v_{2}^{-1}\right) \cdot \Phi_{12}^{\prime \prime}\left(v_{0}^{2} v_{1}^{-1} v_{2}^{-1}\right), \\
s_{2}\left(v_{0}, v_{1}, v_{2}\right)=-\zeta_{3}^{2} v_{2}^{6} v_{1}^{-6} \cdot \Phi_{9}^{\prime}\left(v_{1} v_{0}^{-1}\right) \cdot \Phi_{18}^{\prime \prime}\left(v_{1} v_{0}^{-1}\right) \cdot \Phi_{9}^{\prime \prime}\left(v_{2} v_{0}^{-1}\right) \cdot \Phi_{18}^{\prime}\left(v_{2} v_{0}^{-1}\right) \cdot \Phi_{4}\left(v_{1} v_{2}^{-1}\right) \cdot \\
\Phi_{12}^{\prime}\left(v_{1} v_{2}^{-1}\right) \cdot \Phi_{12}^{\prime \prime}\left(v_{1} v_{2}^{-1}\right) \cdot \Phi_{36}^{\prime}\left(v_{1} v_{2}^{-1}\right) \cdot \Phi_{4}\left(v_{0}^{-2} v_{1} v_{2}\right) \cdot \Phi_{12}^{\prime}\left(v_{0}^{-2} v_{1} v_{2}\right) \cdot \Phi_{12}^{\prime \prime}\left(v_{0}^{-2} v_{1} v_{2}\right), \\
s_{3}\left(v_{0}, v_{1}, v_{2}\right)=\Phi_{4}\left(v_{0}^{2} v_{1}^{-1} v_{2}^{-1}\right) \cdot \Phi_{12}^{\prime}\left(v_{0}^{2} v_{1}^{-1} v_{2}^{-1}\right) \cdot \Phi_{12}^{\prime \prime}\left(v_{0}^{2} v_{1}^{-1} v_{2}^{-1}\right) \cdot \Phi_{4}\left(v_{1}^{2} v_{2}^{-1} v_{0}^{-1}\right) \cdot \Phi_{12}^{\prime}\left(v_{1}^{2} v_{2}^{-1} v_{0}^{-1}\right) \cdot \\
\Phi_{12}^{\prime \prime}\left(v_{1}^{2} v_{2}^{-1} v_{0}^{-1}\right) \cdot \Phi_{4}\left(v_{2}^{2} v_{0}^{-1} v_{1}^{-1}\right) \cdot \Phi_{12}^{\prime}\left(v_{2}^{2} v_{0}^{-1} v_{1}^{-1}\right) \cdot \Phi_{12}^{\prime \prime}\left(v_{2}^{2} v_{0}^{-1} v_{1}^{-1}\right),
\end{gathered}
$$

where $\Phi_{4}(x)=x^{2}+1, \Phi_{9}^{\prime}(x)=x^{3}-\zeta_{3}, \Phi_{9}^{\prime \prime}(x)=x^{3}-\zeta_{3}^{2}, \Phi_{12}^{\prime \prime}(x)=x^{2}+\zeta_{3}$, $\Phi_{12}^{\prime}(x)=x^{2}+\zeta_{3}^{2}, \Phi_{18}^{\prime \prime}(x)=x^{3}+\zeta_{3}, \Phi_{18}^{\prime}(x)=x^{3}+\zeta_{3}^{2}, \Phi_{36}^{\prime \prime}(x)=x^{6}+\zeta_{3}$, $\Phi_{36}^{\prime}(x)=x^{6}+\zeta_{3}^{2}$.

The Schur elements of $\mathcal{H}\left(G_{4}\right)$ are

$$
\begin{gathered}
s_{1}\left(v_{0}, v_{1}, v_{2}\right), s_{1}\left(v_{1}, v_{2}, v_{0}\right), s_{1}\left(v_{2}, v_{0}, v_{1}\right), \\
s_{2}\left(v_{0}, v_{1}, v_{2}\right), s_{2}\left(v_{1}, v_{2}, v_{0}\right), s_{2}\left(v_{2}, v_{0}, v_{1}\right), s_{3}\left(v_{0}, v_{1}, v_{2}\right) .
\end{gathered}
$$

We deduce that the (2)-essential monomials for $G_{4}$ are

$$
v_{0} v_{1}^{-1}, v_{0} v_{2}^{-1}, v_{1} v_{2}^{-1}, v_{0}^{2} v_{1}^{-1} v_{2}^{-1}, v_{1}^{2} v_{2}^{-1} v_{0}^{-1}, v_{2}^{2} v_{0}^{-1} v_{1}^{-1} .
$$

The first three are also the $\left(1-\zeta_{3}\right)$-essential monomials for $G_{4}$.

\subsection{Cyclotomic Hecke algebras}

Let $y$ be an indeterminate. We set $x:=y^{|\mu(K)|}$.

Definition 4.3.1 A cyclotomic specialization of $\mathcal{H}$ is a $\mathbb{Z}_{K}$-algebra morphism $\phi: \mathbb{Z}_{K}\left[\mathbf{v}, \mathbf{v}^{-1}\right] \rightarrow \mathbb{Z}_{K}\left[y, y^{-1}\right]$ with the following properties:

- $\phi: v_{\mathcal{C}, j} \mapsto y^{n_{\mathcal{C}, j}}$ where $n_{\mathcal{C}, j} \in \mathbb{Z}$ for all $\mathcal{C}$ and $j$.

- For all $\mathcal{C} \in \mathcal{A} / W$, if $z$ is another indeterminate, the element of $\mathbb{Z}_{K}\left[y, y^{-1}, z\right]$ defined by

$$
\Gamma_{\mathcal{C}}(y, z):=\prod_{j=0}^{e_{\mathcal{C}}-1}\left(z-\zeta_{e_{\mathcal{C}}}^{j} y^{n_{\mathcal{C}, j}}\right)
$$

is invariant by the action of $\operatorname{Gal}(K(y) / K(x))$. 
If $\phi$ is a cyclotomic specialization of $\mathcal{H}$, the corresponding cyclotomic Hecke algebra is the $\mathbb{Z}_{K}\left[y, y^{-1}\right]$-algebra, denoted by $\mathcal{H}_{\phi}$, which is obtained as the specialization of the $\mathbb{Z}_{K}\left[\mathbf{v}, \mathbf{v}^{-1}\right]$-algebra $\mathcal{H}$ via the morphism $\phi$. It also has a symmetrizing form $t_{\phi}$ defined as the specialization of the canonical form $t$.

Remark: Sometimes we describe the morphism $\phi$ by the formula

$$
u_{\mathcal{C}, j} \mapsto \zeta_{e_{\mathcal{C}}}^{j} x^{n_{\mathcal{C}, j}}
$$

If now we set $q:=\zeta x$ for some root of unity $\zeta \in \mu(K)$, then the cyclotomic specialization $\phi$ becomes a $\zeta$-cyclotomic specialization and $\mathcal{H}_{\phi}$ can be also considered over $\mathbb{Z}_{K}\left[q, q^{-1}\right]$.

Example 4.3.2 The "spetsial" cyclotomic Hecke algebra $\mathcal{H}_{q}^{s}(W)$ is the 1-cyclotomic algebra obtained by the specialization

$$
u_{\mathcal{C}, 0} \mapsto q, u_{\mathcal{C}, j} \mapsto \zeta_{e_{\mathcal{C}}}^{j} \text { for } 1 \leq j \leq e_{\mathcal{C}}-1, \text { for all } \mathcal{C} \in \mathcal{A} / W .
$$

For example,

$\mathcal{H}_{q}^{s}\left(G_{2}\right)=<S, T \mid S T S T S T=\operatorname{TSTSTS},(S-q)(S+1)=(T-q)(T+1)=0>$. and

$\mathcal{H}_{q}^{s}\left(G_{4}\right)=<S, T \mid S T S=T S T,(S-q)\left(S^{2}+S+1\right)=(T-q)\left(T^{2}+T+1\right)=0>$.

Set $A:=\mathbb{Z}_{K}\left[\mathbf{v}, \mathbf{v}^{-1}\right]$ and $\Omega:=\mathbb{Z}_{K}\left[y, y^{-1}\right]$. Let $\phi: A \rightarrow \Omega$ be a cyclotomic specialization such that $\phi\left(v_{\mathcal{C}, j}\right)=y^{n_{\mathcal{C}, j}}$ for all $\mathcal{C}, j$. Recall that, for $\alpha \in \mathbb{Z} \backslash\{0\}$, we denote by $I^{\alpha}: \Omega \rightarrow \Omega$ the monomorphism $y \mapsto y^{\alpha}$.

Theorem 4.3.3 Let $\phi: A \rightarrow \Omega$ be a cyclotomic specialization like above. Then there exist an adapted $\mathbb{Z}_{K}$-algebra morphism $\varphi: A \rightarrow \Omega$ and $\alpha \in \mathbb{Z} \backslash\{0\}$ such that

$$
\phi=I^{\alpha} \circ \varphi
$$

Proof: We set $d:=\operatorname{gcd}\left(n_{\mathcal{C}, j}\right)$ and consider the cyclotomic specialization $\varphi: v_{\mathcal{C}, j} \mapsto y^{n_{\mathcal{C}, j} / d}$. We have $\phi=I^{d} \circ \varphi$. Since $\operatorname{gcd}\left(n_{\mathcal{C}, j} / d\right)=1$, there exist $a_{\mathcal{C}, j} \in \mathbb{Z}$ such that

$$
\sum_{\mathcal{C}, j} a_{\mathcal{C}, j}\left(n_{\mathcal{C}, j} / d\right)=1
$$

We have $y=\varphi\left(\prod_{\mathcal{C}, j} v_{\mathcal{C}, j}^{a_{\mathcal{C}, j}}\right)$ an hence, $\varphi$ is surjective. Then, by Proposition 1.4.12, $\varphi$ is adapted. 
Let $\varphi$ be defined as in Theorem 4.3.3 and $\mathcal{H}_{\varphi}$ the corresponding cyclotomic Hecke algebra. Proposition 3.2.1 implies that the algebra $K(y) \mathcal{H}_{\varphi}$ is split semisimple. Due to Corollary 2.4.11 and the theorem above, we deduce that

Proposition 4.3.4 The algebra $K(y) \mathcal{H}_{\phi}$ is split semisimple.

For $y=1$, the algebra $K(y) \mathcal{H}_{\phi}$ specializes to the group algebra $K W$ (the form $t_{\phi}$ becoming the canonical form on the group algebra). Thus, by "Tits' deformation theorem", the specialization $v_{\mathcal{C}, j} \mapsto 1$ defines the following bijections

$$
\begin{array}{ccccc}
\operatorname{Irr}(K(\mathbf{v}) \mathcal{H}) & \leftrightarrow & \operatorname{Irr}\left(K(y) \mathcal{H}_{\phi}\right) & \leftrightarrow & \operatorname{Irr}(W) \\
\chi_{\mathbf{v}} & \mapsto & \chi_{\phi} & \mapsto & \chi
\end{array}
$$

The following result is an immediate consequence of Theorem 4.2.5,

Proposition 4.3.5 The Schur element $s_{\chi_{\phi}}(y)$ associated to the irreducible character $\chi_{\phi}$ of $K(y) \mathcal{H}_{\phi}$ is a Laurent polynomial in $y$ of the form

$$
s_{\chi_{\phi}}(y)=\psi_{\chi, \phi} y^{a_{\chi, \phi}} \prod_{\Phi \in C_{K}} \Phi(y)^{n_{\chi, \phi, \Phi}}
$$

where $\psi_{\chi, \phi} \in \mathbb{Z}_{K}, a_{\chi, \phi} \in \mathbb{Z}, n_{\chi, \phi, \Phi} \in \mathbb{N}$ and $C_{K}$ is a set of $K$-cyclotomic polynomials.

\subsubsection{Essential hyperplanes}

Let $\mathfrak{p}$ be a prime ideal of $\mathbb{Z}_{K}$. Let $\phi: v_{\mathcal{C}, j} \mapsto y^{n_{\mathcal{C}, j}}$ be a cyclotomic specialization of $\mathcal{H}$ and let $\varphi$ be an adapted morphism as in Theorem 4.3.3. By Corollary 3.4.2, the blocks of $\Omega_{\mathfrak{p} \Omega} \mathcal{H}_{\phi}$ coincide with the blocks of $\Omega_{\mathfrak{p} \Omega} \mathcal{H}_{\varphi}$ and the latter can be calculated with the use of Theorem 3.3.2. Therefore, we need to know which $\mathfrak{p}$-essential monomials are sent to 1 by $\varphi$.

Let $M:=\prod_{\mathcal{C}, j} v_{\mathcal{C}, j}^{a_{\mathcal{C}, j}}$ be a $\mathfrak{p}$-essential monomial for $W$. Then

$$
\varphi(M)=1 \Leftrightarrow \phi(M)=1 \Leftrightarrow \sum_{\mathcal{C}, j} a_{\mathcal{C}, j} n_{\mathcal{C}, j}=0 .
$$

Set $m:=\sum_{\mathcal{C} \in \mathcal{A} / W} e_{\mathcal{C}}$. The hyperplane defined in $\mathbb{C}^{m}$ by the relation

$$
\sum_{\mathcal{C}, j} a_{\mathcal{C}, j} t_{\mathcal{C}, j}=0
$$

where $\left(t_{\mathcal{C}, j}\right)_{\mathcal{C}, j}$ is a set of $m$ indeterminates, is called $\mathfrak{p}$-essential hyperplane for $W$. A hyperplane in $\mathbb{C}^{m}$ is called essential for $W$, if it is $\mathfrak{p}$-essential for some prime ideal $\mathfrak{p}$ of $\mathbb{Z}_{K}$. 
Example 4.3.6 Let $W:=G_{2}$. Following Example 4.2.8, let

$$
\phi: x_{0} \mapsto y^{n_{0}}, x_{1} \mapsto y^{n_{1}}, y_{0} \mapsto y^{m_{0}}, y_{1} \mapsto y^{m_{1}}
$$

be a cyclotomic specialization. Then

- the (2)-essential hyperplanes for $G_{2}$ are $N_{0}-N_{1}=0$ and $M_{0}-M_{1}=0$,

- the (3)-essential hyperplanes for $G_{2}$ are $N_{0}-N_{1}+M_{0}-M_{1}=0$ and $N_{0}-$ $N_{1}-M_{0}+M_{1}=0$.

Example 4.3.7 Let $W:=G_{4}$. Following Example 4.2.9, let $\phi: v_{i} \mapsto y^{n_{i}}$ for $i=0,1,2$ be a cyclotomic specialization. Then the hyperplanes

- $N_{0}-N_{1}=0, N_{0}-N_{2}=0$ and $N_{1}-N_{2}=0$ are (2)-essential and $\left(1-\zeta_{3}\right)$ essential for $G_{4}$,

- $2 N_{0}-N_{1}-N_{2}=0,2 N_{1}-N_{2}-N_{0}=0$ and $2 N_{2}-N_{0}-N_{1}=0$ are just (2)-essential for $G_{4}$.

In order to calculate the blocks of $\Omega_{\mathfrak{p} \Omega} \mathcal{H}_{\phi}$, we check to which $\mathfrak{p}$-essential hyperplanes the $n_{\mathcal{C}, j}$ belong and we apply Theorem 3.3.2:

- If the $n_{\mathcal{C}, j}$ belong to no $\mathfrak{p}$-essential hyperplane, then the blocks of $\Omega_{\mathfrak{p} \Omega} \mathcal{H}_{\phi}$ coincide with the blocks of $A_{\mathfrak{p} A} \mathcal{H}$. We call these blocks $\mathfrak{p}$-blocks associated with no essential hyperplane.

- If the $n_{\mathcal{C}, j}$ belong to exactly one $\mathfrak{p}$-essential hyperplane $H_{M}$, corresponding to the $\mathfrak{p}$-essential monomial $M$, then the blocks of $\Omega_{\mathfrak{p} \Omega} \mathcal{H}_{\phi}$ coincide with the blocks of $A_{\mathfrak{q}_{M}} \mathcal{H}$, where $\mathfrak{q}_{M}:=\mathfrak{p} A+(M-1) A$. We call these blocks $\mathfrak{p}$-blocks associated with the essential hyperplane $H_{M}$.

- If the $n_{\mathcal{C}, j}$ belong to more than one $\mathfrak{p}$-essential hyperplane, then, following Theorem 3.3.2, the blocks of $\Omega_{\mathfrak{p} \Omega} \mathcal{H}_{\phi}$ are unions of the $\mathfrak{p}$-blocks associated with the $\mathfrak{p}$-essential hyperplanes to which the $n_{\mathcal{C}, j}$ belong and they are minimal with respect to that property.

This last property of the $\mathfrak{p}$-blocks is called "property of semi-continuity" (the name is due to C. Bonnafé). The property of semi-continuity also appears in works on Kazhdan-Lusztig cells (cf. [10], [9], [39]) and on Cherednik algebras (cf. [37]). In the next section, we will see that the Rouquier blocks of the cyclotomic Hecke algebras also have this property. 


\subsubsection{Group algebra}

Let $\mathfrak{p}$ be a prime ideal of $\mathbb{Z}_{K}$ lying over a prime number $p$ and let $\phi: v_{\mathcal{C}, j} \mapsto$ $y^{n_{\mathcal{C}, j}}$ be a cyclotomic specialization of $\mathcal{H}$. If $n_{\mathcal{C}, j}=n \in \mathbb{Z}$ for all $\mathcal{C}$ and $j$, then the $n_{\mathcal{C}, j}$ belong to all $\mathfrak{p}$-essential hyperplanes for $W$ and we have $\Omega_{\mathfrak{p} \Omega} \mathcal{H}_{\phi} \cong \Omega_{\mathfrak{p} \Omega} W$. Note that, since the ring $\Omega_{\mathfrak{p} \Omega}$ is a discrete valuation ring (by Theorem 1.2.24), the blocks of $\Omega_{\mathfrak{p} \Omega} W$ are the $p$-blocks of $W$ as determined by Brauer theory. Due to Theorem 3.3.2, we obtain the following result which relates the $\mathfrak{p}$-blocks of any cyclotomic Hecke algebra to the $p$-blocks of $W$.

Proposition 4.3.8 Let $\phi: v_{\mathcal{C}, j} \mapsto y^{n_{\mathcal{C}, j}}$ be a cyclotomic specialization of $\mathcal{H}$. If two irreducible characters $\chi, \psi \in \operatorname{Irr}(W)$ are in the same block of $\Omega_{p \Omega} \mathcal{H}_{\phi}$, then they are in the same p-block of $W$.

Proof: The blocks of $\Omega_{\mathfrak{p} \Omega} H_{\phi}$ are unions of the blocks of $A_{\mathfrak{q}_{M}} \mathcal{H}$ for all p-essential monomials $M$ such that $\phi(M)=1$, whereas the $p$-blocks of $W$ are unions of the blocks of $A_{\mathfrak{q}_{M}} \mathcal{H}$ for all $\mathfrak{p}$-essential monomials $M$.

However, we know from Brauer theory that if the order of the group $W$ is prime to $p$, then every character of $W$ is a $p$-block by itself (see, for example, [59], 15.5, Proposition 43). It is an immediate consequence of Proposition 4.3 .8 that

Corollary 4.3.9 If $\mathfrak{p}$ is a prime ideal of $\mathbb{Z}_{K}$ lying over a prime number $p$ which does not divide the order of the group $W$, then the blocks of $\Omega_{p \Omega} \mathcal{H}_{\phi}$ are singletons.

\subsection{Rouquier blocks of the cyclotomic Hecke algebras}

Definition 4.4.1 We call Rouquier ring of $K$ and denote by $\mathcal{R}_{K}(y)$ the $\mathbb{Z}_{K^{-}}$ subalgebra of $K(y)$

$$
\mathcal{R}_{K}(y):=\mathbb{Z}_{K}\left[y, y^{-1},\left(y^{n}-1\right)_{n \geq 1}^{-1}\right]
$$

Let $\phi: v_{\mathcal{C}, j} \mapsto y^{n_{\mathcal{C}, j}}$ be a cyclotomic specialization and $\mathcal{H}_{\phi}$ the corresponding cyclotomic Hecke algebra. The Rouquier blocks of $\mathcal{H}_{\phi}$ are the blocks of the algebra $\mathcal{R}_{K}(y) \mathcal{H}_{\phi}$.

It has been shown by Rouquier (cf. [57]), that if $W$ is a Weyl group and $\mathcal{H}_{\phi}$ is obtained via the "spetsial" cyclotomic specialization (see Example 
4.3.2), then the Rouquier blocks of $\mathcal{H}_{\phi}$ coincide with the families of characters defined by Lusztig. Thus, the Rouquier blocks generalize the notion of "families of characters" to all complex reflection groups.

Remark: We have seen that if we set $q:=\zeta y^{|\mu(K)|}$ for some root of unity $\zeta \in \mu(K)$, then the cyclotomic Hecke algebra $\mathcal{H}_{\phi}$ can be also considered over the ring $\mathbb{Z}_{K}\left[q, q^{-1}\right]$. We define the Rouquier blocks of $\mathcal{H}_{\phi}$ to be the blocks of $\mathcal{R}_{K}(y) \mathcal{H}_{\phi}$. However, in other texts, as, for example, in [18], the Rouquier blocks are defined to be the blocks of $\mathcal{R}_{K}(q) \mathcal{H}_{\phi}$. Since $\mathcal{R}_{K}(y)$ is the integral closure of $\mathcal{R}_{K}(q)$ in the splitting field $K(y)$ for $\mathcal{H}_{\phi}$, Proposition 2.1.9 establishes the (determining) relation between the blocks of $\mathcal{R}_{K}(y) \mathcal{H}_{\phi}$ and the blocks of $\mathcal{R}_{K}(q) \mathcal{H}_{\phi}$.

The Rouquier ring $\mathcal{R}_{K}(y)$ has many interesting properties. The next result describes some of them.

\section{Proposition 4.4.2}

1. The group of units $\mathcal{R}_{K}(y)^{\times}$of the Rouquier ring $\mathcal{R}_{K}(y)$ consists of the elements of the form

$$
u y^{n} \prod_{\Phi \in \operatorname{Cycl}(K)} \Phi(y)^{n_{\Phi}},
$$

where $u \in \mathbb{Z}_{K}^{\times}, n, n_{\Phi} \in \mathbb{Z}, \operatorname{Cycl}(K)$ is the set of $K$-cyclotomic polynomials and $n_{\Phi}=0$ for all but a finite number of $\Phi$.

2. The prime ideals of $\mathcal{R}_{K}(y)$ are

- the zero ideal $\{0\}$,

- the ideals of the form $\mathfrak{p} \mathcal{R}_{K}(y)$, where $\mathfrak{p}$ is a prime ideal of $\mathbb{Z}_{K}$,

- the ideals of the form $P(y) \mathcal{R}_{K}(y)$, where $P(y)$ is an irreducible element of $\mathbb{Z}_{K}[y]$ of degree at least 1 , prime to $y$ and to $\Phi(y)$ for all $\Phi \in \operatorname{Cycl}(K)$.

3. The Rouquier ring $\mathcal{R}_{K}(y)$ is a Dedekind ring.

\section{Proof:}

1. This part is immediate from the definition of $K$-cyclotomic polynomials. 
2. Since $\mathcal{R}_{K}(y)$ is an integral domain, the zero ideal is prime.

The ring $\mathbb{Z}_{K}$ is a Dedekind ring and thus a Krull ring, by Proposition 1.2.26. Proposition 1.2.25 implies that the ring $\mathbb{Z}_{K}[y]$ is also a Krull ring whose prime ideals of height 1 are of the form $\mathfrak{p} \mathbb{Z}_{K}[y]$ (p prime in $\left.\mathbb{Z}_{K}\right)$ and $P(y) \mathbb{Z}_{K}[y]\left(P(y)\right.$ irreducible in $\mathbb{Z}_{K}[y]$ of degree at least 1$)$. Moreover, $\mathbb{Z}_{K}$ has an infinite number of non-zero prime ideals whose intersection is the zero ideal. Since all non-zero prime ideals of $\mathbb{Z}_{K}$ are maximal, we obtain that every prime ideal of $\mathbb{Z}_{K}$ is the intersection of maximal ideals. Thus $\mathbb{Z}_{K}$ is, by definition, a Jacobson ring (cf. [30], $\S 4.5)$. The general form of the Nullstellensatz ([30], Theorem 4.19) implies that for every maximal ideal $\mathfrak{m}$ of $\mathbb{Z}_{K}[y]$, the ideal $\mathfrak{m} \cap \mathbb{Z}_{K}$ is a maximal ideal of $\mathbb{Z}_{K}$. We deduce that the maximal ideals of $\mathbb{Z}_{K}[y]$ are of the form $\mathfrak{p} \mathbb{Z}_{K}[y]+P(y) \mathbb{Z}_{K}[y]$ (p prime in $\mathbb{Z}_{K}$ and $P(y)$ of degree at least 1 irreducible modulo $\mathfrak{p})$. Since $\mathbb{Z}_{K}[y]$ has Krull dimension 2, we have now described all its prime ideals.

The Rouquier ring $\mathcal{R}_{K}(y)$ is a localization of $\mathbb{Z}_{K}[y]$. Therefore, in order to prove that the non-zero prime ideals of $\mathcal{R}_{K}(y)$ are the ones described above, it is enough to show that $\mathfrak{m} \mathcal{R}_{K}(y)=\mathcal{R}_{K}(y)$ for all maximal ideals $\mathfrak{m}$ of $\mathbb{Z}_{K}[y]$. For this, it suffices to show that $\mathfrak{p} \mathcal{R}_{K}(y)$ is a maximal ideal of $\mathcal{R}_{K}(y)$ for all prime ideals $\mathfrak{p}$ of $\mathbb{Z}_{K}$.

Let $\mathfrak{p}$ be a prime ideal of $\mathbb{Z}_{K}$. Then

$$
\mathcal{R}_{K}(y) / \mathfrak{p} \mathcal{R}_{K}(y) \cong \mathbb{F}_{\mathfrak{p}}\left[y, y^{-1},\left(y^{n}-1\right)_{n \geq 1}^{-1}\right],
$$

where $\mathbb{F}_{\mathfrak{p}}$ denotes the finite field $\mathbb{Z}_{K} / \mathfrak{p}$. Since $\mathbb{F}_{\mathfrak{p}}$ is finite, every non-zero polynomial in $\mathbb{F}_{\mathfrak{p}}[y]$ is a product of elements which divide $y$ or $y^{n}-1$ for some $n \in \mathbb{N}$. Thus every non-zero element of $\mathbb{F}_{\mathfrak{p}}[y]$ is invertible in $\mathcal{R}_{K}(y) / \mathfrak{p} \mathcal{R}_{K}(y)$. Consequently, we obtain that

$$
\mathcal{R}_{K}(y) / \mathfrak{p} \mathcal{R}_{K}(y) \cong \mathbb{F}_{\mathfrak{p}}(y),
$$

whence $\mathfrak{p}$ generates a maximal ideal in $\mathcal{R}_{K}(y)$.

3. The ring $\mathcal{R}_{K}(y)$ is the localization of a Noetherian integrally closed ring and thus Noetherian and integrally closed itself. Moreover, following the description of its prime ideals in part 2, it has Krull dimension 1.

Remark: If $P(y)$ is an irreducible element of $\mathbb{Z}_{K}[y]$ of degree at least 1 , prime to $y$ and to $\Phi(y)$ for all $\Phi \in \operatorname{Cycl}(K)$, then the field $\mathcal{R}_{K}(y) / P(y) \mathcal{R}_{K}(y)$ is isomorphic to the field of fractions of the ring $\mathbb{Z}_{K}[y] / P(y) \mathbb{Z}_{K}[y]$. 
Now let us recall the form of the Schur elements of the cyclotomic Hecke algebra $\mathcal{H}_{\phi}$ given in Proposition 4.3.5. If $\chi_{\phi}$ is an irreducible character of $K(y) \mathcal{H}_{\phi}$, then its Schur element $s_{\chi_{\phi}}(y)$ is of the form

$$
s_{\chi_{\phi}}(y)=\psi_{\chi, \phi} y^{a_{\chi, \phi}} \prod_{\Phi \in C_{K}} \Phi(y)^{n_{\chi, \phi, \Phi}}
$$

where $\psi_{\chi, \phi} \in \mathbb{Z}_{K}, a_{\chi, \phi} \in \mathbb{Z}, n_{\chi, \phi, \Phi} \in \mathbb{N}$ and $C_{K}$ is a set of $K$-cyclotomic polynomials.

Definition 4.4.3 A prime ideal $\mathfrak{p}$ of $\mathbb{Z}_{K}$ lying over a prime number $p$ is $\phi$-bad for $W$, if there exists $\chi_{\phi} \in \operatorname{Irr}\left(K(y) \mathcal{H}_{\phi}\right)$ with $\psi_{\chi, \phi} \in \mathfrak{p}$. If $\mathfrak{p}$ is $\phi$-bad for $W$, we say that $p$ is a $\phi$-bad prime number for $W$.

Remark: If $W$ is a Weyl group and $\phi$ is the "spetsial" cyclotomic specialization, then the $\phi$-bad prime ideals are the ideals generated by the bad prime numbers (in the "usual" sense) for $W$ (see [34], 5.2).

Note that if $p$ is a $\phi$-bad prime number for $W$, then $p$ must divide the order of the group (since $\left.s_{\chi_{\phi}}(1)=|W| / \chi(1)\right)$.

Let us denote by $\mathcal{O}$ the Rouquier ring. By Proposition 2.1.10, the Rouquier blocks of $\mathcal{H}_{\phi}$ are unions of the blocks of $\mathcal{O}_{\mathcal{P}} \mathcal{H}_{\phi}$, where $\mathcal{P}$ runs over the set of prime ideals of $\mathcal{O}$. However, in all of the following cases, due to the form of the Schur elements, the blocks of $\mathcal{O}_{\mathcal{P}} \mathcal{H}_{\phi}$ are singletons (i.e., $e_{\chi_{\phi}}=\chi_{\phi}^{\vee} / s_{\chi_{\phi}} \in \mathcal{O}_{\mathcal{P}} \mathcal{H}_{\phi}$ for all $\left.\chi_{\phi} \in \operatorname{Irr}\left(K(y) \mathcal{H}_{\phi}\right)\right)$ :

- $\mathcal{P}$ is the zero ideal $\{0\}$.

- $\mathcal{P}$ is of the form $P(y) \mathcal{O}$, where $P(y)$ is an irreducible element of $\mathbb{Z}_{K}[y]$ of degree at least 1, prime to $y$ and to $\Phi(y)$ for all $\Phi \in \operatorname{Cycl}(K)$.

- $\mathcal{P}$ is of the form $\mathfrak{p O}$, where $\mathfrak{p}$ is a prime ideal of $\mathbb{Z}_{K}$ which is not $\phi$-bad for $W$.

Therefore, the blocks of $\mathcal{O H}_{\phi}$ are, simply, unions of the blocks of $\mathcal{O}_{\mathfrak{p O}} \mathcal{H}_{\phi}$, where $\mathfrak{p}$ runs over the set of $\phi$-bad prime ideals $\mathfrak{p}$ of $\mathbb{Z}_{K}$. More precisely, we have that

Proposition 4.4.4 Let $\chi, \psi \in \operatorname{Irr}(W)$. The characters $\chi_{\phi}$ and $\psi_{\phi}$ are in the same Rouquier block of $\mathcal{H}_{\phi}$ if and only if there exist a finite sequence $\chi_{0}, \chi_{1}, \ldots, \chi_{n} \in \operatorname{Irr}(W)$ and a finite sequence $\mathfrak{p}_{1}, \ldots, \mathfrak{p}_{n}$ of $\phi$-bad prime ideals for $W$ such that 
- $\left(\chi_{0}\right)_{\phi}=\chi_{\phi}$ and $\left(\chi_{n}\right)_{\phi}=\psi_{\phi}$,

- for all $j(1 \leq j \leq n), \quad\left(\chi_{j-1}\right)_{\phi}$ and $\left(\chi_{j}\right)_{\phi}$ are in the same block of $\mathcal{O}_{\mathfrak{p}_{j} \mathcal{O}} \mathcal{H}_{\phi}$

By Proposition 1.1.5 (4), we obtain that $\mathcal{O}_{\mathfrak{p O}} \cong \Omega_{\mathfrak{p} \Omega}$, where $\Omega:=\mathbb{Z}_{K}\left[y, y^{-1}\right]$. In the previous section we saw how we can use Theorem 3.3.2 to calculate the blocks of $\Omega_{p \Omega} \mathcal{H}_{\phi}$ and thus obtain the Rouquier blocks of $\mathcal{H}_{\phi}$. We deduce that the Rouquier blocks of the cyclotomic Hecke algebras also have the property of semi-continuity:

- If the $n_{\mathcal{C}, j}$ belong to no essential hyperplane for $W$, then the Rouquier blocks of $\mathcal{H}_{\phi}$ are the Rouquier blocks associated with no essential hyperplane.

- If the $n_{\mathcal{C}, j}$ belong to exactly one essential hyperplane $H$ for $W$, then the Rouquier blocks of $\mathcal{H}_{\phi}$ are the Rouquier blocks associated with the essential hyperplane $H$.

- If the $n_{\mathcal{C}, j}$ belong to more than one essential hyperplane, then the Rouquier blocks of $\mathcal{H}_{\phi}$ are unions of the Rouquier blocks associated with the essential hyperplanes to which the $n_{\mathcal{C}, j}$ belong and they are minimal with respect to that property.

\subsubsection{Rouquier blocks and central morphisms}

The following description of the Rouquier blocks results from Proposition 2.1 .15 and the description of $\phi$-bad prime ideals for $W$.

Proposition 4.4.5 Let $\chi, \psi \in \operatorname{Irr}(W)$. The characters $\chi_{\phi}$ and $\psi_{\phi}$ are in the same Rouquier block of $\mathcal{H}_{\phi}$ if and only if there exists a finite sequence $\chi_{0}, \chi_{1}, \ldots, \chi_{n} \in \operatorname{Irr}(W)$ and a finite sequence $\mathfrak{p}_{1}, \ldots, \mathfrak{p}_{n}$ of $\phi$-bad prime ideals for $W$ such that

- $\left(\chi_{0}\right)_{\phi}=\chi_{\phi}$ and $\left(\chi_{n}\right)_{\phi}=\psi_{\phi}$,

- for all $j(1 \leq j \leq n), \omega_{\left(\chi_{j-1}\right)_{\phi}} \equiv \omega_{\left(\chi_{j}\right)_{\phi}} \bmod \mathfrak{p}_{j} \mathcal{O}_{\mathfrak{p}_{j} \mathcal{O}}$.

\subsubsection{Rouquier blocks and functions $a$ and $A$}

Following the notations of [20], $6 \mathrm{~B}$, for every element $P(y) \in \mathbb{C}(y)$, we call

- valuation of $P(y)$ at $y$ and denote by $\operatorname{val}_{y}(P)$ the order of $P(y)$ at 0 (we have $\operatorname{val}_{y}(P)<0$ if 0 is a pole of $P(y)$ and $\operatorname{val}_{y}(P)>0$ if 0 is a zero of $P(y))$, 
- degree of $P(y)$ at $y$ and denote by $\operatorname{deg}_{y}(P)$ the opposite of the valuation of $P(1 / y)$.

Moreover, if $x:=y^{|\mu(K)|}$, then

$$
\operatorname{val}_{x}(P(y)):=\frac{\operatorname{val}_{y}(P)}{|\mu(K)|} \text { and } \operatorname{deg}_{x}(P(y)):=\frac{\operatorname{deg}_{y}(P)}{|\mu(K)|} .
$$

For $\chi \in \operatorname{Irr}(W)$, we define

$$
a_{\chi_{\phi}}:=\operatorname{val}_{x}\left(s_{\chi_{\phi}}(y)\right) \text { and } A_{\chi_{\phi}}:=\operatorname{deg}_{x}\left(s_{\chi_{\phi}}(y)\right) .
$$

The following result is proven in [18, Proposition 2.9.

\section{Proposition 4.4.6}

1. For all $\chi \in \operatorname{Irr}(W)$, we have

$$
\omega_{\chi_{\phi}}(\tau)=t_{\phi}(\tau) x^{a_{\chi_{\phi}}+A_{\chi_{\phi}}},
$$

where $\tau$ is the central element of the pure braid group defined in 4.1 .6 .

2. Let $\chi, \psi \in \operatorname{Irr}(W)$. If $\chi_{\phi}$ and $\psi_{\phi}$ belong to the same Rouquier block, then

$$
a_{\chi_{\phi}}+A_{\chi_{\phi}}=a_{\psi_{\phi}}+A_{\psi_{\phi}}
$$

\section{Proof:}

1. If $P(y) \in \mathbb{C}\left[y, y^{-1}\right]$, we denote by $P(y)^{*}$ the polynomial whose coefficients are the complex conjugates of those of $P(y)$. By [20], 2.8, we know that the Schur element $s_{\chi_{\phi}}(y)$ is semi-palindromic and satisfies

$$
s_{\chi_{\phi}}\left(y^{-1}\right)^{*}=\frac{t_{\phi}(\tau)}{\omega_{\chi_{\phi}}(\tau)} s_{\chi_{\phi}}(y) .
$$

We deduce $([20], 6.5,6.6)$ that

$$
\frac{t_{\phi}(\tau)}{\omega_{\chi_{\phi}}(\tau)}=\xi x^{-\left(a_{\chi_{\phi}}+A_{\chi_{\phi}}\right)},
$$

for some $\xi \in \mathbb{C}$. For $y=x=1$, the first equation gives $t_{\phi}(\tau)=\omega_{\chi_{\phi}}(\tau)$ and the second one $\xi=1$. Thus we obtain

$$
\omega_{\chi_{\phi}}(\tau)=t_{\phi}(\tau) x^{a_{\chi_{\phi}}+A_{\chi_{\phi}}} .
$$


2. Suppose that $\chi_{\phi}$ and $\psi_{\phi}$ belong to the same Rouquier block. Due to Proposition 4.4.5, it is enough to show that if there exists a $\phi$-bad prime ideal $\mathfrak{p}$ of $\mathbb{Z}_{K}$ such that $\omega_{\chi_{\phi}} \equiv \omega_{\psi_{\phi}} \bmod \mathfrak{p} \mathcal{O}_{\mathfrak{p O}}$, then $a_{\chi_{\phi}}+A_{\chi_{\phi}}=$ $a_{\psi_{\phi}}+A_{\psi_{\phi}}$. If $\omega_{\chi_{\phi}} \equiv \omega_{\psi_{\phi}} \bmod \mathfrak{p} \mathcal{O}_{\mathfrak{p O}}$, then, in particular, $\omega_{\chi_{\phi}}(\tau) \equiv$ $\omega_{\psi_{\phi}}(\tau) \bmod \mathfrak{p} \mathcal{O}_{\mathfrak{p} \mathcal{O}}$. Part 1 implies that

$$
t_{\phi}(\tau) x^{a_{\chi_{\phi}}+A_{\chi_{\phi}}} \equiv t_{\phi}(\tau) x^{a_{\psi_{\phi}}+A_{\psi_{\phi}}} \bmod \mathfrak{p} \mathcal{O}_{\mathfrak{p} \mathcal{O}}
$$

We know by [20], 2.1 that $t_{\phi}(\tau)$ is of the form $\xi x^{M}$, where $\xi$ is a root of unity and $M \in \mathbb{Z}$. Thus $t_{\phi}(\tau) \notin \mathfrak{p} \mathcal{O}_{\mathfrak{p O}}$ and the above congruence gives

$$
x^{a_{\chi_{\phi}}+A_{\chi_{\phi}}} \equiv x^{a_{\psi_{\phi}}+A_{\psi_{\phi}}} \bmod \mathfrak{p} \mathcal{O}_{\mathfrak{p O}}
$$

whence

$$
a_{\chi_{\phi}}+A_{\chi_{\phi}}=a_{\psi_{\phi}}+A_{\psi_{\phi}}
$$

Remark: For all Coxeter groups, Lusztig has proved (cf., for example, [45], 3.3 and 3.4) that if $\chi_{\phi}$ and $\psi_{\phi}$ belong to the same Rouquier block of the Iwahori-Hecke algebra, then $a_{\chi_{\phi}}=a_{\psi_{\phi}}$ and $A_{\chi_{\phi}}=A_{\psi_{\phi}}$. This assertion has also been proved

- for almost all cyclotomic Hecke algebras of the groups $G(d, 1, r)$ and $G(e, e, r)$ in [18],

- for the "spetsial" cyclotomic Hecke algebra of the "spetsial" exceptional complex reflection groups in [52].

Using the results of the next chapter, we have been able to obtain the same result for all cyclotomic Hecke algebras

- of the groups $G(d, 1, r)$ in [24],

- of the groups $G(d e, e, r)$ in [25], and

- of all exceptional irreducible complex reflection groups in [22],

thus completing its proof for all complex reflection groups. 


\section{Chapter 5}

\section{On the determination of the Rouquier blocks}

The aim of this chapter is the determination of the Rouquier blocks of the cyclotomic Hecke algebras of all irreducible complex reflection groups. In the last chapter, we saw that the Rouquier blocks have the property of "semicontinuity". This property allows us to obtain the Rouquier blocks for any cyclotomic Hecke algebra by actually calculating them in a small number of cases. Following the theory developed in the two previous chapters, we only need to determine the Rouquier blocks "associated with no and each essential hyperplane" for all irreducible complex reflection groups.

For the exceptional irreducible complex reflection groups, the computations were made with the use of the GAP package CHEVIE (cf. [36]). In section 5.2, we give the algorithm which has been used. This algorithm is heuristic and was applied only to the groups $G_{7}, G_{11}, G_{19}, G_{26}, G_{28}$ and $G_{32}$. The results presented in the Appendix allow us to use Clifford Theory in order to obtain the Rouquier blocks for the groups $G_{4}, \ldots, G_{22}$ and $G_{25}$. The remaining groups have already been studied by Malle and Rouquier in [52]. We have stored all the calculated data in a computer file and created GAP functions to display them. These functions are presented in this chapter and can be found on my webpage ([23]).

As far as the groups of the infinite series are concerned, Clifford theory again allows us to obtain the Rouquier blocks of the cyclotomic Hecke algebras of $G(d e, e, r)$ (when $r>2$ or $r=2$ and $e$ is odd) and $G(2 f d, 2 f, 2)$ from those of $G(d e, 1, r)$ and $G(2 f d, 2,2)$ respectively. Therefore, only the last two cases need to be studied thoroughly.

In section 5.3, we determine the Rouquier blocks associated with the essential hyperplanes for the group $G(d, 1, r)$. The algorithm of Lyle and Mathas (cf. [47]) for the determination of the blocks of an Ariki-Koike alge- 
bra over a field has played a key role in the achievement of this goal. The description of the Rouquier blocks for $G(d, 1, r)$ is combinatorial and demonstrates an unexpected relation between them and the families of characters of the Weyl groups of type $B_{n}, n \leq r$.

In section 5.4, we calculate the Rouquier blocks associated with no and each essential hyperplane for the group $G(2 d, 2,2)$. The method used follows the same principles as the algorithm for the exceptional irreducible complex reflection groups.

Finally, in section 5.5, we explain how exactly we apply the results of Clifford theory (Propositions 2.3.15 and 2.3.18) to obtain the Rouquier blocks of the cyclotomic Hecke algebras of the groups $G(d e, e, r)$.

\subsection{General principles}

Let $W$ be an irreducible complex reflection group with field of definition $K$ and let $\mathcal{H}$ be its generic Hecke algebra. We suppose that the assumptions 4.2.3 are satisfied. Following Theorem 4.2.4, we can find a set of indeterminates $\mathbf{v}$ such that the algebra $K(\mathbf{v}) \mathcal{H}$ is split semisimple. Set $A:=\mathbb{Z}_{K}\left[\mathbf{v}, \mathbf{v}^{-1}\right]$ and let us denote by $\mathcal{O}$ the Rouquier ring $\mathcal{R}_{K}(y)$ of $K$. Let $\mathfrak{p}$ be a prime ideal of $\mathbb{Z}_{K}$ lying over a prime number $p$ which divides the order of the group $W$. We can determine the $\mathfrak{p}$-essential hyperplanes $W$ from the factorization of the Schur elements of $\mathcal{H}$ over $K\left[\mathbf{v}, \mathbf{v}^{-1}\right]$.

Let $\phi_{\emptyset}: v_{\mathcal{C}, j} \mapsto y^{n_{\mathcal{C}, j}}$ be a cyclotomic specialization such that the integers $n_{\mathcal{C}, j}$ belong to no essential hyperplane for $W$. Such a cyclotomic specialization will be called associated with no essential hyperplane. The blocks of $\mathcal{O}_{\mathfrak{p O}} \mathcal{H}_{\phi_{\emptyset}}$ are called $\mathfrak{p}$-blocks associated with no essential hyperplane and coincide with the blocks of $A_{\mathfrak{p} A} \mathcal{H}$.

Let $\phi_{H}: v_{\mathcal{C}, j} \mapsto y^{n_{\mathcal{C}, j}}$ be a cyclotomic specialization such that the integers $n_{\mathcal{C}, j}$ belong to exactly one essential hyperplane $H$, corresponding to the essential monomial $M$. Such a cyclotomic specialization will be called associated with the essential hyperplane $H$. The blocks of $\mathcal{O}_{\mathfrak{p O}} \mathcal{H}_{\phi_{H}}$ are called $\mathfrak{p}$-blocks associated with the essential hyperplane $H$. If $H$ is not $\mathfrak{p}$-essential for $W$, then the blocks of $\mathcal{O}_{\mathfrak{p O}} \mathcal{H}_{\phi_{H}}$ coincide with the $\mathfrak{p}$-blocks associated with no essential hyperplane. If $H$ is $\mathfrak{p}$-essential for $W$, then the blocks of $\mathcal{O}_{\mathfrak{p O}} \mathcal{H}_{\phi_{H}}$ coincide with the blocks of $A_{\mathfrak{q}_{M}} \mathcal{H}$, where $\mathfrak{q}_{M}=(M-1) A+\mathfrak{p} A$. By Proposition 3.2.3, the $\mathfrak{p}$-blocks associated with the essential hyperplane $H$ are unions of $\mathfrak{p}$-blocks associated with no essential hyperplane.

Following Proposition 4.4.4, the Rouquier blocks of $\mathcal{H}_{\phi_{\emptyset}}$ can be obtained as unions of $\mathfrak{p}$-blocks associated with no essential hyperplane, where $\mathfrak{p}$ runs over the set of prime ideals of $\mathbb{Z}_{K}$ lying over the prime divisors of $|W|$ (if 
$\mathfrak{p}$ is not $\phi_{\emptyset}$-bad, then the corresponding $\mathfrak{p}$-blocks are trivial). The Rouquier blocks of $\mathcal{H}_{\phi_{\emptyset}}$ are the Rouquier blocks associated with no essential hyperplane. Respectively, the Rouquier blocks of $\mathcal{H}_{\phi_{H}}$ are the Rouquier blocks associated with the essential hyperplane $H$. Like the $\mathfrak{p}$-blocks, the Rouquier blocks associated with the essential hyperplane $H$ are unions of Rouquier blocks associated with no essential hyperplane.

The following result is a consequence of Theorem 3.3.2 and summarizes the results of Chapter 4 .

Theorem 5.1.1 Let $\phi: v_{\mathcal{C}, j} \mapsto y^{n_{\mathcal{C}, j}}$ be a cyclotomic specialization which is not associated with no essential hyperplane. Let $\mathcal{E}$ be the set of all essential hyperplanes to which the integers $n_{\mathcal{C}, j}$ belong. Let $\chi, \psi \in \operatorname{Irr}(W)$. The characters $\chi_{\phi}$ and $\psi_{\phi}$ belong to the same block of $\mathcal{O}_{\mathfrak{p O}} \mathcal{H}_{\phi}$ if and only if there exist a finite sequence $\chi_{0}, \chi_{1}, \ldots, \chi_{n} \in \operatorname{Irr}(W)$ and a finite sequence $H_{1}, \ldots, H_{n} \in \mathcal{E}$ such that

- $\left(\chi_{0}\right)_{\phi}=\chi_{\phi}$ and $\left(\chi_{n}\right)_{\phi}=\psi_{\phi}$,

- for all $j(1 \leq j \leq n), \quad\left(\chi_{j-1}\right)_{\phi}$ and $\left(\chi_{j}\right)_{\phi}$ are in the same $\mathfrak{p}$-block associated with the essential hyperplane $H_{j}$.

Moreover, the characters $\chi_{\phi}$ and $\psi_{\phi}$ belong to the same Rouquier block of $\mathcal{H}_{\phi}$ if and only if there exist a finite sequence $\chi_{0}, \chi_{1}, \ldots, \chi_{n} \in \operatorname{Irr}(W)$ and a finite sequence $H_{1}, \ldots, H_{n} \in \mathcal{E}$ such that

- $\left(\chi_{0}\right)_{\phi}=\chi_{\phi}$ and $\left(\chi_{n}\right)_{\phi}=\psi_{\phi}$,

- for all $j(1 \leq j \leq n)$, $\left(\chi_{j-1}\right)_{\phi}$ and $\left(\chi_{j}\right)_{\phi}$ are in the same Rouquier block associated with the essential hyperplane $H_{j}$.

Thanks to the above theorem, in order to determine the Rouquier blocks of any cyclotomic Hecke algebra associated to $W$, we only need to consider a cyclotomic specialization associated with no and each essential hyperplane and

- either calculate their $\mathfrak{p}$-blocks, for all prime ideals $\mathfrak{p}$ lying over the prime divisors of $|W|$, and use Proposition 4.4.4 in order to obtain their Rouquier blocks,

- or calculate directly their Rouquier blocks.

In the case of the exceptional groups, we will use the first method, whereas in the case of the groups of the infinite series, we will mostly use the second one. In both cases, we will need some criteria in order to determine 
the corresponding partitions of $\operatorname{Irr}(W)$ into blocks. These are results which have already been presented in previous chapters, but we are going to repeat here for the convenience of the reader. Once more, let $\phi: v_{\mathcal{C}, j} \mapsto y^{n_{\mathcal{C}, j}}$ be a cyclotomic specialization and let $\mathfrak{p}$ be a prime ideal of $\mathbb{Z}_{K}$ lying over the prime number $p$.

Proposition 2.4.18. An irreducible character $\chi \in \operatorname{Irr}(W)$ is a block of $\mathcal{O}_{\mathfrak{p O}} \mathcal{H}_{\phi}$ by itself if and only if $s_{\chi_{\phi}} \notin \mathfrak{p} \mathbb{Z}_{K}\left[y, y^{-1}\right]$.

Proposition 3.2.5. Let $C$ be a block of $A_{\mathfrak{p} A} \mathcal{H}$. If $M$ is an essential monomial for $W$ which is not $\mathfrak{p}$-essential for any $\chi \in C$, then $C$ is a block of $A_{\mathfrak{q}_{M}} \mathcal{H}$, where $\mathfrak{q}_{M}=(M-1) A+\mathfrak{p} A$.

Proposition 4.3.8. If $\chi, \psi \in \operatorname{Irr}(W)$ belong to the same block of $\mathcal{O}_{\mathfrak{p O}} \mathcal{H}_{\phi}$, then they are in the same $p$-block of $W$.

Proposition 4.4.6. If $\chi, \psi \in \operatorname{Irr}(W)$ are in the same block of $\mathcal{O}_{\mathfrak{p O}} \mathcal{H}_{\phi}$, then they are in the same Rouquier block of $\mathcal{H}_{\phi}$ and we have

$$
a_{\chi_{\phi}}+A_{\chi_{\phi}}=a_{\psi_{\phi}}+A_{\psi_{\phi}}
$$

\subsection{The exceptional irreducible complex re- flection groups}

Let $W:=G_{n}(4 \leq n \leq 37)$ be an irreducible exceptional complex reflection group with field of definition $K$.

If $n \in\{23,24,27,29,30,31,33,34,35,36,37\}$, then $W$ has only one hyperplane orbit $\mathcal{C}$ with $e_{\mathcal{C}}=2$. The generic Hecke algebra of $W$ is defined over a Laurent polynomial ring in two indeterminates $v_{\mathcal{C}, 0}$ and $v_{\mathcal{C}, 1}$ and the only essential monomial for $W$ is $v_{\mathcal{C}, 0} v_{\mathcal{C}, 1}^{-1}$.

If $\phi$ is the "spetsial" cyclotomic specialization (see Example 4.3.2), then $\phi$ is associated with no essential hyperplane for $W$. The $\mathfrak{p}$-blocks, for all $\phi$ bad prime ideals $\mathfrak{p}$, and the Rouquier blocks of the spetsial cyclotomic Hecke algebra of these groups have been calculated by Malle and Rouquier in [52].

If $\phi$ is a cyclotomic specialization associated with the unique essential hyperplane for $W$, then $\mathcal{H}_{\phi}$ is isomorphic to the group algebra $\mathbb{Z}_{K} W$. Its $p$ blocks are known from Brauer theory, whereas there exists a single Rouquier block (see also [57], §3, Rem.1).

Therefore, we will only study in detail the remaining cases. 


\subsubsection{Essential hyperplanes}

Let $\mathfrak{p}_{1}, \mathfrak{p}_{2}$ be two prime ideal of $\mathbb{Z}_{K}$ lying over the same prime number $p$. If $\Psi$ is a $K$-cyclotomic polynomial, then $\Psi(1) \in \mathfrak{p}_{1}$ if and only if $\Psi_{1} \in \mathfrak{p}_{2}$. We deduce that an essential hyperplane is $\mathfrak{p}_{1}$-essential for $W$ if and only if it is $\mathfrak{p}_{2}$-essential for $W$. Therefore, we can talk about determining the $p$-essential hyperplanes for $W$, where $p$ runs over the set of prime divisors of $|W|$.

Together with Jean Michel, we have programmed into the GAP package CHEVIE the Schur elements of the generic Hecke algebras of all exceptional irreducible complex reflection groups in factorized form (function SchurModels and SchurData). Given a prime ideal $\mathfrak{p}$ of $\mathbb{Z}_{K}$, GAP provides us with a way to determine whether an element of $\mathbb{Z}_{K}$ belongs to $\mathfrak{p}$. Therefore, we can easily determine the $p$-essential monomials and thus, the $p$-essential hyperplanes for $W$.

In particular, we only need to follow this procedure for the groups $G_{7}$, $G_{11}, G_{19}, G_{26}, G_{28}$ and $G_{32}$. In the Appendix, we give the specializations of the parameters which make

- $\mathcal{H}\left(G_{7}\right)$ the twisted symmetric algebra of some finite cyclic group over $\mathcal{H}\left(G_{4}\right), \mathcal{H}\left(G_{5}\right)$ and $\mathcal{H}\left(G_{6}\right)$.

- $\mathcal{H}\left(G_{11}\right)$ the twisted symmetric algebra of some finite cyclic group over $\mathcal{H}\left(G_{8}\right), \mathcal{H}\left(G_{9}\right), \mathcal{H}\left(G_{10}\right), \mathcal{H}\left(G_{12}\right), \mathcal{H}\left(G_{13}\right), \mathcal{H}\left(G_{14}\right)$ and $\mathcal{H}\left(G_{15}\right)$.

- $\mathcal{H}\left(G_{19}\right)$ the twisted symmetric algebra of some finite cyclic group over $\mathcal{H}\left(G_{16}\right), \mathcal{H}\left(G_{17}\right), \mathcal{H}\left(G_{18}\right), \mathcal{H}\left(G_{20}\right), \mathcal{H}\left(G_{21}\right)$ and $\mathcal{H}\left(G_{22}\right)$.

- $\mathcal{H}\left(G_{26}\right)$ the twisted symmetric algebra of the cyclic group $C_{2}$ over $\mathcal{H}\left(G_{25}\right)$.

In all these cases, Proposition 2.3.15 implies that the Schur elements of the twisted symmetric algebra are scalar multiples of the Schur elements of the subalgebra. Due to the nature of the specializations, we can obtain the essential hyperplanes for the smaller group from the ones of the larger.

Example 5.2.1 The essential hyperplanes for $G_{7}$ are given in Example 5.2.3 (note that different letters represent different hyperplane orbits). The only 3essential hyperplanes for $G_{7}$ are:

$$
\begin{aligned}
& c_{1}-c_{2}=0, \quad c_{0}-c_{1}=0, \quad c_{0}-c_{2}=0 \\
& b_{1}-b_{2}=0, \quad b_{0}-b_{1}=0, \quad b_{0}-b_{2}=0
\end{aligned}
$$

All its remaining essential hyperplanes are strictly 2 -essential. From these, we can obtain the $p$-essential hyperplanes (where $p=2,3$ ) 
- for $G_{6}$ by setting $b_{0}=b_{1}=b_{2}=0$,

- for $G_{5}$ by setting $a_{0}=a_{1}=0$,

- for $G_{4}$ by setting $a_{0}=a_{1}=b_{0}=b_{1}=b_{2}=0$.

We have created the GAP function EssentialHyperplanes which is applied as follows:

gap> EssentialHyperplanes (W, p) ;

and returns

- the essential hyperplanes for $W$, if $p=0$.

- the $p$-essential hyperplanes for $W$, if $p$ divides the order of $W$.

- error, if $p$ does not divide the order of $W$.

\section{Example 5.2.2}

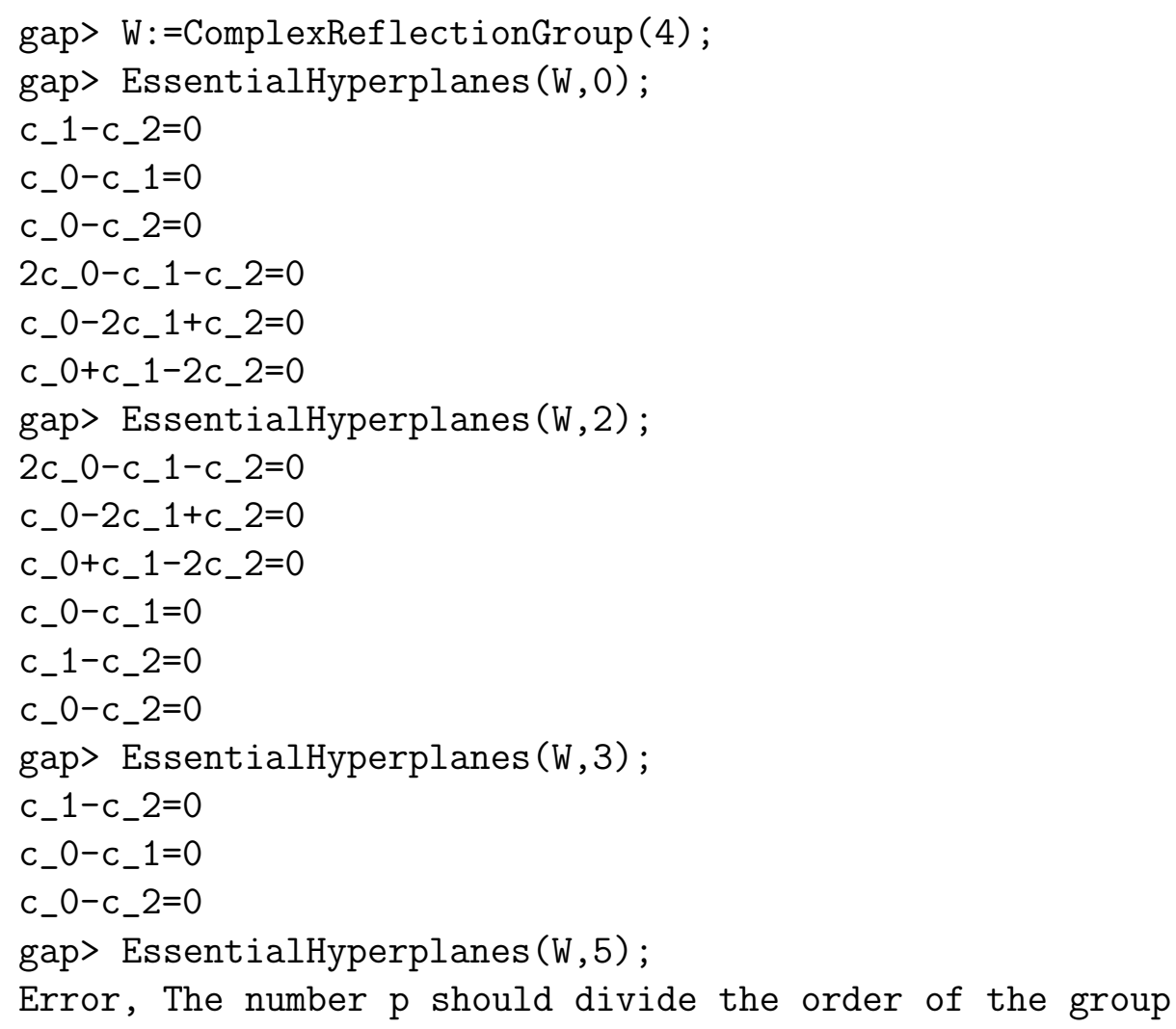




\subsubsection{Algorithm}

Let $\mathfrak{p}$ be a prime ideal of $\mathbb{Z}_{K}$ lying over a prime number $p$ which divides the order of the group $W$. In this section, we will present an algorithm for the determination of the $\mathfrak{p}$-blocks associated with no and each essential hyperplane for $W$. We retake here the notations of section 5.1 .

If we are interested in calculating the blocks of $A_{\mathfrak{p} A} \mathcal{H}$, we follow the steps below:

1. We select the characters $\chi \in \operatorname{Irr}(W)$ whose generic Schur elements belong to $\mathfrak{p} A$. The remaining ones will be blocks of $A_{\mathfrak{p} A} \mathcal{H}$ by themselves, due to Proposition 2.4.18. Thus we form a first partition $\lambda_{1}$ of $\operatorname{Irr}(W)$; one part formed by the selected characters, each remaining character forming a part by itself.

2. We calculate the $p$-blocks of $W$. By Proposition 4.3.8, if two irreducible characters are not in the same $p$-block of $W$, then they can not be in the same block of $A_{\mathfrak{p} A} \mathcal{H}$. We intersect the partition $\lambda_{1}$ with the partition obtained by the $p$-blocks of $W$ and we obtain a finer partition, named $\lambda_{2}$.

3. We find a cyclotomic specialization $\phi: v_{\mathcal{C}, j} \mapsto y^{n_{\mathcal{C}, j}}$ associated with no essential hyperplane by trying and checking random values for the $n_{\mathcal{C}, j}$. Following Proposition 4.4.6, we take the intersection of the partition we already have with the subsets of $\operatorname{Irr}(W)$, where the sum $a_{\chi_{\phi}}+A_{\chi_{\phi}}$ remains constant. This procedure is repeated several times, because sometimes the partition becomes finer after some repetitions. Finally, we obtain the partition $\lambda_{3}$, which is the finest of all.

If we are interested in calculating the blocks of $A_{\mathfrak{q}_{M}} \mathcal{H}$ for some $\mathfrak{p}$-essential monomial $M$, the procedure is more or less the same:

1. We select the characters $\chi \in \operatorname{Irr}(W)$ for which $M$ is a p-essential monomial. We form a first partition $\lambda_{1}$ of $\operatorname{Irr}(W)$; one part formed by the selected characters, each remaining character forming a part by itself. The idea is that, by Proposition 3.2.5, if $M$ is not $\mathfrak{p}$-essential for any character in a block $C$ of $A_{\mathfrak{p} A} \mathcal{H}$, then $C$ is a block of $A_{\mathfrak{q}_{M}} \mathcal{H}$. This explains step 4.

2. We calculate the $p$-blocks of $W$. By Proposition 4.3.8, if two irreducible characters are not in the same $p$-block of $W$, then they can not be in the same block of $A_{\mathfrak{q}_{M}} \mathcal{H}$. We intersect the partition $\lambda_{1}$ with the partition 
obtained by the $p$-blocks of $W$ and we obtain a finer partition, named $\lambda_{2}$.

3. We find a cyclotomic specialization $\phi: v_{\mathcal{C}, j} \mapsto y^{n_{\mathcal{C}, j}}$ associated with the $\mathfrak{p}$-essential hyperplane defined by $M$ (again by trying and checking random values for the $n_{\mathcal{C}, j}$ ). We repeat the third step as described for $A_{\mathfrak{p} A} \mathcal{H}$ to obtain partition $\lambda_{3}$.

4. We take the union of $\lambda_{3}$ and the partition defined by the blocks of $A_{\mathfrak{p} A} \mathcal{H}$.

The above algorithm is, due to step 3, heuristic. However, we will see in the next section that we only need to apply this algorithm to the groups $G_{7}$, $G_{11}, G_{19}, G_{26}, G_{28}$ and $G_{32}$. In these cases, we have been able to determine (using again the criteria presented in section 5.1) that the partition obtained at the end is minimal and corresponds to the blocks we are looking for.

Remark: Eventually, the above algorithm provides us with the correct Rouquier blocks for all exceptional irreducible complex reflection groups, except for $G_{34}$.

Remark: If $\mathfrak{p}_{1}, \mathfrak{p}_{2}$ are two prime ideals of $\mathbb{Z}_{K}$ lying over the same prime number $p$, we have observed that, for all exceptional irreducible complex reflection groups, the $\mathfrak{p}_{1}$-blocks always coincide with the $\mathfrak{p}_{2}$-blocks. Therefore, we can talk about determining the $p$-blocks associated with no and each essential hyperplane.

\subsubsection{Results}

With the help of the GAP package CHEVIE, we created a program which implements the algorithm of the previous section. Using Proposition 4.4.4, we have been able to determine the Rouquier blocks associated with no and each essential hyperplane for the groups $G_{7}, G_{11}, G_{19}, G_{26}, G_{28}$ and $G_{32}$.

Now, Clifford theory allows us to calculate the Rouquier blocks associated with no and each essential hyperplane for the remaining exceptional irreducible complex reflection groups. In all the cases presented in the Appendix, the explicit calculation of the blocks of the twisted symmetric algebras with the use of the algorithm of the previous section has shown that the assumptions of Corollary 2.3.19 are satisfied. Moreover, in all these cases, if $H$ is the twisted symmetric algebra of the finite cyclic group $G$ over $\bar{H}$, then each irreducible character of $H$ restricts to an irreducible character of $\bar{H}$. Using the notations of Proposition 2.3.15, this means that $|\bar{\Omega}|=1$, whence 
the blocks of $\bar{H}$ are stable under the action of $G$. We deduce that the blockidempotents of $H$ and $\bar{H}$ over the Rouquier ring coincide. In particular, if $C$ is a block (of characters) of $H$, then $\left\{\operatorname{Res}_{\bar{H}}^{H}(\chi) \mid \chi \in C\right\}$ is a block of $\bar{H}$.

We will give here the example of $G_{7}$ and show how we obtain the blocks of $G_{6}$ from those of $G_{7}$. Nevertheless, let us first explain the notations of characters used by the CHEVIE package.

Let $W$ be an exceptional irreducible complex reflection group. For $\chi \in$ $\operatorname{Irr}(W)$, we set $d(\chi):=\chi(1)$ and we denote by $b(\chi)$ the valuation of the fake degree of $\chi$ (for the definition of the fake degree see [16], 1.20). The irreducible characters $\chi$ of $W$ are determined by the corresponding pairs $(d(\chi), b(\chi))$ and we write $\chi=\phi_{d, b}$, where $d:=d(\chi)$ and $b:=b(\chi)$. If two irreducible characters $\chi$ and $\chi^{\prime}$ have $d(\chi)=d\left(\chi^{\prime}\right)$ and $b(\chi)=b\left(\chi^{\prime}\right)$, we use primes " " to distinguish them (following [51], [52]).

Example 5.2.3 The generic Hecke algebra of $G_{7}$ is

$$
\begin{aligned}
\mathcal{H}\left(G_{7}\right)=<S, T, U \mid & S T U=T U S=U S T \\
& \left(S-x_{0}\right)\left(S-x_{1}\right)=0 \\
& \left(T-y_{0}\right)\left(T-y_{1}\right)\left(T-y_{2}\right)=0 \\
& \left(U-z_{0}\right)\left(U-z_{1}\right)\left(U-z_{2}\right)=0>
\end{aligned}
$$

Let

$$
\phi: \begin{cases}x_{i} \mapsto\left(\zeta_{2}\right)^{i} q^{a_{i}} & (0 \leq i<2), \\ y_{j} \mapsto\left(\zeta_{3}\right)^{j} q^{b_{j}} & (0 \leq j<3), \\ z_{k} \mapsto\left(\zeta_{3}\right)^{k} q^{c_{k}} & (0 \leq k<3)\end{cases}
$$

be a cyclotomic specialization of $\mathcal{H}\left(G_{7}\right)$. The only prime numbers which divide the order of $G_{7}$ are 2 and 3. Using the algorithm of the previous section, we have determined the Rouquier blocks associated with no and each essential hyperplane for $G_{7}$. We present here only the non-trivial ones:

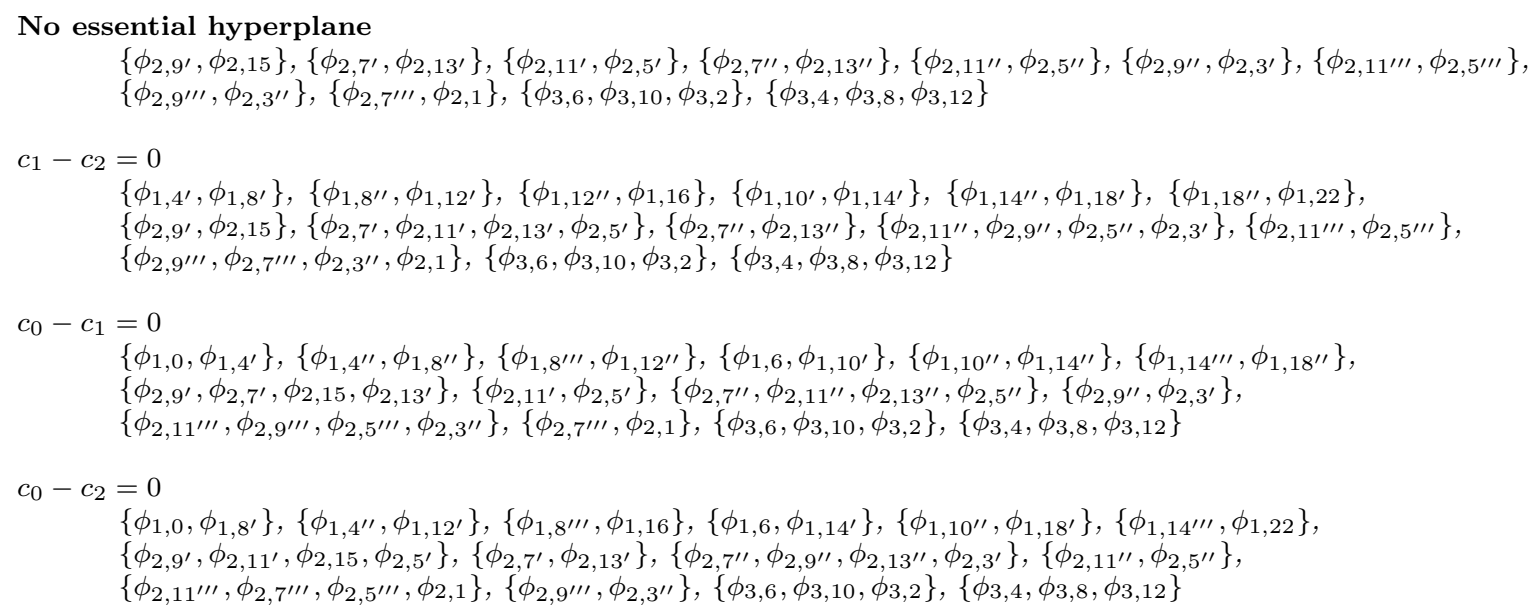


$b_{1}-b_{2}=0$

$\left\{\phi_{1,4^{\prime \prime}}, \phi_{1,8^{\prime \prime \prime}}\right\},\left\{\phi_{1,8^{\prime \prime}}, \phi_{1,12^{\prime \prime}}\right\},\left\{\phi_{1,12^{\prime}}, \phi_{1,16}\right\},\left\{\phi_{1,10^{\prime \prime}}, \phi_{1,14^{\prime \prime \prime}}\right\},\left\{\phi_{1,14^{\prime \prime}}, \phi_{1,18^{\prime \prime}}\right\}$,

$\left\{\phi_{1,18^{\prime}}, \phi_{1,22}\right\},\left\{\phi_{2,9^{\prime}}, \phi_{2,15}\right\},\left\{\phi_{2,7^{\prime}}, \phi_{2,13^{\prime}}\right\},\left\{\phi_{2,11^{\prime}}, \phi_{2,5^{\prime}}\right\},\left\{\phi_{2,7^{\prime \prime}}, \phi_{2,11^{\prime \prime \prime}}, \phi_{2,13^{\prime \prime}}, \phi_{2,5^{\prime \prime \prime}}\right\}$,

$\left\{\phi_{2,11^{\prime \prime}}, \phi_{2,9^{\prime \prime \prime}}, \phi_{2,5^{\prime \prime}}, \phi_{2,3^{\prime \prime}}\right\},\left\{\phi_{2,9^{\prime \prime}}, \phi_{2,7^{\prime \prime \prime}}, \phi_{2,3^{\prime}}, \phi_{2,1}\right\},\left\{\phi_{3,6}, \phi_{3,10}, \phi_{3,2}\right\},\left\{\phi_{3,4}, \phi_{3,8}, \phi_{3,12}\right\}$

$b_{0}-b_{1}=0$

$\left\{\phi_{1,0}, \phi_{1,4^{\prime \prime}}\right\},\left\{\phi_{1,4^{\prime}}, \phi_{1,8^{\prime \prime}}\right\},\left\{\phi_{1,8^{\prime}}, \phi_{1,12^{\prime}}\right\},\left\{\phi_{1,6}, \phi_{1,10^{\prime \prime}}\right\},\left\{\phi_{1,10^{\prime}}, \phi_{1,14^{\prime \prime}}\right\},\left\{\phi_{1,14^{\prime}}, \phi_{1,18^{\prime}}\right\}$,

$\left\{\phi_{2,9^{\prime}}, \phi_{2,7^{\prime \prime}}, \phi_{2,15}, \phi_{2,13^{\prime \prime}}\right\},\left\{\phi_{2,7^{\prime}}, \phi_{2,11^{\prime \prime}}, \phi_{2,13^{\prime}}, \phi_{2,5^{\prime \prime}}\right\},\left\{\phi_{2,11^{\prime}}, \phi_{2,9^{\prime \prime}}, \phi_{2,5^{\prime}}, \phi_{2,3^{\prime}}\right\}$,

$\left\{\phi_{2,11^{\prime \prime \prime}}, \phi_{2,5^{\prime \prime \prime}}\right\},\left\{\phi_{2,9^{\prime \prime \prime}}, \phi_{2,3^{\prime \prime}}\right\},\left\{\phi_{2,7^{\prime \prime \prime}}, \phi_{2,1}\right\},\left\{\phi_{3,6}, \phi_{3,10}, \phi_{3,2}\right\},\left\{\phi_{3,4}, \phi_{3,8}, \phi_{3,12}\right\}$

$b_{0}-b_{2}=0$

$\left\{\phi_{1,0}, \phi_{1,8^{\prime \prime \prime}}\right\},\left\{\phi_{1,4^{\prime}}, \phi_{1,12^{\prime \prime}}\right\},\left\{\phi_{1,8^{\prime}}, \phi_{1,16}\right\},\left\{\phi_{1,6}, \phi_{1,14^{\prime \prime \prime}}\right\},\left\{\phi_{1,10^{\prime}}, \phi_{1,18^{\prime \prime}}\right\},\left\{\phi_{1,14^{\prime}}, \phi_{1,22}\right\}$,

$\left\{\phi_{2,9^{\prime}}, \phi_{2,11^{\prime \prime \prime}}, \phi_{2,15}, \phi_{2,5^{\prime \prime \prime}}\right\},\left\{\phi_{2,7^{\prime}}, \phi_{2,9^{\prime \prime \prime}}, \phi_{2,13^{\prime}}, \phi_{2,3^{\prime \prime}}\right\},\left\{\phi_{2,11^{\prime}}, \phi_{2,7^{\prime \prime \prime}}, \phi_{2,5^{\prime}}, \phi_{2,1}\right\},\left\{\phi_{2,7^{\prime \prime}}, \phi_{2,13^{\prime \prime}}\right\}$, $\left\{\phi_{2,11^{\prime \prime}}, \phi_{2,5^{\prime \prime}}\right\},\left\{\phi_{2,9^{\prime \prime}}, \phi_{2,3^{\prime}}\right\},\left\{\phi_{3,6}, \phi_{3,10}, \phi_{3,2}\right\},\left\{\phi_{3,4}, \phi_{3,8}, \phi_{3,12}\right\}$

$a_{0}-a_{1}-2 b_{0}+b_{1}+b_{2}-2 c_{0}+c_{1}+c_{2}=0$

$\left\{\phi_{1,6}, \phi_{2,9^{\prime}}, \phi_{2,15}, \phi_{3,4}, \phi_{3,8}, \phi_{3,12}\right\},\left\{\phi_{2,7^{\prime}}, \phi_{2,13^{\prime}}\right\},\left\{\phi_{2,11^{\prime}}, \phi_{2,5^{\prime}}\right\},\left\{\phi_{2,7^{\prime \prime}}, \phi_{2,13^{\prime \prime}}\right\},\left\{\phi_{2,11^{\prime \prime}}, \phi_{2,5^{\prime \prime}}\right\}$,

$\left\{\phi_{2,9^{\prime \prime}}, \phi_{2,3^{\prime}}\right\},\left\{\phi_{2,11^{\prime \prime \prime}}, \phi_{2,5^{\prime \prime \prime}}\right\},\left\{\phi_{2,9^{\prime \prime \prime}}, \phi_{2,3^{\prime \prime}}\right\},\left\{\phi_{2,7^{\prime \prime \prime}}, \phi_{2,1}\right\},\left\{\phi_{3,6}, \phi_{3,10}, \phi_{3,2}\right\}$

$a_{0}-a_{1}-2 b_{0}+b_{1}+b_{2}+c_{0}-2 c_{1}+c_{2}=0$

$\left\{\phi_{1,10^{\prime}}, \phi_{2,7^{\prime}}, \phi_{2,13^{\prime}}, \phi_{3,4}, \phi_{3,8}, \phi_{3,12}\right\},\left\{\phi_{2,9^{\prime}}, \phi_{2,15}\right\},\left\{\phi_{2,11^{\prime}}, \phi_{2,5^{\prime}}\right\},\left\{\phi_{2,7^{\prime \prime}}, \phi_{2,13^{\prime \prime}}\right\},\left\{\phi_{2,11^{\prime \prime}}, \phi_{2,5^{\prime \prime}}\right\}$, $\left\{\phi_{2,9^{\prime \prime}}, \phi_{2,3^{\prime}}\right\},\left\{\phi_{2,11^{\prime \prime \prime}}, \phi_{2,5^{\prime \prime \prime}}\right\},\left\{\phi_{2,9^{\prime \prime \prime}}, \phi_{2,3^{\prime \prime}}\right\},\left\{\phi_{2,7^{\prime \prime \prime}}, \phi_{2,1}\right\},\left\{\phi_{3,6}, \phi_{3,10}, \phi_{3,2}\right\}$

$a_{0}-a_{1}-2 b_{0}+b_{1}+b_{2}+c_{0}+c_{1}-2 c_{2}=0$

$\left\{\phi_{1,14^{\prime}}, \phi_{2,11^{\prime}}, \phi_{2,5^{\prime}}, \phi_{3,4}, \phi_{3,8}, \phi_{3,12}\right\},\left\{\phi_{2,9^{\prime}}, \phi_{2,15}\right\},\left\{\phi_{2,7^{\prime}}, \phi_{2,13^{\prime}}\right\},\left\{\phi_{2,7^{\prime \prime}}, \phi_{2,13^{\prime \prime}}\right\},\left\{\phi_{2,11^{\prime \prime}}, \phi_{2,5^{\prime \prime}}\right\}$, $\left\{\phi_{2,9^{\prime \prime}}, \phi_{2,3^{\prime}}\right\},\left\{\phi_{2,11^{\prime \prime \prime}}, \phi_{2,5^{\prime \prime \prime}}\right\},\left\{\phi_{2,9^{\prime \prime \prime}}, \phi_{2,3^{\prime \prime}}\right\},\left\{\phi_{2,7^{\prime \prime \prime}}, \phi_{2,1}\right\},\left\{\phi_{3,6}, \phi_{3,10}, \phi_{3,2}\right\}$

$a_{0}-a_{1}-b_{0}-b_{1}+2 b_{2}-c_{0}-c_{1}+2 c_{2}=0$

$\left\{\phi_{1,16}, \phi_{2,7^{\prime \prime \prime}}, \phi_{2,1}, \phi_{3,6}, \phi_{3,10}, \phi_{3,2}\right\},\left\{\phi_{2,9^{\prime}}, \phi_{2,15}\right\},\left\{\phi_{2,7^{\prime}}, \phi_{2,13^{\prime}}\right\},\left\{\phi_{2,11^{\prime}}, \phi_{2,5^{\prime}}\right\},\left\{\phi_{2,7^{\prime \prime}}, \phi_{2,13^{\prime \prime}}\right\}$,

$\left\{\phi_{2,11^{\prime \prime}}, \phi_{2,5^{\prime \prime}}\right\},\left\{\phi_{2,9^{\prime \prime}}, \phi_{2,3^{\prime}}\right\},\left\{\phi_{2,11^{\prime \prime \prime}}, \phi_{2,5^{\prime \prime \prime}}\right\},\left\{\phi_{2,9^{\prime \prime \prime}}, \phi_{2,3^{\prime \prime}}\right\},\left\{\phi_{3,4}, \phi_{3,8}, \phi_{3,12}\right\}$

$a_{0}-a_{1}-b_{0}-b_{1}+2 b_{2}-c_{0}+2 c_{1}-c_{2}=0$

$\left\{\phi_{1,12^{\prime \prime}}, \phi_{2,9^{\prime \prime \prime}}, \phi_{2,3^{\prime \prime}}, \phi_{3,6}, \phi_{3,10}, \phi_{3,2}\right\},\left\{\phi_{2,9^{\prime}}, \phi_{2,15}\right\},\left\{\phi_{2,7^{\prime}}, \phi_{2,13^{\prime}}\right\},\left\{\phi_{2,11^{\prime}}, \phi_{2,5^{\prime}}\right\},\left\{\phi_{2,7^{\prime \prime}}, \phi_{2,13^{\prime \prime}}\right\}$,

$\left\{\phi_{2,11^{\prime \prime}}, \phi_{2,5^{\prime \prime}}\right\},\left\{\phi_{2,9^{\prime \prime}}, \phi_{2,3^{\prime}}\right\},\left\{\phi_{2,11^{\prime \prime \prime}}, \phi_{2,5^{\prime \prime \prime}}\right\},\left\{\phi_{2,7^{\prime \prime \prime}}, \phi_{2,1}\right\},\left\{\phi_{3,4}, \phi_{3,8}, \phi_{3,12}\right\}$

$a_{0}-a_{1}-b_{0}-b_{1}+2 b_{2}+2 c_{0}-c_{1}-c_{2}=0$

$\left\{\phi_{1,8^{\prime \prime \prime}}, \phi_{2,11^{\prime \prime \prime}}, \phi_{2,5^{\prime \prime \prime}}, \phi_{3,6}, \phi_{3,10}, \phi_{3,2}\right\},\left\{\phi_{2,9^{\prime}}, \phi_{2,15}\right\},\left\{\phi_{2,7^{\prime}}, \phi_{2,13^{\prime}}\right\},\left\{\phi_{2,11^{\prime}}, \phi_{2,5^{\prime}}\right\},\left\{\phi_{2,7^{\prime \prime}}, \phi_{2,13^{\prime \prime}}\right\}$, $\left\{\phi_{2,11^{\prime \prime}}, \phi_{2,5^{\prime \prime}}\right\},\left\{\phi_{2,9^{\prime \prime}}, \phi_{2,3^{\prime}}\right\},\left\{\phi_{2,9^{\prime \prime \prime}}, \phi_{2,3^{\prime \prime}}\right\},\left\{\phi_{2,7^{\prime \prime \prime}}, \phi_{2,1}\right\},\left\{\phi_{3,4}, \phi_{3,8}, \phi_{3,12}\right\}$

$a_{0}-a_{1}-b_{0}+b_{2}-c_{0}+c_{1}=0$

$\left\{\phi_{1,12^{\prime \prime}}, \phi_{1,6}, \phi_{2,9^{\prime \prime}}, \phi_{2,3^{\prime}}\right\},\left\{\phi_{2,9^{\prime}}, \phi_{2,15}\right\},\left\{\phi_{2,7^{\prime}}, \phi_{2,13^{\prime}}\right\},\left\{\phi_{2,11^{\prime}}, \phi_{2,5^{\prime}}\right\},\left\{\phi_{2,7^{\prime \prime}}, \phi_{2,13^{\prime \prime}}\right\},\left\{\phi_{2,11^{\prime \prime}}, \phi_{2,5^{\prime \prime}}\right\}$, $\left\{\phi_{2,11^{\prime \prime \prime}}, \phi_{2,5^{\prime \prime \prime}}\right\},\left\{\phi_{2,9^{\prime \prime \prime}}, \phi_{2,3^{\prime \prime}}\right\},\left\{\phi_{2,7^{\prime \prime \prime}}, \phi_{2,1}\right\},\left\{\phi_{3,6}, \phi_{3,10}, \phi_{3,2}\right\},\left\{\phi_{3,4}, \phi_{3,8}, \phi_{3,12}\right\}$

$a_{0}-a_{1}-b_{0}+b_{2}-c_{1}+c_{2}=0$

$\left\{\phi_{1,16}, \phi_{1,10^{\prime}}, \phi_{2,7^{\prime \prime}}, \phi_{2,13^{\prime \prime}}\right\},\left\{\phi_{2,9^{\prime}}, \phi_{2,15}\right\},\left\{\phi_{2,7^{\prime}}, \phi_{2,13^{\prime}}\right\},\left\{\phi_{2,11^{\prime}}, \phi_{2,5^{\prime}}\right\},\left\{\phi_{2,11^{\prime \prime}}, \phi_{2,5^{\prime \prime}}\right\},\left\{\phi_{2,9^{\prime \prime}}, \phi_{2,3^{\prime}}\right\}$, $\left\{\phi_{2,11^{\prime \prime \prime}}, \phi_{2,5^{\prime \prime \prime}}\right\},\left\{\phi_{2,9^{\prime \prime \prime}}, \phi_{2,3^{\prime \prime}}\right\},\left\{\phi_{2,7^{\prime \prime \prime}}, \phi_{2,1}\right\},\left\{\phi_{3,6}, \phi_{3,10}, \phi_{3,2}\right\},\left\{\phi_{3,4}, \phi_{3,8}, \phi_{3,12}\right\}$

$a_{0}-a_{1}-b_{0}+b_{2}+c_{0}-c_{2}=0$

$\left\{\phi_{1,8^{\prime \prime \prime}}, \phi_{1,14^{\prime}}, \phi_{2,11^{\prime \prime}}, \phi_{2,5^{\prime \prime}}\right\},\left\{\phi_{2,9^{\prime}}, \phi_{2,15}\right\},\left\{\phi_{2,7^{\prime}}, \phi_{2,13^{\prime}}\right\},\left\{\phi_{2,11^{\prime}}, \phi_{2,5^{\prime}}\right\},\left\{\phi_{2,7^{\prime \prime}}, \phi_{2,13^{\prime \prime}}\right\},\left\{\phi_{2,9^{\prime \prime}}, \phi_{2,3^{\prime}}\right\}$, $\left\{\phi_{2,11^{\prime \prime \prime}}, \phi_{2,5^{\prime \prime \prime}}\right\},\left\{\phi_{2,9^{\prime \prime \prime}}, \phi_{2,3^{\prime \prime}}\right\},\left\{\phi_{2,7^{\prime \prime \prime}}, \phi_{2,1}\right\},\left\{\phi_{3,6}, \phi_{3,10}, \phi_{3,2}\right\},\left\{\phi_{3,4}, \phi_{3,8}, \phi_{3,12}\right\}$

$a_{0}-a_{1}-b_{0}+b_{1}-c_{0}+c_{2}=0$

$\left\{\phi_{1,12^{\prime}}, \phi_{1,6}, \phi_{2,9^{\prime \prime \prime}}, \phi_{2,3^{\prime \prime}}\right\},\left\{\phi_{2,9^{\prime}}, \phi_{2,15}\right\},\left\{\phi_{2,7^{\prime}}, \phi_{2,13^{\prime}}\right\},\left\{\phi_{2,11^{\prime}}, \phi_{2,5^{\prime}}\right\},\left\{\phi_{2,7^{\prime \prime}}, \phi_{2,13^{\prime \prime}}\right\},\left\{\phi_{2,11^{\prime \prime}}, \phi_{2,5^{\prime \prime}}\right\}$, $\left\{\phi_{2,9^{\prime \prime}}, \phi_{2,3^{\prime}}\right\},\left\{\phi_{2,11^{\prime \prime \prime}}, \phi_{2,5^{\prime \prime \prime}}\right\},\left\{\phi_{2,7^{\prime \prime \prime}}, \phi_{2,1}\right\},\left\{\phi_{3,6}, \phi_{3,10}, \phi_{3,2}\right\},\left\{\phi_{3,4}, \phi_{3,8}, \phi_{3,12}\right\}$

$a_{0}-a_{1}-b_{0}+b_{1}+c_{1}-c_{2}=0$

$\left\{\phi_{1,8^{\prime \prime}}, \phi_{1,14^{\prime}}, \phi_{2,11^{\prime \prime \prime}}, \phi_{2,5^{\prime \prime \prime}}\right\},\left\{\phi_{2,9^{\prime}}, \phi_{2,15}\right\},\left\{\phi_{2,7^{\prime}}, \phi_{2,13^{\prime}}\right\},\left\{\phi_{2,11^{\prime}}, \phi_{2,5^{\prime}}\right\},\left\{\phi_{2,7^{\prime \prime}}, \phi_{2,13^{\prime \prime}}\right\},\left\{\phi_{2,11^{\prime \prime}}, \phi_{2,5^{\prime \prime}}\right\}$, $\left\{\phi_{2,9^{\prime \prime}}, \phi_{2,3^{\prime}}\right\},\left\{\phi_{2,9^{\prime \prime \prime}}, \phi_{2,3^{\prime \prime}}\right\},\left\{\phi_{2,7^{\prime \prime \prime}}, \phi_{2,1}\right\},\left\{\phi_{3,6}, \phi_{3,10}, \phi_{3,2}\right\},\left\{\phi_{3,4}, \phi_{3,8}, \phi_{3,12}\right\}$

$a_{0}-a_{1}-b_{0}+b_{1}+c_{0}-c_{1}=0$

$\left\{\phi_{1,4^{\prime \prime}}, \phi_{1,10^{\prime}}, \phi_{2,7^{\prime \prime \prime}}, \phi_{2,1}\right\},\left\{\phi_{2,9^{\prime}}, \phi_{2,15}\right\},\left\{\phi_{2,7^{\prime}}, \phi_{2,13^{\prime}}\right\},\left\{\phi_{2,11^{\prime}}, \phi_{2,5^{\prime}}\right\},\left\{\phi_{2,7^{\prime \prime}}, \phi_{2,13^{\prime \prime}}\right\},\left\{\phi_{2,11^{\prime \prime}}, \phi_{2,5^{\prime \prime}}\right\}$, $\left\{\phi_{2,9^{\prime \prime}}, \phi_{2,3^{\prime}}\right\},\left\{\phi_{2,11^{\prime \prime \prime}}, \phi_{2,5^{\prime \prime \prime}}\right\},\left\{\phi_{2,9^{\prime \prime \prime}}, \phi_{2,3^{\prime \prime}}\right\},\left\{\phi_{3,6}, \phi_{3,10}, \phi_{3,2}\right\},\left\{\phi_{3,4}, \phi_{3,8}, \phi_{3,12}\right\}$ 
$a_{0}-a_{1}-b_{0}+2 b_{1}-b_{2}-c_{0}-c_{1}+2 c_{2}=0$

$\left\{\phi_{1,12^{\prime}}, \phi_{2,9^{\prime \prime}}, \phi_{2,3^{\prime}}, \phi_{3,6}, \phi_{3,10}, \phi_{3,2}\right\},\left\{\phi_{2,9^{\prime}}, \phi_{2,15}\right\},\left\{\phi_{2,7^{\prime}}, \phi_{2,13^{\prime}}\right\},\left\{\phi_{2,11^{\prime}}, \phi_{2,5^{\prime}}\right\},\left\{\phi_{2,7^{\prime \prime}}, \phi_{2,13^{\prime \prime}}\right\}$,

$\left\{\phi_{2,11^{\prime \prime}}, \phi_{2,5^{\prime \prime}}\right\},\left\{\phi_{2,11^{\prime \prime \prime}}, \phi_{2,5^{\prime \prime \prime}}\right\},\left\{\phi_{2,9^{\prime \prime \prime}}, \phi_{2,3^{\prime \prime}}\right\},\left\{\phi_{2,7^{\prime \prime \prime}}, \phi_{2,1}\right\},\left\{\phi_{3,4}, \phi_{3,8}, \phi_{3,12}\right\}$

$a_{0}-a_{1}-b_{0}+2 b_{1}-b_{2}-c_{0}+2 c_{1}-c_{2}=0$

$\left\{\phi_{1,8^{\prime \prime}}, \phi_{2,11^{\prime \prime}}, \phi_{2,5^{\prime \prime}}, \phi_{3,6}, \phi_{3,10}, \phi_{3,2}\right\},\left\{\phi_{2,9^{\prime}}, \phi_{2,15}\right\},\left\{\phi_{2,7^{\prime}}, \phi_{2,13^{\prime}}\right\},\left\{\phi_{2,11^{\prime}}, \phi_{2,5^{\prime}}\right\},\left\{\phi_{2,7^{\prime \prime}}, \phi_{2,13^{\prime \prime}}\right\}$,

$\left\{\phi_{2,9^{\prime \prime}}, \phi_{2,3^{\prime}}\right\},\left\{\phi_{2,11^{\prime \prime \prime}}, \phi_{2,5^{\prime \prime \prime}}\right\},\left\{\phi_{2,9^{\prime \prime \prime}}, \phi_{2,3^{\prime \prime}}\right\},\left\{\phi_{2,7^{\prime \prime \prime}}, \phi_{2,1}\right\},\left\{\phi_{3,4}, \phi_{3,8}, \phi_{3,12}\right\}$

$a_{0}-a_{1}-b_{0}+2 b_{1}-b_{2}+2 c_{0}-c_{1}-c_{2}=0$

$\left\{\phi_{1,4^{\prime \prime}}, \phi_{2,7^{\prime \prime}}, \phi_{2,13^{\prime \prime}}, \phi_{3,6}, \phi_{3,10}, \phi_{3,2}\right\},\left\{\phi_{2,9^{\prime}}, \phi_{2,15}\right\},\left\{\phi_{2,7^{\prime}}, \phi_{2,13^{\prime}}\right\},\left\{\phi_{2,11^{\prime}}, \phi_{2,5^{\prime}}\right\},\left\{\phi_{2,11^{\prime \prime}}, \phi_{2,5^{\prime \prime}}\right\}$,

$\left\{\phi_{2,9^{\prime \prime}}, \phi_{2,3^{\prime}}\right\},\left\{\phi_{2,11^{\prime \prime \prime}}, \phi_{2,5^{\prime \prime \prime}}\right\},\left\{\phi_{2,9^{\prime \prime \prime}}, \phi_{2,3^{\prime \prime}}\right\},\left\{\phi_{2,7^{\prime \prime \prime}}, \phi_{2,1}\right\},\left\{\phi_{3,4}, \phi_{3,8}, \phi_{3,12}\right\}$

$a_{0}-a_{1}-b_{1}+b_{2}-c_{0}+c_{2}=0$

$\left\{\phi_{1,16}, \phi_{1,10^{\prime \prime}}, \phi_{2,7^{\prime}}, \phi_{2,13^{\prime}}\right\},\left\{\phi_{2,9^{\prime}}, \phi_{2,15}\right\},\left\{\phi_{2,11^{\prime}}, \phi_{2,5^{\prime}}\right\},\left\{\phi_{2,7^{\prime \prime}}, \phi_{2,13^{\prime \prime}}\right\},\left\{\phi_{2,11^{\prime \prime}}, \phi_{2,5^{\prime \prime}}\right\},\left\{\phi_{2,9^{\prime \prime}}, \phi_{2,3^{\prime}}\right\}$, $\left\{\phi_{2,11^{\prime \prime \prime}}, \phi_{2,5^{\prime \prime \prime}}\right\},\left\{\phi_{2,9^{\prime \prime \prime}}, \phi_{2,3^{\prime \prime}}\right\},\left\{\phi_{2,7^{\prime \prime \prime}}, \phi_{2,1}\right\},\left\{\phi_{3,6}, \phi_{3,10}, \phi_{3,2}\right\},\left\{\phi_{3,4}, \phi_{3,8}, \phi_{3,12}\right\}$

$a_{0}-a_{1}-b_{1}+b_{2}+c_{1}-c_{2}=0$

$\left\{\phi_{1,12^{\prime \prime}}, \phi_{1,18^{\prime}}, \phi_{2,9^{\prime}}, \phi_{2,15}\right\},\left\{\phi_{2,7^{\prime}}, \phi_{2,13^{\prime}}\right\},\left\{\phi_{2,11^{\prime}}, \phi_{2,5^{\prime}}\right\},\left\{\phi_{2,7^{\prime \prime}}, \phi_{2,13^{\prime \prime}}\right\},\left\{\phi_{2,11^{\prime \prime}}, \phi_{2,5^{\prime \prime}}\right\},\left\{\phi_{2,9^{\prime \prime}}, \phi_{2,3^{\prime}}\right\}$, $\left\{\phi_{2,11^{\prime \prime \prime}}, \phi_{2,5^{\prime \prime \prime}}\right\},\left\{\phi_{2,9^{\prime \prime \prime}}, \phi_{2,3^{\prime \prime}}\right\},\left\{\phi_{2,7^{\prime \prime \prime}}, \phi_{2,1}\right\},\left\{\phi_{3,6}, \phi_{3,10}, \phi_{3,2}\right\},\left\{\phi_{3,4}, \phi_{3,8}, \phi_{3,12}\right\}$

$a_{0}-a_{1}-b_{1}+b_{2}+c_{0}-c_{1}=0$

$\left\{\phi_{1,8^{\prime \prime \prime}}, \phi_{1,14^{\prime \prime}}, \phi_{2,11^{\prime}}, \phi_{2,5^{\prime}}\right\},\left\{\phi_{2,9^{\prime}}, \phi_{2,15}\right\},\left\{\phi_{2,7^{\prime}}, \phi_{2,13^{\prime}}\right\},\left\{\phi_{2,7^{\prime \prime}}, \phi_{2,13^{\prime \prime}}\right\},\left\{\phi_{2,11^{\prime \prime}}, \phi_{2,5^{\prime \prime}}\right\},\left\{\phi_{2,9^{\prime \prime}}, \phi_{2,3^{\prime}}\right\}$, $\left\{\phi_{2,11^{\prime \prime \prime}}, \phi_{2,5^{\prime \prime \prime}}\right\},\left\{\phi_{2,9^{\prime \prime \prime}}, \phi_{2,3^{\prime \prime}}\right\},\left\{\phi_{2,7^{\prime \prime \prime}}, \phi_{2,1}\right\},\left\{\phi_{3,6}, \phi_{3,10}, \phi_{3,2}\right\},\left\{\phi_{3,4}, \phi_{3,8}, \phi_{3,12}\right\}$

$a_{0}-a_{1}=0$

$\left\{\phi_{1,0}, \phi_{1,6}\right\},\left\{\phi_{1,4^{\prime}}, \phi_{1,10^{\prime}}\right\},\left\{\phi_{1,8^{\prime}}, \phi_{1,14^{\prime}}\right\},\left\{\phi_{1,4^{\prime \prime}}, \phi_{1,10^{\prime \prime}}\right\},\left\{\phi_{1,8^{\prime \prime}}, \phi_{1,14^{\prime \prime}}\right\},\left\{\phi_{1,12^{\prime}}, \phi_{1,18^{\prime}}\right\},\left\{\phi_{1,8^{\prime \prime \prime}}, \phi_{1,14^{\prime \prime \prime}}\right\}$, $\left\{\phi_{1,12^{\prime \prime}}, \phi_{1,18^{\prime \prime}}\right\},\left\{\phi_{1,16}, \phi_{1,22}\right\},\left\{\phi_{2,9^{\prime}}, \phi_{2,15}\right\},\left\{\phi_{2,7^{\prime}}, \phi_{2,13^{\prime}}\right\},\left\{\phi_{2,11^{\prime}}, \phi_{2,5^{\prime}}\right\},\left\{\phi_{2,7^{\prime \prime}}, \phi_{2,13^{\prime \prime}}\right\},\left\{\phi_{2,11^{\prime \prime}}, \phi_{2,5^{\prime \prime}}\right\}$, $\left\{\phi_{2,9^{\prime \prime}}, \phi_{2,3^{\prime}}\right\},\left\{\phi_{2,11^{\prime \prime \prime}}, \phi_{2,5^{\prime \prime \prime}}\right\},\left\{\phi_{2,9^{\prime \prime \prime}}, \phi_{2,3^{\prime \prime}}\right\},\left\{\phi_{2,7^{\prime \prime \prime}}, \phi_{2,1}\right\},\left\{\phi_{3,6}, \phi_{3,4}, \phi_{3,10}, \phi_{3,8}, \phi_{3,2}, \phi_{3,12}\right\}$

$a_{0}-a_{1}+b_{1}-b_{2}-c_{0}+c_{1}=0$

$\left\{\phi_{1,8^{\prime \prime}}, \phi_{1,14^{\prime \prime \prime}}, \phi_{2,11^{\prime}}, \phi_{2,5^{\prime}}\right\},\left\{\phi_{2,9^{\prime}}, \phi_{2,15}\right\},\left\{\phi_{2,7^{\prime}}, \phi_{2,13^{\prime}}\right\},\left\{\phi_{2,7^{\prime \prime}}, \phi_{2,13^{\prime \prime}}\right\},\left\{\phi_{2,11^{\prime \prime}}, \phi_{2,5^{\prime \prime}}\right\},\left\{\phi_{2,9^{\prime \prime}}, \phi_{2,3^{\prime}}\right\}$, $\left\{\phi_{2,11^{\prime \prime \prime}}, \phi_{2,5^{\prime \prime \prime}}\right\},\left\{\phi_{2,9^{\prime \prime \prime}}, \phi_{2,3^{\prime \prime}}\right\},\left\{\phi_{2,7^{\prime \prime \prime}}, \phi_{2,1}\right\},\left\{\phi_{3,6}, \phi_{3,10}, \phi_{3,2}\right\},\left\{\phi_{3,4}, \phi_{3,8}, \phi_{3,12}\right\}$

$a_{0}-a_{1}+b_{1}-b_{2}-c_{1}+c_{2}=0$

$\left\{\phi_{1,12^{\prime}}, \phi_{1,18^{\prime \prime}}, \phi_{2,9^{\prime}}, \phi_{2,15}\right\},\left\{\phi_{2,7^{\prime}}, \phi_{2,13^{\prime}}\right\},\left\{\phi_{2,11^{\prime}}, \phi_{2,5^{\prime}}\right\},\left\{\phi_{2,7^{\prime \prime}}, \phi_{2,13^{\prime \prime}}\right\},\left\{\phi_{2,11^{\prime \prime}}, \phi_{2,5^{\prime \prime}}\right\},\left\{\phi_{2,9^{\prime \prime}}, \phi_{2,3^{\prime}}\right\}$, $\left\{\phi_{2,11^{\prime \prime \prime}}, \phi_{2,5^{\prime \prime \prime}}\right\},\left\{\phi_{2,9^{\prime \prime \prime}}, \phi_{2,3^{\prime \prime}}\right\},\left\{\phi_{2,7^{\prime \prime \prime}}, \phi_{2,1}\right\},\left\{\phi_{3,6}, \phi_{3,10}, \phi_{3,2}\right\},\left\{\phi_{3,4}, \phi_{3,8}, \phi_{3,12}\right\}$

$a_{0}-a_{1}+b_{1}-b_{2}+c_{0}-c_{2}=0$

$\left\{\phi_{1,4^{\prime \prime}}, \phi_{1,22}, \phi_{2,7^{\prime}}, \phi_{2,13^{\prime}}\right\},\left\{\phi_{2,9^{\prime}}, \phi_{2,15}\right\},\left\{\phi_{2,11^{\prime}}, \phi_{2,5^{\prime}}\right\},\left\{\phi_{2,7^{\prime \prime}}, \phi_{2,13^{\prime \prime}}\right\},\left\{\phi_{2,11^{\prime \prime}}, \phi_{2,5^{\prime \prime}}\right\},\left\{\phi_{2,9^{\prime \prime}}, \phi_{2,3^{\prime}}\right\}$, $\left\{\phi_{2,11^{\prime \prime \prime}}, \phi_{2,5^{\prime \prime \prime}}\right\},\left\{\phi_{2,9^{\prime \prime \prime}}, \phi_{2,3^{\prime \prime}}\right\},\left\{\phi_{2,7^{\prime \prime \prime}}, \phi_{2,1}\right\},\left\{\phi_{3,6}, \phi_{3,10}, \phi_{3,2}\right\},\left\{\phi_{3,4}, \phi_{3,8}, \phi_{3,12}\right\}$

$a_{0}-a_{1}+b_{0}-2 b_{1}+b_{2}-2 c_{0}+c_{1}+c_{2}=0$

$\left\{\phi_{1,10^{\prime \prime}}, \phi_{2,7^{\prime \prime}}, \phi_{2,13^{\prime \prime}}, \phi_{3,4}, \phi_{3,8}, \phi_{3,12}\right\},\left\{\phi_{2,9^{\prime}}, \phi_{2,15}\right\},\left\{\phi_{2,7^{\prime}}, \phi_{2,13^{\prime}}\right\},\left\{\phi_{2,11^{\prime}}, \phi_{2,5^{\prime}}\right\},\left\{\phi_{2,11^{\prime \prime}}, \phi_{2,5^{\prime \prime}}\right\}$, $\left\{\phi_{2,9^{\prime \prime}}, \phi_{2,3^{\prime}}\right\},\left\{\phi_{2,11^{\prime \prime \prime}}, \phi_{2,5^{\prime \prime \prime}}\right\},\left\{\phi_{2,9^{\prime \prime \prime}}, \phi_{2,3^{\prime \prime}}\right\},\left\{\phi_{2,7^{\prime \prime \prime}}, \phi_{2,1}\right\},\left\{\phi_{3,6}, \phi_{3,10}, \phi_{3,2}\right\}$

$a_{0}-a_{1}+b_{0}-2 b_{1}+b_{2}+c_{0}-2 c_{1}+c_{2}=0$

$\left\{\phi_{1,14^{\prime \prime}}, \phi_{2,11^{\prime \prime}}, \phi_{2,5^{\prime \prime}}, \phi_{3,4}, \phi_{3,8}, \phi_{3,12}\right\},\left\{\phi_{2,9^{\prime}}, \phi_{2,15}\right\},\left\{\phi_{2,7^{\prime}}, \phi_{2,13^{\prime}}\right\},\left\{\phi_{2,11^{\prime}}, \phi_{2,5^{\prime}}\right\},\left\{\phi_{2,7^{\prime \prime}}, \phi_{2,13^{\prime \prime}}\right\}$,

$\left\{\phi_{2,9^{\prime \prime}}, \phi_{2,3^{\prime}}\right\},\left\{\phi_{2,11^{\prime \prime \prime}}, \phi_{2,5^{\prime \prime \prime}}\right\},\left\{\phi_{2,9^{\prime \prime \prime}}, \phi_{2,3^{\prime \prime}}\right\},\left\{\phi_{2,7^{\prime \prime \prime}}, \phi_{2,1}\right\},\left\{\phi_{3,6}, \phi_{3,10}, \phi_{3,2}\right\}$

$a_{0}-a_{1}+b_{0}-2 b_{1}+b_{2}+c_{0}+c_{1}-2 c_{2}=0$

$\left\{\phi_{1,18^{\prime}}, \phi_{2,9^{\prime \prime}}, \phi_{2,3^{\prime}}, \phi_{3,4}, \phi_{3,8}, \phi_{3,12}\right\},\left\{\phi_{2,9^{\prime}}, \phi_{2,15}\right\},\left\{\phi_{2,7^{\prime}}, \phi_{2,13^{\prime}}\right\},\left\{\phi_{2,11^{\prime}}, \phi_{2,5^{\prime}}\right\},\left\{\phi_{2,7^{\prime \prime}}, \phi_{2,13^{\prime \prime}}\right\}$,

$\left\{\phi_{2,11^{\prime \prime}}, \phi_{2,5^{\prime \prime}}\right\},\left\{\phi_{2,11^{\prime \prime \prime}}, \phi_{2,5^{\prime \prime \prime}}\right\},\left\{\phi_{2,9^{\prime \prime \prime}}, \phi_{2,3^{\prime \prime}}\right\},\left\{\phi_{2,7^{\prime \prime \prime}}, \phi_{2,1}\right\},\left\{\phi_{3,6}, \phi_{3,10}, \phi_{3,2}\right\}$

$a_{0}-a_{1}+b_{0}-b_{1}-c_{0}+c_{1}=0$

$\left\{\phi_{1,4^{\prime}}, \phi_{1,10^{\prime \prime}}, \phi_{2,7^{\prime \prime \prime}}, \phi_{2,1}\right\},\left\{\phi_{2,9^{\prime}}, \phi_{2,15}\right\},\left\{\phi_{2,7^{\prime}}, \phi_{2,13^{\prime}}\right\},\left\{\phi_{2,11^{\prime}}, \phi_{2,5^{\prime}}\right\},\left\{\phi_{2,7^{\prime \prime}}, \phi_{2,13^{\prime \prime}}\right\},\left\{\phi_{2,11^{\prime \prime}}, \phi_{2,5^{\prime \prime}}\right\}$, $\left\{\phi_{2,9^{\prime \prime}}, \phi_{2,3^{\prime}}\right\},\left\{\phi_{2,11^{\prime \prime \prime}}, \phi_{2,5^{\prime \prime \prime}}\right\},\left\{\phi_{2,9^{\prime \prime \prime}}, \phi_{2,3^{\prime \prime}}\right\},\left\{\phi_{3,6}, \phi_{3,10}, \phi_{3,2}\right\},\left\{\phi_{3,4}, \phi_{3,8}, \phi_{3,12}\right\}$

$a_{0}-a_{1}+b_{0}-b_{1}-c_{1}+c_{2}=0$

$\left\{\phi_{1,8^{\prime}}, \phi_{1,14^{\prime \prime}}, \phi_{2,11^{\prime \prime \prime}}, \phi_{2,5^{\prime \prime \prime}}\right\},\left\{\phi_{2,9^{\prime}}, \phi_{2,15}\right\},\left\{\phi_{2,7^{\prime}}, \phi_{2,13^{\prime}}\right\},\left\{\phi_{2,11^{\prime}}, \phi_{2,5^{\prime}}\right\},\left\{\phi_{2,7^{\prime \prime}}, \phi_{2,13^{\prime \prime}}\right\},\left\{\phi_{2,11^{\prime \prime}}, \phi_{2,5^{\prime \prime}}\right\}$, $\left\{\phi_{2,9^{\prime \prime}}, \phi_{2,3^{\prime}}\right\},\left\{\phi_{2,9^{\prime \prime \prime}}, \phi_{2,3^{\prime \prime}}\right\},\left\{\phi_{2,7^{\prime \prime \prime}}, \phi_{2,1}\right\},\left\{\phi_{3,6}, \phi_{3,10}, \phi_{3,2}\right\},\left\{\phi_{3,4}, \phi_{3,8}, \phi_{3,12}\right\}$ 
$a_{0}-a_{1}+b_{0}-b_{1}+c_{0}-c_{2}=0$

$\left\{\phi_{1,0}, \phi_{1,18^{\prime}}, \phi_{2,9^{\prime \prime \prime}}, \phi_{2,3^{\prime \prime}}\right\},\left\{\phi_{2,9^{\prime}}, \phi_{2,15}\right\},\left\{\phi_{2,7^{\prime}}, \phi_{2,13^{\prime}}\right\},\left\{\phi_{2,11^{\prime}}, \phi_{2,5^{\prime}}\right\},\left\{\phi_{2,7^{\prime \prime}}, \phi_{2,13^{\prime \prime}}\right\},\left\{\phi_{2,11^{\prime \prime}}, \phi_{2,5^{\prime \prime}}\right\}$, $\left\{\phi_{2,9^{\prime \prime}}, \phi_{2,3^{\prime}}\right\},\left\{\phi_{2,11^{\prime \prime \prime}}, \phi_{2,5^{\prime \prime \prime}}\right\},\left\{\phi_{2,7^{\prime \prime \prime}}, \phi_{2,1}\right\},\left\{\phi_{3,6}, \phi_{3,10}, \phi_{3,2}\right\},\left\{\phi_{3,4}, \phi_{3,8}, \phi_{3,12}\right\}$

$a_{0}-a_{1}+b_{0}-b_{2}-c_{0}+c_{2}=0$

$\left\{\phi_{1,8^{\prime}}, \phi_{1,14^{\prime \prime \prime}}, \phi_{2,11^{\prime \prime}}, \phi_{2,5^{\prime \prime}}\right\},\left\{\phi_{2,9^{\prime}}, \phi_{2,15}\right\},\left\{\phi_{2,7^{\prime}}, \phi_{2,13^{\prime}}\right\},\left\{\phi_{2,11^{\prime}}, \phi_{2,5^{\prime}}\right\},\left\{\phi_{2,7^{\prime \prime}}, \phi_{2,13^{\prime \prime}}\right\},\left\{\phi_{2,9^{\prime \prime}}, \phi_{2,3^{\prime}}\right\}$, $\left\{\phi_{2,11^{\prime \prime \prime}}, \phi_{2,5^{\prime \prime \prime}}\right\},\left\{\phi_{2,9^{\prime \prime \prime}}, \phi_{2,3^{\prime \prime}}\right\},\left\{\phi_{2,7^{\prime \prime \prime}}, \phi_{2,1}\right\},\left\{\phi_{3,6}, \phi_{3,10}, \phi_{3,2}\right\},\left\{\phi_{3,4}, \phi_{3,8}, \phi_{3,12}\right\}$

$a_{0}-a_{1}+b_{0}-b_{2}+c_{1}-c_{2}=0$

$\left\{\phi_{1,4^{\prime}}, \phi_{1,22}, \phi_{2,7^{\prime \prime}}, \phi_{2,13^{\prime \prime}}\right\},\left\{\phi_{2,9^{\prime}}, \phi_{2,15}\right\},\left\{\phi_{2,7^{\prime}}, \phi_{2,13^{\prime}}\right\},\left\{\phi_{2,11^{\prime}}, \phi_{2,5^{\prime}}\right\},\left\{\phi_{2,11^{\prime \prime}}, \phi_{2,5^{\prime \prime}}\right\},\left\{\phi_{2,9^{\prime \prime}}, \phi_{2,3^{\prime}}\right\}$, $\left\{\phi_{2,11^{\prime \prime \prime}}, \phi_{2,5^{\prime \prime \prime}}\right\},\left\{\phi_{2,9^{\prime \prime \prime}}, \phi_{2,3^{\prime \prime}}\right\},\left\{\phi_{2,7^{\prime \prime \prime}}, \phi_{2,1}\right\},\left\{\phi_{3,6}, \phi_{3,10}, \phi_{3,2}\right\},\left\{\phi_{3,4}, \phi_{3,8}, \phi_{3,12}\right\}$

$a_{0}-a_{1}+b_{0}-b_{2}+c_{0}-c_{1}=0$

$\left\{\phi_{1,0}, \phi_{1,18^{\prime \prime}}, \phi_{2,9^{\prime \prime}}, \phi_{2,3^{\prime}}\right\},\left\{\phi_{2,9^{\prime}}, \phi_{2,15}\right\},\left\{\phi_{2,7^{\prime}}, \phi_{2,13^{\prime}}\right\},\left\{\phi_{2,11^{\prime}}, \phi_{2,5^{\prime}}\right\},\left\{\phi_{2,7^{\prime \prime}}, \phi_{2,13^{\prime \prime}}\right\},\left\{\phi_{2,11^{\prime \prime}}, \phi_{2,5^{\prime \prime}}\right\}$, $\left\{\phi_{2,11^{\prime \prime \prime}}, \phi_{2,5^{\prime \prime \prime}}\right\},\left\{\phi_{2,9^{\prime \prime \prime}}, \phi_{2,3^{\prime \prime}}\right\},\left\{\phi_{2,7^{\prime \prime \prime}}, \phi_{2,1}\right\},\left\{\phi_{3,6}, \phi_{3,10}, \phi_{3,2}\right\},\left\{\phi_{3,4}, \phi_{3,8}, \phi_{3,12}\right\}$

$a_{0}-a_{1}+b_{0}+b_{1}-2 b_{2}-2 c_{0}+c_{1}+c_{2}=0$

$\left\{\phi_{1,14^{\prime \prime \prime}}, \phi_{2,11^{\prime \prime \prime}}, \phi_{2,5^{\prime \prime \prime}}, \phi_{3,4}, \phi_{3,8}, \phi_{3,12}\right\},\left\{\phi_{2,9^{\prime}}, \phi_{2,15}\right\},\left\{\phi_{2,7^{\prime}}, \phi_{2,13^{\prime}}\right\},\left\{\phi_{2,11^{\prime}}, \phi_{2,5^{\prime}}\right\},\left\{\phi_{2,7^{\prime \prime}}, \phi_{2,13^{\prime \prime}}\right\}$,

$\left\{\phi_{2,11^{\prime \prime}}, \phi_{2,5^{\prime \prime}}\right\},\left\{\phi_{2,9^{\prime \prime}}, \phi_{2,3^{\prime}}\right\},\left\{\phi_{2,9^{\prime \prime \prime}}, \phi_{2,3^{\prime \prime}}\right\},\left\{\phi_{2,7^{\prime \prime \prime}}, \phi_{2,1}\right\},\left\{\phi_{3,6}, \phi_{3,10}, \phi_{3,2}\right\}$

$a_{0}-a_{1}+b_{0}+b_{1}-2 b_{2}+c_{0}-2 c_{1}+c_{2}=0$

$\left\{\phi_{1,18^{\prime \prime}}, \phi_{2,9^{\prime \prime \prime}}, \phi_{2,3^{\prime \prime}}, \phi_{3,4}, \phi_{3,8}, \phi_{3,12}\right\},\left\{\phi_{2,9^{\prime}}, \phi_{2,15}\right\},\left\{\phi_{2,7^{\prime}}, \phi_{2,13^{\prime}}\right\},\left\{\phi_{2,11^{\prime}}, \phi_{2,5^{\prime}}\right\},\left\{\phi_{2,7^{\prime \prime}}, \phi_{2,13^{\prime \prime}}\right\}$,

$\left\{\phi_{2,11^{\prime \prime}}, \phi_{2,5^{\prime \prime}}\right\},\left\{\phi_{2,9^{\prime \prime}}, \phi_{2,3^{\prime}}\right\},\left\{\phi_{2,11^{\prime \prime \prime}}, \phi_{2,5^{\prime \prime \prime}}\right\},\left\{\phi_{2,7^{\prime \prime \prime}}, \phi_{2,1}\right\},\left\{\phi_{3,6}, \phi_{3,10}, \phi_{3,2}\right\}$

$a_{0}-a_{1}+b_{0}+b_{1}-2 b_{2}+c_{0}+c_{1}-2 c_{2}=0$

$\left\{\phi_{1,22}, \phi_{2,7^{\prime \prime \prime}}, \phi_{2,1}, \phi_{3,4}, \phi_{3,8}, \phi_{3,12}\right\},\left\{\phi_{2,9^{\prime}}, \phi_{2,15}\right\},\left\{\phi_{2,7^{\prime}}, \phi_{2,13^{\prime}}\right\},\left\{\phi_{2,11^{\prime}}, \phi_{2,5^{\prime}}\right\},\left\{\phi_{2,7^{\prime \prime}}, \phi_{2,13^{\prime \prime}}\right\}$,

$\left\{\phi_{2,11^{\prime \prime}}, \phi_{2,5^{\prime \prime}}\right\},\left\{\phi_{2,9^{\prime \prime}}, \phi_{2,3^{\prime}}\right\},\left\{\phi_{2,11^{\prime \prime \prime}}, \phi_{2,5^{\prime \prime \prime}}\right\},\left\{\phi_{2,9^{\prime \prime \prime}}, \phi_{2,3^{\prime \prime}}\right\},\left\{\phi_{3,6}, \phi_{3,10}, \phi_{3,2}\right\}$

$a_{0}-a_{1}+2 b_{0}-b_{1}-b_{2}-c_{0}-c_{1}+2 c_{2}=0$

$\left\{\phi_{1,8^{\prime}}, \phi_{2,11^{\prime}}, \phi_{2,5^{\prime}}, \phi_{3,6}, \phi_{3,10}, \phi_{3,2}\right\},\left\{\phi_{2,9^{\prime}}, \phi_{2,15}\right\},\left\{\phi_{2,7^{\prime}}, \phi_{2,13^{\prime}}\right\},\left\{\phi_{2,7^{\prime \prime}}, \phi_{2,13^{\prime \prime}}\right\},\left\{\phi_{2,11^{\prime \prime}}, \phi_{2,5^{\prime \prime}}\right\}$,

$\left\{\phi_{2,9^{\prime \prime}}, \phi_{2,3^{\prime}}\right\},\left\{\phi_{2,11^{\prime \prime \prime}}, \phi_{2,5^{\prime \prime \prime}}\right\},\left\{\phi_{2,9^{\prime \prime \prime}}, \phi_{2,3^{\prime \prime}}\right\},\left\{\phi_{2,7^{\prime \prime \prime}}, \phi_{2,1}\right\},\left\{\phi_{3,4}, \phi_{3,8}, \phi_{3,12}\right\}$

$a_{0}-a_{1}+2 b_{0}-b_{1}-b_{2}-c_{0}+2 c_{1}-c_{2}=0$

$\left\{\phi_{1,4^{\prime}}, \phi_{2,7^{\prime}}, \phi_{2,13^{\prime}}, \phi_{3,6}, \phi_{3,10}, \phi_{3,2}\right\},\left\{\phi_{2,9^{\prime}}, \phi_{2,15}\right\},\left\{\phi_{2,11^{\prime}}, \phi_{2,5^{\prime}}\right\},\left\{\phi_{2,7^{\prime \prime}}, \phi_{2,13^{\prime \prime}}\right\},\left\{\phi_{2,11^{\prime \prime}}, \phi_{2,5^{\prime \prime}}\right\}$,

$\left\{\phi_{2,9^{\prime \prime}}, \phi_{2,3^{\prime}}\right\},\left\{\phi_{2,11^{\prime \prime \prime}}, \phi_{2,5^{\prime \prime \prime}}\right\},\left\{\phi_{2,9^{\prime \prime \prime}}, \phi_{2,3^{\prime \prime}}\right\},\left\{\phi_{2,7^{\prime \prime \prime}}, \phi_{2,1}\right\},\left\{\phi_{3,4}, \phi_{3,8}, \phi_{3,12}\right\}$

$a_{0}-a_{1}+2 b_{0}-b_{1}-b_{2}+2 c_{0}-c_{1}-c_{2}=0$

$\left\{\phi_{1,0}, \phi_{2,9^{\prime}}, \phi_{2,15}, \phi_{3,6}, \phi_{3,10}, \phi_{3,2}\right\},\left\{\phi_{2,7^{\prime}}, \phi_{2,13^{\prime}}\right\},\left\{\phi_{2,11^{\prime}}, \phi_{2,5^{\prime}}\right\},\left\{\phi_{2,7^{\prime \prime}}, \phi_{2,13^{\prime \prime}}\right\},\left\{\phi_{2,11^{\prime \prime}}, \phi_{2,5^{\prime \prime}}\right\}$, $\left\{\phi_{2,9^{\prime \prime}}, \phi_{2,3^{\prime}}\right\},\left\{\phi_{2,11^{\prime \prime \prime}}, \phi_{2,5^{\prime \prime \prime}}\right\},\left\{\phi_{2,9^{\prime \prime \prime}}, \phi_{2,3^{\prime \prime}}\right\},\left\{\phi_{2,7^{\prime \prime \prime}}, \phi_{2,1}\right\},\left\{\phi_{3,4}, \phi_{3,8}, \phi_{3,12}\right\}$

Now, by Lemma 6.1.1, the generic Hecke algebra $\mathcal{H}\left(G_{6}\right)$ of $G_{6}$ is isomorphic to the subalgebra $\bar{H}:=<S, U>$ of the following specialization $H$ of $\mathcal{H}\left(G_{7}\right)$

$$
\begin{aligned}
H:=<S, T, U \mid & S T U=T U S=U S T, T^{3}=1 \\
& \left(S-x_{0}\right)\left(S-x_{1}\right)=0 \\
& \left(U-z_{0}\right)\left(U-z_{1}\right)\left(U-z_{2}\right)=0>
\end{aligned}
$$

The algebra $H$ is the twisted symmetric algebra of the cyclic group $C_{3}$ over the symmetric subalgebra $\bar{H}$ and this holds for all further specializations of the parameters. If we denote by $\phi$ the characters of $H$ and by $\psi$ the characters of $\bar{H}$, we 
have

$$
\begin{array}{lll}
\operatorname{Ind}_{\bar{H}}^{H}\left(\psi_{1,0}\right) & =\phi_{1,0}+\phi_{1,4^{\prime \prime}}+\phi_{1,8^{\prime \prime \prime}} & \operatorname{Ind}_{\bar{H}}^{H}\left(\psi_{1,4}\right)=\phi_{1,4^{\prime}}+\phi_{1,8^{\prime \prime}}+\phi_{1,12^{\prime \prime}} \\
\operatorname{Ind}_{\bar{H}}^{H}\left(\psi_{1,8}\right)=\phi_{1,8^{\prime}}+\phi_{1,12^{\prime}}+\phi_{1,16} & \operatorname{Ind}_{\bar{H}}^{H}\left(\psi_{1,6}\right)=\phi_{1,6}+\phi_{1,10^{\prime \prime}}+\phi_{1,14^{\prime \prime \prime}} \\
\operatorname{Ind}_{\bar{H}}^{H}\left(\psi_{1,10}\right)=\phi_{1,10^{\prime}}+\phi_{1,14^{\prime \prime}}+\phi_{1,18^{\prime \prime}} & \operatorname{Ind}_{\bar{H}}^{H}\left(\psi_{1,14}\right)=\phi_{1,14^{\prime}}+\phi_{1,18^{\prime}}+\phi_{1,22} \\
\operatorname{Ind}_{\bar{H}}^{H}\left(\psi_{2,5^{\prime \prime}}\right)=\phi_{2,9^{\prime}}+\phi_{2,13^{\prime \prime}}+\phi_{2,5^{\prime \prime \prime}} & \operatorname{Ind}_{\bar{H}}^{H}\left(\psi_{2,3^{\prime \prime}}\right)=\phi_{2,7^{\prime}}+\phi_{2,11^{\prime \prime}}+\phi_{2,3^{\prime \prime}} \\
\operatorname{Ind}_{\bar{H}}^{H}\left(\psi_{2,3^{\prime}}\right)=\phi_{2,11^{\prime}}+\phi_{2,7^{\prime \prime \prime}}+\phi_{2,3^{\prime}} & \operatorname{Ind}_{\bar{H}}^{H}\left(\psi_{2,7}\right)=\phi_{2,7^{\prime \prime}}+\phi_{2,11^{\prime \prime \prime}}+\phi_{2,15} \\
\operatorname{Ind}_{\bar{H}}^{H}\left(\psi_{2,1}\right)=\phi_{2,9^{\prime \prime}}+\phi_{2,5^{\prime}}+\phi_{2,1} & \operatorname{Ind}_{\bar{H}}^{H}\left(\psi_{2,5^{\prime}}\right)=\phi_{2,9^{\prime \prime \prime}}+\phi_{2,13^{\prime}}+\phi_{2,5^{\prime \prime}} \\
\operatorname{Ind}_{\bar{H}}^{\bar{H}}\left(\psi_{3,2}\right) & =\phi_{3,6}+\phi_{3,10}+\phi_{3,2} & \operatorname{Ind}_{\bar{H}}^{\bar{H}}\left(\psi_{3,4}\right)=\phi_{3,4}+\phi_{3,8}+\phi_{3,12}
\end{array}
$$

Let

$$
\theta: \begin{cases}x_{i} \mapsto\left(\zeta_{2}\right)^{i} q^{a_{i}} & (0 \leq i<2) \\ z_{k} \mapsto\left(\zeta_{3}\right)^{k} q^{c_{k}} & (0 \leq k<3)\end{cases}
$$

be a cyclotomic specialization of $\mathcal{H}\left(G_{6}\right)$. Let us consider the corresponding cyclotomic specialization of $\mathcal{H}\left(G_{7}\right)$

$$
\vartheta: \begin{cases}x_{i} \mapsto\left(\zeta_{2}\right)^{i} q^{a_{i}} & (0 \leq i<2), \\ y_{j} \mapsto\left(\zeta_{3}\right)^{j} & (0 \leq j<3), \\ z_{k} \mapsto\left(\zeta_{3}\right)^{k} q^{c_{k}} & (0 \leq k<3) .\end{cases}
$$

Then $\left(\mathcal{H}\left(G_{7}\right)\right)_{\vartheta}$ is the twisted symmetric algebra of the cyclic group $C_{3}$ over the symmetric subalgebra $\left(\mathcal{H}\left(G_{6}\right)\right)_{\theta}$. Therefore, the essential hyperplanes for $G_{6}$ are obtained from the essential hyperplanes for $G_{7}$ by setting $b_{0}=b_{1}=b_{2}=0$. If now, for example, $\theta$ is associated with no essential hyperplane for $G_{6}$, then the Rouquier blocks of $\left(\mathcal{H}\left(G_{7}\right)\right)_{\vartheta}$ are:

$$
\begin{gathered}
\left\{\phi_{1,0}, \phi_{1,4^{\prime \prime}}, \phi_{1,8^{\prime \prime \prime}}\right\},\left\{\phi_{1,4^{\prime}}, \phi_{1,8^{\prime \prime}}, \phi_{1,12^{\prime \prime}}\right\},\left\{\phi_{1,8^{\prime}}, \phi_{1,12^{\prime}}, \phi_{1,16}\right\}, \\
\left\{\phi_{1,6}, \phi_{1,10^{\prime \prime}}, \phi_{1,14^{\prime \prime \prime}}\right\},\left\{\phi_{1,10^{\prime}}, \phi_{1,14^{\prime \prime}}, \phi_{1,18^{\prime \prime}}\right\},\left\{\phi_{1,14^{\prime}}, \phi_{1,18^{\prime}}, \phi_{1,22}\right\} \\
\left\{\phi_{2,9^{\prime}}, \phi_{2,13^{\prime \prime}}, \phi_{2,5^{\prime \prime \prime}}, \phi_{2,7^{\prime \prime}}, \phi_{2,11^{\prime \prime \prime}}, \phi_{2,15}\right\},\left\{\phi_{2,7^{\prime}}, \phi_{2,11^{\prime \prime}}, \phi_{2,3^{\prime \prime}}, \phi_{2,9^{\prime \prime \prime}}, \phi_{2,13^{\prime}}, \phi_{2,5^{\prime \prime}}\right\} \\
\left\{\phi_{2,11^{\prime}}, \phi_{2,7^{\prime \prime \prime}}, \phi_{2,3^{\prime}}, \phi_{2,9^{\prime \prime}}, \phi_{2,5^{\prime}}, \phi_{2,1}\right\},\left\{\phi_{3,6}, \phi_{3,10}, \phi_{3,2}\right\},\left\{\phi_{3,4}, \phi_{3,8}, \phi_{3,12}\right\} .
\end{gathered}
$$

By Clifford theory, the Rouquier blocks of $\left(\mathcal{H}\left(G_{6}\right)\right)_{\theta}$, i.e., the Rouquier blocks associated with no essential hyperplane for $G_{6}$ are:

$$
\begin{gathered}
\left\{\psi_{1,0}\right\},\left\{\psi_{1,4}\right\},\left\{\psi_{1,8}\right\},\left\{\psi_{1,6}\right\},\left\{\psi_{1,10}\right\},\left\{\psi_{1,14}\right\}, \\
\left\{\psi_{2,5^{\prime \prime}}, \psi_{2,7}\right\},\left\{\psi_{2,3^{\prime \prime}}, \psi_{2,5^{\prime}}\right\},\left\{\psi_{2,3^{\prime}}, \psi_{2,1}\right\},\left\{\psi_{3,2}\right\},\left\{\psi_{3,4}\right\} .
\end{gathered}
$$

In the same way, we obtain the Rouquier blocks associated with each essential hyperplane for $G_{6}$. Here we present only the non-trivial ones:

\section{No essential hyperplane}

$\left\{\psi_{2,5^{\prime \prime}}, \psi_{2,7}\right\},\left\{\psi_{2,3^{\prime \prime}}, \psi_{2,5^{\prime}}\right\},\left\{\psi_{2,3^{\prime}}, \psi_{2,1}\right\}$

$c_{1}-c_{2}=0$

$\left\{\psi_{1,4}, \psi_{1,8}\right\},\left\{\psi_{1,10}, \psi_{1,14}\right\},\left\{\psi_{2,5^{\prime \prime}}, \psi_{2,7}\right\},\left\{\psi_{2,3^{\prime \prime}}, \psi_{2,3^{\prime}}, \psi_{2,1}, \psi_{2,5^{\prime}}\right\}$

$c_{0}-c_{1}=0$

$\left\{\psi_{1,0}, \psi_{1,4}\right\},\left\{\psi_{1,6}, \psi_{1,10}\right\},\left\{\psi_{2,5^{\prime \prime}}, \psi_{2,3^{\prime \prime}}, \psi_{2,7}, \psi_{2,5^{\prime}}\right\},\left\{\psi_{2,3^{\prime}}, \psi_{2,1}\right\}$ 


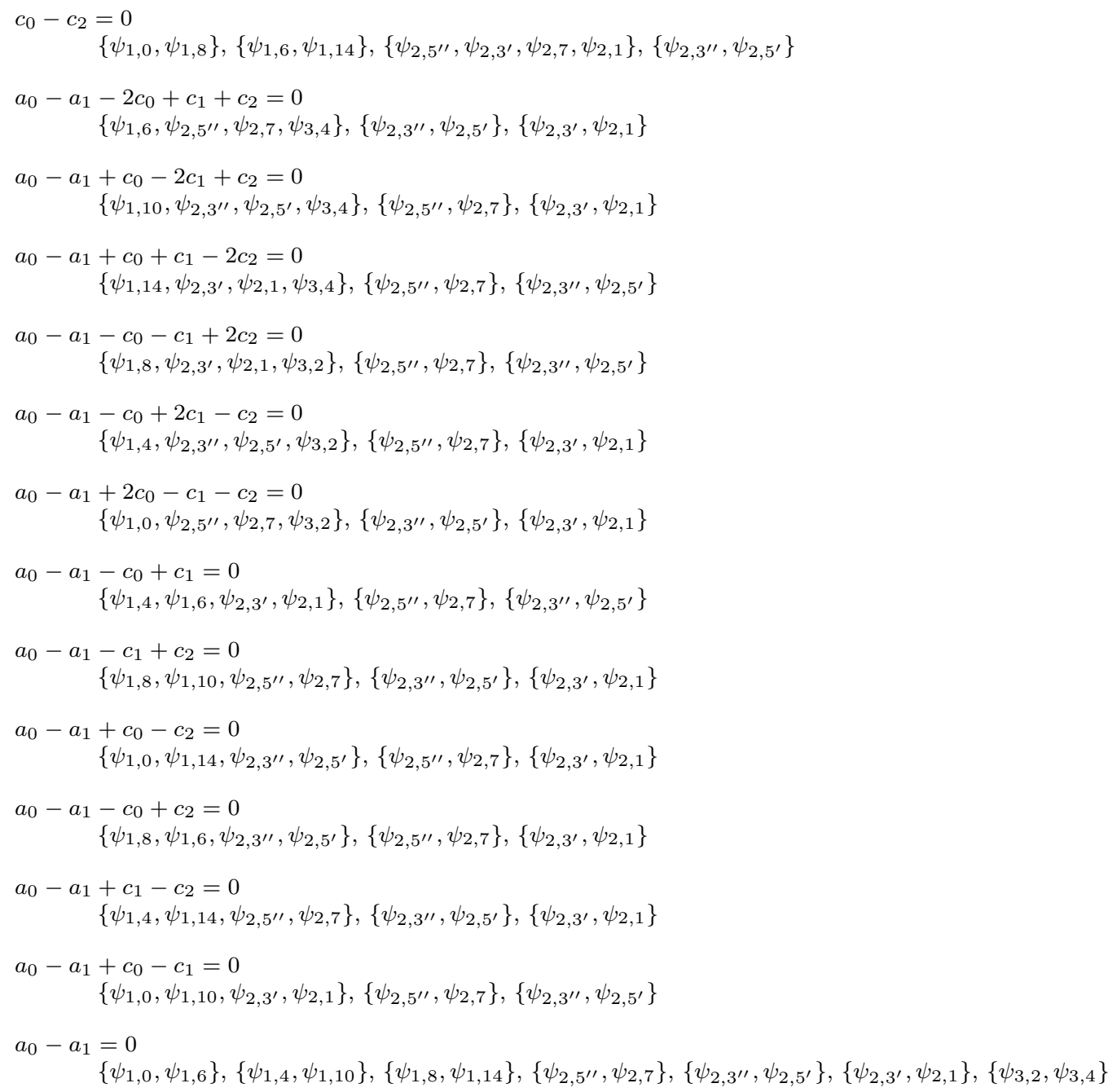

Since it will take too many pages to give here the Rouquier blocks associated with all essential hyperplanes for all exceptional irreducible complex reflection groups, and in order to make it easier to work with them, we have stored these data in a computer file and created two GAP functions which display them. These functions are called AllBlocks and DisplayAllBlocks and they can be found on my webpage, along with explanations for their use. Here is an example of the use of the second one on the group $G_{4}$.

Example 5.2.4 gap> W:=ComplexReflectionGroup(4);

gap> DisplayAllBlocks (W);

No essential hyperplane

[["phi $\{1,0\} "],[" p h i\{1,4\} "],[" p h i\{1,8\} "],[" p h i\{2,5\} "]$, ["phi $\{2,3\} "],[" \operatorname{phi}\{2,1\} "],[" \operatorname{phi}\{3,2\} "]]$ 


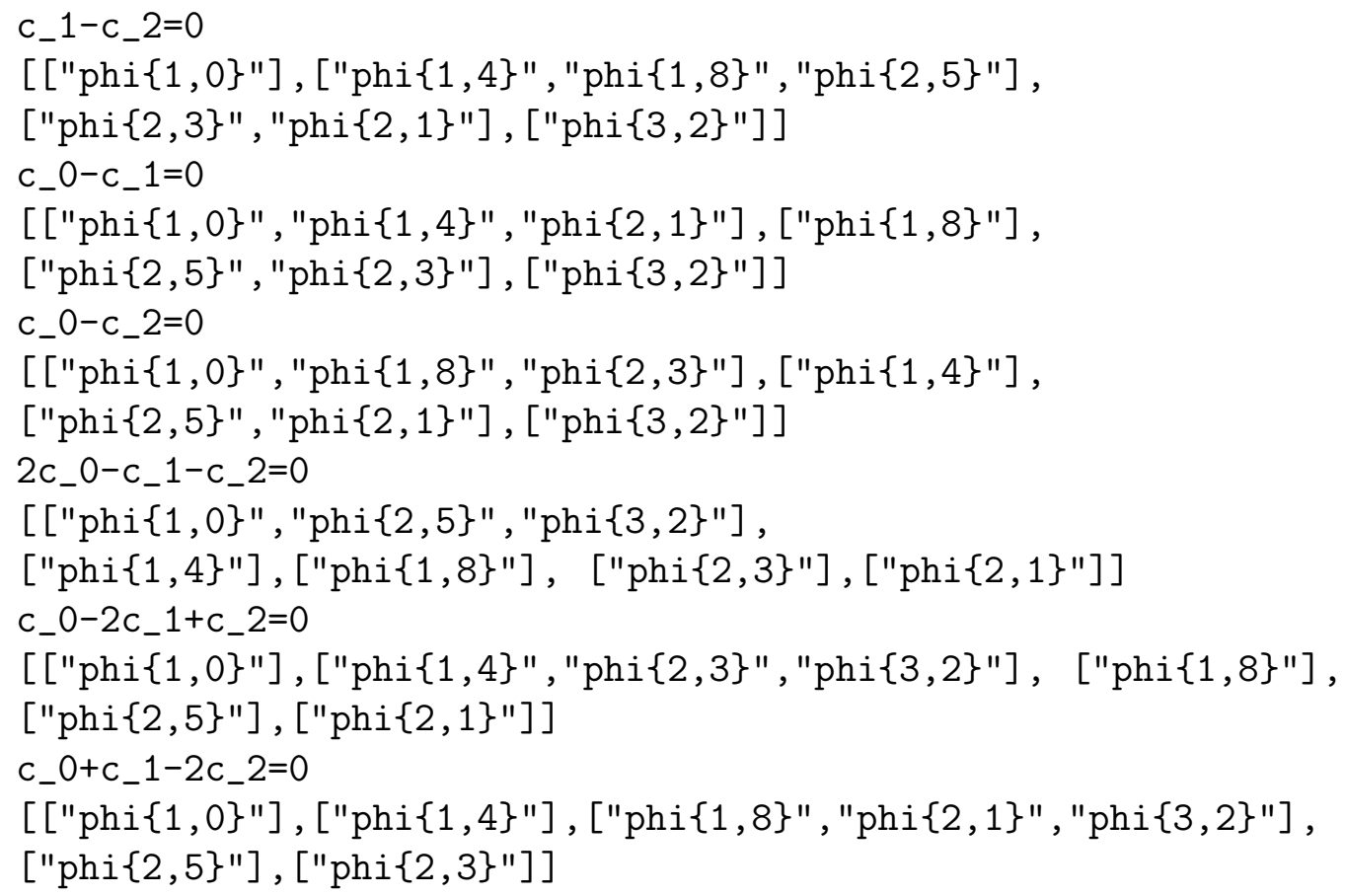

Let $W$ be any exceptional irreducible complex reflection group. Now that we have the Rouquier blocks associated with no and each essential hyperplane for $W$, we can determine the Rouquier blocks of any cyclotomic Hecke algebra associated to $W$ with the use of Theorem 5.1.1. We have also created the GAP functions RouquierBlocks and DisplayRouquierBlocks (corresponding to AllBlocks and DisplayAllBlocks) which, given a cyclotomic specialization $\phi: u_{\mathcal{C}, j} \mapsto \zeta_{e_{\mathcal{C}}}^{j} q^{n_{\mathcal{C}, j}}$, check to which essential hyperplanes the integers $n_{\mathcal{C}, j}$ belong and, using the stored data, apply Theorem 5.1.1 to return the Rouquier blocks of $\mathcal{H}_{\phi}$. We will give here an example of their use on $G_{4}$.

Example 5.2.5 The generic Hecke algebra of $G_{4}$ has a presantation of the form

$$
\begin{aligned}
\mathcal{H}\left(G_{4}\right)=<S, T \mid S T S=T S T, & \left(S-u_{0}\right)\left(S-u_{1}\right)\left(S-u_{2}\right)=0 \\
& \left(T-u_{0}\right)\left(T-u_{1}\right)\left(T-u_{2}\right)=0>
\end{aligned}
$$

If we want to calculate the Rouquier blocks of the cyclotomic Hecke algebra

$$
\begin{array}{ll}
\mathcal{H}_{\phi}=<S, T \mid S T S=T S T, & (S-1)\left(S-\zeta_{3} q\right)\left(S-\zeta_{3}^{2} q^{2}\right)=0 \\
& (T-1)\left(T-\zeta_{3} q\right)\left(T-\zeta_{3}^{2} q^{2}\right)=0>
\end{array}
$$

we use the following commands (the way to define a cyclotomic Hecke algebra in CHEVIE is explained in the GAP manual, cf., for example, [54]): 


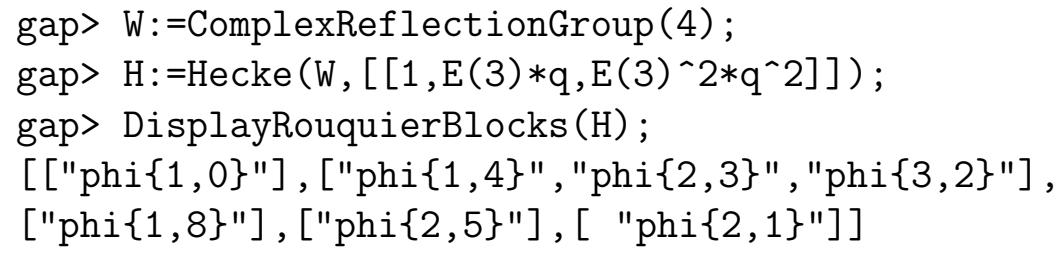

\subsection{The groups $G(d, 1, r)$}

The group $G(d, 1, r)$ is the group of all $r \times r$ monomial matrices with non-zero entries in $\mu_{d}$. It is isomorphic to the wreath product $\mu_{d} \prec \mathfrak{S}_{r}$ and its field of definition is $\mathbb{Q}\left(\zeta_{d}\right)$.

We will start by introducing some notations and results in combinatorics (cf. [18, §3A) which will be useful for the description of the Rouquier blocks of the cyclotomic Ariki-Koike algebras, i.e., the cyclotomic Hecke algebras associated to the group $G(d, 1, r)$.

\subsubsection{Combinatorics}

Let $\lambda=\left(\lambda_{1}, \lambda_{2}, \ldots, \lambda_{h}\right)$ be a partition, i.e., a finite decreasing sequence of positive integers

$$
\lambda_{1} \geq \lambda_{2} \geq \ldots \geq \lambda_{h} \geq 1
$$

The integer

$$
|\lambda|:=\lambda_{1}+\lambda_{2}+\ldots+\lambda_{h}
$$

is called the size of $\lambda$. We also say that $\lambda$ is a partition of $|\lambda|$. The integer $h$ is called the height of $\lambda$ and we set $h_{\lambda}:=h$. To each partition $\lambda$ we associate its $\beta$-number, $\beta_{\lambda}=\left(\beta_{1}, \beta_{2}, \ldots, \beta_{h}\right)$, defined by

$$
\beta_{1}:=h+\lambda_{1}-1, \beta_{2}:=h+\lambda_{2}-2, \ldots, \beta_{h}:=h+\lambda_{h}-h .
$$

Example 5.3.1 If $\lambda=(4,2,2,1)$, then $\beta_{\lambda}=(7,4,3,1)$.

Let $n$ be a non-negative integer. The $n$-shifted $\beta$-number of $\lambda$ is the sequence of numbers defined by

$$
\beta_{\lambda}[n]:=\left(\beta_{1}+n, \beta_{2}+n, \ldots, \beta_{h}+n, n-1, n-2, \ldots, 1,0\right) .
$$

We have $\beta_{\lambda}[0]=\beta_{\lambda}$.

Example 5.3.2 If $\lambda=(4,2,2,1)$, then $\beta_{\lambda}[3]=(10,7,6,4,2,1,0)$. 


\section{Multipartitions}

Let $d$ be a positive integer and let $\lambda=\left(\lambda^{(0)}, \lambda^{(1)}, \ldots, \lambda^{(d-1)}\right)$ be a $d$-partition, i.e., a family of $d$ partitions indexed by the set $\{0,1, \ldots, d-1\}$. We set

$$
h^{(a)}:=h_{\lambda^{(a)}}, \quad \beta^{(a)}:=\beta_{\lambda^{(a)}}
$$

and we have

$$
\lambda^{(a)}=\left(\lambda_{1}^{(a)}, \lambda_{2}^{(a)}, \ldots, \lambda_{h^{(a)}}^{(a)}\right)
$$

The integer

$$
|\lambda|:=\sum_{a=0}^{d-1}\left|\lambda^{(a)}\right|
$$

is called the size of $\lambda$. We also say that $\lambda$ is a d-partition of $|\lambda|$.

\section{Ordinary symbols}

Let $\lambda=\left(\lambda^{(0)}, \lambda^{(1)}, \ldots, \lambda^{(d-1)}\right)$ be a $d$-partition. We call $d$-height of $\lambda$ the family $\left(h^{(0)}, h^{(1)}, \ldots, h^{(d-1)}\right)$ and we define the height of $\lambda$ to be the integer

$$
h_{\lambda}:=\max \left\{h^{(a)} \mid 0 \leq a \leq d-1\right\} .
$$

Definition 5.3.3 The ordinary standard symbol of $\lambda$ is the family of numbers defined by

$$
B_{\lambda}=\left(B_{\lambda}^{(0)}, B_{\lambda}^{(1)}, \ldots, B_{\lambda}^{(d-1)}\right),
$$

where, for all $a(0 \leq a \leq d-1)$, we have

$$
B_{\lambda}^{(a)}:=\beta^{(a)}\left[h_{\lambda}-h^{(a)}\right] .
$$

An ordinary symbol of $\lambda$ is a symbol obtained from the ordinary standard symbol by shifting all the rows by the same integer.

The ordinary standard symbol of a $d$-partition $\lambda$ is of the form

$$
\begin{array}{cccccc}
B_{\lambda}^{(0)} & = & b_{1}^{(0)} & b_{2}^{(0)} & \ldots & b_{h}^{(0)} \\
B_{\lambda}^{(1)} & = & b_{1}^{(1)} & b_{2}^{(1)} & \ldots & b_{h_{\lambda}}^{(1)} \\
\vdots & & \vdots & \vdots & \vdots & \vdots \\
B_{\lambda}^{(d-1)} & = & b_{1}^{(d-1)} & b_{2}^{(d-1)} & \ldots & b_{h_{\lambda}}^{(d-1)}
\end{array}
$$

The ordinary content of a $d$-partition of ordinary standard symbol $B_{\lambda}$ is the multiset

$$
\text { Cont }_{\lambda}:=B_{\lambda}^{(0)} \cup B_{\lambda}^{(1)} \cup \ldots \cup B_{\lambda}^{(d-1)}
$$


or (with the above notations) the polynomial defined by

$$
\operatorname{Cont}_{\lambda}(x):=\sum_{0 \leq a<d} \sum_{1 \leq i \leq h_{\lambda}} x^{b_{i}^{(a)}} .
$$

Example 5.3.4 If $d=2$ and $\lambda=((2,1),(3))$, then

$$
B_{\lambda}=\left(\begin{array}{ll}
3 & 1 \\
4 & 0
\end{array}\right)
$$

We have $\operatorname{Cont}_{\lambda}=\{0,1,3,4\}$ or $\operatorname{Cont}_{\lambda}(x)=1+x+x^{3}+x^{4}$.

\section{Charged symbols}

Let us suppose that we have a given "weight system", i.e., a family of integers

$$
m:=\left(m^{(0)}, m^{(1)}, \ldots, m^{(d-1)}\right)
$$

Let $\lambda=\left(\lambda^{(0)}, \lambda^{(1)}, \ldots, \lambda^{(d-1)}\right)$ be a $d$-partition. We call $(d, m)$-charged height of $\lambda$ the family $\left(h c^{(0)}, h c^{(1)}, \ldots, h c^{(d-1)}\right)$, where

$$
h c^{(0)}:=h^{(0)}-m^{(0)}, h c^{(1)}:=h^{(1)}-m^{(1)}, \ldots, h c^{(d-1)}:=h^{(d-1)}-m^{(d-1)} .
$$

We define the $m$-charged height of $\lambda$ to be the integer

$$
h c_{\lambda}:=\max \left\{h c^{(a)} \mid 0 \leq a \leq d-1\right\} .
$$

Definition 5.3.5 The m-charged standard symbol of $\lambda$ is the family of numbers defined by

$$
B c_{\lambda}=\left(B c_{\lambda}^{(0)}, B c_{\lambda}^{(1)}, \ldots, B c_{\lambda}^{(d-1)}\right),
$$

where, for all $a(0 \leq a \leq d-1)$, we have

$$
B c_{\lambda}^{(a)}:=\beta^{(a)}\left[h c_{\lambda}-h c^{(a)}\right] .
$$

An $m$-charged symbol of $\lambda$ is a symbol obtained from the $m$-charged standard symbol by shifting all the rows by the same integer.

Remark: The ordinary symbols correspond to the weight system

$$
m^{(0)}=m^{(1)}=\ldots=m^{(d-1)}=0 .
$$


The $m$-charged standard symbol of $\lambda$ is a tableau of numbers arranged into $d$ rows indexed by the set $\{0,1, \ldots, d-1\}$ such that the $a^{\text {th }}$ row has length equal to $h c_{\lambda}+m^{(a)}$. For all $a(0 \leq a \leq d-1)$, we set $l^{(a)}:=h c_{\lambda}+m^{(a)}$ and we denote by

$$
B c_{\lambda}^{(a)}=b c_{1}^{(a)} \quad b c_{2}^{(a)} \quad \ldots \quad b c_{l^{(a)}}^{(a)}
$$

the $a^{\text {th }}$ row of the $m$-charged standard symbol.

The $m$-charged content of a $d$-partition of $m$-charged standard symbol $B c_{\lambda}$ is the multiset

$$
\operatorname{Contc}_{\lambda}:=B c_{\lambda}^{(0)} \cup B c_{\lambda}^{(1)} \cup \ldots \cup B c_{\lambda}^{(d-1)}
$$

or (with the above notations) the polynomial defined by

$$
\operatorname{Contc}_{\lambda}(x):=\sum_{0 \leq a<d} \sum_{1 \leq i \leq l^{(a)}} x^{b c_{i}^{(a)}}
$$

Example 5.3.6 If $d=2, \lambda=((2,1),(3))$ and $m=(-1,2)$, then

$$
B c_{\lambda}=\left(\begin{array}{rrrrr}
3 & 1 & & & \\
7 & 3 & 2 & 1 & 0
\end{array}\right)
$$

We have $\operatorname{Contc}_{\lambda}=\{0,1,1,2,3,3,7\}$ or $\operatorname{Contc}_{\lambda}(x)=1+2 x+x^{2}+2 x^{3}+x^{7}$.

\subsubsection{Ariki-Koike algebras}

The generic Ariki-Koike algebra associated to $G(d, 1, r)$ (cf. 4], [19]) is the algebra $\mathcal{H}_{d, r}$ generated over the Laurent polynomial ring in $d+1$ indeterminates

$$
\mathcal{L}_{d}:=\mathbb{Z}\left[u_{0}, u_{0}^{-1}, u_{1}, u_{1}^{-1}, \ldots, u_{d-1}, u_{d-1}^{-1}, x, x^{-1}\right]
$$

by the elements $\mathbf{s}, \mathbf{t}_{1}, \mathbf{t}_{2}, \ldots, \mathbf{t}_{r-1}$ satisfying the relations

- $\mathbf{s t}_{1} \mathbf{s t}_{1}=\mathbf{t}_{1} \mathbf{s t}_{1} \mathbf{s}, \mathbf{s t}_{j}=\mathbf{t}_{j} \mathbf{s}$ for $j \neq 1$,

- $\mathbf{t}_{j} \mathbf{t}_{j+1} \mathbf{t}_{j}=\mathbf{t}_{j+1} \mathbf{t}_{j} \mathbf{t}_{j+1}, \mathbf{t}_{i} \mathbf{t}_{j}=\mathbf{t}_{j} \mathbf{t}_{i}$ for $|i-j|>1$,

- $\left(\mathbf{s}-u_{0}\right)\left(\mathbf{s}-u_{1}\right) \ldots\left(\mathbf{s}-u_{d-1}\right)=0$,

- $\left(\mathbf{t}_{j}-x\right)\left(\mathbf{t}_{j}+1\right)=0$ for all $j=1,2, \ldots, r-1$. 
Remark: If the last relation in the above definition is replaced by

$$
\left(\mathbf{t}_{j}-x\right)\left(\mathbf{t}_{j}-1\right)=0,
$$

then we obtain a presentation of the generic Hecke algebra of $G(d, 1, r)$. However, the second "-" becomes a "+", when we specialize via a cyclotomic specialization, so we might as well consider the generic Ariki-Koike algebra instead.

For every $d$-partition $\lambda$ of $r$, we consider the free $\mathcal{L}_{d}$-module which has as basis the family of standard tableaux of $\lambda$. We can give to this module the structure of a $\mathcal{H}_{d, r}$-module (cf. [4], 3], 38]) and hence obtain the Specht module $\mathbf{S p}^{\lambda}$ associated to $\lambda$.

Let $\mathcal{K}_{d}$ be the field of fractions of $\mathcal{L}_{d}$. The $\mathcal{K}_{d} \mathcal{H}_{d, r}$-module $\mathcal{K}_{d} \mathbf{S p}^{\lambda}$, obtained by extension of scalars, is absolutely irreducible and every irreducible $\mathcal{K}_{d} \mathcal{H}_{d, r}$-module is isomorphic to a module of this type. Thus, $\mathcal{K}_{d}$ is a splitting field for $\mathcal{H}_{d, r}$. We denote by $\chi_{\lambda}$ the (absolutely) irreducible character of the $\mathcal{K}_{d} \mathcal{H}_{d, r}$-module $\mathcal{K}_{d} \mathbf{S p}^{\lambda}$.

\subsubsection{Rouquier blocks, charged content and residues}

Let $q$ be an indeterminate and let

$$
\phi:\left\{\begin{array}{l}
u_{a} \mapsto \zeta_{d}^{a} q^{m_{a}}(0 \leq a<d) \\
x \mapsto q^{n}
\end{array}\right.
$$

be a cyclotomic specialization of $\mathcal{H}_{d, r}$. Since the algebra $\mathcal{K}_{d} \mathcal{H}_{d, r}$ is split, we can deduce easily from Theorem 4.2.4 and Proposition 4.3.4 that the algebra $\mathbb{Q}\left(\zeta_{d}, q\right)\left(\mathcal{H}_{d, r}\right)_{\phi}$ is split semisimple. Therefore, the Rouquier blocks of $\left(\mathcal{H}_{d, r}\right)_{\phi}$ are the blocks of the algebra $\mathcal{R}_{\mathbb{Q}\left(\zeta_{d}\right)}(q)\left(\mathcal{H}_{d, r}\right)_{\phi}$, where

$$
\mathcal{R}_{\mathbb{Q}\left(\zeta_{d}\right)}(q)=\mathbb{Z}\left[\zeta_{d}\right]\left[q, q^{-1},\left(q^{n}-1\right)_{n \geq 1}^{-1}\right] .
$$

Theorem 3.13 in [18] gives a description of the Rouquier blocks of $\left(\mathcal{H}_{d, r}\right)_{\phi}$ when $n \neq 0$. However, in the proof it is supposed that $1-\zeta_{d}$ always belongs to a prime ideal of $\mathbb{Z}\left[\zeta_{d}\right]$. This is not correct, unless $d$ is a power of a prime number. Therefore, we will state here the part of the theorem that is correct and only for the case $n=1$.

Theorem 5.3.7 Let $\phi$ be a cyclotomic specialization of $\mathcal{H}_{d, r}$ such that $\phi(x)=$ $q$. Let $\lambda$ and $\mu$ be two d-partitions of $r$. If the irreducible characters $\left(\chi_{\lambda}\right)_{\phi}$ and $\left(\chi_{\mu}\right)_{\phi}$ are in the same Rouquier block of $\left(\mathcal{H}_{d, r}\right)_{\phi}$, then Contc $_{\lambda}=$ Contc $_{\mu}$ with respect to the weight system $m=\left(m_{0}, m_{1}, \ldots, m_{d-1}\right)$. The converse holds when $d$ is a power of a prime number. 
Remark: In [24], we have proved that the converse of Theorem 5.3.7, and thus the description by Broué and Kim, holds when $\phi$ is the "spetsial" cyclotomic specialization and $d$ is any positive integer.

Set $\mathcal{O}:=\mathcal{R}_{\mathbb{Q}\left(\zeta_{d}\right)}(q)$. Let $\mathfrak{p}$ be a prime ideal of $\mathbb{Z}\left[\zeta_{d}\right]$ lying over a prime number $p$. By Proposition 4.4.2, the ring $\mathcal{O}$ is a Dedekind ring, whence $\mathcal{O}_{\mathfrak{p O}}$ is a discrete valuation ring. Let us denote by $k_{\mathfrak{p}}$ the residue field of $\mathcal{O}_{\mathfrak{p O}}$ and by $\pi_{\mathfrak{p}}$ the canonical surjection $\mathcal{O}_{\mathfrak{p O}} \rightarrow k_{\mathfrak{p}}$. Following Corollary 2.1.14, the morphism $\pi_{\mathfrak{p}}$ induces a block bijection between $\mathcal{O}_{\mathfrak{p O}}\left(\mathcal{H}_{d, r}\right)_{\phi}$ and $k_{\mathfrak{p}}\left(\mathcal{H}_{d, r}\right)_{\phi}$.

Definition 5.3.8 The diagram of a d-partition $\lambda$ is the set

$$
[\lambda]:=\left\{(i, j, a) \mid(0 \leq a \leq d-1)\left(1 \leq i \leq h^{(a)}\right)\left(1 \leq j \leq \lambda_{i}^{(a)}\right)\right\} .
$$

$A$ node of $\lambda$ is any ordered triple $(i, j, a) \in[\lambda]$. The $\mathfrak{p}$-residue of the node $x=(i, j, a)$ with respect to $\phi$ is

$\operatorname{res}_{\mathfrak{p}, \phi}(x):= \begin{cases}\left(\pi_{\mathfrak{p}}(j-i), \pi_{\mathfrak{p}}\left(\phi\left(u_{a}\right)\right)\right), & \text { if } n=0 \text { and } \pi_{\mathfrak{p}}\left(\phi\left(u_{a}\right)\right) \neq \pi_{\mathfrak{p}}\left(\phi\left(u_{b}\right)\right) \text { for } b \neq a, \\ \pi_{\mathfrak{p}}\left(\phi\left(u_{a} x^{j-i}\right)\right), & \text { otherwise. }\end{cases}$

Let $\operatorname{Res}_{\mathfrak{p}, \phi}:=\left\{\operatorname{res}_{\mathfrak{p}, \phi}(x) \mid x \in[\lambda]\right.$ for some $d$-partition $\lambda$ of $\left.r\right\}$ be the set of all possible residues. For any $d$-partition $\lambda$ of $r$ and $f \in \operatorname{Res}_{\mathfrak{p}, \phi}$, we set

$$
C_{f}(\lambda):=\left|\left\{x \in[\lambda] \mid \operatorname{res}_{\mathfrak{p}, \phi}(x)=f\right\}\right| .
$$

Definition 5.3.9 Let $\lambda$ and $\mu$ be two d-partitions of $r$. We say that $\lambda$ and $\mu$ are $\mathfrak{p}$-residue equivalent with respect to $\phi$ if $C_{f}(\lambda)=C_{f}(\mu)$ for all $f \in \operatorname{Res}_{\mathfrak{p}, \phi}$.

Then [47], Theorem 2.13 implies that

Theorem 5.3.10 Let $\lambda$ and $\mu$ be two d-partitions of $r$. The irreducible characters $\left(\chi_{\lambda}\right)_{\phi}$ and $\left(\chi_{\mu}\right)_{\phi}$ are in the same block of $\mathcal{O}_{\mathfrak{p O}}\left(\mathcal{H}_{d, r}\right)_{\phi}$ if and only if $\lambda$ and $\mu$ are $\mathfrak{p}$-residue equivalent with respect to $\phi$.

Corollary 5.3.11 Let $\mathfrak{p}_{1}$ and $\mathfrak{p}_{2}$ be two prime ideals of $\mathbb{Z}\left[\zeta_{d}\right]$ lying over the same prime number $p$. Then the blocks of $\mathcal{O}_{\mathfrak{p}_{1} \mathcal{O}}\left(\mathcal{H}_{d, r}\right)_{\phi}$ coincide with the blocks of $\mathcal{O}_{\mathfrak{p}_{2} \mathcal{O}}\left(\mathcal{H}_{d, r}\right)_{\phi}$.

Proof: $\quad$ Let $\mathfrak{p}$ be a prime ideal of $\mathbb{Z}\left[\zeta_{d}\right]$ lying over $p$ and let $a, b, c, d \in \mathbb{Z}$ such that $0 \leq a \leq b \leq d-1$. We have $\pi_{\mathfrak{p}}\left(\zeta_{d}^{a} q^{c}\right)=\pi_{\mathfrak{p}}\left(\zeta_{d}^{b} q^{d}\right)$ if and only if $c=d$ and $\pi_{\mathfrak{p}}\left(\zeta_{d}^{a}\right)=\pi_{\mathfrak{p}}\left(\zeta_{d}^{b}\right)$. If $\pi_{\mathfrak{p}}\left(\zeta_{d}^{a}\right)=\pi_{\mathfrak{p}}\left(\zeta_{d}^{b}\right)$, then the element $\zeta_{d}^{a}-\zeta_{d}^{b}$ belongs to all the prime ideals lying over $p$. Following the definition of $\mathfrak{p}$-residue, we deduce that two $d$-partitions $\lambda$ and $\mu$ of $r$ are $\mathfrak{p}_{1}$-residue equivalent with respect to $\phi$ if and only if $\lambda$ and $\mu$ are $\mathfrak{p}_{2}$-residue equivalent with respect to $\phi$.

Theorem 5.3.10, in combination with Proposition 4.4.4, gives 
Proposition 5.3.12 Let $\lambda$ and $\mu$ be two d-partitions of $r$. The irreducible characters $\left(\chi_{\lambda}\right)_{\phi}$ and $\left(\chi_{\mu}\right)_{\phi}$ are in the same Rouquier block of $\left(\mathcal{H}_{d, r}\right)_{\phi}$ if and only if there exist a finite sequence $\lambda_{(0)}, \lambda_{(1)}, \ldots, \lambda_{(m)}$ of $d$-partitions of $r$ and a finite sequence $\mathfrak{p}_{1}, \ldots, \mathfrak{p}_{m}$ of $\phi$-bad prime ideals for $G(d, 1, r)$ such that

- $\lambda_{(0)}=\lambda$ and $\lambda_{(m)}=\mu$,

- for all $i(1 \leq i \leq m)$, the $d$-partitions $\lambda_{(i-1)}$ and $\lambda_{(i)}$ are $\mathfrak{p}_{i}$-residue equivalent with respect to $\phi$.

\subsubsection{Essential hyperplanes}

The Schur elements of the algebra $\mathcal{K}_{d} \mathcal{H}_{d, r}$ have been independently calculated by Geck, Iancu and Malle ([35]) and by Mathas ([53]). Following their description by Theorem 6.7.2, we deduce that the essential hyperplanes for $G(d, 1, r)$ are of the form

- $N=0$,

- $k N+M_{s}-M_{t}=0$, where $0 \leq s<t<d$ and $-r<k<r$.

The hyperplane $N=0$ is always essential for $G(d, 1, r)$. Let $H$ be a hyperplane of the form $k N+M_{s}-M_{t}=0$, where $0 \leq s<t<d$ and $-r<k<r$. The hyperplane $H$ is essential for $G(d, 1, r)$ if and only if there exists a prime ideal $\mathfrak{p}$ of $\mathbb{Z}\left[\zeta_{d}\right]$ such that $\zeta_{d}^{s}-\zeta_{d}^{t} \in \mathfrak{p}$. In this case, $H$ is $\mathfrak{p}$-essential for $G(d, 1, r)$. In particular, if $\mathfrak{p}_{1}$ and $\mathfrak{p}_{2}$ are two prime ideals of $\mathbb{Z}\left[\zeta_{d}\right]$ lying over the same prime number $p$, then $H$ is $\mathfrak{p}_{1}$-essential if and only if it is $\mathfrak{p}_{2}$-essential.

Example 5.3.13 The hyperplane $M_{0}=M_{1}$ is (2)-essential for $G(2,1, r)$, whereas it is not essential for $G(6,1, r)$, for all $r>0$.

\subsubsection{Results}

Now we are going to determine the Rouquier blocks associated with no and each essential hyperplane for $G(d, 1, r)$. All the results presented in this section have been first published in [24].

Proposition 5.3.14 The Rouquier blocks associated with no essential hyperplane for $G(d, 1, r)$ are trivial. 
Proof: Let $\phi$ be a cyclotomic specialization assoiciated with no essential hyperplane for $G(d, 1, r)$. By Theorem 6.7.2, the coefficients of the Schur elements of $\mathcal{K}_{d} \mathcal{H}_{d, r}$ are units in $\mathbb{Z}\left[\zeta_{d}\right]$. We deduce that there are no $\phi$-bad prime ideals for $G(d, 1, r)$, whence every irreducible character is a Rouquier block by itself.

Proposition 5.3.15 Let $\lambda, \mu$ be two d-partitions of $r$. The following two assertions are equivalent:

(i) The irreducible characters $\chi_{\lambda}$ and $\chi_{\mu}$ are in the same Rouquier block associated with the essential hyperplane $N=0$.

(ii) We have $\left|\lambda^{(a)}\right|=\left|\mu^{(a)}\right|$ for all $a=0,1, \ldots, d-1$.

Proof: Let

$$
\phi:\left\{\begin{array}{l}
u_{a} \mapsto \zeta_{d}^{a} q^{m_{a}}(0 \leq a<d) \\
x \mapsto 1
\end{array}\right.
$$

be a cyclotomic specialization associated with the essential hyperplane $N=$ 0 .

$(i) \Rightarrow($ ii $)$ Due to Proposition 5.3.12, it is enough to prove that if two $d$ partitions $\lambda, \mu$ of $r$ are $\mathfrak{p}$-residue equivalent with respect to $\phi$ for some prime ideal $\mathfrak{p}$ of $\mathbb{Z}\left[\zeta_{d}\right]$, then $\left|\lambda^{(a)}\right|=\left|\mu^{(a)}\right|$ for all $a=0,1, \ldots, d-1$. Since the integers $m_{a}(0 \leq a<d)$ do not belong to another essential hyperplane for $G(d, 1, r)$, we have $\pi_{\mathfrak{p}}\left(\zeta_{d}^{a} q^{m_{a}}\right) \neq \pi_{\mathfrak{p}}\left(\zeta_{d}^{b} q^{m_{b}}\right)$ for all $0 \leq$ $a<b<d$. If $x=(i, j, a)$ is a node of $\lambda$ or $\mu$, then $\operatorname{res}_{\mathfrak{p}, \phi}(x)=\left(\pi_{\mathfrak{p}}(j-\right.$ $\left.i), \pi_{\mathfrak{p}}\left(\zeta_{d}^{a} q^{m_{a}}\right)\right)$. Since $\lambda$ and $\mu$ are $\mathfrak{p}$-residue equivalent, the number of nodes of $\lambda$ whose $\mathfrak{p}$-residue's second entry is $\pi_{\mathfrak{p}}\left(\zeta_{d}^{a} q^{m_{a}}\right)$ must be equal to the number of nodes of $\mu$ whose $\mathfrak{p}$-residue's second entry is $\pi_{\mathfrak{p}}\left(\zeta_{d}^{a} q^{m_{a}}\right)$, for all $a=0,1, \ldots, d-1$. We deduce that

$$
\begin{aligned}
\left|\lambda^{(a)}\right| & =\left|\left\{(i, j, a) \mid\left(1 \leq i \leq h_{\lambda}^{(a)}\right)\left(1 \leq j \leq \lambda_{i}^{(a)}\right)\right\}\right|= \\
& =\left|\left\{(i, j, a) \mid\left(1 \leq i \leq h_{\mu}^{(a)}\right)\left(1 \leq j \leq \mu_{i}^{(a)}\right)\right\}\right|=\left|\mu^{(a)}\right|
\end{aligned}
$$

for all $a=0,1, \ldots, d-1$.

$(i i) \Rightarrow(i)$ Let $a \in\{0,1, \ldots, d-1\}$. It is enough to show that if $\lambda$ and $\mu$ are two distinct $d$-partitions of $r$ such that

$$
\left|\lambda^{(a)}\right|=\left|\mu^{(a)}\right| \text { and } \lambda^{(b)}=\mu^{(b)} \text { for all } b \neq a,
$$


then $\left(\chi_{\lambda}\right)_{\phi}$ and $\left(\chi_{\mu}\right)_{\phi}$ are in the same Rouquier block of $\left(\mathcal{H}_{d, r}\right)_{\phi}$. Set $l:=\left|\lambda^{(a)}\right|=\left|\mu^{(a)}\right|$. The partitions $\lambda^{(a)}$ and $\mu^{(a)}$ correspond to two distinct irreducible characters of the group $\mathfrak{S}_{l}$. The cyclotomic ArikiKoike algebra obtained from $\mathcal{H}_{1, l}$ via a cyclotomic specialization associated with the hyperplane $N=0$ is isomorphic to the group algebra $\mathbb{Z}\left[\mathfrak{S}_{l}\right]$. For any finite group, it is known that 1 is the only blockidempotent of its group algebra over $\mathbb{Z}$ (see also [57], §3, Rem.1). Thus, all irreducible characters of $\mathfrak{S}_{l}$ belong to the same Rouquier block of $\mathbb{Z}\left[\mathfrak{S}_{l}\right]$. Proposition 5.3 .12 implies that there exist a finite sequence $\nu_{(0)}, \nu_{(1)}, \ldots, \nu_{(m)}$ of partitions of $l$ and a finite sequence $p_{1}, p_{2}, \ldots, p_{m}$ of prime numbers dividing the order of $\mathfrak{S}_{l}$ such that

- $\nu_{(0)}=\lambda^{(a)}$ and $\nu_{(m)}=\mu^{(a)}$,

- for all $i(1 \leq i \leq m), \nu_{(i-1)}$ and $\nu_{(i)}$ are $\left(p_{i}\right)$-residue equivalent with respect to the cyclotomic specialization of $\mathcal{H}_{1, l}$ associated with the essential hyperplane $N=0$.

For all $i(1 \leq i \leq m)$, we define $\nu_{d, i}$ to be the $d$-partition of $r$ such that

$$
\nu_{d, i}^{(a)}:=\lambda_{(i)} \text { and } \nu_{d, i}^{(b)}:=\lambda^{(b)} \text { for all } b \neq a .
$$

Let $\mathfrak{p}_{i}$ be a prime ideal of $\mathbb{Z}\left[\zeta_{d}\right]$ lying over the prime number $p_{i}$. Then we have

- $\nu_{d, 0}=\lambda$ and $\nu_{d, m}=\mu$,

- for all $i(1 \leq i \leq m), \nu_{d, i-1}$ and $\nu_{d, i}$ are $\mathfrak{p}_{i}$-residue equivalent with respect to $\phi$.

By Proposition 5.3 .12 , the characters $\left(\chi_{\lambda}\right)_{\phi}$ and $\left(\chi_{\mu}\right)_{\phi}$ are in the same Rouquier block of $\left(\mathcal{H}_{d, r}\right)_{\phi}$.

Proposition 5.3.16 Let $\lambda, \mu$ be two d-partitions of $r$ and let $H$ be an essential hyperplane for $G(d, 1, r)$ of the form $k N+M_{s}-M_{t}=0$, where $0 \leq s<t<d$ and $-r<k<r$. The irreducible characters $\chi_{\lambda}$ and $\chi_{\mu}$ are in the same Rouquier block associated with the hyperplane $H$ if and only if the following conditions are satisfied:

1. We have $\lambda^{(a)}=\mu^{(a)}$ for all $a \notin\{s, t\}$.

2. If $\lambda^{s t}:=\left(\lambda^{(s)}, \lambda^{(t)}\right)$ and $\mu^{s t}:=\left(\mu^{(s)}, \mu^{(t)}\right)$, then Contc $_{\lambda^{s t}}=$ Contc $_{\mu^{s t}}$ with respect to the weight system $(0, k)$. 
Proof: Let

$$
\phi:\left\{\begin{array}{l}
u_{a} \mapsto \zeta_{d}^{a} q^{m_{a}}(0 \leq j<d) \\
x \mapsto q^{n}
\end{array}\right.
$$

be a cyclotomic specialization associated with the essential hyperplane $H$. We can assume, without loss of generality, that $n=1$. We can also assume that $m_{s}=0$ and $m_{t}=k$.

Suppose that $\left(\chi_{\lambda}\right)_{\phi}$ and $\left(\chi_{\mu}\right)_{\phi}$ belong to the same Rouquier block of $\left(\mathcal{H}_{d, r}\right)_{\phi}$. By Theorem [5.3.7, we have Contc ${ }_{\lambda}=$ Contc $_{\mu}$ with respect to the weight system $m=\left(m_{0}, m_{1}, \ldots, m_{d-1}\right)$. Since the $m_{a}, a \notin\{s, t\}$, can take any value (as long as they do not belong to another essential hyperplane), the equality Contc $_{\lambda}=$ Contc $_{\mu}$ yields the first condition. Moreover, the $\mathrm{m}$ charged standard symbols $B c_{\lambda}$ and $B c_{\mu}$ must have the same cardinality, whence $h c_{\lambda}=h c_{\mu}$. Therefore, we obtain

$$
B c_{\lambda}^{(a)}=\beta_{\lambda}^{(a)}\left[h c_{\lambda}-h c_{\lambda}^{(a)}\right]=\beta_{\mu}^{(a)}\left[h c_{\mu}-h c_{\mu}^{(a)}\right]=B c_{\mu}^{(a)} \text { for all } a \notin\{s, t\},
$$

whence we deduce the following equality between multisets:

$$
B c_{\lambda}^{(s)} \cup B c_{\lambda}^{(t)}=B c_{\mu}^{(s)} \cup B c_{\mu}^{(t)} .
$$

We can assume that the $m_{a}, a \notin\{s, t\}$, are sufficiently large so that

$$
h c_{\lambda} \in\left\{h c_{\lambda}^{(s)}, h c_{\lambda}^{(t)}\right\} \text { and } h c_{\mu} \in\left\{h c_{\mu}^{(s)}, h c_{\mu}^{(t)}\right\} .
$$

If $\lambda^{s t}:=\left(\lambda^{(s)}, \lambda^{(t)}\right)$ and $\mu^{s t}:=\left(\mu^{(s)}, \mu^{(t)}\right)$, then we have

$$
B c_{\lambda^{s t}}^{(0)}=B c_{\lambda}^{(s)}, B c_{\lambda^{s t}}^{(1)}=B c_{\lambda}^{(t)}, B c_{\mu^{s t}}^{(0)}=B c_{\mu}^{(s)}, B c_{\mu^{s t}}^{(1)}=B c_{\mu}^{(t)}
$$

with respect to the weight system $(0, k)$. By definition, we obtain Contc $_{\lambda^{s t}}=$ Contc $_{\mu^{s t}}$ with respect to the weight system $(0, k)$.

Now let us suppose that the conditions 1 and 2 are satisfied. Since $H$ is an essential hyperplane for $G(d, 1, r)$, there exists a prime ideal $\mathfrak{p}$ of $\mathbb{Z}\left[\zeta_{d}\right]$ such that $\zeta_{d}^{s}-\zeta_{d}^{t} \in \mathfrak{p}$. We are going to show that the partitions $\lambda$ and $\mu$ are $\mathfrak{p}$-residue equivalent with respect to $\phi$. Thanks to the first condition, we only need to compare the $\mathfrak{p}$-residues of the nodes with third entry $s$ or $t$.

Set $l:=\left|\lambda^{s t}\right|$. The first condition yields that $\left|\mu^{s t}\right|=l$. Let $\mathcal{H}_{2, l}$ be the generic Ariki-Koike algebra associated to the group $G(2,1, l)$. The algebra $\mathcal{H}_{2, l}$ is defined over the Laurent polynomial ring

$$
\mathbb{Z}\left[U_{0}, U_{0}^{-1}, U_{1}, U_{1}^{-1}, X, X^{-1}\right] .
$$

Let us consider the cyclotomic specialization

$$
\vartheta: U_{0} \mapsto 1, U_{1} \mapsto-q^{k}, X \mapsto q .
$$


Due to Theorem 5.3.7, the condition 2 implies that the characters $\left(\chi_{\lambda^{s t}}\right)_{\vartheta}$ and $\left(\chi_{\mu^{s t}}\right)_{\vartheta}$ belong to the same Rouquier block of $\left(\mathcal{H}_{2, l}\right)_{\vartheta}$. We deduce that $k N+M_{0}-M_{1}=0$ is a (2)-essential hyperplane for $G(2,1, l)$ and that $\vartheta$ is associated with this hyperplane. Following Proposition $5.3 .12, \lambda^{s t}$ and $\mu^{s t}$ must be (2)-residue equivalent with respect to $\vartheta$. We have

- $(i, j, 0) \in\left[\lambda^{s t}\right]$ (resp. $\left.\left[\mu^{s t}\right]\right)$ if and only if $(i, j, s) \in[\lambda]$ (resp. $\left.[\mu]\right)$. Moreover, $\operatorname{res}_{(2), \vartheta}(i, j, 0)=\pi_{(2)}\left(q^{j-i}\right)$, whereas $\operatorname{res}_{\mathfrak{p}, \phi}(i, j, s)=\pi_{\mathfrak{p}}\left(\zeta_{d}^{s} q^{j-i}\right)$.

- $(i, j, 1) \in\left[\lambda^{s t}\right]$ (resp. $\left.\left[\mu^{s t}\right]\right)$ if and only if $(i, j, t) \in[\lambda]$ (resp. $\left.[\mu]\right)$. Moreover, $\operatorname{res}_{(2), \vartheta}(i, j, 1)=\pi_{(2)}\left(-q^{k+j-i}\right)$, whereas $\operatorname{res}_{\mathfrak{p}, \phi}(i, j, s)=\pi_{\mathfrak{p}}\left(\zeta_{d}^{t} q^{k+j-i}\right)$.

Note that we have $\pi_{(2)}(1)=\pi_{(2)}(-1)$ and $\pi_{\mathfrak{p}}\left(\zeta_{d}^{s}\right)=\pi_{\mathfrak{p}}\left(\zeta_{d}^{t}\right)$. We deduce that $\lambda^{s t}$ and $\mu^{s t}$ are (2)-residue equivalent with respect to $\vartheta$ if and only if $\lambda$ and $\mu$ are $\mathfrak{p}$-residue equivalent with respect to $\phi$.

The following result is a corollary of the above proposition. However, we will show that it can also be obtained independently, with the use of the Morita equivalences established in [29].

Corollary 5.3.17 Let $\lambda, \mu$ be two d-partitions of $r$ and let $H$ be an essential hyperplane for $G(d, 1, r)$ of the form $k N+M_{s}-M_{t}=0$, where $0 \leq s<t<d$ and $-r<k<r$. Let

$$
\phi:\left\{\begin{array}{l}
u_{a} \mapsto \zeta_{d}^{a} q^{m_{a}}(0 \leq a<d) \\
x \mapsto q^{n}
\end{array}\right.
$$

be a cyclotomic specialization associated with the essential hyperplane $H$. The irreducible characters $\left(\chi_{\lambda}\right)_{\phi}$ and $\left(\chi_{\mu}\right)_{\phi}$ are in the same Rouquier block of $\left(\mathcal{H}_{d, r}\right)_{\phi}$ if and only if the following conditions are satisfied:

1. We have $\lambda^{(a)}=\mu^{(a)}$ for all $a \notin\{s, t\}$.

2. If $\lambda^{s t}:=\left(\lambda^{(s)}, \lambda^{(t)}\right), \mu^{s t}:=\left(\mu^{(s)}, \mu^{(t)}\right)$ and $l:=\left|\lambda^{s t}\right|=\left|\mu^{s t}\right|$, then the characters $\left(\chi_{\lambda^{s t}}\right)_{\vartheta}$ and $\left(\chi_{\mu^{s t}}\right)_{\vartheta}$ belong to the same Rouquier block of the cyclotomic Ariki-Koike algebra of $G(2,1, l)$ obtained via the specialization

$$
\vartheta: U_{0} \mapsto q^{m_{s}}, U_{1} \mapsto-q^{m_{t}}, X \mapsto q^{n}
$$

Proof: $\quad$ Set $\mathcal{O}:=\mathcal{R}_{\mathbb{Q}\left(\zeta_{d}\right)}(q)$. Since $H$ is an essential hyperplane for $G(d, 1, r)$, there exists a prime ideal $\mathfrak{p}$ of $\mathbb{Z}\left[\zeta_{d}\right]$ such that $\zeta_{d}^{s}-\zeta_{d}^{t} \in \mathfrak{p}$. Due to Corollary 5.3.11, the Rouquier blocks of $\left(\mathcal{H}_{d, r}\right)_{\phi}$ coincide with the blocks of $\mathcal{O}_{\mathfrak{p O}}\left(\mathcal{H}_{d, r}\right)_{\phi}$. 
From now on, all algebras are considered over the ring $\mathcal{O}_{\mathfrak{p O}}$. Following [29], Theorem 1.1, we obtain that the algebra $\left(\mathcal{H}_{d, r}\right)_{\phi}$ is Morita equivalent to the algebra

$$
A:=\bigoplus_{\substack{n_{1}, \ldots, n_{d-1} \geq 0 \\ n_{1}+\ldots+n_{d-1}=r}}\left(\mathcal{H}_{2, n_{1}}\right)_{\phi^{\prime}} \otimes \mathcal{H}\left(\mathfrak{S}_{n_{2}}\right)_{\phi^{\prime \prime}} \otimes \ldots \otimes \mathcal{H}\left(\mathfrak{S}_{n_{d-1}}\right)_{\phi^{\prime \prime}}
$$

where $\phi^{\prime}$ is the restriction of $\phi$ to $\mathbb{Z}\left[u_{s}, u_{s}^{-1}, u_{t}, u_{t}^{-1}, x, x^{-1}\right]$ and $\phi^{\prime \prime}$ is the restriction of $\phi$ to $\mathbb{Z}\left[x, x^{-1}\right]$. Therefore, $\left(\mathcal{H}_{d, r}\right)_{\phi}$ and $A$ have the same blocks.

Since $n \neq 0$, the blocks of $\mathcal{H}\left(\mathfrak{S}_{n_{2}}\right)_{\phi^{\prime \prime}}, \ldots, \mathcal{H}\left(\mathfrak{S}_{n_{2}}\right)_{\phi^{\prime \prime}}$ are trivial. Thus, we obtain that the irreducible characters $\left(\chi_{\lambda}\right)_{\phi}$ and $\left(\chi_{\mu}\right)_{\phi}$ are in the same (Rouquier) block of $\left(\mathcal{H}_{d, r}\right)_{\phi}$ if and only if the following conditions are satisfied:

1. We have $\lambda^{(a)}=\mu^{(a)}$ for all $a \notin\{s, t\}$.

2. If $\lambda^{s t}:=\left(\lambda^{(s)}, \lambda^{(t)}\right), \mu^{s t}:=\left(\mu^{(s)}, \mu^{(t)}\right)$ and $l:=\left|\lambda^{s t}\right|=\left|\mu^{s t}\right|$, then the characters $\left(\chi_{\lambda^{s t}}\right)_{\phi^{\prime}}$ and $\left(\chi_{\mu^{s t}}\right)_{\phi^{\prime}}$ belong to the same block of $\left(\mathcal{H}_{2, l}\right)_{\phi^{\prime}}$.

Theorem 5.3.10 implies that the second condition holds if and only if the 2-partitions $\lambda^{s t}$ and $\mu^{s t}$ are $\mathfrak{p}$-residue equivalent with respect to $\phi^{\prime}$. Using the same argumentation as in the proof of Proposition 5.3.16, we obtain that $\lambda^{s t}$ and $\mu^{s t}$ are $\mathfrak{p}$-residue equivalent with respect to $\phi^{\prime}$ if and only if they are (2)-residue equivalent with respect to $\vartheta$, i.e., if and only if the characters $\left(\chi_{\lambda^{s t}}\right)_{\vartheta}$ and $\left(\chi_{\mu^{s t}}\right)_{\vartheta}$ belong to the same Rouquier block of $\left(\mathcal{H}_{2, l}\right)_{\vartheta}$.

Example 5.3.18 Let $d:=3$ and $r:=3$. The irreducible characters of $G(3,1,3)$ are parametrized by the 3 -partitions of 3 . The generic Ariki-Koike algebra associated to $G(3,1,3)$ is the algebra $\mathcal{H}_{3,3}$ generated over the Laurent polynomial ring in 4 indeterminates

$$
\mathbb{Z}\left[u_{0}, u_{0}^{-1}, u_{1}, u_{1}^{-1}, u_{2}, u_{2}^{-1}, x, x^{-1}\right]
$$

by the elements $\mathbf{s}, \mathbf{t}_{1}, \mathbf{t}_{2}$ satisfying the relations

- $\mathbf{s t}_{1} \mathbf{s t}_{1}=\mathbf{t}_{1} \mathbf{s t}_{1} \mathbf{s}, \mathbf{s t}_{2}=\mathbf{t}_{2} \mathbf{s}, \mathbf{t}_{1} \mathbf{t}_{2} \mathbf{t}_{1}=\mathbf{t}_{2} \mathbf{t}_{1} \mathbf{t}_{2}$,

- $\left(\mathbf{s}-u_{0}\right)\left(\mathbf{s}-u_{1}\right)\left(\mathbf{s}-u_{2}\right)=0$,

- $\left(\mathbf{t}_{1}-x\right)\left(\mathbf{t}_{1}+1\right)=\left(\mathbf{t}_{2}-x\right)\left(\mathbf{t}_{2}+1\right)=0$.

Let

$$
\phi:\left\{\begin{array}{l}
u_{a} \mapsto \zeta_{3}^{a} q^{m_{a}}(0 \leq a \leq 2), \\
x \mapsto q^{n}
\end{array}\right.
$$

be a cyclotomic specialization of $\mathcal{H}_{3,3}$. The essential hyperplanes for $G(3,1,3)$ are: 
- $N=0$.

- $k N+M_{0}-M_{1}=0$ for $k \in\{-2,-1,0,1,2\}$.

- $k N+M_{0}-M_{2}=0$ for $k \in\{-2,-1,0,1,2\}$.

- $k N+M_{1}-M_{2}=0$ for $k \in\{-2,-1,0,1,2\}$.

Let us suppose that $m_{0}=0, m_{1}=0, m_{2}=5$ and $n=1$. These integers belong only to the essential hyperplane $M_{0}-M_{1}=0$. Following Proposition 5.3.16, two irreducible characters $\left(\chi_{\lambda}\right)_{\phi},\left(\chi_{\mu}\right)_{\phi}$ are in the same Rouquier block of $\left(\mathcal{H}_{3,3}\right)_{\phi}$ if and only if

1. We have $\lambda^{(2)}=\mu^{(2)}$.

2. If $\lambda^{01}:=\left(\lambda^{(0)}, \lambda^{(1)}\right)$ and $\mu^{01}:=\left(\mu^{(0)}, \mu^{(1)}\right)$, then Contc $_{\lambda^{01}}=$ Contc $_{\mu^{01}}$ with respect to the weight system $(0,0)$, i.e., Cont $_{\lambda^{01}}=\operatorname{Cont}_{\mu^{01}}$.

The first condition yields immediately that the irreducible characters corresponding to the 3-partitions $(\emptyset, \emptyset,(1,1,1)),(\emptyset, \emptyset,(2,1))$ and $(\emptyset, \emptyset,(3))$ are singletons. Moreover, we have:

$$
\begin{gathered}
B_{((1,1,1), \emptyset)}=\left(\begin{array}{lll}
3 & 2 & 1 \\
2 & 1 & 0
\end{array}\right), B_{(\emptyset,(1,1,1))}=\left(\begin{array}{lll}
2 & 1 & 0 \\
3 & 2 & 1
\end{array}\right), \\
B_{((2,1), \emptyset)}=\left(\begin{array}{ll}
3 & 1 \\
1 & 0
\end{array}\right), B_{(\emptyset,(2,1))}=\left(\begin{array}{ll}
1 & 0 \\
3 & 1
\end{array}\right) \\
B_{((3), \emptyset)}=\left(\begin{array}{l}
3 \\
0
\end{array}\right), B_{(\emptyset,(3))}=\left(\begin{array}{l}
0 \\
3
\end{array}\right) \\
B_{((1,1),(1))}=\left(\begin{array}{ll}
2 & 1 \\
2 & 0
\end{array}\right), B_{((1),(1,1))}=\left(\begin{array}{ll}
2 & 0 \\
2 & 1
\end{array}\right) \\
B_{((2),(1))}=\left(\begin{array}{l}
2 \\
1
\end{array}\right), B_{((1),(2))}=\left(\begin{array}{l}
1 \\
2
\end{array}\right) \\
B_{((1,1), \emptyset)}=\left(\begin{array}{ll}
2 & 1 \\
1 & 0
\end{array}\right), B_{(\emptyset,(1,1))}=\left(\begin{array}{ll}
1 & 0 \\
2 & 1
\end{array}\right) \\
B_{((2), \emptyset)}=\left(\begin{array}{l}
2 \\
0
\end{array}\right), B_{(\emptyset,(2))}=\left(\begin{array}{l}
0 \\
2
\end{array}\right)
\end{gathered}
$$




$$
\begin{gathered}
B_{((1), \emptyset)}=\left(\begin{array}{l}
1 \\
0
\end{array}\right), B_{(\emptyset,(1))}=\left(\begin{array}{l}
0 \\
1
\end{array}\right), \\
B_{((1),(1))}=\left(\begin{array}{l}
1 \\
1
\end{array}\right) .
\end{gathered}
$$

Hence, the Rouquier blocks of $\left(\mathcal{H}_{3,3}\right)_{\phi}$ are:

1. $\left\{\chi_{((1),(1),(1))}\right\}$,

2. $\left\{\chi_{(\emptyset, \emptyset,(1,1,1))}\right\}$,

3. $\left\{\chi_{(\emptyset, \emptyset,(2,1))}\right\}$,

4. $\{\chi(\emptyset, \emptyset,(3))\}$,

5. $\left\{\chi_{((1,1,1), \emptyset, \emptyset)}, \chi_{(\emptyset,(1,1,1), \emptyset)}\right\}$,

6. $\left\{\chi_{((2,1), \emptyset, \emptyset)}, \chi_{(\emptyset,(2,1), \emptyset)}\right\}$,

7. $\left\{\chi_{((3), \emptyset, \emptyset)}, \chi_{(\emptyset,(3), \emptyset)}\right\}$,

8. $\left\{\chi_{((1,1),(1), \emptyset)}, \chi_{((1),(1,1), \emptyset)}\right\}$,

9. $\left\{\chi_{((2),(1), \emptyset)}, \chi_{((1),(2), \emptyset)}\right\}$,

10. $\left\{\chi_{((1,1), \emptyset,(1))}, \chi_{(\emptyset,(1,1),(1))}\right\}$,

11. $\left\{\chi_{((2), \emptyset,(1))}, \chi_{(\emptyset,(2),(1))}\right\}$,

12. $\left\{\chi_{((1), \emptyset,(1,1))}, \chi_{(\emptyset,(1),(1,1))}\right\}$,

13. $\left\{\chi_{((1), \emptyset,(2))}, \chi_{(\emptyset,(1),(2))}\right\}$.

By definition, these are the Rouquier blocks associated with the $\left(1-\zeta_{3}\right)$-essential hyperplane $M_{0}-M_{1}=0$.

If now we take $m_{0}=m_{1}=m_{2}=0$ and $n=1$, then the Rouquier blocks of $\left(\mathcal{H}_{3,3}\right)_{\phi}$ are unions of the Rouquier blocks associated with the essential hyperplanes $M_{0}-M_{1}=0, M_{0}-M_{2}=0$ and $M_{1}-M_{2}=0$. Following Theorem [5.1.1, the Rouquier blocks of $\left(\mathcal{H}_{3,3}\right)_{\phi}$ are:

1. $\left\{\chi_{((1),(1),(1))}\right\}$,

2. $\left\{\chi_{((1,1,1), \emptyset, \emptyset)}, \chi_{(\emptyset,(1,1,1), \emptyset)}, \chi_{(\emptyset, \emptyset,(1,1,1))}\right\}$,

3. $\left\{\chi_{((2,1), \emptyset, \emptyset)}, \chi_{(\emptyset,(2,1), \emptyset)}, \chi_{(\emptyset, \emptyset,(2,1))}\right\}$,

4. $\left\{\chi_{((3), \emptyset, \emptyset)}, \chi_{(\emptyset,(3), \emptyset)}, \chi_{(\emptyset, \emptyset,(3))}\right\}$,

5. $\left\{\chi_{((1,1),(1), \emptyset)}, \chi_{((1),(1,1), \emptyset)}, \chi_{((1,1), \emptyset,(1))}, \chi_{((1), \emptyset,(1,1))}, \chi_{(\emptyset,(1,1),(1))}, \chi_{(\emptyset,(1),(1,1))}\right\}$,

6. $\left\{\chi_{((2),(1), \emptyset)}, \chi_{((1),(2), \emptyset)}, \chi_{((2), \emptyset,(1))}, \chi_{((1), \emptyset,(2))}, \chi_{(\emptyset,(2),(1))}, \chi_{(\emptyset,(1),(2))}\right\}$. 


\subsection{The groups $G(2 d, 2,2)$}

Let $d \geq 1$. The group $G(2 d, 2,2)$ has $4 d$ irreducible characters of degree 1 ,

$$
\chi_{i j k}(0 \leq i, j \leq 1,0 \leq k<d),
$$

and $d^{2}-d$ irreducible characters of degree 2 ,

$$
\chi_{k l}^{1}, \chi_{k l}^{2}(0 \leq k \neq l<d),
$$

where $\chi_{k l}^{1,2}=\chi_{l k}^{1,2}$. The field of definition of $G(2 d, 2,2)$ is $\mathbb{Q}\left(\zeta_{2 d}\right)$.

The generic Hecke algebra of the group $G(2 d, 2,2)$ is the algebra $\mathcal{H}_{2 d}$ generated over the Laurent polynomial ring in $d+4$ indeterminates

$$
\mathbb{Z}\left[x_{0}, x_{0}^{-1}, x_{1}, x_{1}^{-1}, y_{0}, y_{0}^{-1}, y_{1}, y_{1}^{-1}, z_{0}, z_{0}^{-1}, z_{1}, z_{1}^{-1}, \ldots, z_{d-1}, z_{d-1}^{-1}\right]
$$

by the elements $\mathbf{s}, \mathbf{t}, \mathbf{u}$ satisfying the relations

- $\mathbf{s t u}=$ tus $=$ ust

- $\left(\mathbf{s}-x_{0}\right)\left(\mathbf{s}-x_{1}\right)=\left(\mathbf{t}-y_{0}\right)\left(\mathbf{t}-y_{1}\right)=\left(\mathbf{u}-z_{0}\right)\left(\mathbf{u}-z_{1}\right) \ldots\left(\mathbf{u}-z_{d-1}\right)=0$.

\subsubsection{Essential hyperplanes}

Let

$$
\phi: \begin{cases}x_{i} \mapsto(-1)^{i} q^{a_{i}} & (0 \leq i<2) \\ y_{j} \mapsto(-1)^{j} q^{b_{j}} & (0 \leq j<2) \\ z_{k} \mapsto \zeta_{d}^{k} q^{c_{k}} & (0 \leq k<d)\end{cases}
$$

be a cyclotomic specialization of $\mathcal{H}_{2 d}$.

The essential hyperplanes for $G(2 d, 2,2)$ are determined by the Schur elements of $\mathcal{H}_{2 d}$. The Schur elements of $\mathcal{H}_{2 d}$ have been calculated by Malle (48], Theorem 3.11). Following their description (see subsection 6.7.3), the essential hyperplanes for $G(2 d, 2,2)$ are:

- $A_{0}-A_{1}=0 \quad$ (2-essential),

- $B_{0}-B_{1}=0 \quad(2$-essential),

- $C_{k}-C_{l}=0$, where $0 \leq k<l<d$ and $\zeta_{d}^{k}-\zeta_{d}^{l}$ belongs to a prime ideal $\mathfrak{p}$ of $\mathbb{Z}\left[\zeta_{2 d}\right] \quad(\mathfrak{p}$-essential),

- $A_{i}-A_{1-i}+B_{j}-B_{1-j}+C_{k}-C_{l}=0$, where $0 \leq i, j \leq 1,0 \leq k<l<d$ and $\zeta_{d}^{k}-\zeta_{d}^{l}$ belongs to a prime ideal $\mathfrak{p}$ of $\mathbb{Z}\left[\zeta_{2 d}\right]$ (p-essential).

Remark: When we say that a hyperplane is 2-essential, we mean that it is $\mathfrak{I}$-essential for all prime ideals $\mathfrak{I}$ of $\mathbb{Z}\left[\zeta_{2 d}\right]$ lying over 2 . 


\subsubsection{Results}

In order to determine the Rouquier blocks associated with no and each essential hyperplane for $G(2 d, 2,2)$, we are going to use Proposition 4.4.6. Following that result, if two irreducible characters $\chi_{\phi}$ and $\psi_{\phi}$ belong to the same Rouquier block of $\left(\mathcal{H}_{2 d}\right)_{\phi}$, then

$$
a_{\chi_{\phi}}+A_{\chi_{\phi}}=a_{\psi_{\phi}}+A_{\psi_{\phi}} .
$$

Using the formulas for the Schur elements of $\mathcal{H}_{2 d}$ given in the Appendix, we can obtain the value of the sum $a_{\chi_{\phi}}+A_{\chi_{\phi}}$ for all $\chi \in \operatorname{Irr}(G(2 d, 2,2))$ :

Proposition 5.4.1 Let $\chi \in \operatorname{Irr}(G(2 d, 2,2))$. If $\chi$ is a linear character $\chi_{i j k}$, then

$$
a_{\chi_{\phi}}+A_{\chi_{\phi}}=d\left(a_{i}-a_{1-i}+b_{j}-b_{1-j}+2 c_{k}\right)-2 \sum_{l=0}^{d-1} c_{l} .
$$

If $\chi$ is a character $\chi_{k l}^{1,2}$ of degree 2 , then

$$
a_{\chi_{\phi}}+A_{\chi_{\phi}}=d\left(c_{k}+c_{l}\right)-2 \sum_{m=0}^{d-1} c_{m} .
$$

Now we are ready to prove our main result ([25], Theorem 4.3):

Theorem 5.4.2 For the group $G(2 d, 2,2)$, we have that:

1. The non-trivial Rouquier blocks associated with no essential hyperplane are

$$
\left\{\chi_{k l}^{1}, \chi_{k l}^{2}\right\} \text { for all } 0 \leq k<l<d .
$$

2. The non-trivial Rouquier blocks associated with the 2-essential hyperplane $A_{0}=A_{1}$ are

$$
\begin{gathered}
\left\{\chi_{0 j k}, \chi_{1 j k}\right\} \text { for all } 0 \leq j \leq 1 \text { and } 0 \leq k<d, \\
\left\{\chi_{k l}^{1}, \chi_{k l}^{2}\right\} \text { for all } 0 \leq k<l<d .
\end{gathered}
$$

3. The non-trivial Rouquier blocks associated with the 2-essential hyperplane $B_{0}=B_{1}$ are

$$
\begin{gathered}
\left\{\chi_{i 0 k}, \chi_{i 1 k}\right\} \text { for all } 0 \leq i \leq 1 \text { and } 0 \leq k<d \\
\left\{\chi_{k l}^{1}, \chi_{k l}^{2}\right\} \text { for all } 0 \leq k<l<d
\end{gathered}
$$


4. The non-trivial Rouquier blocks associated with the $\mathfrak{p}$-essential hyperplane $C_{k}=C_{l}(0 \leq k<l<d)$ are

$$
\begin{gathered}
\left\{\chi_{i j k}, \chi_{i j l}\right\} \text { for all } 0 \leq i, j \leq 1, \\
\left\{\chi_{k m}^{1}, \chi_{k m}^{2}, \chi_{l m}^{1}, \chi_{l m}^{2}\right\} \text { for all } 0 \leq m<d \text { with } m \notin\{k, l\}, \\
\left\{\chi_{k l}^{1}, \chi_{k l}^{2}\right\}, \\
\left\{\chi_{r s}^{1}, \chi_{r s}^{2}\right\} \text { for all } 0 \leq r<s<d \text { with } r, s \notin\{k, l\} .
\end{gathered}
$$

5. The non-trivial Rouquier blocks associated with the $\mathfrak{p}$-essential hyperplane $A_{i}-A_{1-i}+B_{j}-B_{1-j}+C_{k}-C_{l}=0(0 \leq i, j \leq 1,0 \leq k<l<d)$ are

$$
\begin{gathered}
\left\{\chi_{i j k}, \chi_{1-i, 1-j, l}, \chi_{k l}^{1}, \chi_{k l}^{2}\right\}, \\
\left\{\chi_{r s}^{1}, \chi_{r s}^{2}\right\} \text { for all } 0 \leq r<s<d \text { with }(r, s) \neq(k, l) .
\end{gathered}
$$

Proof: Let

$$
\phi: \begin{cases}x_{i} \mapsto(-1)^{i} q^{a_{i}} & (0 \leq i<2) \\ y_{j} \mapsto(-1)^{j} q^{b_{j}} & (0 \leq j<2) \\ z_{k} \mapsto \zeta_{d}^{k} q^{c_{k}} & (0 \leq k<d)\end{cases}
$$

be a cyclotomic specialization of $\mathcal{H}_{2 d}$.

1. If $\phi$ is a cyclotomic specialization associated with no essential hyperplane, then, by Proposition 2.4.18, each linear character is a Rouquier block by itself, whereas any character of degree 2 is not. Due to the formulas of Proposition 5.4.1, Proposition 4.4.6 yields that the character $\chi_{k l}^{1}(0 \leq k<l<d)$ can be in the same Rouquier block only with the character $\chi_{k l}^{2}$.

2. Suppose that $\phi$ is a cyclotomic specialization associated with the essential hyperplane $A_{0}=A_{1}$. Since the hyperplane $A_{0}=A_{1}$ is not essential for the characters of degree 2, Proposition 3.2.5 implies that $\left\{\chi_{k l}^{1}, \chi_{k l}^{2}\right\}$ is a Rouquier block of $\left(\mathcal{H}_{2 d}\right)_{\phi}$ for all $0 \leq k<l<d$. Moreover, the hyperplane $A_{0}=A_{1}$ is 2-essential for all characters of degree 1 and thus, due to Proposition 2.4.18, there exist no linear character which is a block by itself. Due to the formulas of Proposition 5.4.1, Proposition 4.4 .6 yields that the character $\chi_{0 j k}(0 \leq j \leq 1,0 \leq k<d)$ can be in the same Rouquier block only with the character $\chi_{1 j k}$.

3. If $\phi$ is a cyclotomic specialization associated with the essential hyperplane $B_{0}=B_{1}$, we proceed as in the previous case. 
4. If $\phi$ is a cyclotomic specialization associated with the p-essential hyperplane $C_{k}=C_{l}$, where $0 \leq k<l<d$, then the Rouquier blocks of $\left(\mathcal{H}_{2 d}\right)_{\phi}$ are unions of the Rouquier blocks associated with no essential hyperplane, due to Proposition 3.2.3. Hence, the characters $\chi_{r s}^{1}$ and $\chi_{r s}^{2}$ are in the same Rouquier block of $\left(\mathcal{H}_{2 d}\right)_{\phi}$ for all $0 \leq r<s<d$. Now, the hyperplane $C_{k}=C_{l}$ is $\mathfrak{p}$-essential for the following characters:

- $\chi_{i j k}, \chi_{i j l}$, for all $0 \leq i, j \leq 1$,

- $\chi_{k m}^{1,2}, \chi_{l m}^{1,2}$, for all $0 \leq m<d$ with $m \notin\{k, l\}$.

Due to the formulas of Proposition 5.4.1, Proposition 4.4.6 yields that

- the character $\chi_{i j k}(0 \leq i, j \leq 1)$ can be in the same Rouquier block only with the character $\chi_{i j l}$,

- the character $\chi_{k m}^{1}(0 \leq m<d$ with $m \notin\{k, l\})$ can be in the same Rouquier block only with the characters $\chi_{k m}^{2}, \chi_{l m}^{1}, \chi_{l m}^{2}$.

It remains to show that $\left\{\chi_{k m}^{1}, \chi_{k m}^{2}\right\}(0 \leq m<d$ with $m \notin\{k, l\})$ is not a Rouquier block of $\left(\mathcal{H}_{2 d}\right)_{\phi}$. Following [48], Table 3.10, there exists an element $T_{1}$ of $\mathcal{H}_{2 d}$ such that

$$
\chi_{k m}^{1}\left(T_{1}\right)=\chi_{k m}^{2}\left(T_{1}\right)=x_{0}+x_{1} .
$$

Suppose that $\left\{\chi_{k m}^{1}, \chi_{k m}^{2}\right\}$ is a Rouquier block of $\left(\mathcal{H}_{2 d}\right)_{\phi}$ and set $y^{\left|\mu\left(\mathbb{Q}\left(\zeta_{2 d}\right)\right)\right|}:=$ $q$. Then, by Corollary 2.2.13, we must have

$$
\frac{\phi\left(\chi_{k m}^{1}\left(T_{1}\right)\right)}{\phi\left(s_{\chi_{k m}^{1}}\right)}+\frac{\phi\left(\chi_{k m}^{2}\left(T_{1}\right)\right)}{\phi\left(s_{\chi_{k m}^{2}}\right)} \in \mathcal{O}
$$

where $\mathcal{O}$ denotes the Rouquier ring of $\mathbb{Q}\left(\zeta_{2 d}\right)$. We have

$$
\frac{\phi\left(\chi_{k m}^{1}\left(T_{1}\right)\right)}{\phi\left(s_{\chi_{k m}^{1}}\right)}+\frac{\phi\left(\chi_{k m}^{2}\left(T_{1}\right)\right)}{\phi\left(s_{\chi_{k m}^{2}}\right)}=\phi\left(x_{0}+x_{1}\right) \cdot\left(\frac{1}{\phi\left(s_{\chi_{k m}^{1}}\right)}+\frac{1}{\phi\left(s_{\chi_{k m}^{2}}\right)}\right),
$$

where

$$
\phi\left(x_{0}+x_{1}\right)=q^{a_{0}}-q^{a_{1}}=y^{a_{0}\left|\mu\left(\mathbb{Q}\left(\zeta_{2 d}\right)\right)\right|}-y^{a_{1}\left|\mu\left(\mathbb{Q}\left(\zeta_{2 d}\right)\right)\right|} .
$$

Since $\phi$ is associated with the hyperplane $C_{k}=C_{l}$, we must have $a_{0} \neq a_{1}$, whence $\phi\left(x_{0}+x_{1}\right)^{-1} \in \mathcal{O}$. We deduce that

$$
\frac{1}{\phi\left(s_{\chi_{k m}^{1}}\right)}+\frac{1}{\phi\left(s_{\chi_{k m}^{2}}\right)} \in \mathcal{O} \text {. }
$$

Using the formulas for the description of the Schur elements of $\chi_{k m}^{1,2}$ given in the Appendix, we can easily calculate that the above element does not belong to the Rouquier ring. 
5. Suppose that $\phi$ is a cyclotomic specialization associated with the $\mathfrak{p}$ essential hyperplane $A_{i}-A_{1-i}+B_{j}-B_{1-j}+C_{k}-C_{l}=0$, where $0 \leq i, j \leq 1$ and $0 \leq k<l<d$. We have to distinguish two cases:

(a) If $\mathfrak{p}$ is lying over an odd prime number, then this hyperplane is $\mathfrak{p}$-essential for only three characters: $\chi_{i j k}, \chi_{1-i, 1-j, l}$ and either $\chi_{k l}^{1}$ or $\chi_{k l}^{2}$. If $\mathcal{O}$ is the Rouquier ring of $\mathbb{Q}\left(\zeta_{2 d}\right)$, then, by Proposition 2.4.18, these three characters belong to the same block of $\mathcal{O}_{\mathfrak{p O}}\left(\mathcal{H}_{2 d}\right)_{\phi}$. All the remaining characters are blocks of $\mathcal{O}_{\mathfrak{p O}}\left(\mathcal{H}_{2 d}\right)_{\phi}$ by themselves. Since the Rouquier blocks of $\left(\mathcal{H}_{2 d}\right)_{\phi}$ are unions of the Rouquier blocks associated with no essential hyperplane, we obtain the desired result.

(b) If $\mathfrak{p}$ is lying over 2 , then the hyperplane $A_{i}-A_{1-i}+B_{j}-B_{1-j}+$ $C_{k}-C_{l}=0$ is $\mathfrak{p}$-essential for the characters $\chi_{i j k}, \chi_{1-i, 1-j, l}, \chi_{k l}^{1}$ and $\chi_{k l}^{2}$. Using the same argumentation as in case 4 , we can show that the set $\left\{\chi_{i j k}, \chi_{1-i, 1-j, l}, \chi_{k l}^{1}, \chi_{k l}^{2}\right\}$ is a Rouquier block of $\left(\mathcal{H}_{2 d}\right)_{\phi}$ (and not a union of two Rouquier blocks). Due to Proposition 3.2.5, the remaining Rouquier blocks associated with no essential hyperplane remain as they are.

Example 5.4.3 Let $d:=2$. The group $G(4,2,2)$ has 8 irreducible characters of degree $1, \chi_{i j k}(0 \leq i, j, k \leq 1)$, and 2 irreducible characters of degree $2, \chi_{01}^{1,2}$. The generic Hecke algebra of the group $G(4,2,2)$ is the algebra $\mathcal{H}_{4}$ generated over the Laurent polynomial ring in 6 indeterminates

$$
\mathbb{Z}\left[x_{0}, x_{0}^{-1}, x_{1}, x_{1}^{-1}, y_{0}, y_{0}^{-1}, y_{1}, y_{1}^{-1}, z_{0}, z_{0}^{-1}, z_{1}, z_{1}^{-1}\right]
$$

by the elements $\mathbf{s}, \mathbf{t}, \mathbf{u}$ satisfying the relations

- $\mathbf{s t u}=$ tus $=$ ust

- $\left(\mathbf{s}-x_{0}\right)\left(\mathbf{s}-x_{1}\right)=\left(\mathbf{t}-y_{0}\right)\left(\mathbf{t}-y_{1}\right)=\left(\mathbf{u}-z_{0}\right)\left(\mathbf{u}-z_{1}\right)=0$.

Let

$$
\phi: \begin{cases}x_{i} \mapsto(-1)^{i} q^{a_{i}} & (0 \leq i<2) \\ y_{j} \mapsto(-1)^{j} q^{b_{j}} & (0 \leq j<2) \\ z_{k} \mapsto(-1)^{k} q^{c_{k}} & (0 \leq k<2)\end{cases}
$$

be a cyclotomic specialization of $\mathcal{H}_{4}$. The essential hyperplanes for $G(4,2,2)$ are:

- $H_{1}: A_{0}=A_{1}$,

- $H_{2}: B_{0}=B_{1}$,

- $H_{3}: C_{0}=C_{1}$, 
- $H_{4}: A_{0}-A_{1}+B_{0}-B_{1}+C_{0}-C_{1}=0$.

- $H_{5}: A_{0}-A_{1}+B_{1}-B_{0}+C_{0}-C_{1}=0$.

- $H_{6}: A_{1}-A_{0}+B_{0}-B_{1}+C_{0}-C_{1}=0$.

- $H_{7}: A_{1}-A_{0}+B_{1}-B_{0}+C_{0}-C_{1}=0$.

The only non-trivial Rouquier block associated with no essential hyperplane is $\left\{\chi_{01}^{1}, \chi_{01}^{2}\right\}$. The Rouquier blocks associated with

- $H_{1}$ are: $\left\{\chi_{000}, \chi_{100}\right\},\left\{\chi_{001}, \chi_{101}\right\},\left\{\chi_{010}, \chi_{110}\right\},\left\{\chi_{011}, \chi_{111}\right\},\left\{\chi_{01}^{1}, \chi_{01}^{2}\right\}$.

- $H_{2}$ are: $\left\{\chi_{000}, \chi_{010}\right\},\left\{\chi_{001}, \chi_{011}\right\},\left\{\chi_{100}, \chi_{110}\right\},\left\{\chi_{101}, \chi_{111}\right\},\left\{\chi_{01}^{1}, \chi_{01}^{2}\right\}$.

- $H_{3}$ are: $\left\{\chi_{000}, \chi_{001}\right\},\left\{\chi_{010}, \chi_{011}\right\},\left\{\chi_{100}, \chi_{101}\right\},\left\{\chi_{110}, \chi_{111}\right\},\left\{\chi_{01}^{1}, \chi_{01}^{2}\right\}$.

- $H_{4}$ are: $\left\{\chi_{001}\right\},\left\{\chi_{010}\right\},\left\{\chi_{011}\right\},\left\{\chi_{100}\right\},\left\{\chi_{101}\right\},\left\{\chi_{110}\right\},\left\{\chi_{000}, \chi_{111}, \chi_{01}^{1}, \chi_{01}^{2}\right\}$.

- $H_{5}$ are: $\left\{\chi_{000}\right\},\left\{\chi_{001}\right\},\left\{\chi_{011}\right\},\left\{\chi_{100}\right\},\left\{\chi_{110}\right\},\left\{\chi_{111}\right\},\left\{\chi_{010}, \chi_{101}, \chi_{01}^{1}, \chi_{01}^{2}\right\}$.

- $H_{6}$ are: $\left\{\chi_{000}\right\},\left\{\chi_{001}\right\},\left\{\chi_{010}\right\},\left\{\chi_{101}\right\},\left\{\chi_{110}\right\},\left\{\chi_{111}\right\},\left\{\chi_{100}, \chi_{011}, \chi_{01}^{1}, \chi_{01}^{2}\right\}$.

- $H_{7}$ are: $\left\{\chi_{000}\right\},\left\{\chi_{010}\right\},\left\{\chi_{011}\right\},\left\{\chi_{100}\right\},\left\{\chi_{101}\right\},\left\{\chi_{111}\right\},\left\{\chi_{110}, \chi_{001}, \chi_{01}^{1}, \chi_{01}^{2}\right\}$.

Let us take $a_{0}=2, a_{1}=4, b_{0}=3, b_{1}=1$ and $c_{0}=c_{1}=0$. These integers belong to the essential hyperplanes $H_{3}, H_{4}$ and $H_{7}$. By Theorem 5.1.1, the Rouquier blocks of $\left(\mathcal{H}_{4}\right)_{\phi}$ are

$\left\{\chi_{000}, \chi_{001}, \chi_{110}, \chi_{111}, \chi_{01}^{1}, \chi_{01}^{2}\right\},\left\{\chi_{010}, \chi_{011}\right\},\left\{\chi_{100}, \chi_{101}\right\}$.

\subsection{The groups $G(d e, e, r)$}

All the results in this section have first appeared in [25].

\subsubsection{The groups $G(d e, e, r), r>2$}

We define the Hecke algebra of $G(d e, e, r), r>2$, to be the algebra $\mathcal{H}_{d e, e, r}$ generated over the Laurent polynomial ring in $d+1$ indeterminates

$$
\mathbb{Z}\left[v_{0}, v_{0}^{-1}, v_{1}, v_{1}^{-1}, \ldots, v_{d-1}, v_{d-1}^{-1}, x, x^{-1}\right]
$$

by the elements $a_{0}, a_{1}, \ldots, a_{r}$ satisfying the relations

- $\left(a_{0}-v_{0}\right)\left(a_{0}-v_{1}\right) \ldots\left(a_{0}-v_{d-1}\right)=\left(a_{j}-x\right)\left(a_{j}+1\right)=0$ for $j=1, \ldots, r$,

- $a_{1} a_{3} a_{1}=a_{3} a_{1} a_{3}, a_{j} a_{j+1} a_{j}=a_{j+1} a_{j} a_{j+1}$ for $j=2, \ldots, r-1$, 
- $a_{1} a_{2} a_{3} a_{1} a_{2} a_{3}=a_{3} a_{1} a_{2} a_{3} a_{1} a_{2}$,

- $a_{1} a_{j}=a_{j} a_{1}$ for $j=4, \ldots, r$,

- $a_{i} a_{j}=a_{j} a_{i}$ for $2 \leq i<j \leq r$ with $j-i>1$,

- $a_{0} a_{j}=a_{j} a_{0}$ for $j=3, \ldots, r$,

- $a_{0} a_{1} a_{2}=a_{1} a_{2} a_{0}$,

- $\underbrace{a_{2} a_{0} a_{1} a_{2} a_{1} a_{2} a_{1} \ldots}_{e+1 \text { factors }}=\underbrace{a_{0} a_{1} a_{2} a_{1} a_{2} a_{1} a_{2} \ldots}_{e+1 \text { factors }}$.

Let

$$
\phi:\left\{\begin{array}{l}
v_{j} \mapsto \zeta_{d}^{j} q^{n_{j}}(0 \leq j<d), \\
x \mapsto q^{n}
\end{array}\right.
$$

be a cyclotomic specialization of $\mathcal{H}_{d e, e, r}$. Following Theorem 5.1.1, the Rouquier blocks of $\left(\mathcal{H}_{d e, e, r}\right)_{\phi}$ coincide with the Rouquier blocks of $\left(\mathcal{H}_{d e, e, r}\right)_{\phi^{e}}$, where

$$
\phi^{e}:\left\{\begin{array}{l}
v_{j} \mapsto \zeta_{d}^{j} q^{e n_{j}}(0 \leq j<d) \\
x \mapsto q^{e n}
\end{array}\right.
$$

since the integers $\left\{\left(n_{j}\right)_{0 \leq j<d}, n\right\}$ and $\left\{\left(e n_{j}\right)_{0 \leq j<d}, e n\right\}$ belong to the same essential hyperplanes for $G(d e, e, r)$.

We now consider the generic Ariki-Koike algebra $\mathcal{H}_{d e, r}$ generated over the ring

$$
\mathbb{Z}\left[u_{0}, u_{0}^{-1}, u_{1}, u_{1}^{-1}, \ldots, u_{d e-1}, u_{d e-1}^{-1}, x, x^{-1}\right]
$$

by the elements $\mathbf{s}, \mathbf{t}_{1}, \mathbf{t}_{2}, \ldots, \mathbf{t}_{r-1}$ satisfying the relations described in subsection 5.3.2. Let us consider the following cyclotomic specialization of $\mathcal{H}_{d e, r}$ :

$$
\vartheta:\left\{\begin{array}{l}
u_{a} \mapsto \zeta_{d e}^{a} q^{m_{a}}\left(0 \leq a<d e, m_{a}:=n_{a \bmod d}\right), \\
x \mapsto q^{e n} .
\end{array}\right.
$$

Following Lemma 6.7.1, the algebra $\left(\mathcal{H}_{d e, r}\right)_{\vartheta}$ is the twisted symmetric algebra of the cyclic group $C_{e}$ over the symmetric subalgebra $\left(\mathcal{H}_{d e, e, r}\right)_{\phi^{e}}$.

From now on, set $\mathcal{H}:=\left(\mathcal{H}_{d e, r}\right)_{\vartheta}, \overline{\mathcal{H}}:=\left(\mathcal{H}_{d e, e, r}\right)_{\phi^{e}}, G:=C_{e}, K:=\mathbb{Q}\left(\zeta_{d e}\right)$ and let $\mathcal{R}_{K}(q)$ be the Rouquier ring of $K$. Applying Proposition 2.3 .18 gives:

Proposition 5.5.1 The block-idempotents of $\left(Z \mathcal{R}_{K}(q) \overline{\mathcal{H}}\right)^{G}$ coincide with the block-idempotents of $\left(Z \mathcal{R}_{K}(q) \mathcal{H}\right)^{G^{\vee}}$. 
The action of the cyclic group $G^{\vee}$ of order $e$ on $\operatorname{Irr}(K(q) \mathcal{H})$ corresponds to the action generated by the cyclic permutation by $d$-packages on the $d e-$ partitions of $r$ (cf., for example, [49], §4.A):

$$
\begin{aligned}
\tau_{d}: & \left(\lambda^{(0)}, \ldots, \lambda^{(d-1)}, \lambda^{(d)}, \ldots, \lambda^{(2 d-1)}, \ldots, \lambda^{(d e-d)}, \ldots, \lambda^{(d e-1)}\right) \\
& \mapsto\left(\lambda^{(d e-d)}, \ldots, \lambda^{(d e-1)}, \lambda^{(0)}, \ldots, \lambda^{(d-1)}, \ldots, \lambda^{(d e-2 d)}, \ldots, \lambda^{(d e-d-1)}\right) .
\end{aligned}
$$

The de-partitions which are fixed by the action of $G^{\vee}$, i.e., the de-partitions which are of the form

$$
\left(\lambda^{(0)}, \ldots, \lambda^{(d-1)}, \lambda^{(0)}, \ldots, \lambda^{(d-1)}, \ldots, \lambda^{(0)}, \ldots, \lambda^{(d-1)}\right),
$$

where the first $d$ partitions are repeated $e$ times, are called $d$-stuttering.

Proposition 5.5.2 If $\lambda$ is a de-partition of $r$, then the characters $\chi_{\lambda}$ and $\chi_{\tau_{d}(\lambda)}$ belong to the same Rouquier block of $\mathcal{H}$. In particular, the blocks of $\mathcal{R}_{K}(q) \mathcal{H}$ are stable under the action of $G^{\vee}$.

Proof: The symmetric group $\mathfrak{S}_{d e}$ acts naturally on the set of $d e$-partitions of $r$, and thus on $\operatorname{Irr}(K(q) \mathcal{H})$ : If $\tau \in \mathfrak{S}_{d e}$ and $\lambda=\left(\lambda^{(0)}, \lambda^{(1)}, \ldots, \lambda^{(d e-1)}\right)$ is a de-partition of $r$, then $\tau(\lambda):=\left(\lambda^{(\tau(0))}, \lambda^{(\tau(1))}, \ldots, \lambda^{(\tau(d e-1))}\right)$. The action of $G^{\vee}$ on $\operatorname{Irr}(K(q) \mathcal{H})$ corresponds to the action of the cyclic subgroup of order $e$ of $\mathfrak{S}_{d e}$ generated by the element

$$
\tau_{d}=\prod_{j=0}^{d-1} \prod_{i=1}^{e-1} \sigma_{j, i}
$$

where $\sigma_{j, i}$ denotes the transposition $(j, j+i d)$. In order to prove that the characters $\chi_{\lambda}$ and $\chi_{\tau_{d}(\lambda)}$ belong to the same Rouquier block of $\mathcal{H}$, it suffices to show that the characters $\chi_{\lambda}$ and $\chi_{\sigma_{j, i}(\lambda)}$ are in the same Rouquier block of $\mathcal{H}$ for all $j(0 \leq j<d)$ and $i(0 \leq i<e)$.

Following Theorem 5.1.1, the Rouquier blocks of $\mathcal{H}$ are unions of the Rouquier blocks associated with all the essential hyperplanes of the form

$$
M_{s}=M_{t}(0 \leq s<t<d e, s \equiv t \bmod d) .
$$

Recall that the hyperplane $M_{s}=M_{t}$ is actually essential for $G(d e, 1, r)$ if and only if the element $\zeta_{d e}^{s}-\zeta_{d e}^{t}$ belongs to a prime ideal of $\mathbb{Z}\left[\zeta_{d e}\right]$.

Suppose that $e=p_{1}^{a_{1}} p_{2}^{a_{2}} \ldots p_{m}^{a_{m}}$, where the $p_{k}$ are distinct prime numbers. For $k \in\{1,2, \ldots, m\}$, we set $c_{k}:=e / p_{k}^{a_{k}}$. Then $\operatorname{gcd}\left(c_{k}\right)=1$ and by Bezout's theorem, there exist integers $\left(b_{k}\right)_{1 \leq k \leq m}$ such that $\sum_{k=1}^{m} b_{k} c_{k}=1$. We have 
$i=\sum_{k=1}^{m} i_{k}$, where $i_{k}:=i b_{k} c_{k}$. The element $1-\zeta_{e}^{i_{k}}$ belongs to all the prime ideals of $\mathbb{Z}\left[\zeta_{d e}\right]$ lying over the prime number $p_{k}$. Now set

$$
l_{0}:=0 \text { and } l_{k}:=\left(l_{k-1}+i_{k}\right) \bmod e \text {, for all } k(1 \leq k \leq m) .
$$

We have that the element $\zeta_{d e}^{j+l_{k-1} d}-\zeta_{d e}^{j+l_{k} d}=\zeta_{d e}^{j+l_{k-1} d}\left(1-\zeta_{e}^{i_{k}}\right)$ belongs to all the prime ideals of $\mathbb{Z}\left[\zeta_{d e}\right]$ lying over the prime number $p_{k}$. Therefore, the hyperplane $M_{j+l_{k-1} d}=M_{j+l_{k} d}$ is essential for $G(d e, 1, r)$ for all $k(1 \leq k \leq m)$. Moreover, if we denote by $\tau_{j, i, k}$ the transposition $\left(j+l_{k-1} d, j+l_{k} d\right)$, then

- we have $\lambda^{(a)}=\tau_{j, i, k}(\lambda)^{(a)}$ for all $a \notin\left\{j+l_{k-1} d, j+l_{k} d\right\}$,

- the 2-partitions $\left(\lambda^{\left(j+l_{k-1} d\right)}, \lambda^{\left(j+l_{k} d\right)}\right)$ and $\left(\tau_{j, i, k}(\lambda)^{\left(j+l_{k-1} d\right)}, \tau_{j, i, k}(\lambda)^{\left(j+l_{k} d\right)}\right)=$ $\left(\lambda^{\left(j+l_{k} d\right)}, \lambda^{\left(j+l_{k-1} d\right)}\right)$ have the same ordinary content.

By Proposition 5.3.16, the characters $\chi_{\lambda}$ and $\chi_{\tau_{j, i, k}(\lambda)}$ belong to the same Rouquier block associated with the essential hyperplane $M_{j+l_{k-1} d}=M_{j+l_{k} d}$ and thus, to the same Rouquier block of $\mathcal{H}$. We have

$$
\sigma_{j, i}=\tau_{j, i, 1} \circ \tau_{j, i, 2} \circ \ldots \circ \tau_{j, i, m-1} \circ \tau_{j, i, m} \circ \tau_{j, i, m-1} \circ \ldots \circ \tau_{j, i, 2} \circ \tau_{j, i, 1} .
$$

Consequently, the characters $\chi_{\lambda}$ and $\chi_{\sigma_{j, i}(\lambda)}$ belong to the same Rouquier block of $\mathcal{H}$ for all $j(0 \leq j<d)$ and $i(0 \leq i<e)$.

Thanks to the above result, Proposition 5.5.1 now reads:

Corollary 5.5.3 The block-idempotents of $\left(Z \mathcal{R}_{K}(q) \overline{\mathcal{H}}\right)^{G}$ coincide with the block-idempotents of $\mathcal{R}_{K}(q) \mathcal{H}$.

The following theorem demonstrates how we obtain the Rouquier blocks of $\overline{\mathcal{H}}$ from the Rouquier blocks of $\mathcal{H}$ (already determined in section 5.3).

Theorem 5.5.4 Let $\lambda$ be a de-partition of $r$ and $\chi_{\lambda}$ the corresponding irreducible character of $G($ de, $1, r)$. We define $\operatorname{Irr}(K(q) \overline{\mathcal{H}})_{\lambda}$ to be the subset of $\operatorname{Irr}(K(q) \overline{\mathcal{H}})$ with the property:

$$
\operatorname{Res}_{K(q) \overline{\mathcal{H}}}^{K(q) \mathcal{H}}\left(\chi_{\lambda}\right)=\sum_{\bar{\chi} \in \operatorname{Irr}(K(q) \overline{\mathcal{H}})_{\lambda}} \bar{\chi} .
$$

Then

1. If $\lambda$ is d-stuttering and $\chi_{\lambda}$ is a block of $\mathcal{R}_{K}(q) \mathcal{H}$ by itself, then there

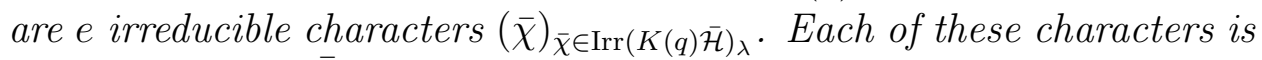
a block of $\mathcal{R}_{K}(q) \overline{\mathcal{H}}$ by itself. 
2. The other blocks of $\mathcal{R}_{K}(q) \mathcal{H}$ are in bijection with the remaining blocks of $\mathcal{R}_{K}(q) \overline{\mathcal{H}}$ via the map of Proposition 2.3.18, i.e., the corresponding block-idempotents of $\mathcal{R}_{K}(q) \mathcal{H}$ coincide with the remaining blockidempotents of $\mathcal{R}_{K}(q) \overline{\mathcal{H}}$.

Proof: If $\lambda$ is a $d$-stuttering partition, then it is the only element in its orbit $\Omega$ under the action of $G^{\vee}$. Set $\bar{\Omega}:=\operatorname{Irr}(K(q) \overline{\mathcal{H}})_{\lambda}$. By Proposition 2.3.15, we have $|\Omega||\bar{\Omega}|=|G|=e$, whence $|\bar{\Omega}|=e$. Moreover, if $\bar{\chi} \in \bar{\Omega}$, then its Schur element $s_{\bar{\chi}}$ is equal to the Schur element $s_{\lambda}$ of $\chi_{\lambda}$. If $\chi_{\lambda}$ is a block of $\mathcal{R}_{K}(q) \mathcal{H}$ by itself, then, Propositions 4.4 .4 and 2.4 .18 imply that $s_{\lambda}=s_{\bar{\chi}}$ is invertible in $\mathcal{R}_{K}(q)$. Thus, $\bar{\chi}$ is a block of $\mathcal{R}_{K}(q) \overline{\mathcal{H}}$ by itself.

If $\lambda$ is $d$-stuttering and $\chi_{\lambda}$ is not a block of $\mathcal{R}_{K}(q) \mathcal{H}$ by itself, then, due to Theorem 5.1.1, there exists a de-partition $\mu \neq \lambda$ such that $\chi_{\lambda}$ and $\chi_{\mu}$ belong to the same Rouquier block associated with an essential hyperplane $H$ for $G(d e, 1, r)$ such that the integers $\left\{\left(m_{a}\right)_{0 \leq a<d e}, e n\right\}$ belong to $H$. If $H$ is $N=0$, then, by Proposition 5.3.15, we have $\left|\lambda^{(a)}\right|=\left|\mu^{(a)}\right|$ for all $a=0,1, \ldots, d e-1$. Since $\lambda \neq \mu$, there exists $b \in\{0,1, \ldots, d e-1\}$ such that $\lambda^{(b)} \neq \mu^{(b)}$. If $\nu$ is the $d e$-partition of $r$ obtained from $\lambda$ by replacing $\lambda^{(b)}$ with $\mu^{(b)}$, then $\chi_{\lambda}$ and $\chi_{\nu}$ belong to the same block of $\mathcal{R}_{K}(q) \mathcal{H}$ and $\nu$ is not $d$-stuttering. If $H$ is of the form $k N+M_{s}-M_{t}=0$, where $-r<k<$ $r$ and $0 \leq s<t<d e$, then $\lambda^{(a)}=\mu^{(a)}$ for all $a \notin\{s, t\}$. If $s \not \equiv t \bmod d$ or $e>2$, then $\mu$ can not be $d$-stuttering. Suppose now that $s \equiv t \bmod d$ and $e=2$. The description of $s_{\lambda}$ by Theorem 6.7.2 implies that the hyperplane $M_{s}=M_{t}$ is not essential for $\chi_{\lambda}$. Due to Proposition 3.2.5, we deduce that $k \neq 0$. Since the integers $\left\{\left(m_{a}\right)_{0 \leq a<d e}\right.$, en $\}$ belong to $H$ and $m_{s}=m_{t}$, we must have $n=0$. If $\mu$ is $d$-stuttering, then $\mu^{(s)}=\mu^{(t)}$ and $\left|\mu^{(s)}\right|=\left|\mu^{(t)}\right|=$ $\left|\lambda^{(t)}\right|=\left|\lambda^{(s)}\right|$. Let $\nu$ be the de-partition obtained from $\lambda$ by replacing $\lambda^{(t)}$ with $\mu^{(t)}$. Then $\nu$ is not $d$-stuttering and the characters $\chi_{\lambda}$ and $\chi_{\nu}$ belong to the same Rouquier block associated with the essential hyperplane $N=0$. Since $n=0$, Theorem 5.1.1 implies that $\chi_{\lambda}$ and $\chi_{\nu}$ belong to the same block of $\mathcal{R}_{K}(q) \mathcal{H}$. We will now show that the blocks of $\mathcal{R}_{K}(q) \mathcal{H}$ which contain at least one character corresponding to a not $d$-stuttering partition are in bijection with the remaining blocks of $\mathcal{R}_{K}(q) \overline{\mathcal{H}}$ via the map of Proposition 2.3 .18 .

Suppose that $\lambda$ is not a $d$-stuttering partition and $b$ is the block containing $\chi_{\lambda}$. Let $\bar{\chi} \in \operatorname{Irr}(K(q) \overline{\mathcal{H}})_{\lambda}$ and let $\bar{b}$ be the block of $\mathcal{R}_{K}(q) \overline{\mathcal{H}}$ which contains $\bar{\chi}$. In order to establish the desired bijection, we have to show that $\bar{b}$ is stable under the action of $G$, i.e., that $\bar{b}=\operatorname{Tr}(G, \bar{b}):=\sum_{g \in G / G_{\bar{b}}} g(\bar{b})$. By Proposition 2.3.15, we have that $b=\operatorname{Tr}(G, \bar{b})$. 
If $\lambda=\left(\lambda^{(0)}, \ldots, \lambda^{(d-1)}, \lambda^{(d)}, \ldots, \lambda^{(2 d-1)}, \ldots, \lambda^{(e d-d)}, \ldots, \lambda^{(e d-1)}\right)$, then, for $i=0,1, \ldots, e-1$, we define the $d$-partition $\lambda_{(i)}$ as follows:

$$
\lambda_{(i)}:=\left(\lambda^{(i d)}, \lambda^{(i d+1)}, \ldots, \lambda^{(i d+d-1)}\right)
$$

and we have

$$
\lambda=\left(\lambda_{(0)}, \lambda_{(1)}, \ldots, \lambda_{(e-1)}\right) .
$$

Since $\lambda$ is not $d$-stuttering, there exists $m \in\{1, \ldots, e-1\}$ such that $\lambda_{(0)} \neq$ $\lambda_{(m)}$. If $p$ is any prime divisor of $e$, we denote by $\lambda(p)$ the de-partition obtained from $\lambda$ by exchanging $\lambda_{(m)}$ and $\lambda_{(e / p)}$. Set

$$
\sigma_{p}:=\prod_{j=0}^{d-1} \sigma_{j, m} \cdot \sigma_{j, e / p} \cdot \sigma_{j, m}
$$

where $\sigma_{j, i}$ denotes the transposition $(j, j+i d)$ for all $i(0 \leq i<e)$. Then $\lambda(p)=\sigma_{p}(\lambda)$. In the proof of Proposition 5.5.2, we showed that the characters $\chi_{\lambda}$ and $\chi_{\sigma_{j, i}(\lambda)}$ are in the same Rouquier block of $\mathcal{H}$ for all $j(0 \leq j<d)$ and $i(0 \leq i<e)$. Therefore, the characters $\chi_{\lambda}$ and $\chi_{\lambda(p)}$ belong to the same block of $\mathcal{R}_{K}(q) \mathcal{H}$. Moreover, by construction, the de-partition $\lambda(p)$ is not fixed by the generator $\tau_{d}^{e / p}$ of the unique subgroup of order $p$ of $G^{\vee}$. Thus, the order of the stabilizer $G_{\chi_{\lambda(p)}}^{\vee}$ of $\chi_{\lambda(p)}$ is prime to $p$.

By Proposition 2.3.15, we know that for each $\bar{\chi}_{p} \in \operatorname{Irr}(K(q) \overline{\mathcal{H}})_{\lambda(p)}$, we have $\left|G_{\chi_{\lambda(p)}}^{\vee}\right|\left|G_{\bar{\chi}_{p}}\right|=e$. Hence, $\left|G_{\bar{\chi}_{p}}\right|$ is divisible by the largest power of $p$ dividing $e$. Since $b=\operatorname{Tr}(G, \bar{b})$, the elements of $\operatorname{Irr}(K(q) \overline{\mathcal{H}})_{\lambda(p)}$ belong to blocks of $\mathcal{R}_{K}(q) \overline{\mathcal{H}}$ conjugate of $\bar{b}$ by $G$, whose stabilizer is $G_{\bar{b}}$. Following Lemma 2.3.16, we deduce that, for any prime number $p,\left|G_{\bar{b}}\right|$ is divisible by the largest power of $p$ dividing $e$. Thus, $G_{\bar{b}}=G$ and $\operatorname{Tr}(G, \bar{b})=\bar{b}$.

Example 5.5.5 Let $d:=1, e:=3$ and $r:=3$. The Hecke algebra of $G(3,3,3)$ is the algebra $\mathcal{H}_{3,3,3}$ generated over the Laurent polynomial ring $\mathbb{Z}\left[x, x^{-1}\right]$ by the elements $a_{1}, a_{2}, a_{3}$ satisfying the relations

- $a_{1} a_{2} a_{1}=a_{2} a_{1} a_{2}, a_{1} a_{3} a_{1}=a_{3} a_{1} a_{3}, a_{2} a_{3} a_{2}=a_{3} a_{2} a_{3}$,

- $a_{1} a_{2} a_{3} a_{1} a_{2} a_{3}=a_{3} a_{1} a_{2} a_{3} a_{1} a_{2}$,

- $\left(a_{1}-x\right)\left(a_{1}+1\right)=\left(a_{2}-x\right)\left(a_{2}+1\right)=\left(a_{3}-x\right)\left(a_{3}+1\right)=0$.

Let $\phi: x \mapsto q^{n}$ with $n \neq 0$ be a cyclotomic specialization of $\mathcal{H}_{3,3,3}$. We can apply Theorem 5.5.4 and obtain the Rouquier blocks of $\left(\mathcal{H}_{3,3,3}\right)_{\phi}$ from the Rouquier 
blocks of $\left(\mathcal{H}_{3,3}\right)_{\vartheta}$, where $\mathcal{H}_{3,3}$ is the generic Ariki-Koike algebra associated to $G(3,1,3)$ and

$$
\vartheta:\left\{\begin{array}{l}
u_{a} \mapsto \zeta_{3}^{a}(0 \leq a \leq 2) \\
x \mapsto q^{n}
\end{array}\right.
$$

Since $n \neq 0$, the Rouquier blocks of $\left(\mathcal{H}_{3,3}\right)_{\vartheta}$ coincide with the Rouquier blocks of $\left(\mathcal{H}_{3,3}\right)_{\theta}$, where

$$
\theta:\left\{\begin{array}{l}
u_{a} \mapsto \zeta_{3}^{a}(0 \leq a \leq 2) \\
x \mapsto q
\end{array}\right.
$$

The latter have been calculated in Example 5.3 .18 and are:

1. $\left\{\chi_{((1),(1),(1))}\right\}$,

2. $\left\{\chi_{((1,1,1), \emptyset, \emptyset)}, \chi_{(\emptyset,(1,1,1), \emptyset)}, \chi_{(\emptyset, \emptyset,(1,1,1))}\right\}$,

3. $\left\{\chi_{((2,1), \emptyset, \emptyset)}, \chi_{(\emptyset,(2,1), \emptyset)}, \chi_{(\emptyset, \emptyset,(2,1))}\right\}$,

4. $\left\{\chi_{((3), \emptyset, \emptyset)}, \chi_{(\emptyset,(3), \emptyset)}, \chi_{(\emptyset, \emptyset,(3))}\right\}$,

5. $\left\{\chi_{((1,1),(1), \emptyset)}, \chi_{((1),(1,1), \emptyset)}, \chi_{((1,1), \emptyset,(1))}, \chi_{((1), \emptyset,(1,1))}, \chi_{(\emptyset,(1,1),(1))}, \chi_{(\emptyset,(1),(1,1))}\right\}$,

6. $\left\{\chi_{((2),(1), \emptyset)}, \chi_{((1),(2), \emptyset)}, \chi_{((2), \emptyset,(1))}, \chi_{((1), \emptyset,(2))}, \chi_{(\emptyset,(2),(1))}, \chi_{(\emptyset,(1),(2))}\right\}$.

Set $\mathcal{H}:=\left(\mathcal{H}_{3,3}\right)_{\vartheta}, \overline{\mathcal{H}}:=\left(\mathcal{H}_{3,3,3}\right)_{\phi}$ and $K:=\mathbb{Q}\left(\zeta_{3}\right)$. We have that

$$
\operatorname{Irr}(K(q) \overline{\mathcal{H}})=\left\{\psi_{1}, \psi_{2}, \ldots, \psi_{10}\right\},
$$

where

- $\psi_{1}=\operatorname{Res}_{K(q) \mathcal{H}^{\prime}}^{K(q) \mathcal{H}}\left(\chi_{((1,1,1), \emptyset, \emptyset)}\right)=\operatorname{Res}_{K(q) \mathcal{\mathcal { H }}}^{K(q) \mathcal{H}}\left(\chi_{(\emptyset,(1,1,1), \emptyset)}\right)=\operatorname{Res}_{K(q) \mathcal{\mathcal { H }}}^{K(q) \mathcal{H}}\left(\chi_{(\emptyset, \emptyset,(1,1,1))}\right)$,

- $\psi_{2}=\operatorname{Res}_{K(q) \mathcal{\mathcal { H }}}^{K(q) \mathcal{H}}\left(\chi_{((2,1), \emptyset, \emptyset)}\right)=\operatorname{Res}_{K(q) \overline{\mathcal{H}}}^{K(q) \mathcal{H}}\left(\chi_{(\emptyset,(2,1), \emptyset)}\right)=\operatorname{Res}_{K(q) \overline{\mathcal{H}}}^{K(q) \mathcal{H}}\left(\chi_{(\emptyset, \emptyset,(2,1))}\right)$,

- $\psi_{3}=\operatorname{Res}_{K(q) \overline{\mathcal{H}}}^{K(q) \mathcal{H}}\left(\chi_{((3), \emptyset, \emptyset)}\right)=\operatorname{Res}_{K(q) \overline{\mathcal{H}}}^{K(q) \mathcal{H}}\left(\chi_{(\emptyset,(3), \emptyset)}\right)=\operatorname{Res}_{K(q) \overline{\mathcal{H}}}^{K\left(\chi^{\prime} \mathcal{H}\right.}\left(\chi_{(\emptyset, \emptyset,(3))}\right)$,

- $\psi_{4}=\operatorname{Res}_{K(q) \mathcal{\mathcal { H }}}^{K(q) \mathcal{H}}\left(\chi_{((1,1),(1), \emptyset)}\right)=\operatorname{Res}_{K(q) \overline{\mathcal{H}}}^{K(q) \mathcal{H}}\left(\chi_{(\emptyset,(1,1),(1))}\right)=\operatorname{Res}_{K(q) \mathcal{\mathcal { H }}}^{K(q) \mathcal{H}}\left(\chi_{((1), \emptyset,(1,1))}\right)$,

- $\psi_{5}=\operatorname{Res}_{K(q) \mathcal{H}^{\prime}}^{K(q) \mathcal{H}}\left(\chi_{((1),(1,1), \emptyset)}\right)=\operatorname{Res}_{K(q) \mathcal{\mathcal { H }}}^{K(q) \mathcal{H}}\left(\chi_{(\emptyset,(1),(1,1))}\right)=\operatorname{Res}_{K(q) \mathcal{\mathcal { H }}}^{K(q) \mathcal{H}}\left(\chi_{((1,1), \emptyset,(1))}\right)$,

- $\psi_{6}=\operatorname{Res}_{K(q) \mathcal{\mathcal { H }}}^{K(q) \mathcal{H}}\left(\chi_{((2),(1), \emptyset)}\right)=\operatorname{Res}_{K(q) \overline{\mathcal{H}}}^{K(q) \mathcal{H}}\left(\chi_{(\emptyset,(2),(1))}\right)=\operatorname{Res}_{K(q) \overline{\mathcal{H}}}^{K(q) \mathcal{H}}\left(\chi_{((1), \emptyset,(2))}\right)$,

- $\psi_{7}=\operatorname{Res}_{K(q) \mathcal{\mathcal { H }}}^{K(q) \mathcal{H}}\left(\chi_{((1),(2), \emptyset)}\right)=\operatorname{Res}_{K(q) \overline{\mathcal{H}}}^{K(q) \mathcal{H}}\left(\chi_{(\emptyset,(1),(2))}\right)=\operatorname{Res}_{K(q) \overline{\mathcal{H}}}^{K(q) \mathcal{H}}\left(\chi_{((2), \emptyset,(1))}\right)$,

- $\psi_{8}+\psi_{9}+\psi_{10}=\operatorname{Res}_{K(q) \overline{\mathcal{H}}}^{K(q) \mathcal{H}}\left(\chi_{((1),(1),(1))}\right)$.

Following Theorem 5.5.4, the Rouquier blocks of $\overline{\mathcal{H}}$ are

$\left\{\psi_{1}\right\},\left\{\psi_{2}\right\},\left\{\psi_{3}\right\},\left\{\psi_{4}, \psi_{5}\right\},\left\{\psi_{6}, \psi_{7}\right\},\left\{\psi_{8}\right\},\left\{\psi_{9}\right\},\left\{\psi_{10}\right\}$. 


\subsubsection{The groups $G(d e, e, 2)$}

If the integer $e$ is odd, then everything that we said in the previous section applies to the case of $G(d e, e, 2)$. Hence, we can obtain the Rouquier blocks of the cyclotomic Hecke algebras of $G(d e, e, 2)$ from those of $G(d e, 1,2)$.

If $e$ is even, then Clifford theory allows us to obtain the Rouquier blocks of the cyclotomic Hecke algebras of $G(d e, e, 2)$ from those of $G(d e, 2,2)$.

Let $f, d \geq 1$. We denote by $\mathcal{H}_{2 f d, 2 f, 2}$ the generic Hecke algebra of $G(2 f d, 2 f, 2)$ generated over the Laurent polynomial ring in $d+4$ indeterminates

$$
\mathbb{Z}\left[x_{0}, x_{0}^{-1}, x_{1}, x_{1}^{-1}, y_{0}, y_{0}^{-1}, y_{1}, y_{1}^{-1}, u_{0}, u_{0}^{-1}, u_{1}, u_{1}^{-1} \ldots, u_{d-1}, u_{d-1}^{-1}\right],
$$

by the elements $S, T, U$ satisfying the relations

- $S T U=U S T, T U S(T S)^{f-1}=U(S T)^{f}$,

- $\left(S-x_{0}\right)\left(S-x_{1}\right)=\left(T-y_{0}\right)\left(T-y_{1}\right)=\left(U-u_{0}\right)\left(U-u_{1}\right) \ldots\left(U-u_{d-1}\right)=0$.

Let

$$
\phi: \begin{cases}x_{i} \mapsto(-1)^{i} q^{a_{i}} & (0 \leq i \leq 1), \\ y_{j} \mapsto(-1)^{j} q^{b_{j}} & (0 \leq j \leq 1), \\ u_{h} \mapsto \zeta_{d}^{h} q^{e_{h}} & (0 \leq h<d) .\end{cases}
$$

be a cyclotomic specialization of $\mathcal{H}_{2 f d, 2 f, 2}$. Following Theorem 5.1.1, the Rouquier blocks of $\left(\mathcal{H}_{2 f d, 2 f, 2}\right)_{\phi}$ coincide with the Rouquier blocks of $\left(\mathcal{H}_{2 f d, 2 f, 2}\right)_{\phi^{f}}$, where

$$
\phi^{f}: \begin{cases}x_{i} \mapsto(-1)^{i} q^{f a_{i}} & (0 \leq i \leq 1), \\ y_{j} \mapsto(-1)^{j} q^{f b_{j}} & (0 \leq j \leq 1), \\ u_{h} \mapsto \zeta_{d}^{h} q^{f e_{h}} & (0 \leq h<d),\end{cases}
$$

since the integers $\left\{a_{i}, b_{j}, e_{h}\right\}$ and $\left\{f a_{i}, f b_{j}, f e_{h}\right\}$ belong to the same essential hyperplanes for $G(2 f d, 2 f, 2)$.

We now consider the generic Hecke algebra $\mathcal{H}_{2 f d}$ of $G(2 f d, 2,2)$ generated over the ring

$$
\mathbb{Z}\left[x_{0}, x_{0}^{-1}, x_{1}, x_{1}^{-1}, y_{0}, y_{0}^{-1}, y_{1}, y_{1}^{-1}, z_{0}, z_{0}^{-1}, z_{1}, z_{1}^{-1} \ldots, z_{f d-1}, z_{f d-1}^{-1}\right]
$$

by the elements $\mathbf{s}, \mathbf{t}, \mathbf{u}$ satisfying the relations

- $\mathbf{s t u}=$ tus $=$ ust,

- $\left(\mathbf{s}-x_{0}\right)\left(\mathbf{s}-x_{1}\right)=\left(\mathbf{t}-y_{0}\right)\left(\mathbf{t}-y_{1}\right)=\left(\mathbf{u}-z_{0}\right)\left(\mathbf{u}-z_{1}\right) \ldots\left(\mathbf{u}-z_{f d-1}\right)=0$.

Let us consider the following cyclotomic specialization of $\mathcal{H}_{2 f d}$ :

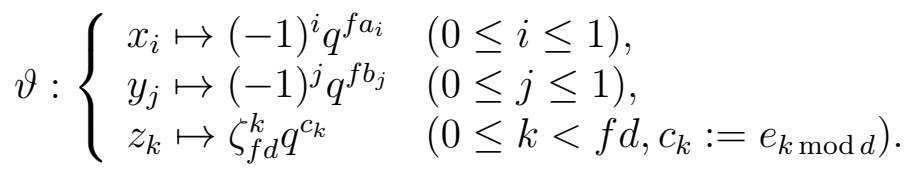


Following Lemma 6.7.3, the algebra $\left(\mathcal{H}_{2 f d}\right)_{\vartheta}$ is the twisted symmetric algebra of the cyclic group $C_{f}$ over the symmetric subalgebra $\left(\mathcal{H}_{2 f d, 2 f, 2}\right)_{\phi^{f}}$.

From now on, set $\mathcal{H}:=\left(\mathcal{H}_{2 f d}\right)_{\vartheta}, \overline{\mathcal{H}}:=\left(\mathcal{H}_{2 f d, 2 f, 2}\right)_{\phi^{f}}, G:=C_{f}, K:=$ $\mathbb{Q}\left(\zeta_{2 f d}\right), y^{|\mu(K)|}:=q$ and let $\mathcal{R}_{K}(y)$ be the Rouquier ring of $K$. Applying Proposition 2.3 .18 gives:

Proposition 5.5.6 The block-idempotents of $\left(Z \mathcal{R}_{K}(y) \overline{\mathcal{H}}\right)^{G}$ coincide with the block-idempotents of $\left(Z \mathcal{R}_{K}(y) \mathcal{H}\right)^{G^{\vee}}$.

The action of the cyclic group $G^{\vee}$ of order $f$ on $\operatorname{Irr}(K(y) \mathcal{H})$ corresponds to the action

$$
\begin{gathered}
\chi_{i, j, k} \mapsto \chi_{i, j, k+d}(0 \leq i, j \leq 1,0 \leq k<f d), \\
\chi_{k, l}^{1,2} \mapsto \chi_{k+d, l+d}^{1,2}(0 \leq k<l<f d),
\end{gathered}
$$

where all the indexes are considered $\bmod f d$.

Let $\chi \in \operatorname{Irr}(K(y) \mathcal{H})$. If we denote by $\Omega$ the orbit of $\chi$ under the action of $G^{\vee}$, then $|\Omega|=f$. We define $\bar{\Omega}$ to be the subset of $\operatorname{Irr}(K(y) \overline{\mathcal{H}})$ with the property:

$$
\operatorname{Res}_{K(y) \overline{\mathcal{H}}}^{K(y) \mathcal{H}}(\chi)=\sum_{\bar{\chi} \in \bar{\Omega}} \bar{\chi}
$$

By Proposition 2.3.15, we know that $|\Omega||\bar{\Omega}|=f$, whence $|\bar{\Omega}|=1$. Since $\bar{\Omega}$ is also the orbit of $\bar{\chi}$ under the action of $G$, we deduce that the blockidempotents of $\mathcal{R}_{K}(y) \overline{\mathcal{H}}$ are fixed by the action of $G$.

With the help of the following lemma, we will show that the Rouquier blocks of $\mathcal{H}$ are also stable under the action of $G^{\vee}$. Here the results of Theorem 5.4.2 are going to be used as definitions.

Lemma 5.5.7 Let $k_{1}, k_{2}$ and $k_{3}$ be three distinct elements of $\{0,1, \ldots, f d-$ $1\}$. If the blocks of $\mathcal{R}_{K}(y) \mathcal{H}$ are unions of the Rouquier blocks associated with the (not necessarily essential) hyperplanes $C_{k_{1}}=C_{k_{2}}$ and $C_{k_{2}}=C_{k_{3}}$, then they are also unions of the Rouquier blocks associated with the (not necessarily essential) hyperplane $C_{k_{1}}=C_{k_{3}}$.

Proof: We only need to show that

(a) the characters $\chi_{i, j, k_{1}}$ and $\chi_{i, j, k_{3}}$ are in the same block of $\mathcal{R}_{K}(y) \mathcal{H}$ for all $0 \leq i, j \leq 1$

(b) the characters $\chi_{k_{1}, m}^{1,2}$ and $\chi_{k_{3}, m}^{1,2}$ are in the same block of $\mathcal{R}_{K}(y) \mathcal{H}$ for all $0 \leq m<f d$ with $m \notin\left\{k_{1}, k_{3}\right\}$. 
Since the blocks of $\mathcal{R}_{K}(y) \mathcal{H}$ are unions of the Rouquier blocks associated with the hyperplanes $C_{k_{1}}=C_{k_{2}}$ and $C_{k_{2}}=C_{k_{3}}$, Theorem 5.4.2 implies that

(1) the characters $\chi_{i, j, k_{1}}$ and $\chi_{i, j, k_{2}}$ are in the same block of $\mathcal{R}_{K}(y) \mathcal{H}$ for all $0 \leq i, j \leq 1$,

(2) the characters $\chi_{i, j, k_{2}}$ and $\chi_{i, j, k_{3}}$ are in the same block of $\mathcal{R}_{K}(y) \mathcal{H}$ for all $0 \leq i, j \leq 1$,

(3) the characters $\chi_{k_{1}, m}^{1,2}$ and $\chi_{k_{2}, m}^{1,2}$ are in the same block of $\mathcal{R}_{K}(y) \mathcal{H}$ for all $0 \leq m<f d$ with $m \notin\left\{k_{1}, k_{2}\right\}$,

(4) the characters $\chi_{k_{2}, m}^{1,2}$ and $\chi_{k_{3}, m}^{1,2}$ are in the same block of $\mathcal{R}_{K}(y) \mathcal{H}$ for all $0 \leq m<f d$ with $m \notin\left\{k_{2}, k_{3}\right\}$.

We immediately deduce (a) for all $0 \leq i, j \leq 1$ and (b) for all $0 \leq m<f d$ with $m \notin\left\{k_{1}, k_{2}, k_{3}\right\}$. Finally, (3) implies that the characters $\chi_{k_{1}, k_{3}}^{1,2}$ and $\chi_{k_{2}, k_{3}}^{1,2}$ are in the same block of $\mathcal{R}_{K}(y) \mathcal{H}$, whereas by (4), $\chi_{k_{1}, k_{2}}^{1,2}$ and $\chi_{k_{1}, k_{3}}^{1,2}$ are also in the same block of $\mathcal{R}_{K}(y) \mathcal{H}$. Thus, the characters $\chi_{k_{1}, k_{2}}^{1,2}$ and $\chi_{k_{2}, k_{3}}^{1,2}$ belong to the same Rouquier block of $\mathcal{H}$.

Theorem 5.5.8 The Rouquier blocks of $\mathcal{H}$ are stable under the action of $G^{\vee}$. In particular, the block-idempotents of $\mathcal{R}_{K}(y) \overline{\mathcal{H}}$ coincide with the blockidempotents of $\mathcal{R}_{K}(y) \mathcal{H}$.

Proof: $\quad$ Following Theorem 5.1.1, the Rouquier blocks of $\mathcal{H}$ are unions of the Rouquier blocks associated with all the essential hyperplanes of the form

$$
C_{h+m d}=C_{h+n d}(0 \leq h<d, 0 \leq m<n<f) .
$$

Recall that the hyperplane $C_{h+m d}=C_{h+n d}$ is actually essential for $G(2 f d, 2,2)$ if and only if the element $\zeta_{f d}^{h+m d}-\zeta_{f d}^{h+n d}$ belongs to a prime ideal of $\mathbb{Z}\left[\zeta_{2 f d}\right]$.

Suppose that $f=p_{1}^{t_{1}} p_{2}^{t_{2}} \ldots p_{r}^{t_{r}}$, where the $p_{i}$ are distinct prime numbers. For $s \in\{1,2, \ldots, r\}$, we set $h_{s}:=f / p_{s}^{t_{s}}$. Then $\operatorname{gcd}\left(h_{s}\right)=1$ and by Bezout's theorem, there exist integers $\left(g_{s}\right)_{1 \leq s \leq r}$ such that $\sum_{s=1}^{r} g_{s} h_{s}=1$. The element $1-\zeta_{f}^{g_{s} h_{s}}$ belongs to all the prime ideals of $\mathbb{Z}\left[\zeta_{2 f d}\right]$ lying over the prime number $p_{s}$. Let $h \in\{0,1, \ldots, d-1\}$ and $m \in\{0,1, \ldots, f-2\}$ and set

$$
l_{0}:=m \text { and } l_{s}:=\left(l_{s-1}+g_{s} h_{s}\right) \bmod f, \text { for all } s(1 \leq s \leq r) .
$$

We have that the element $\zeta_{f d}^{h+l_{s-1} d}-\zeta_{f d}^{h+l_{s} d}=\zeta_{f d}^{h+l_{s-1} d}\left(1-\zeta_{f}^{g_{s} h_{s}}\right)$ belongs to all the prime ideals of $\mathbb{Z}\left[\zeta_{2 f d}\right]$ lying over the prime number $p_{s}$. Therefore, the 
hyperplane $C_{h+l_{s-1} d}=C_{h+l_{s} d}$ is essential for $G(2 f d, 2,2)$ for all $s(1 \leq s \leq r)$. Since $l_{0}=m$ and $l_{r}=m+1$, Lemma 5.5.7 implies that the Rouquier blocks of $\mathcal{H}$ are unions of the Rouquier blocks associated with the (not necessarily essential) hyperplane

$$
C_{h+m d}=C_{h+(m+1) d},
$$

following their description by Theorem 5.4.2. Since this holds for all $m(0 \leq$ $m \leq f-2$ ), Lemma 5.5.7 again implies that the Rouquier blocks of $\mathcal{H}$ are unions of the Rouquier blocks associated with all the hyperplanes of the form

$$
C_{h+m d}=C_{h+n d}(0 \leq m<n<f),
$$

for all $h(0 \leq h<d)$. Consequently, we obtain that

- the characters $\left(\chi_{i, j, h+m d}\right)_{0 \leq m<f}$ are in the same block of $\mathcal{R}_{K}(y) \mathcal{H}$ for all $0 \leq i, j \leq 1$ and $0 \leq h<d$,

- the characters $\left(\chi_{h+m d, h+n d}^{1,2}\right)_{0 \leq m<n<f}$ are in the same block of $\mathcal{R}_{K}(y) \mathcal{H}$ for all $0 \leq h<d$,

- the characters $\left(\chi_{h+m d, h^{\prime}+n d}^{1,2}\right)_{0 \leq m, n<f}$ are in the same block of $\mathcal{R}_{K}(y) \mathcal{H}$ for all $0 \leq h<h^{\prime}<d$.

Thus, the blocks of $\mathcal{R}_{K}(y) \mathcal{H}$ are stable under the action of $G^{\vee}$. Now, Proposition 5.5.6 implies that the block-idempotents of $\mathcal{R}_{K}(y) \overline{\mathcal{H}}$ coincide with the block-idempotents of $\mathcal{R}_{K}(y) \mathcal{H}$.

Thanks to the above result, in order to determine the Rouquier blocks of $\overline{\mathcal{H}}$, it suffices to calculate the Rouquier blocks of $\mathcal{H}$ : If $C$ is a Rouquier block of $\mathcal{H}$, then $\left\{\operatorname{Res}_{K(y) \overline{\mathcal{H}}}^{K(y) \mathcal{H}}(\chi) \mid \chi \in C\right\}$ is a Rouquier block of $\overline{\mathcal{H}}$.

Example 5.5.9 Let $f:=2$ and $d:=1$. The group $G(4,4,2)$ is isomorphic to the group $G(2,1,2)$. The generic Hecke algebra $\mathcal{H}_{4,2,2}$ of $G(4,4,2)$ is generated over the Laurent polynomial ring in 4 indeterminates

$$
\mathbb{Z}\left[x_{0}, x_{0}^{-1}, x_{1}, x_{1}^{-1}, y_{0}, y_{0}^{-1}, y_{1}, y_{1}^{-1}\right],
$$

by the elements $S$ and $T$ satisfying the relations

- $\left(S-x_{0}\right)\left(S-x_{1}\right)=\left(T-y_{0}\right)\left(T-y_{1}\right)=0$.

- $S T S T=T S T S$. 
Let

$$
\phi: \begin{cases}x_{i} \mapsto(-1)^{i} q^{a_{i}} & (0 \leq i \leq 1) \\ y_{j} \mapsto(-1)^{j} q^{b_{j}} & (0 \leq j \leq 1)\end{cases}
$$

be a cyclotomic specialization of $\mathcal{H}_{4,2,2}$. Since $G(4,4,2) \simeq G(2,1,2)$, we can use the results on the Ariki-Koike algebras in order to determine the Rouquier blocks of $\left(\mathcal{H}_{4,2,2}\right)_{\phi}$. However, here we will demonstrate how we can apply Theorem 5.5.8 and obtain the Rouquier blocks of $\left(\mathcal{H}_{4,2,2}\right)_{\phi}$ from the Rouquier blocks of $\left(\mathcal{H}_{4}\right)_{\vartheta}$, where $\mathcal{H}_{4}$ is the generic Hecke algebra associated to $G(4,2,2)$ and

$$
\vartheta: \begin{cases}x_{i} \mapsto(-1)^{i} q^{a_{i}} & (0 \leq i \leq 1), \\ y_{j} \mapsto(-1)^{j} q^{b_{j}} & (0 \leq j \leq 1), \\ z_{k} \mapsto(-1)^{k} & (0 \leq k \leq 1) .\end{cases}
$$

Set $\mathcal{H}:=\left(\mathcal{H}_{4}\right)_{\vartheta}, \overline{\mathcal{H}}:=\left(\mathcal{H}_{4,4,2}\right)_{\phi}, K:=\mathbb{Q}(i)$ and $y^{|\mu(K)|}:=q$. We have that

$$
\operatorname{Irr}(K(y) \overline{\mathcal{H}})=\left\{\chi_{((2), \emptyset)}, \chi_{(\emptyset,(2))}, \chi_{((1,1), \emptyset)}, \chi_{(\emptyset,(1,1))}, \chi_{((1),(1))}\right\},
$$

where

- $\chi_{((2), \emptyset)}=\operatorname{Res}_{K(y) \overline{\mathcal{H}}}^{K(y) \mathcal{H}}\left(\chi_{000}\right)=\operatorname{Res}_{K(y) \overline{\mathcal{H}}}^{K(y) \mathcal{H}}\left(\chi_{001}\right)$,

- $\chi_{(\emptyset,(2))}=\operatorname{Res}_{K(y) \mathcal{H}}^{K(y) \mathcal{H}}\left(\chi_{010}\right)=\operatorname{Res}_{K(y) \mathcal{H}}^{K(y) \mathcal{H}}\left(\chi_{011}\right)$,

- $\chi_{((1,1), \emptyset)}=\operatorname{Res}_{K(y) \mathcal{H}}^{K(y) \mathcal{H}}\left(\chi_{100}\right)=\operatorname{Res}_{K(y) \mathcal{H}}^{K(y) \mathcal{H}}\left(\chi_{101}\right)$,

- $\chi_{(\emptyset,(1,1))}=\operatorname{Res}_{K(y) \overline{\mathcal{H}}}^{K(y) \mathcal{H}}\left(\chi_{110}\right)=\operatorname{Res}_{K(y) \overline{\mathcal{H}}}^{K(y) \mathcal{H}}\left(\chi_{111}\right)$,

- $\chi_{((1),(1))}=\operatorname{Res}_{K(y) \overline{\mathcal{H}}}^{K(y) \mathcal{H}}\left(\chi_{01}^{1}\right)=\operatorname{Res}_{K(y) \overline{\mathcal{H}}}^{K(y) \mathcal{H}}\left(\chi_{01}^{2}\right)$.

Following Theorem 5.5.8, the Rouquier blocks associated with no essential hyperplane for $G(4,4,2)$ are trivial (as expected). For $a_{0}=2, a_{1}=4, b_{0}=3$ and $b_{1}=1$, the Rouquier blocks of $\mathcal{H}$ have been calculated in Example 5.4 .3 and are:

$$
\left\{\chi_{000}, \chi_{001}, \chi_{110}, \chi_{111}, \chi_{01}^{1}, \chi_{01}^{2}\right\},\left\{\chi_{010}, \chi_{011}\right\},\left\{\chi_{100}, \chi_{101}\right\} \text {. }
$$

Thanks to Theorem 5.5.8, we deduce that the Rouquier blocks of $\overline{\mathcal{H}}$ are:

$$
\left\{\chi_{((2), \emptyset)}, \chi_{(\emptyset,(1,1))}, \chi_{((1),(1))}\right\},\left\{\chi_{(\emptyset,(2))}\right\},\left\{\chi_{((1,1), \emptyset)}\right\} .
$$

We can verify the above result with the use of Proposition 5.3.16, which yields that two irreducible characters $\left(\chi_{\lambda}\right)_{\phi}$ and $\left(\chi_{\mu}\right)_{\phi}$ are in the same Rouquier block of $\overline{\mathcal{H}}$ if and only if $\operatorname{Contc}_{\lambda}=\operatorname{Contc}_{\mu}$ with respect to the weight system $(0,1)$. 


\section{Chapter 6}

\section{Appendix: Clifford theory and Schur elements for the Hecke algebras of complex reflection groups}

Let $W$ be a complex reflection group and let us denote by $\mathcal{H}(W)$ its generic Hecke algebra. Suppose that the assumptions 4.2 .3 are satisfied. Let $W^{\prime}$ be another complex reflection group such that, for some specialization of the parameters, $\mathcal{H}(W)$ becomes the twisted symmetric algebra of a finite cyclic group $G$ over the symmetric subalgebra $\mathcal{H}\left(W^{\prime}\right)$. Then, if we know the Schur elements and the blocks of $\mathcal{H}(W)$, we can use Propositions 2.3.15 and 2.3.18 in order to calculate the Schur elements and the blocks of $\mathcal{H}\left(W^{\prime}\right)$.

In particular, in all the cases of exceptional irreducible complex reflection groups that will be studied below, if we denote by $\chi^{\prime}$ the (irreducible) restriction to $\mathcal{H}\left(W^{\prime}\right)$ of an irreducible character $\chi \in \operatorname{Irr}(\mathcal{H}(W))$, then the corresponding Schur elements verify

$$
s_{\chi}=\left|W: W^{\prime}\right| s_{\chi^{\prime}} .
$$

Throughout the Appendix, we denote by $\Phi_{n}$ the $n^{\text {th }} \mathbb{Q}$-cyclotomic polynomial, i.e., the minimal polynomial of $\zeta_{n}$ over $\mathbb{Q}$. The notations for the irreducible characters of the exceptional irreducible complex reflection groups are the ones used by the GAP package CHEVIE and are explained in subsection 5.2.3. 


\subsection{The groups $G_{4}, \ldots, G_{7}$}

The following table gives the specializations of the parameters of the generic Hecke algebra $\mathcal{H}\left(G_{7}\right),\left(x_{0}, x_{1} ; y_{0}, y_{1}, y_{2} ; z_{0}, z_{1}, z_{2}\right)$, which give the generic Hecke algebras of the groups $G_{4}, G_{5}$ and $G_{6}$ ([48], Table 4.6).

\begin{tabular}{|c|c|c|c|c|}
\hline Group & Index & $\mathrm{S}$ & $\mathrm{T}$ & $\mathrm{U}$ \\
\hline$G_{7}$ & 1 & $x_{0}, x_{1}$ & $y_{0}, y_{1}, y_{2}$ & $z_{0}, z_{1}, z_{2}$ \\
$G_{5}$ & 2 & $1,-1$ & $y_{0}, y_{1}, y_{2}$ & $z_{0}, z_{1}, z_{2}$ \\
$G_{6}$ & 3 & $x_{0}, x_{1}$ & $1, \zeta_{3}, \zeta_{3}^{2}$ & $z_{0}, z_{1}, z_{2}$ \\
$G_{4}$ & 6 & $1,-1$ & $1, \zeta_{3}, \zeta_{3}^{2}$ & $z_{0}, z_{1}, z_{2}$ \\
\hline
\end{tabular}

Specializations of the parameters for $\mathcal{H}\left(G_{7}\right)$

\section{Lemma 6.1.1}

1. The algebra $\mathcal{H}\left(G_{7}\right)$ specialized via

$$
\left(x_{0}, x_{1} ; y_{0}, y_{1}, y_{2} ; z_{0}, z_{1}, z_{2}\right) \mapsto\left(1,-1 ; y_{0}, y_{1}, y_{2} ; z_{0}, z_{1}, z_{2}\right)
$$

is the twisted symmetric algebra of the cyclic group $C_{2}$ over the symmetric subalgebra $\mathcal{H}\left(G_{5}\right)$ with parameters $\left(y_{0}, y_{1}, y_{2} ; z_{0}, z_{1}, z_{2}\right)$.

2. The algebra $\mathcal{H}\left(G_{7}\right)$ specialized via

$$
\left(x_{0}, x_{1} ; y_{0}, y_{1}, y_{2} ; z_{0}, z_{1}, z_{2}\right) \mapsto\left(x_{0}, x_{1} ; 1, \zeta_{3}, \zeta_{3}^{2} ; z_{0}, z_{1}, z_{2}\right)
$$

is the twisted symmetric algebra of the cyclic group $C_{3}$ over the symmetric subalgebra $\mathcal{H}\left(G_{6}\right)$ with parameters $\left(x_{0}, x_{1} ; z_{0}, z_{1}, z_{2}\right)$.

3. The algebra $\mathcal{H}\left(G_{6}\right)$ specialized via

$$
\left(x_{0}, x_{1} ; z_{0}, z_{1}, z_{2}\right) \mapsto\left(1,-1 ; z_{0}, z_{1}, z_{2}\right)
$$

is the twisted symmetric algebra of the cyclic group $C_{2}$ over the symmetric subalgebra $\mathcal{H}\left(G_{4}\right)$ with parameters $\left(z_{0}, z_{1}, z_{2}\right)$.

Proof: We have

$$
\begin{aligned}
\mathcal{H}\left(G_{7}\right)=<S, T, U \mid & S T U=T U S=U S T, \\
& \left(S-x_{0}\right)\left(S-x_{1}\right)=0, \\
& \left(T-y_{0}\right)\left(T-y_{1}\right)\left(T-y_{2}\right)=0, \\
& \left(U-z_{0}\right)\left(U-z_{1}\right)\left(U-z_{2}\right)=0>.
\end{aligned}
$$


1. Let

$$
\begin{aligned}
A:=<S, T, U \mid & S T U=T U S=U S T, S^{2}=1, \\
& \left(T-y_{0}\right)\left(T-y_{1}\right)\left(T-y_{2}\right)=0, \\
& \left(U-z_{0}\right)\left(U-z_{1}\right)\left(U-z_{2}\right)=0>
\end{aligned}
$$

and

$$
\bar{A}:=<T, U>.
$$

Then

$$
A=\bar{A} \oplus S \bar{A}=\bar{A} \oplus \bar{A} S \text { and } \bar{A} \cong \mathcal{H}\left(G_{5}\right) .
$$

2. Let

$$
\begin{aligned}
A:=<S, T, U \mid & S T U=T U S=U S T, T^{3}=1, \\
& \left(S-x_{0}\right)\left(S-x_{1}\right)=0, \\
& \left(U-z_{0}\right)\left(U-z_{1}\right)\left(U-z_{2}\right)=0>
\end{aligned}
$$

and

$$
\bar{A}:=<S, U>
$$

Then

$$
A=\bigoplus_{i=0}^{2} T^{i} \bar{A}=\bigoplus_{i=0}^{2} \bar{A} T^{i} \text { and } \bar{A} \cong \mathcal{H}\left(G_{6}\right) .
$$

3. Let

$$
\begin{aligned}
A:=<S, U \mid & S U S U S U=U S U S U S, S^{2}=1, \\
& \left(U-z_{0}\right)\left(U-z_{1}\right)\left(U-z_{2}\right)=0>
\end{aligned}
$$

and

$$
\bar{A}:=<U, S U S>.
$$

Then

$$
A=\bar{A} \oplus S \bar{A}=\bar{A} \oplus \bar{A} S \text { and } \bar{A} \cong \mathcal{H}\left(G_{4}\right) .
$$

The Schur elements of all irreducible characters of $\mathcal{H}\left(G_{7}\right)$ are calculated in [48. They are obtained via Galois transformations (permutation of indeterminates, permutation of roots of unity or combination of the two) from the following ones:

$s_{\phi_{1,0}}=\Phi_{1}\left(x_{0} / x_{1}\right) \cdot \Phi_{1}\left(x_{0} y_{0}^{2} z_{0}^{2} / x_{1} y_{1} y_{2} z_{1} z_{2}\right) \cdot \Phi_{1}\left(y_{0} / y_{1}\right) \cdot \Phi_{1}\left(y_{0} / y_{2}\right) \cdot \Phi_{1}\left(z_{0} / z_{1}\right) \cdot \Phi_{1}\left(z_{0} / z_{2}\right) \cdot$ $\Phi_{1}\left(x_{0} y_{0} z_{0} / x_{1} y_{1} z_{1}\right) \cdot \Phi_{1}\left(x_{0} y_{0} z_{0} / x_{1} y_{1} z_{2}\right) \cdot \Phi_{1}\left(x_{0} y_{0} z_{0} / x_{1} y_{2} z_{1}\right) \cdot \Phi_{1}\left(x_{0} y_{0} z_{0} / x_{1} y_{2} z_{2}\right)$

$s_{\phi_{2,9^{\prime}}}=2 y_{2} / y_{0} \Phi_{1}\left(y_{0} / y_{1}\right) \cdot \Phi_{1}\left(y_{2} / y_{0}\right) \cdot \Phi_{1}\left(z_{1} / z_{0}\right) \cdot \Phi_{1}\left(z_{2} / z_{0}\right) \cdot \Phi_{1}\left(r / x_{0} y_{0} z_{0}\right) \cdot \Phi_{1}\left(r / x_{0} y_{2} z_{1}\right) \cdot$ $\Phi_{1}\left(r / x_{0} y_{2} z_{2}\right) \cdot \Phi_{1}\left(r / x_{1} y_{0} z_{0}\right) \cdot \Phi_{1}\left(r / x_{1} y_{2} z_{1}\right) \cdot \Phi_{1}\left(r / x_{1} y_{2} z_{2}\right)$

where $r=\sqrt[2]{x_{0} x_{1} y_{1} y_{2} z_{1} z_{2}}$ 
$s_{\phi_{3,6}}=3 \Phi_{1}\left(x_{1} / x_{0}\right) \cdot \Phi_{1}\left(x_{0} y_{0} z_{0} / r\right) \cdot \Phi_{1}\left(x_{0} y_{0} z_{1} / r\right) \cdot \Phi_{1}\left(x_{0} y_{0} z_{2} / r\right) \cdot \Phi_{1}\left(x_{0} y_{1} z_{0} / r\right) \cdot \Phi_{1}\left(x_{0} y_{1} z_{1} / r\right) \cdot$

$\Phi_{1}\left(x_{0} y_{1} z_{2} / r\right) \cdot \Phi_{1}\left(x_{0} y_{2} z_{0} / r\right) \cdot \Phi_{1}\left(x_{0} y_{2} z_{1} / r\right) \cdot \Phi_{1}\left(x_{0} y_{2} z_{2} / r\right)$

where $r=\sqrt[3]{x_{0}^{2} x_{1} y_{0} y_{1} y_{2} z_{0} z_{1} z_{2}}$

Following Theorem 4.2.4 and [49], Table 8.1, if we set

$$
\begin{array}{ll}
X_{i}^{12}:=\left(\zeta_{2}\right)^{-i} x_{i} & (i=0,1) \\
Y_{j}^{12}:=\left(\zeta_{3}\right)^{-j} y_{j} & (j=0,1,2) \\
Z_{k}^{12}:=\left(\zeta_{3}\right)^{-k} z_{k} & (k=0,1,2)
\end{array}
$$

then $\mathbb{Q}\left(\zeta_{12}\right)\left(X_{0}, X_{1}, Y_{0}, Y_{1}, Y_{2}, Z_{0}, Z_{1}, Z_{2}\right)$ is a splitting field for $\mathcal{H}\left(G_{7}\right)$. Hence, the factorization of the Schur elements over that field is as described by Theorem 4.2.5.

\subsection{The groups $G_{8}, \ldots, G_{15}$}

The following table gives the specializations of the parameters of the generic Hecke algebra $\mathcal{H}\left(G_{11}\right),\left(x_{0}, x_{1} ; y_{0}, y_{1}, y_{2} ; z_{0}, z_{1}, z_{2}, z_{3}\right)$, which give the generic Hecke algebras of the groups $G_{8}, \ldots, G_{15}$ ([48], Table 4.9).

\begin{tabular}{|c|c|c|c|c|}
\hline Group & Index & $\mathrm{S}$ & $\mathrm{T}$ & $\mathrm{U}$ \\
\hline$G_{11}$ & 1 & $x_{0}, x_{1}$ & $y_{0}, y_{1}, y_{2}$ & $z_{0}, z_{1}, z_{2}, z_{3}$ \\
$G_{10}$ & 2 & $1,-1$ & $y_{0}, y_{1}, y_{2}$ & $z_{1}, z_{1}, z_{2}, z_{3}$ \\
$G_{15}$ & 2 & $x_{0}, x_{1}$ & $y_{0}, y_{1}, y_{2}$ & $\sqrt{u_{0}}, \sqrt{u_{1}},-\sqrt{u_{0}},-\sqrt{u_{1}}$ \\
$G_{9}$ & 3 & $x_{0}, x_{1}$ & $1, \zeta_{3}, \zeta_{3}^{2}$ & $z_{0}, z_{1}, z_{2}, z_{3}$ \\
$G_{14}$ & 4 & $x_{0}, x_{1}$ & $y_{0}, y_{1}, y_{2}$ & $1, i,-1,-i$ \\
$G_{8}$ & 6 & $1,-1$ & $1, \zeta_{3}, \zeta_{3}^{2}$ & $z_{0}, z_{1}, z_{2}, z_{3}$ \\
$G_{13}$ & 6 & $x_{0}, x_{1}$ & $1, \zeta_{3}, \zeta_{3}^{2}$ & $\sqrt{u_{0}}, \sqrt{u_{1}},-\sqrt{u_{0}},-\sqrt{u_{1}}$ \\
$G_{12}$ & 12 & $x_{0}, x_{1}$ & $1, \zeta_{3}, \zeta_{3}^{2}$ & $1, i,-1,-i$ \\
\hline
\end{tabular}

Specializations of the parameters for $\mathcal{H}\left(G_{11}\right)$

\section{Lemma 6.2.1}

1. The algebra $\mathcal{H}\left(G_{11}\right)$ specialized via

$$
\left(x_{0}, x_{1} ; y_{0}, y_{1}, y_{2} ; z_{0}, z_{1}, z_{2}, z_{3}\right) \mapsto\left(1,-1 ; y_{0}, y_{1}, y_{2} ; z_{0}, z_{1}, z_{2}, z_{3}\right)
$$

is the twisted symmetric algebra of the cyclic group $C_{2}$ over the symmetric subalgebra $\mathcal{H}\left(G_{10}\right)$ with parameters $\left(y_{0}, y_{1}, y_{2} ; z_{0}, z_{1}, z_{2}, z_{3}\right)$. 
2. The algebra $\mathcal{H}\left(G_{11}\right)$ specialized via

$$
\left(x_{0}, x_{1} ; y_{0}, y_{1}, y_{2} ; z_{0}, z_{1}, z_{2}, z_{3}\right) \mapsto\left(x_{0}, x_{1} ; 1, \zeta_{3}, \zeta_{3}^{2} ; z_{0}, z_{1}, z_{2}, z_{3}\right)
$$

is the twisted symmetric algebra of the cyclic group $C_{3}$ over the symmetric subalgebra $\mathcal{H}\left(G_{9}\right)$ with parameters $\left(x_{0}, x_{1} ; z_{0}, z_{1}, z_{2}, z_{3}\right)$.

3. The algebra $\mathcal{H}\left(G_{9}\right)$ specialized via

$$
\left(x_{0}, x_{1} ; z_{0}, z_{1}, z_{2}, z_{3}\right) \mapsto\left(1,-1 ; z_{0}, z_{1}, z_{2}, z_{3}\right)
$$

is the twisted symmetric algebra of the cyclic group $C_{2}$ over the symmetric subalgebra $\mathcal{H}\left(G_{8}\right)$ with parameters $\left(z_{0}, z_{1}, z_{2}, z_{3}\right)$.

4. The algebra $\mathcal{H}\left(G_{11}\right)$ specialized via

$$
\left(x_{0}, x_{1} ; y_{0}, y_{1}, y_{2} ; z_{0}, z_{1}, z_{2}, z_{3}\right) \mapsto\left(x_{0}, x_{1} ; y_{0}, y_{1}, y_{2} ; 1, i,-1,-i\right)
$$

is the twisted symmetric algebra of the cyclic group $C_{4}$ over the symmetric subalgebra $\mathcal{H}\left(G_{14}\right)$ with parameters $\left(x_{0}, x_{1} ; y_{0}, y_{1}, y_{2}\right)$.

5. The algebra $\mathcal{H}\left(G_{14}\right)$ specialized via

$$
\left(x_{0}, x_{1} ; y_{0}, y_{1}, y_{2}\right) \mapsto\left(x_{0}, x_{1} ; 1, \zeta_{3}, \zeta_{3}^{2}\right)
$$

is the twisted symmetric algebra of the cyclic group $C_{3}$ over the symmetric subalgebra $\mathcal{H}\left(G_{12}\right)$ with parameters $\left(x_{0}, x_{1}\right)$.

6. The algebra $\mathcal{H}\left(G_{11}\right)$ specialized via

$\left(x_{0}, x_{1} ; y_{0}, y_{1}, y_{2} ; z_{0}, z_{1}, z_{2}, z_{3}\right) \mapsto\left(x_{0}, x_{1} ; y_{0}, y_{1}, y_{2} ; \sqrt{u_{0}}, \sqrt{u_{1}},-\sqrt{u_{0}},-\sqrt{u_{1}}\right)$

is the twisted symmetric algebra of the cyclic group $C_{2}$ over the symmetric subalgebra $\mathcal{H}\left(G_{15}\right)$ with parameters $\left(x_{0}, x_{1} ; y_{0}, y_{1}, y_{2} ; u_{0}, u_{1}\right)$.

7. The algebra $\mathcal{H}\left(G_{15}\right)$ specialized via

$$
\left(x_{0}, x_{1} ; y_{0}, y_{1}, y_{2} ; u_{0}, u_{1}\right) \mapsto\left(x_{0}, x_{1} ; 1, \zeta_{3}, \zeta_{3}^{2} ; u_{0}, u_{1}\right)
$$

is the twisted symmetric algebra of the cyclic group $C_{3}$ over the symmetric subalgebra $\mathcal{H}\left(G_{13}\right)$ with parameters $\left(x_{0}, x_{1} ; u_{0}, u_{1}\right)$.

Proof: We have

$$
\begin{aligned}
\mathcal{H}\left(G_{11}\right)=<S, T, U \mid & S T U=T U S=U S T, \\
& \left(S-x_{0}\right)\left(S-x_{1}\right)=0, \\
& \left(T-y_{0}\right)\left(T-y_{1}\right)\left(T-y_{2}\right)=0, \\
& \left(U-z_{0}\right)\left(U-z_{1}\right)\left(U-z_{2}\right)\left(U-z_{3}\right)=0>.
\end{aligned}
$$


1. Let

$$
\begin{aligned}
A:=<S, T, U \mid & S T U=T U S=U S T, S^{2}=1 \\
& \left(T-y_{0}\right)\left(T-y_{1}\right)\left(T-y_{2}\right)=0, \\
& \left(U-z_{0}\right)\left(U-z_{1}\right)\left(U-z_{2}\right)\left(U-z_{3}\right)=0>
\end{aligned}
$$

and

$$
\bar{A}:=<T, U>.
$$

Then

$$
A=\bar{A} \oplus S \bar{A}=\bar{A} \oplus \bar{A} S \text { and } \bar{A} \cong \mathcal{H}\left(G_{10}\right) .
$$

2. Let

$$
\begin{aligned}
A:=<S, T, U \mid & S T U=T U S=U S T, T^{3}=1, \\
& \left(S-x_{0}\right)\left(S-x_{1}\right)=0 \\
& \left(U-z_{0}\right)\left(U-z_{1}\right)\left(U-z_{2}\right)\left(U-z_{3}\right)=0>
\end{aligned}
$$

and

$$
\bar{A}:=<S, U>.
$$

Then

$$
A=\bigoplus_{i=0}^{2} T^{i} \bar{A}=\bigoplus_{i=0}^{2} \bar{A} T^{i} \text { and } \bar{A} \cong \mathcal{H}\left(G_{9}\right) .
$$

3. Let

$$
\begin{aligned}
A:=<S, U \mid & S U S U S U=U S U S U S, S^{2}=1 \\
& \left(U-z_{0}\right)\left(U-z_{1}\right)\left(U-z_{2}\right)\left(U-z_{3}\right)=0>
\end{aligned}
$$

and

$$
\bar{A}:=<U, S U S>\text {. }
$$

Then

$$
A=\bar{A} \oplus S \bar{A}=\bar{A} \oplus \bar{A} S \text { and } \bar{A} \cong \mathcal{H}\left(G_{8}\right) .
$$

4. Let

$$
\begin{aligned}
A:=<S, T, U \mid & S T U=T U S=U S T, U^{4}=1, \\
& \left(S-x_{0}\right)\left(S-x_{1}\right)=0, \\
& \left(T-y_{0}\right)\left(T-y_{1}\right)\left(T-y_{2}\right)=0>
\end{aligned}
$$

and

$$
\bar{A}:=<S, T>.
$$

Then

$$
A=\bigoplus_{i=0}^{3} U^{i} \bar{A}=\bigoplus_{i=0}^{3} \bar{A} U^{i} \text { and } \bar{A} \cong \mathcal{H}\left(G_{14}\right) .
$$


5. Let

$$
\begin{aligned}
A:=<S, T \mid & \text { STSTSTST }=\text { TSTSTSTS }, T^{3}=1 \\
& \left(S-x_{0}\right)\left(S-x_{1}\right)=0>
\end{aligned}
$$

and

$$
\bar{A}:=<S, T S T^{2}, T^{2} S T>.
$$

Then

$$
A=\bigoplus_{i=0}^{2} T^{i} \bar{A}=\bigoplus_{i=0}^{2} \bar{A} T^{i} \text { and } \bar{A} \cong \mathcal{H}\left(G_{12}\right) .
$$

6. Let

$$
\begin{aligned}
A:=<S, T, U \mid & S T U=T U S=U S T, \\
& \left(S-x_{0}\right)\left(S-x_{1}\right)=0, \\
& \left(T-y_{0}\right)\left(T-y_{1}\right)\left(T-y_{2}\right)=0, \\
& \left(U^{2}-u_{0}\right)\left(U^{2}-u_{1}\right)=0>
\end{aligned}
$$

and

$$
\bar{A}:=<S, T, U^{2}>
$$

Then

$$
A=\bar{A} \oplus U \bar{A}=\bar{A} \oplus \bar{A} U \text { and } \bar{A} \cong \mathcal{H}\left(G_{15}\right) .
$$

7. Let

$$
\begin{aligned}
A:=<U^{2}, S, T \mid & S T U^{2}=U^{2} S T, U^{2} S T S T=T U^{2} S T S, T^{3}=1, \\
& \left(S-x_{0}\right)\left(S-x_{1}\right)=0, \\
& \left(U^{2}-u_{0}\right)\left(U^{2}-u_{1}\right)=0>
\end{aligned}
$$

and

$$
\bar{A}:=<U^{2}, S, T^{2} S T>
$$

Then

$$
A=\bigoplus_{i=0}^{2} T^{i} \bar{A}=\bigoplus_{i=0}^{2} \bar{A} T^{i} \text { and } \bar{A} \cong \mathcal{H}\left(G_{13}\right) .
$$

The Schur elements of all irreducible characters of $\mathcal{H}\left(G_{11}\right)$ are calculated in [48]. They are obtained via Galois transformations from the following ones:

$$
\begin{aligned}
& s_{\phi_{1,0}}=\Phi_{1}\left(x_{0} / x_{1}\right) \cdot \Phi_{1}\left(y_{0} / y_{1}\right) \cdot \Phi_{1}\left(y_{0} / y_{2}\right) \cdot \Phi_{1}\left(z_{0} / z_{1}\right) \cdot \Phi_{1}\left(z_{0} / z_{2}\right) \cdot \Phi_{1}\left(z_{0} / z_{3}\right) \cdot \Phi_{1}\left(x_{0} y_{0} z_{0} / x_{1} y_{1} z_{1}\right) \cdot \\
& \Phi_{1}\left(x_{0} y_{0} z_{0} / x_{1} y_{1} z_{2}\right) \cdot \Phi_{1}\left(x_{0} y_{0} z_{0} / x_{1} y_{1} z_{3}\right) \cdot \Phi_{1}\left(x_{0} y_{0} z_{0} / x_{1} y_{2} z_{1}\right) \cdot \Phi_{1}\left(x_{0} y_{0} z_{0} / x_{1} y_{2} z_{2}\right) \cdot \\
& \Phi_{1}\left(x_{0} y_{0} z_{0} / x_{1} y_{2} z_{3}\right) \cdot \Phi_{1}\left(x_{0} y_{0}^{2} z_{0}^{2} / x_{1} y_{1} y_{2} z_{1} z_{2}\right) \cdot \Phi_{1}\left(x_{0} y_{0}^{2} z_{0}^{2} / x_{1} y_{1} y_{2} z_{1} z_{3}\right) \cdot \Phi_{1}\left(x_{0} y_{0}^{2} z_{0}^{2} / x_{1} y_{1} y_{2} z_{2} z_{3}\right) \cdot \\
& \Phi_{1}\left(x_{0}^{2} y_{0}^{2} z_{0}^{3} / x_{1}^{2} y_{1} y_{2} z_{1} z_{2} z_{3}\right) \\
& s_{\phi_{2,1}}=-2 z_{1} / z_{0} \Phi_{1}\left(y_{0} / y_{2}\right) \cdot \Phi_{1}\left(y_{1} / y_{2}\right) \cdot \Phi_{1}\left(z_{0} / z_{2}\right) \cdot \Phi_{1}\left(z_{0} / z_{3}\right) \cdot \Phi_{1}\left(z_{1} / z_{2}\right) \cdot \Phi_{1}\left(z_{1} / z_{3}\right) \cdot
\end{aligned}
$$


$\Phi_{1}\left(y_{0} z_{0} z_{1} / y_{2} z_{2} z_{3}\right) \cdot \Phi_{1}\left(y_{1} z_{0} z_{1} / y_{2} z_{2} z_{3}\right) \cdot \Phi_{1}\left(r / x_{0} y_{2} z_{2}\right) \cdot \Phi_{1}\left(r / x_{0} y_{2} z_{3}\right) \cdot \Phi_{1}\left(r / x_{1} y_{2} z_{2}\right) \cdot$

$\Phi_{1}\left(r / x_{1} y_{2} z_{3}\right) \cdot \Phi_{1}\left(r / x_{0} y_{0} z_{1}\right) \cdot \Phi_{1}\left(r / x_{0} y_{1} z_{1}\right) \cdot \Phi_{1}\left(r / x_{1} y_{0} z_{1}\right) \cdot \Phi_{1}\left(r / x_{1} y_{1} z_{1}\right)$

where $r=\sqrt[2]{x_{0} x_{1} y_{0} y_{1} z_{0} z_{1}}$

$s_{\phi_{3,2}}=3 \Phi_{1}\left(x_{1} / x_{0}\right) \cdot \Phi_{1}\left(z_{1} / z_{3}\right) \cdot \Phi_{1}\left(z_{2} / z_{3}\right) \cdot \Phi_{1}\left(z_{0} / z_{3}\right) \cdot \Phi_{1}\left(r / x_{1} y_{0} z_{3}\right) \cdot \Phi_{1}\left(r / x_{1} y_{1} z_{3}\right) \cdot$

$\Phi_{1}\left(r / x_{1} y_{2} z_{3}\right) \cdot \Phi_{1}\left(x_{0} y_{0} z_{0} / r\right) \cdot \Phi_{1}\left(x_{0} y_{0} z_{1} / r\right) \cdot \Phi_{1}\left(x_{0} y_{0} z_{2} / r\right) \cdot \Phi_{1}\left(x_{0} y_{1} z_{0} / r\right) \cdot$

$\Phi_{1}\left(x_{0} y_{1} z_{1} / r\right) \cdot \Phi_{1}\left(x_{0} y_{1} z_{2} / r\right) \cdot \Phi_{1}\left(x_{0} y_{2} z_{0} / r\right) \cdot \Phi_{1}\left(x_{0} y_{2} z_{1} / r\right) \cdot \Phi_{1}\left(x_{0} y_{2} z_{2} / r\right)$

where $r=\sqrt[3]{x_{0}^{2} x_{1} y_{0} y_{1} y_{2} z_{0} z_{1} z_{2}}$

$s_{\phi_{4,21}}=-4 \Phi_{1}\left(y_{0} / y_{1}\right) \cdot \Phi_{1}\left(y_{0} / y_{2}\right) \cdot \Phi_{1}\left(r / x_{0} y_{0} z_{0}\right) \cdot \Phi_{1}\left(r / x_{1} y_{0} z_{0}\right) \cdot \Phi_{1}\left(x_{0} y_{0} z_{1} / r\right) \cdot \Phi_{1}\left(x_{0} y_{0} z_{2} / r\right) \cdot$

$\Phi_{1}\left(x_{0} y_{0} z_{3} / r\right) \cdot \Phi_{1}\left(x_{1} y_{0} z_{1} / r\right) \cdot \Phi_{1}\left(x_{1} y_{0} z_{2} / r\right) \cdot \Phi_{1}\left(x_{1} y_{0} z_{3} / r\right) \cdot$

$\Phi_{1}\left(x_{0} x_{1} y_{0} y_{1} z_{0} z_{1} / r^{2}\right) \cdot \Phi_{1}\left(x_{0} x_{1} y_{0} y_{1} z_{0} z_{2} / r^{2}\right) \cdot \Phi_{1}\left(x_{0} x_{1} y_{0} y_{1} z_{0} z_{3} / r^{2}\right) \cdot$

$\Phi_{1}\left(x_{0} x_{1} y_{0} y_{2} z_{0} z_{1} / r^{2}\right) \cdot \Phi_{1}\left(x_{0} x_{1} y_{0} y_{2} z_{0} z_{2} / r^{2}\right) \cdot \Phi_{1}\left(x_{0} x_{1} y_{0} y_{2} z_{0} z_{3} / r^{2}\right)$

where $r=\sqrt[4]{x_{0}^{2} x_{1}^{2} y_{0}^{2} y_{1} y_{2} z_{0} z_{1} z_{2} z_{3}}$

Following Theorem 4.2.4 and 49, Table 8.1, if we set

$$
\begin{array}{ll}
X_{i}^{24}:=\left(\zeta_{2}\right)^{-i} x_{i} & (i=0,1), \\
Y_{j}^{24}:=\left(\zeta_{3}\right)^{-j} y_{j} & (j=0,1,2), \\
Z_{k}^{24}:=\left(\zeta_{4}\right)^{-k} z_{k} & (k=0,1,2,3),
\end{array}
$$

then $\mathbb{Q}\left(\zeta_{24}\right)\left(X_{0}, X_{1}, Y_{0}, Y_{1}, Y_{2}, Z_{0}, Z_{1}, Z_{2}, Z_{3}\right)$ is a splitting field for $\mathcal{H}\left(G_{11}\right)$. Hence, the factorization of the Schur elements over that field is as described by Theorem 4.2.5.

\subsection{The groups $G_{16}, \ldots, G_{22}$}

The following table gives the specializations of the parameters of the generic Hecke algebra $\mathcal{H}\left(G_{19}\right),\left(x_{0}, x_{1} ; y_{0}, y_{1}, y_{2} ; z_{0}, z_{1}, z_{2}, z_{3}, z_{4}\right)$, which give the generic Hecke algebras of the groups $G_{16}, \ldots, G_{22}$ ([48], Table 4.12).

\begin{tabular}{|c|c|c|c|c|}
\hline Group & Index & $\mathrm{S}$ & $\mathrm{T}$ & $\mathrm{U}$ \\
\hline$G_{19}$ & 1 & $x_{0}, x_{1}$ & $y_{0}, y_{1}, y_{2}$ & $z_{0}, z_{1}, z_{2}, z_{3}, z_{4}$ \\
$G_{18}$ & 2 & $1,-1$ & $y_{0}, y_{1}, y_{2}$ & $z_{0}, z_{1}, z_{2}, z_{3}, z_{4}$ \\
$G_{17}$ & 3 & $x_{0}, x_{1}$ & $1, \zeta_{3}, \zeta_{3}^{2}$ & $z_{0}, z_{1}, z_{2}, z_{3}, z_{4}$ \\
$G_{21}$ & 5 & $x_{0}, x_{1}$ & $y_{0}, y_{1}, y_{2}$ & $1, \zeta_{5}, \zeta_{5}^{2}, \zeta_{5}^{3}, \zeta_{5}^{4}$ \\
$G_{16}$ & 6 & $1,-1$ & $1, \zeta_{3}, \zeta_{3}^{2}$ & $z_{0}, z_{1}, z_{2}, z_{3}, z_{4}$ \\
$G_{20}$ & 10 & $1,-1$ & $y_{0}, y_{1}, y_{2}$ & $1, \zeta_{5}, \zeta_{5}^{2}, \zeta_{5}^{3}, \zeta_{5}^{4}$ \\
$G_{22}$ & 15 & $x_{0}, x_{1}$ & $1, \zeta_{3}, \zeta_{3}^{2}$ & $1, \zeta_{5}, \zeta_{5}^{2}, \zeta_{5}^{3}, \zeta_{5}^{4}$ \\
\hline
\end{tabular}

Specializations of the parameters for $\mathcal{H}\left(G_{19}\right)$ 


\section{Lemma 6.3.1}

1. The algebra $\mathcal{H}\left(G_{19}\right)$ specialized via

$$
\left(x_{0}, x_{1} ; y_{0}, y_{1}, y_{2} ; z_{0}, z_{1}, z_{2}, z_{3}, z_{4}\right) \mapsto\left(1,-1 ; y_{0}, y_{1}, y_{2} ; z_{0}, z_{1}, z_{2}, z_{3}, z_{4}\right)
$$

is the twisted symmetric algebra of the cyclic group $C_{2}$ over the symmetric subalgebra $\mathcal{H}\left(G_{18}\right)$ with parameters $\left(y_{0}, y_{1}, y_{2} ; z_{0}, z_{1}, z_{2}, z_{3}, z_{4}\right)$.

2. The algebra $\mathcal{H}\left(G_{19}\right)$ specialized via

$$
\left(x_{0}, x_{1} ; y_{0}, y_{1}, y_{2} ; z_{0}, z_{1}, z_{2}, z_{3}, z_{4}\right) \mapsto\left(x_{0}, x_{1} ; 1, \zeta_{3}, \zeta_{3}^{2} ; z_{0}, z_{1}, z_{2}, z_{3}, z_{4}\right)
$$

is the twisted symmetric algebra of the cyclic group $C_{3}$ over the symmetric subalgebra $\mathcal{H}\left(G_{17}\right)$ with parameters $\left(x_{0}, x_{1} ; z_{0}, z_{1}, z_{2}, z_{3}, z_{4}\right)$.

3. The algebra $\mathcal{H}\left(G_{17}\right)$ specialized via

$$
\left(x_{0}, x_{1} ; z_{0}, z_{1}, z_{2}, z_{3}, z_{4}\right) \mapsto\left(1,-1 ; z_{0}, z_{1}, z_{2}, z_{3}, z_{4}\right)
$$

is the twisted symmetric algebra of the cyclic group $C_{2}$ over the symmetric subalgebra $\mathcal{H}\left(G_{16}\right)$ with parameters $\left(z_{0}, z_{1}, z_{2}, z_{3}, z_{4}\right)$.

4. The algebra $\mathcal{H}\left(G_{19}\right)$ specialized via

$$
\left(x_{0}, x_{1} ; y_{0}, y_{1}, y_{2} ; z_{0}, z_{1}, z_{2}, z_{3}, z_{4}\right) \mapsto\left(x_{0}, x_{1} ; y_{0}, y_{1}, y_{2} ; 1, \zeta_{5}, \zeta_{5}^{2}, \zeta_{5}^{3}, \zeta_{5}^{4}\right)
$$

is the twisted symmetric algebra of the cyclic group $C_{5}$ over the symmetric subalgebra $\mathcal{H}\left(G_{21}\right)$ with parameters $\left(x_{0}, x_{1} ; y_{0}, y_{1}, y_{2}\right)$.

5. The algebra $\mathcal{H}\left(G_{21}\right)$ specialized via

$$
\left(x_{0}, x_{1} ; y_{0}, y_{1}, y_{2}\right) \mapsto\left(1,-1 ; y_{0}, y_{1}, y_{2}\right)
$$

is the twisted symmetric algebra of the cyclic group $C_{2}$ over the symmetric subalgebra $\mathcal{H}\left(G_{20}\right)$ with parameters $\left(y_{0}, y_{1}, y_{2}\right)$.

6. The algebra $\mathcal{H}\left(G_{21}\right)$ specialized via

$$
\left(x_{0}, x_{1} ; y_{0}, y_{1}, y_{2}\right) \mapsto\left(x_{0}, x_{1} ; 1, \zeta_{3}, \zeta_{3}^{2}\right)
$$

is the twisted symmetric algebra of the cyclic group $C_{3}$ over the symmetric subalgebra $\mathcal{H}\left(G_{22}\right)$ with parameters $\left(x_{0}, x_{1}\right)$. 
Proof: We have

$$
\begin{aligned}
\mathcal{H}\left(G_{19}\right)=<S, T, U \mid & S T U=T U S=U S T, \\
& \left(S-x_{0}\right)\left(S-x_{1}\right)=0, \\
& \left(T-y_{0}\right)\left(T-y_{1}\right)\left(T-y_{2}\right)=0, \\
& \left(U-z_{0}\right)\left(U-z_{1}\right)\left(U-z_{2}\right)\left(U-z_{3}\right)\left(U-z_{4}\right)=0>.
\end{aligned}
$$

1. Let

$$
\begin{aligned}
A:=<S, T, U \mid & S T U=T U S=U S T, S^{2}=1, \\
& \left(T-y_{0}\right)\left(T-y_{1}\right)\left(T-y_{2}\right)=0, \\
& \left(U-z_{0}\right)\left(U-z_{1}\right)\left(U-z_{2}\right)\left(U-z_{3}\right)\left(U-z_{4}\right)=0>
\end{aligned}
$$

and

$$
\bar{A}:=<T, U>.
$$

Then

$$
A=\bar{A} \oplus S \bar{A}=\bar{A} \oplus \bar{A} S \text { and } \bar{A} \cong \mathcal{H}\left(G_{18}\right) .
$$

2. Let

$$
\begin{aligned}
A:=<S, T, U \mid & S T U=T U S=U S T, T^{3}=1 \\
& \left(S-x_{0}\right)\left(S-x_{1}\right)=0 \\
& \left(U-z_{0}\right)\left(U-z_{1}\right)\left(U-z_{2}\right)\left(U-z_{3}\right)\left(U-z_{4}\right)=0>
\end{aligned}
$$

and

$$
\bar{A}:=<S, U>.
$$

Then

$$
A=\bigoplus_{i=0}^{2} T^{i} \bar{A}=\bigoplus_{i=0}^{2} \bar{A} T^{i} \text { and } \bar{A} \cong \mathcal{H}\left(G_{17}\right)
$$

3. Let

$$
\begin{aligned}
A:=<S, U \mid & S U S U S U=U S U S U S, S^{2}=1 \\
& \left(U-z_{0}\right)\left(U-z_{1}\right)\left(U-z_{2}\right)\left(U-z_{3}\right)\left(U-z_{4}\right)=0>
\end{aligned}
$$

and

$$
\bar{A}:=<U, S U S>.
$$

Then

$$
A=\bar{A} \oplus S \bar{A}=\bar{A} \oplus \bar{A} S \text { and } \bar{A} \cong \mathcal{H}\left(G_{16}\right) .
$$

4. Let

$$
\begin{aligned}
A:=<S, T, U \mid & S T U=T U S=U S T, U^{5}=1, \\
& \left(S-x_{0}\right)\left(S-x_{1}\right)=0, \\
& \left(T-y_{0}\right)\left(T-y_{1}\right)\left(T-y_{2}\right)=0>
\end{aligned}
$$

and

$$
\bar{A}:=<S, T>.
$$


Then

$$
A=\bigoplus_{i=0}^{4} U^{i} \bar{A}=\bigoplus_{i=0}^{4} \bar{A} U^{i} \text { and } \bar{A} \cong \mathcal{H}\left(G_{21}\right) .
$$

5. Let

$$
\begin{aligned}
& A:=<S, T \mid \quad \text { STSTSTSTST }=\text { TSTSTSTSTS }, S^{2}=1, \\
& \left(T-y_{0}\right)\left(T-y_{1}\right)\left(T-y_{2}\right)=0>
\end{aligned}
$$

and

$$
\bar{A}:=<T, S T S>\text {. }
$$

Then

$$
A=\bar{A} \oplus S \bar{A}=\bar{A} \oplus \bar{A} S \text { and } \bar{A} \cong \mathcal{H}\left(G_{20}\right) .
$$

6. Let

$$
\begin{aligned}
A:=<S, T \mid & \text { STSTSTSTST }=\operatorname{TSTSTSTSTS~} T^{3}=1, \\
& \left(S-x_{0}\right)\left(S-x_{1}\right)=0>
\end{aligned}
$$

and

$$
\bar{A}:=<S, T S T^{2}, T^{2} S T>.
$$

Then

$$
A=\bigoplus_{i=0}^{2} T^{i} \bar{A}=\bigoplus_{i=0}^{2} \bar{A} T^{i} \text { and } \bar{A} \cong \mathcal{H}\left(G_{22}\right) .
$$

The Schur elements of all irreducible characters of $\mathcal{H}\left(G_{19}\right)$ are calculated in [48. They are obtained via Galois transformations from the following ones:

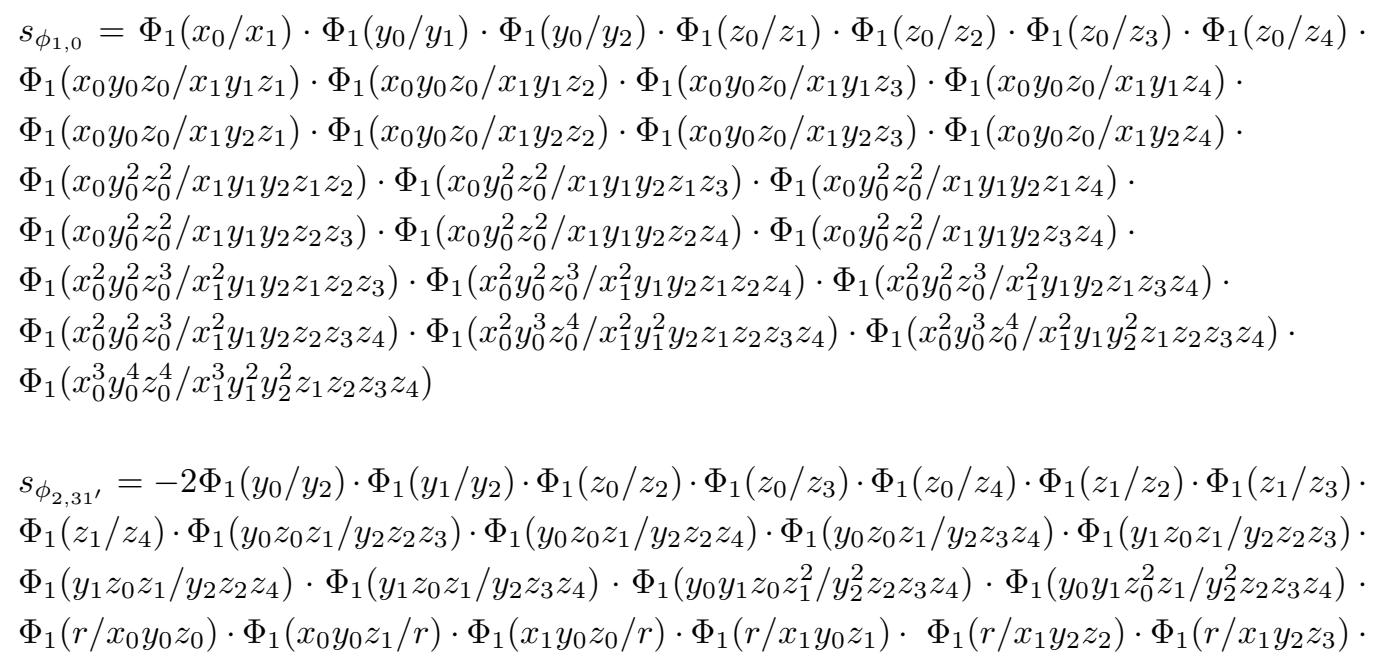


$\Phi_{1}\left(r / x_{1} y_{2} z_{4}\right) \cdot \Phi_{1}\left(r / x_{0} y_{2} z_{2}\right) \cdot \Phi_{1}\left(r / x_{0} y_{2} z_{3}\right) \cdot \Phi_{1}\left(r / x_{0} y_{2} z_{4}\right) \cdot \Phi_{1}\left(r z_{0} z_{1} / x_{0} y_{2} z_{2} z_{3} z_{4}\right) \cdot$

$\Phi_{1}\left(r z_{0} z_{1} / x_{1} y_{2} z_{2} z_{3} z_{4}\right)$

where $r=\sqrt[2]{x_{0} x_{1} y_{0} y_{1} z_{0} z_{1}}$

$s_{\phi_{3,22^{\prime}}}=3 \Phi_{1}\left(x_{1} / x_{0}\right) \cdot \Phi_{1}\left(z_{0} / z_{3}\right) \cdot \Phi_{1}\left(z_{0} / z_{4}\right) \cdot \Phi_{1}\left(z_{1} / z_{3}\right) \cdot \Phi_{1}\left(z_{1} / z_{4}\right) \cdot \Phi_{1}\left(z_{2} / z_{3}\right) \cdot \Phi_{1}\left(z_{2} / z_{4}\right) \cdot$ $\Phi_{1}\left(x_{0} z_{0} z_{1} / x_{1} z_{3} z_{4}\right) \cdot \Phi_{1}\left(x_{0} z_{0} z_{2} / x_{1} z_{3} z_{4}\right) \cdot \Phi_{1}\left(x_{0} z_{1} z_{2} / x_{1} z_{3} z_{4}\right) \cdot \Phi_{1}\left(r / x_{1} y_{0} z_{3}\right) \cdot \Phi_{1}\left(r / x_{1} y_{0} z_{4}\right) \cdot$ $\Phi_{1}\left(r / x_{1} y_{1} z_{3}\right) \cdot \Phi_{1}\left(r / x_{1} y_{1} z_{4}\right) \cdot \Phi_{1}\left(r / x_{1} y_{2} z_{3}\right) \cdot \Phi_{1}\left(r / x_{1} y_{2} z_{4}\right) \cdot \Phi_{1}\left(x_{0} y_{0} z_{0} / r\right) \cdot \Phi_{1}\left(x_{0} y_{0} z_{1} / r\right) \cdot$ $\Phi_{1}\left(x_{0} y_{0} z_{2} / r\right) \cdot \Phi_{1}\left(x_{0} y_{1} z_{0} / r\right) \cdot \Phi_{1}\left(x_{0} y_{1} z_{1} / r\right) \cdot \Phi_{1}\left(x_{0} y_{1} z_{2} / r\right) \cdot \Phi_{1}\left(x_{0} y_{2} z_{0} / r\right) \cdot \Phi_{1}\left(x_{0} y_{2} z_{1} / r\right) \cdot$ $\Phi_{1}\left(x_{0} y_{2} z_{2} / r\right) \cdot \Phi_{1}\left(r^{2} / x_{0} x_{1} y_{0} y_{1} z_{3} z_{4}\right) \cdot \Phi_{1}\left(r^{2} / x_{0} x_{1} y_{0} y_{2} z_{3} z_{4}\right) \cdot \Phi_{1}\left(r^{2} / x_{0} x_{1} y_{1} y_{2} z_{3} z_{4}\right)$ where $r=\sqrt[3]{x_{0}^{2} x_{1} y_{0} y_{1} y_{2} z_{0} z_{1} z_{2}}$

$s_{\phi_{4,18}}=-4 \Phi_{1}\left(y_{1} / y_{0}\right) \cdot \Phi_{1}\left(y_{0} / y_{2}\right) \cdot \Phi_{1}\left(z_{0} / z_{4}\right) \cdot \Phi_{1}\left(z_{1} / z_{4}\right) \cdot \Phi_{1}\left(z_{2} / z_{4}\right) \cdot \Phi_{1}\left(z_{3} / z_{4}\right) \cdot \Phi_{1}\left(x_{0} y_{0} z_{0} / r\right) \cdot$ $\Phi_{1}\left(x_{0} y_{0} z_{1} / r\right) \cdot \Phi_{1}\left(x_{0} y_{0} z_{2} / r\right) \cdot \Phi_{1}\left(x_{0} y_{0} z_{3} / r\right) \cdot \Phi_{1}\left(x_{1} y_{0} z_{0} / r\right) \cdot \Phi_{1}\left(x_{1} y_{0} z_{1} / r\right) \cdot \Phi_{1}\left(x_{1} y_{0} z_{2} / r\right) \cdot$ $\Phi_{1}\left(x_{1} y_{0} z_{3} / r\right) \cdot \Phi_{1}\left(r / x_{0} y_{1} z_{4}\right) \cdot \Phi_{1}\left(r / x_{1} y_{1} z_{4}\right) \cdot \Phi_{1}\left(r / x_{0} y_{2} z_{4}\right) \cdot \Phi_{1}\left(r / x_{1} y_{2} z_{4}\right) \cdot$

$\Phi_{1}\left(r^{2} / x_{0} x_{1} y_{0} y_{1} z_{0} z_{1}\right) \cdot \Phi_{1}\left(r^{2} / x_{0} x_{1} y_{0} y_{1} z_{0} z_{2}\right) \cdot \Phi_{1}\left(x_{0} x_{1} y_{0} y_{1} z_{0} z_{3} / r^{2}\right) \cdot \Phi_{1}\left(x_{0} x_{1} y_{0} y_{1} z_{1} z_{2} / r^{2}\right) \cdot$ $\Phi_{1}\left(r^{2} / x_{0} x_{1} y_{0} y_{1} z_{1} z_{3}\right) \cdot \Phi_{1}\left(r^{2} / x_{0} x_{1} y_{0} y_{1} z_{2} z_{3}\right) \cdot \Phi_{1}\left(r^{2} / x_{0} x_{1} y_{1} y_{2} z_{0} z_{4}\right) \cdot \Phi_{1}\left(r^{2} / x_{0} x_{1} y_{1} y_{2} z_{1} z_{4}\right) \cdot$ $\Phi_{1}\left(r^{2} / x_{0} x_{1} y_{1} y_{2} z_{2} z_{4}\right) \cdot \Phi_{1}\left(r^{2} / x_{0} x_{1} y_{1} y_{2} z_{3} z_{4}\right)$

where $r=\sqrt[4]{x_{0}^{2} x_{1}^{2} y_{0}^{2} y_{1} y_{2} z_{0} z_{1} z_{2} z_{3}}$

$s_{\phi_{5,16}}=5 \Phi_{1}\left(x_{0} / x_{1}\right) \cdot \Phi_{1}\left(y_{2} / y_{0}\right) \cdot \Phi_{1}\left(y_{2} / y_{1}\right) \cdot \Phi_{1}\left(x_{0} y_{0} z_{0} / r\right) \cdot \Phi_{1}\left(x_{0} y_{0} z_{1} / r\right) \cdot \Phi_{1}\left(x_{0} y_{0} z_{2} / r\right) \cdot$ $\Phi_{1}\left(x_{0} y_{0} z_{3} / r\right) \cdot \Phi_{1}\left(x_{0} y_{0} z_{4} / r\right) \cdot \Phi_{1}\left(x_{0} y_{1} z_{0} / r\right) \cdot \Phi_{1}\left(x_{0} y_{1} z_{1} / r\right) \cdot \Phi_{1}\left(x_{0} y_{1} z_{2} / r\right) \cdot \Phi_{1}\left(x_{0} y_{1} z_{3} / r\right) \cdot$ $\Phi_{1}\left(x_{0} y_{1} z_{4} / r\right) \cdot \Phi_{1}\left(r / x_{1} y_{2} z_{0}\right) \cdot \Phi_{1}\left(r / x_{1} y_{2} z_{1}\right) \cdot \Phi_{1}\left(r / x_{1} y_{2} z_{2}\right) \cdot \Phi_{1}\left(r / x_{1} y_{2} z_{3}\right) \cdot \Phi_{1}\left(r / x_{1} y_{2} z_{4}\right) \cdot$ $\Phi_{1}\left(x_{0} x_{1} y_{0} y_{1} z_{0} z_{1} / r^{2}\right) \cdot \Phi_{1}\left(x_{0} x_{1} y_{0} y_{1} z_{0} z_{2} / r^{2}\right) \cdot$

$\Phi_{1}\left(x_{0} x_{1} y_{0} y_{1} z_{0} z_{3} / r^{2}\right) \cdot \Phi_{1}\left(x_{0} x_{1} y_{0} y_{1} z_{0} z_{4} / r^{2}\right) \cdot \Phi_{1}\left(x_{0} x_{1} y_{0} y_{1} z_{1} z_{2} / r^{2}\right) \cdot \Phi_{1}\left(x_{0} x_{1} y_{0} y_{1} z_{1} z_{3} / r^{2}\right) \cdot$ $\Phi_{1}\left(x_{0} x_{1} y_{0} y_{1} z_{1} z_{4} / r^{2}\right) \cdot \Phi_{1}\left(x_{0} x_{1} y_{0} y_{1} z_{2} z_{3} / r^{2}\right) \cdot \Phi_{1}\left(x_{0} x_{1} y_{0} y_{1} z_{2} z_{4} / r^{2}\right) \cdot \Phi_{1}\left(x_{0} x_{1} y_{0} y_{1} z_{3} z_{4} / r^{2}\right)$ where $r=\sqrt[5]{x_{0}^{3} x_{1}^{2} y_{0}^{2} y_{1}^{2} y_{2} z_{0} z_{1} z_{2} z_{3} z_{4}}$

$s_{\phi_{6,15}}=-6 \Phi_{1}\left(z_{0} / z_{1}\right) \cdot \Phi_{1}\left(z_{0} / z_{2}\right) \cdot \Phi_{1}\left(z_{0} / z_{3}\right) \cdot \Phi_{1}\left(z_{0} / z_{4}\right) \cdot \Phi_{1}\left(r / x_{0} y_{0} z_{0}\right) \cdot \Phi_{1}\left(r / x_{0} y_{1} z_{0}\right) \cdot$ $\Phi_{1}\left(r / x_{0} y_{2} z_{0}\right) \cdot \Phi_{1}\left(x_{1} y_{0} z_{0} / r\right) \cdot \Phi_{1}\left(x_{1} y_{1} z_{0} / r\right) \cdot \Phi_{1}\left(x_{1} y_{2} z_{0} / r\right) \cdot \Phi_{1}\left(x_{0} x_{1} y_{0} y_{1} z_{0} z_{1} / r^{2}\right) \cdot$

$\Phi_{1}\left(x_{0} x_{1} y_{0} y_{1} z_{0} z_{2} / r^{2}\right) \cdot \Phi_{1}\left(x_{0} x_{1} y_{0} y_{1} z_{0} z_{3} / r^{2}\right) \cdot \Phi_{1}\left(x_{0} x_{1} y_{0} y_{1} z_{0} z_{4} / r^{2}\right) \cdot \Phi_{1}\left(x_{0} x_{1} y_{0} y_{2} z_{0} z_{1} / r^{2}\right) \cdot$ $\Phi_{1}\left(x_{0} x_{1} y_{0} y_{2} z_{0} z_{2} / r^{2}\right) \cdot \Phi_{1}\left(x_{0} x_{1} y_{0} y_{2} z_{0} z_{3} / r^{2}\right) \cdot \Phi_{1}\left(x_{0} x_{1} y_{0} y_{2} z_{0} z_{4} / r^{2}\right) \cdot \Phi_{1}\left(x_{0} x_{1} y_{1} y_{2} z_{0} z_{1} / r^{2}\right) \cdot$ $\Phi_{1}\left(x_{0} x_{1} y_{1} y_{2} z_{0} z_{2} / r^{2}\right) \cdot \Phi_{1}\left(x_{0} x_{1} y_{1} y_{2} z_{0} z_{3} / r^{2}\right) \cdot \Phi_{1}\left(x_{0} x_{1} y_{1} y_{2} z_{0} z_{4} / r^{2}\right) \cdot$

$\Phi_{1}\left(x_{0}^{2} x_{1} y_{0} y_{1} y_{2} z_{0} z_{1} z_{2} / r^{3}\right) \cdot \Phi_{1}\left(x_{0}^{2} x_{1} y_{0} y_{1} y_{2} z_{0} z_{1} z_{3} / r^{3}\right) \cdot \Phi_{1}\left(x_{0}^{2} x_{1} y_{0} y_{1} y_{2} z_{0} z_{1} z_{4} / r^{3}\right) \cdot$

$\Phi_{1}\left(x_{0}^{2} x_{1} y_{0} y_{1} y_{2} z_{0} z_{2} z_{3} / r^{3}\right) \cdot \Phi_{1}\left(x_{0}^{2} x_{1} y_{0} y_{1} y_{2} z_{0} z_{2} z_{4} / r^{3}\right) \cdot \Phi_{1}\left(x_{0}^{2} x_{1} y_{0} y_{1} y_{2} z_{0} z_{3} z_{4} / r^{3}\right)$ where $r=\sqrt[6]{x_{0}^{3} x_{1}^{3} y_{0}^{2} y_{1}^{2} y_{2}^{2} z_{0}^{2} z_{1} z_{2} z_{3} z_{4}}$

Following Theorem 4.2.4 and [49], Table 8.1, if we set

$$
\begin{array}{ll}
X_{i}^{60}:=\left(\zeta_{2}\right)^{-i} x_{i} & (i=0,1) \\
Y_{j}^{60}:=\left(\zeta_{3}\right)^{-j} y_{j} & (j=0,1,2) \\
Z_{k}^{60}:=\left(\zeta_{5}\right)^{-k} z_{k} & (k=0,1,2,3,4)
\end{array}
$$


then $\mathbb{Q}\left(\zeta_{60}\right)\left(X_{0}, X_{1}, Y_{0}, Y_{1}, Y_{2}, Z_{0}, Z_{1}, Z_{2}, Z_{3}, Z_{4}\right)$ is a splitting field for $\mathcal{H}\left(G_{19}\right)$. Hence, the factorization of the Schur elements over that field is as described by Theorem 4.2.5.

\subsection{The groups $G_{25}, G_{26}$}

The following table gives the specialization of the parameters of the generic Hecke algebra $\mathcal{H}\left(G_{26}\right),\left(x_{0}, x_{1} ; y_{0}, y_{1}, y_{2}\right)$, which gives the generic Hecke algebra of the group $G_{25}$ ([50], Theorem 6.3).

\begin{tabular}{|c|c|c|c|}
\hline Group & Index & $\mathrm{S}$ & $\mathrm{T}$ \\
\hline$G_{26}$ & 1 & $x_{0}, x_{1}$ & $y_{0}, y_{1}, y_{2}$ \\
$G_{25}$ & 2 & $1,-1$ & $y_{0}, y_{1}, y_{2}$ \\
\hline
\end{tabular}

Specialization of the parameters for $\mathcal{H}\left(G_{26}\right)$

Lemma 6.4.1 The algebra $\mathcal{H}\left(G_{26}\right)$ specialized via

$$
\left(x_{0}, x_{1} ; y_{0}, y_{1}, y_{2}\right) \mapsto\left(1,-1 ; y_{0}, y_{1}, y_{2}\right)
$$

is the twisted symmetric algebra of the cyclic group $C_{2}$ over the symmetric subalgebra $\mathcal{H}\left(G_{25}\right)$ with parameters $\left(y_{0}, y_{1}, y_{2}\right)$.

Proof: We have

$$
\begin{aligned}
\mathcal{H}\left(G_{26}\right)=<S, T, U \mid & S T S T=T S T S, U T U=T U T, S U=U S, \\
& \left(S-x_{0}\right)\left(S-x_{1}\right)=0 \\
& \left(T-y_{0}\right)\left(T-y_{1}\right)\left(T-y_{2}\right)=0, \\
& \left(U-y_{0}\right)\left(U-y_{1}\right)\left(U-y_{2}\right)=0>.
\end{aligned}
$$

Let

$$
\begin{aligned}
A:=<S, T, U \mid & S T S T=T S T S, U T U=T U T, S U=U S, S^{2}=1 \\
& \left(T-y_{0}\right)\left(T-y_{1}\right)\left(T-y_{2}\right)=0 \\
& \left(U-y_{0}\right)\left(U-y_{1}\right)\left(U-y_{2}\right)=0>
\end{aligned}
$$

and

$$
\bar{A}:=<S U S, T, U>
$$

Then

$$
A=\bar{A} \oplus S \bar{A}=\bar{A} \oplus \bar{A} S \text { and } \bar{A} \cong \mathcal{H}\left(G_{25}\right) .
$$


The Schur elements of all irreducible characters of $\mathcal{H}\left(G_{26}\right)$ are calculated in [50]. They are obtained via Galois transformations from the following ones:

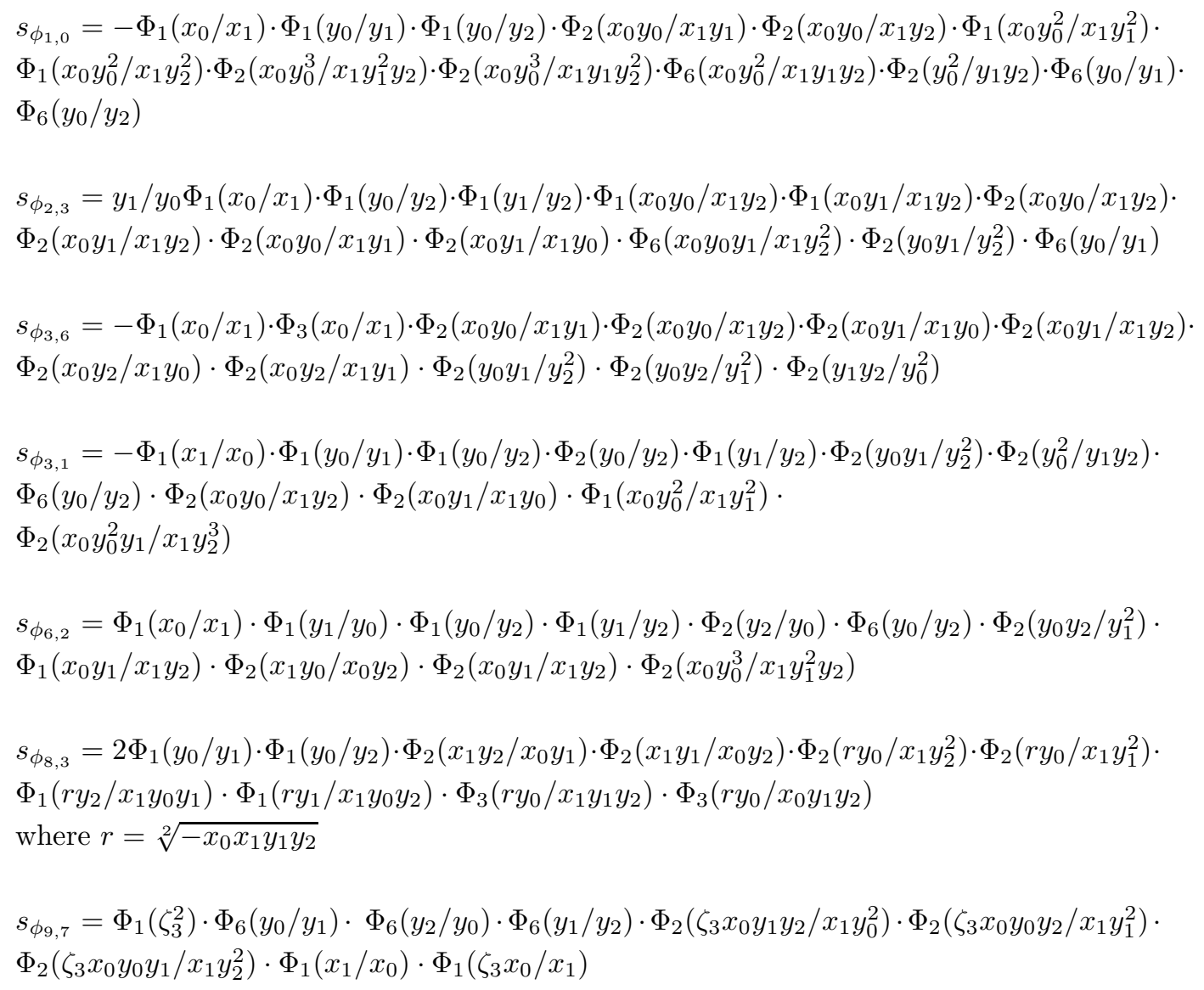

Following Theorem 4.2.4 and [49], Table 8.2, if we set

$$
\begin{array}{ll}
X_{i}^{6}:=\left(\zeta_{2}\right)^{-i} x_{i} & (i=0,1), \\
Y_{j}^{6}:=\left(\zeta_{3}\right)^{-j} y_{j} & (j=0,1,2),
\end{array}
$$

then $\mathbb{Q}\left(\zeta_{3}\right)\left(X_{1}, X_{2}, Y_{1}, Y_{2}, Y_{2}\right)$ is a splitting field for $\mathcal{H}\left(G_{26}\right)$. Hence, the factorization of the Schur elements over that field is as described by Theorem 4.2.5. 


\subsection{The group $G_{28}\left(" F_{4}\right.$ ")}

Let $\mathcal{H}\left(G_{28}\right)$ be the generic Hecke algebra of the real reflection group $G_{28}$ over the ring $\mathbb{Z}\left[x_{0}^{ \pm}, x_{1}^{ \pm}, y_{0}^{ \pm}, y_{1}^{ \pm}\right]$. We have

$$
\begin{aligned}
\mathcal{H}\left(G_{28}\right)=<S_{1}, S_{2}, T_{1}, T_{2} \mid & S_{1} S_{2} S_{1}=S_{2} S_{1} S_{2}, T_{1} T_{2} T_{1}=T_{2} T_{1} T_{2} \\
& S_{1} T_{1}=T_{1} S_{1}, S_{1} T_{2}=T_{2} S_{1}, S_{2} T_{2}=T_{2} S_{2} \\
& S_{2} T_{1} S_{2} T_{1}=T_{1} S_{2} T_{1} S_{2} \\
& \left(S_{i}-x_{0}\right)\left(S_{i}-x_{1}\right)=\left(T_{i}-y_{0}\right)\left(T_{i}-y_{1}\right)=0>
\end{aligned}
$$

The Schur elements of all irreducible characters of $\mathcal{H}\left(G_{28}\right)$ have been calculated in [46]. They are obtained via Galois transformations from the following ones:

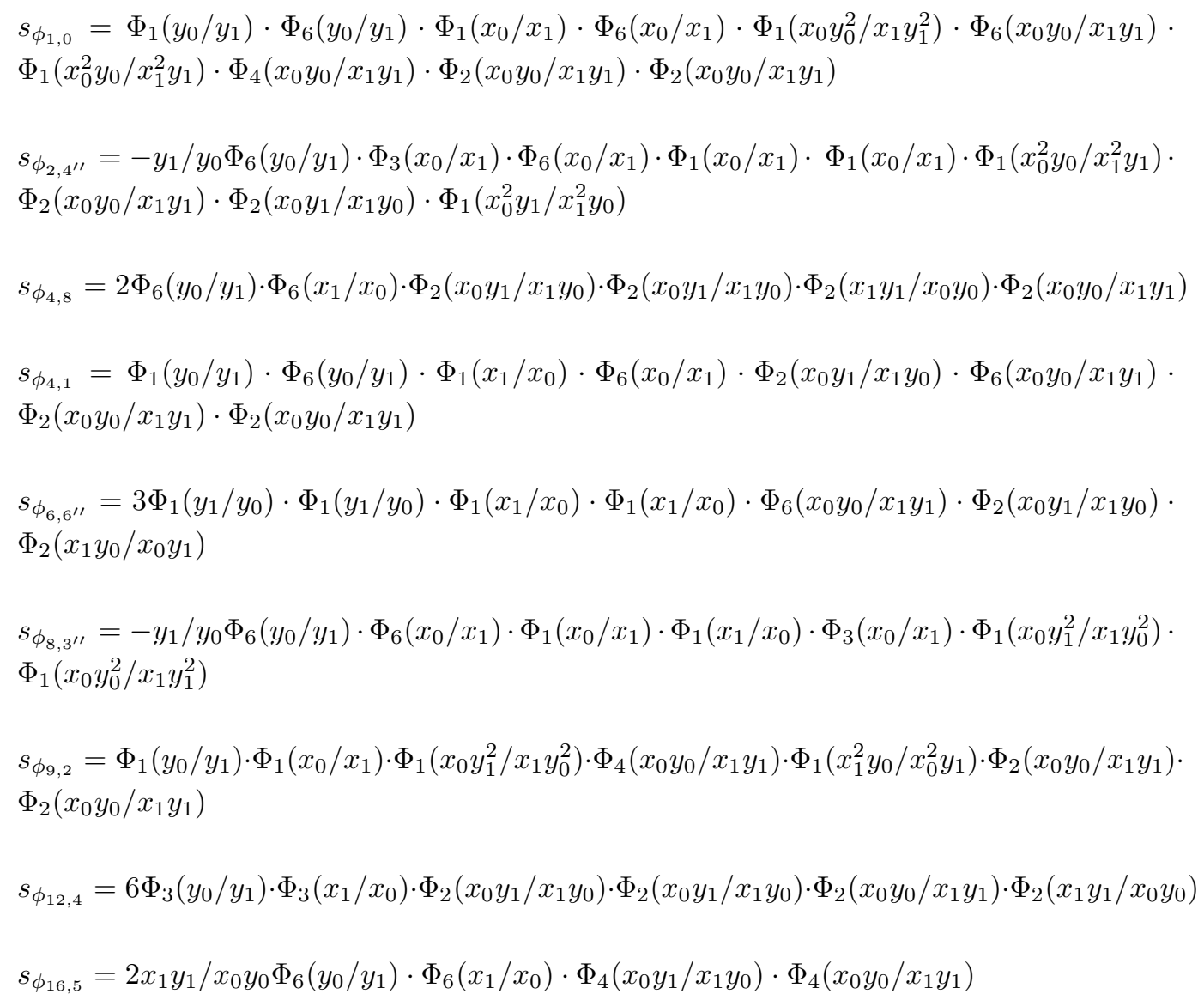

Following Theorem 4.2.4, if we set

$$
\begin{array}{ll}
X_{i}^{2}:=\left(\zeta_{2}\right)^{-i} x_{i} & (i=0,1), \\
Y_{j}^{2}:=\left(\zeta_{2}\right)^{-j} y_{j} & (j=0,1),
\end{array}
$$


then $\mathbb{Q}\left(X_{0}, X_{1}, Y_{0}, Y_{1}\right)$ is a splitting field for $\mathcal{H}\left(G_{28}\right)$. Hence, the factorization of the Schur elements over that field is as described by Theorem 4.2.5.

\subsection{The group $G_{32}$}

Let $\mathcal{H}\left(G_{32}\right)$ be the generic Hecke algebra of the complex reflection group $G_{32}$ over the ring $\mathbb{Z}\left[x_{0}^{ \pm}, x_{1}^{ \pm}, x_{2}^{ \pm}\right]$. We have

$$
\begin{aligned}
\mathcal{H}\left(G_{32}\right)=<S_{1}, S_{2}, S_{3}, S_{4} \mid & S_{i} S_{i+1} S_{i}=S_{i+1} S_{i} S_{i+1} \\
& S_{i} S_{j}=S_{j} S_{i} \text { when }|i-j|>1 \\
& \left(S_{i}-x_{0}\right)\left(S_{i}-x_{1}\right)\left(S_{i}-x_{2}\right)=0>
\end{aligned}
$$

The Schur elements of all irreducible characters of $\mathcal{H}\left(G_{32}\right)$ have been calculated in [50]. They are obtained via Galois transformations from the following ones:

$$
\begin{aligned}
& s_{\phi_{1,0}}=\Phi_{1}\left(x_{0} / x_{2}\right) \cdot \Phi_{1}\left(x_{0} / x_{2}\right) \cdot \Phi_{1}\left(x_{0} / x_{1}\right) \cdot \Phi_{1}\left(x_{0} / x_{1}\right) \cdot \Phi_{1}\left(x_{0}^{3} / x_{1} x_{2}^{2}\right) \cdot \Phi_{1}\left(x_{0}^{3} / x_{1}^{2} x_{2}\right) \cdot \Phi_{1}\left(x_{0}^{5} / x_{1}^{3} x_{2}^{2}\right) \cdot \\
& \Phi_{1}\left(x_{0}^{5} / x_{1}^{2} x_{2}^{3}\right) \cdot \Phi_{2}\left(x_{0}^{4} / x_{1} x_{2}^{3}\right) \cdot \Phi_{2}\left(x_{0}^{4} / x_{1}^{3} x_{2}\right) \cdot \Phi_{2}\left(x_{0}^{2} / x_{1} x_{2}\right) \cdot \Phi_{2}\left(x_{0}^{2} / x_{1} x_{2}\right) \cdot \Phi_{6}\left(x_{0} / x_{2}\right) \cdot \Phi_{6}\left(x_{0} / x_{1}\right) \text {. } \\
& \Phi_{6}\left(x_{0}^{3} / x_{1} x_{2}^{2}\right) \cdot \Phi_{6}\left(x_{0}^{3} / x_{1}^{2} x_{2}\right) \cdot \Phi_{6}\left(x_{0}^{2} / x_{1} x_{2}\right) \cdot \Phi_{4}\left(x_{0}^{2} / x_{1} x_{2}\right) \cdot \Phi_{4}\left(x_{0} / x_{2}\right) \cdot \Phi_{4}\left(x_{0} / x_{1}\right) \cdot \Phi_{3}\left(x_{0}^{2} / x_{1} x_{2}\right) \text {. } \\
& \Phi_{10}\left(x_{0} / x_{2}\right) \cdot \Phi_{10}\left(x_{0} / x_{1}\right) \cdot \Phi_{5}\left(x_{0}^{2} / x_{1} x_{2}\right) \\
& s_{\phi_{4,1}}=\Phi_{1}\left(x_{0}^{4} / x_{1} x_{2}^{3}\right) \cdot \Phi_{1}\left(x_{0}^{3} / x_{1} x_{2}^{2}\right) \cdot \Phi_{1}\left(x_{0}^{3} / x_{1}^{2} x_{2}\right) \cdot \Phi_{1}\left(x_{0}^{2} x_{1} / x_{2}^{3}\right) \cdot \Phi_{1}\left(x_{1} / x_{0}\right) \cdot \Phi_{1}\left(x_{1} / x_{2}\right) \cdot \\
& \Phi_{1}\left(x_{0} / x_{2}\right) \cdot \Phi_{1}\left(x_{0} / x_{2}\right) \cdot \Phi_{2}\left(x_{0}^{5} / x_{1} x_{2}^{4}\right) \cdot \Phi_{2}\left(x_{0}^{3} x_{1} / x_{2}^{4}\right) \cdot \Phi_{2}\left(x_{0}^{3} / x_{1} x_{2}^{2}\right) \cdot \Phi_{2}\left(x_{0}^{2} / x_{1} x_{2}\right) \cdot \Phi_{2}\left(x_{0} / x_{2}\right) \cdot \\
& \Phi_{2}\left(x_{0} x_{1} / x_{2}^{2}\right) \cdot \Phi_{6}\left(x_{0} / x_{2}\right) \cdot \Phi_{6}\left(x_{0} / x_{1}\right) \cdot \Phi_{4}\left(x_{0} / x_{2}\right) \cdot \Phi_{3}\left(x_{0}^{2} / x_{1} x_{2}\right) \cdot \Phi_{10}\left(x_{0} / x_{1}\right) \cdot \Phi_{15}\left(x_{0} / x_{2}\right) \\
& s_{\phi_{5,4}}=\Phi_{1}\left(x_{0}^{3} x_{1}^{2} / x_{2}^{5}\right) \cdot \Phi_{1}\left(x_{0}^{2} x_{1} / x_{2}^{3}\right) \cdot \Phi_{1}\left(x_{0} / x_{2}\right) \cdot \Phi_{1}\left(x_{0} / x_{2}\right) \cdot \Phi_{1}\left(x_{1} / x_{0}\right) \cdot \Phi_{1}\left(x_{1} / x_{0}\right) \cdot \Phi_{1}\left(x_{1} / x_{2}\right) \cdot \\
& \Phi_{1}\left(x_{1} / x_{2}\right) \cdot \Phi_{2}\left(x_{0}^{3} / x_{1} x_{2}^{2}\right) \cdot \Phi_{2}\left(x_{0} x_{1}^{2} / x_{2}^{3}\right) \cdot \Phi_{2}\left(x_{1} / x_{2}\right) \cdot \Phi_{2}\left(x_{0}^{2} / x_{1} x_{2}\right) \cdot \Phi_{2}\left(x_{0} x_{1} / x_{2}^{2}\right) \cdot \Phi_{2}\left(x_{0}^{4} x_{1} / x_{2}^{5}\right) \cdot \\
& \Phi_{2}\left(x_{0} / x_{2}\right) \cdot \Phi_{2}\left(x_{0} / x_{2}\right) \cdot \Phi_{6}\left(x_{0} / x_{2}\right) \cdot \Phi_{6}\left(x_{0} / x_{2}\right) \cdot \Phi_{6}\left(x_{0} / x_{1}\right) \cdot \Phi_{4}\left(x_{0} / x_{1}\right) \cdot \Phi_{3}\left(x_{0} x_{1} / x_{2}^{2}\right) \cdot \\
& \Phi_{12}\left(x_{0} / x_{2}\right) \\
& s_{\phi_{6,8}}=x_{1}^{2} / x_{0}^{2} \Phi_{1}\left(x_{0} / x_{1}\right) \cdot \Phi_{1}\left(x_{1} / x_{0}\right) \cdot \Phi_{1}\left(x_{0} / x_{2}\right) \cdot \Phi_{1}\left(x_{1} / x_{2}\right) \cdot \Phi_{1}\left(x_{0} / x_{2}\right) \cdot \Phi_{1}\left(x_{1} / x_{2}\right) \cdot \\
& \Phi_{1}\left(x_{0} x_{1}^{2} / x_{2}^{3}\right) \cdot \Phi_{1}\left(x_{0}^{2} x_{1} / x_{2}^{3}\right) \cdot \Phi_{2}\left(x_{0} x_{1}^{2} / x_{2}^{3}\right) \cdot \Phi_{2}\left(x_{0}^{2} x_{1} / x_{2}^{3}\right) \cdot \Phi_{2}\left(x_{0}^{2} / x_{1} x_{2}\right) \cdot \Phi_{2}\left(x_{1}^{2} / x_{0} x_{2}\right) \cdot \\
& \Phi_{2}\left(x_{0} x_{1} / x_{2}^{2}\right) \cdot \Phi_{2}\left(x_{0} x_{1} / x_{2}^{2}\right) \cdot \Phi_{2}\left(x_{1} / x_{2}\right) \cdot \Phi_{2}\left(x_{0} / x_{2}\right) \cdot \Phi_{6}\left(x_{0} x_{1} / x_{2}^{2}\right) \cdot \Phi_{6}\left(x_{0} / x_{2}\right) \cdot \Phi_{6}\left(x_{1} / x_{2}\right) \cdot \\
& \Phi_{10}\left(x_{0} / x_{1}\right) \cdot \Phi_{5}\left(x_{0} x_{1} / x_{2}^{2}\right) \\
& s_{\phi_{10,2}}=\Phi_{1}\left(x_{0}^{2} x_{1} / x_{2}^{3}\right) \cdot \Phi_{1}\left(x_{0}^{3} / x_{1} x_{2}^{2}\right) \cdot \Phi_{1}\left(x_{1} / x_{2}\right) \cdot \Phi_{1}\left(x_{1} / x_{2}\right) \cdot \Phi_{1}\left(x_{1} / x_{0}\right) \cdot \Phi_{1}\left(x_{2} / x_{0}\right) \cdot \Phi_{1}\left(x_{0} / x_{2}\right) \cdot \\
& \Phi_{1}\left(x_{0} / x_{2}\right) \cdot \Phi_{2}\left(x_{0}^{4} / x_{1}^{3} x_{2}\right) \cdot \Phi_{2}\left(x_{0}^{3} / x_{1} x_{2}^{2}\right) \cdot \Phi_{2}\left(x_{0} x_{1} / x_{2}^{2}\right) \cdot \Phi_{2}\left(x_{0} x_{2} / x_{1}^{2}\right) \cdot \Phi_{2}\left(x_{0} / x_{2}\right) \cdot \Phi_{2}\left(x_{0} / x_{2}\right) \cdot \\
& \Phi_{2}\left(x_{1} / x_{2}\right) \cdot \Phi_{2}\left(x_{0}^{2} / x_{1} x_{2}\right) \cdot \Phi_{6}\left(x_{0}^{2} x_{1} / x_{2}^{3}\right) \cdot \Phi_{6}\left(x_{0} / x_{2}\right) \cdot \Phi_{6}\left(x_{0} / x_{1}\right) \cdot \Phi_{4}\left(x_{0} / x_{2}\right) \cdot \Phi_{3}\left(x_{0}^{2} / x_{1} x_{2}\right) \\
& s_{\phi_{15,6}}=\Phi_{1}\left(x_{0}^{3} / x_{1} x_{2}^{2}\right) \cdot \Phi_{1}\left(x_{0}^{3} / x_{1}^{2} x_{2}\right) \cdot \Phi_{1}\left(x_{0} / x_{1}\right) \cdot \Phi_{1}\left(x_{0} / x_{1}\right) \cdot \Phi_{1}\left(x_{2} / x_{1}\right) \cdot \Phi_{1}\left(x_{2} / x_{1}\right) \cdot \Phi_{1}\left(x_{2} / x_{0}\right) \cdot \\
& \Phi_{1}\left(x_{0} / x_{2}\right) \cdot \Phi_{2}\left(x_{0}^{3} x_{2} / x_{1}^{4}\right) \cdot \Phi_{2}\left(x_{0}^{3} x_{1} / x_{2}^{4}\right) \cdot \Phi_{2}\left(x_{1}^{2} / x_{0} x_{2}\right) \cdot \Phi_{2}\left(x_{0} x_{1} / x_{2}^{2}\right) \cdot \Phi_{2}\left(x_{0} / x_{2}\right) \cdot \Phi_{2}\left(x_{0} / x_{1}\right) \cdot
\end{aligned}
$$


$\Phi_{2}\left(x_{0}^{2} / x_{1} x_{2}\right) \cdot \Phi_{2}\left(x_{0}^{2} / x_{1} x_{2}\right) \cdot \Phi_{6}\left(x_{0}^{2} / x_{1} x_{2}\right) \cdot \Phi_{6}\left(x_{0} / x_{1}\right) \cdot \Phi_{6}\left(x_{0} / x_{2}\right) \cdot \Phi_{4}\left(x_{0}^{2} / x_{1} x_{2}\right)$

$s_{\phi_{15,8}}=\Phi_{1}\left(x_{1}^{2} x_{2} / x_{0}^{3}\right) \cdot \Phi_{1}\left(x_{0}^{2} x_{2} / x_{1}^{3}\right) \cdot \Phi_{1}\left(x_{0} / x_{2}\right) \cdot \Phi_{1}\left(x_{0} / x_{2}\right) \cdot \Phi_{1}\left(x_{1} / x_{2}\right) \cdot \Phi_{1}\left(x_{1} / x_{2}\right) \cdot \Phi_{1}\left(x_{1} / x_{0}\right) \cdot$ $\Phi_{1}\left(x_{0} / x_{1}\right) \cdot \Phi_{2}\left(x_{1} x_{2} / x_{0}^{2}\right) \cdot \Phi_{2}\left(x_{0} x_{2} / x_{1}^{2}\right) \cdot \Phi_{2}\left(x_{0} x_{1} / x_{2}^{2}\right) \cdot \Phi_{2}\left(x_{0} x_{1} / x_{2}^{2}\right) \cdot \Phi_{2}\left(x_{1} / x_{2}\right) \cdot \Phi_{2}\left(x_{1} / x_{2}\right) \cdot$ $\Phi_{2}\left(x_{0} / x_{2}\right) \cdot \Phi_{2}\left(x_{0} / x_{2}\right) \cdot \Phi_{6}\left(x_{0} / x_{2}\right) \cdot \Phi_{6}\left(x_{1} / x_{2}\right) \cdot \Phi_{4}\left(x_{0} x_{1} / x_{2}^{2}\right)$

$s_{\phi_{20,3}}=\Phi_{1}\left(x_{0}^{2} x_{2} / x_{1}^{3}\right) \cdot \Phi_{1}\left(x_{0} / x_{1}\right) \cdot \Phi_{1}\left(x_{0} / x_{1}\right) \cdot \Phi_{1}\left(x_{2} / x_{0}\right) \cdot \Phi_{1}\left(x_{0} / x_{2}\right) \cdot \Phi_{1}\left(x_{0}^{4} / x_{1} x_{2}^{3}\right) \cdot \Phi_{1}\left(x_{0}^{3} / x_{1} x_{2}^{2}\right) \cdot$ $\Phi_{1}\left(x_{1} / x_{2}\right) \cdot \Phi_{2}\left(x_{0} x_{1}^{2} / x_{2}^{3}\right) \cdot \Phi_{2}\left(x_{1}^{2} / x_{0} x_{2}\right) \cdot \Phi_{2}\left(x_{0}^{2} / x_{1} x_{2}\right) \cdot \Phi_{2}\left(x_{0} x_{1} / x_{2}^{2}\right) \cdot \Phi_{2}\left(x_{0} / x_{2}\right) \cdot \Phi_{2}\left(x_{2} / x_{0}\right) \cdot$ $\Phi_{6}\left(x_{0}^{3} / x_{1}^{2} x_{2}\right) \cdot \Phi_{6}\left(x_{0} / x_{2}\right) \cdot \Phi_{4}\left(x_{0} / x_{2}\right) \cdot \Phi_{3}\left(x_{0} x_{1} / x_{2}^{2}\right)$

$s_{\phi_{20,5}}=-\Phi_{1}\left(x_{1}^{3} / x_{0}^{2} x_{2}\right) \cdot \Phi_{1}\left(x_{0}^{3} / x_{1}^{2} x_{2}\right) \cdot \Phi_{1}\left(x_{0} x_{1}^{2} / x_{2}^{3}\right) \cdot \Phi_{1}\left(x_{0}^{2} x_{1} / x_{2}^{3}\right) \cdot \Phi_{1}\left(x_{2} / x_{1}\right) \cdot \Phi_{1}\left(x_{2} / x_{1}\right) \cdot$ $\Phi_{1}\left(x_{0} / x_{2}\right) \cdot \Phi_{1}\left(x_{0} / x_{2}\right) \cdot \Phi_{2}\left(x_{1}^{3} / x_{0} x_{2}^{2}\right) \cdot \Phi_{2}\left(x_{0}^{3} / x_{1} x_{2}^{2}\right) \cdot \Phi_{2}\left(x_{0} x_{1} / x_{2}^{2}\right) \cdot \Phi_{2}\left(x_{0} x_{1} / x_{2}^{2}\right) \cdot \Phi_{6}\left(x_{0} x_{1} / x_{2}^{2}\right) \cdot$ $\Phi_{6}\left(x_{1} / x_{0}\right) \cdot \Phi_{6}\left(x_{1} / x_{2}\right) \cdot \Phi_{6}\left(x_{0} / x_{2}\right) \cdot \Phi_{3}\left(x_{0} x_{1} / x_{2}^{2}\right)$

$s_{\phi_{20,7}}=\Phi_{1}\left(x_{0}^{3} x_{1} / x_{2}^{4}\right) \cdot \Phi_{1}\left(x_{0} x_{1}^{2} / x_{2}^{3}\right) \cdot \Phi_{1}\left(x_{1} / x_{0}\right) \cdot \Phi_{1}\left(x_{1} / x_{0}\right) \cdot \Phi_{1}\left(x_{2} / x_{0}\right) \cdot \Phi_{1}\left(x_{0} / x_{2}\right) \cdot \Phi_{1}\left(x_{1} / x_{2}\right) \cdot$ $\Phi_{1}\left(x_{1} / x_{2}\right) \cdot \Phi_{2}\left(x_{0} / x_{2}\right) \cdot \Phi_{2}\left(x_{2} / x_{0}\right) \cdot \Phi_{2}\left(x_{0}^{3} x_{2} / x_{1}^{4}\right) \cdot \Phi_{2}\left(x_{0} x_{1}^{2} / x_{2}^{3}\right) \cdot \Phi_{2}\left(x_{0} x_{1} / x_{2}^{2}\right) \cdot \Phi_{2}\left(x_{1}^{2} / x_{0} x_{2}\right) \cdot$ $\Phi_{6}\left(x_{0} / x_{2}\right) \cdot \Phi_{6}\left(x_{0} / x_{2}\right) \cdot \Phi_{6}\left(x_{1} / x_{2}\right) \cdot \Phi_{3}\left(x_{0}^{2} / x_{1} x_{2}\right)$

$s_{\phi_{20,12}}=2 \Phi_{1}\left(x_{2} / x_{1}\right) \cdot \Phi_{1}\left(x_{1} / x_{2}\right) \cdot \Phi_{1}\left(x_{2} / x_{0}\right) \cdot \Phi_{1}\left(x_{1} / x_{0}\right) \cdot \Phi_{1}\left(x_{2} / x_{0}\right) \cdot \Phi_{1}\left(x_{1} / x_{0}\right) \cdot \Phi_{1}\left(x_{0} / x_{2}\right) \cdot$ $\Phi_{1}\left(x_{0} / x_{1}\right) \cdot \Phi_{2}\left(x_{0} x_{1}^{2} / x_{2}^{3}\right) \cdot \Phi_{2}\left(x_{0} x_{2}^{2} / x_{1}^{3}\right) \cdot \Phi_{2}\left(x_{0}^{2} / x_{1} x_{2}\right) \cdot \Phi_{2}\left(x_{1} x_{2} / x_{0}^{2}\right) \cdot \Phi_{2}\left(x_{0} / x_{1}\right) \cdot \Phi_{2}\left(x_{0} / x_{2}\right) \cdot$ $\Phi_{2}\left(x_{0} / x_{1}\right) \cdot \Phi_{2}\left(x_{0} / x_{2}\right) \cdot \Phi_{6}\left(x_{2} / x_{1}\right) \cdot \Phi_{6}\left(x_{1} / x_{2}\right) \cdot \Phi_{3}\left(x_{0}^{2} / x_{1} x_{2}\right)$

$s_{\phi_{24,6}}=\Phi_{1}\left(x_{1}^{3} / x_{0}^{2} x_{2}\right) \cdot \Phi_{1}\left(x_{2}^{3} / x_{0}^{2} x_{1}\right) \cdot \Phi_{1}\left(x_{0} / x_{1}\right) \cdot \Phi_{1}\left(x_{0} / x_{2}\right) \cdot \Phi_{1}\left(x_{0} / x_{1}\right) \cdot \Phi_{1}\left(x_{0} / x_{2}\right) \cdot$ $\Phi_{1}\left(x_{2} / x_{1}\right) \cdot \Phi_{1}\left(x_{1} / x_{2}\right) \cdot \Phi_{2}\left(x_{0} / x_{1}\right) \cdot \Phi_{2}\left(x_{0} / x_{2}\right) \cdot \Phi_{2}\left(x_{0} x_{2} / x_{1}^{2}\right) \cdot \Phi_{2}\left(x_{0} x_{1} / x_{2}^{2}\right) \cdot \Phi_{6}\left(x_{0} / x_{1}\right) \cdot$ $\Phi_{6}\left(x_{0} / x_{2}\right) \cdot \Phi_{4}\left(x_{0} / x_{1}\right) \cdot \Phi_{4}\left(x_{0} / x_{2}\right) \cdot \Phi_{5}\left(x_{0}^{2} / x_{1} x_{2}\right)$

$s_{\phi_{30,4}}=\Phi_{1}\left(x_{0}^{5} / x_{1}^{3} x_{2}^{2}\right) \cdot \Phi_{1}\left(x_{0} / x_{2}\right) \cdot \Phi_{1}\left(x_{1} / x_{0}\right) \cdot \Phi_{1}\left(x_{1} / x_{0}\right) \cdot \Phi_{1}\left(x_{1} / x_{2}\right) \cdot \Phi_{1}\left(x_{1} / x_{2}\right) \cdot \Phi_{1}\left(x_{0} / x_{2}\right) \cdot$ $\Phi_{1}\left(x_{0} / x_{2}\right) \cdot \Phi_{2}\left(x_{1} / x_{0}\right) \cdot \Phi_{2}\left(x_{1} / x_{2}\right) \cdot \Phi_{2}\left(x_{2} / x_{0}\right) \cdot \Phi_{2}\left(x_{0}^{5} / x_{1} x_{2}^{4}\right) \cdot \Phi_{2}\left(x_{0} x_{2}^{2} / x_{1}^{3}\right) \cdot \Phi_{2}\left(x_{0} / x_{2}\right) \cdot$ $\Phi_{2}\left(x_{0} x_{2} / x_{1}^{2}\right) \cdot \Phi_{2}\left(x_{0}^{2} / x_{1} x_{2}\right) \cdot \Phi_{6}\left(x_{0} / x_{1}\right) \cdot \Phi_{6}\left(x_{1} / x_{2}\right) \cdot \Phi_{6}\left(x_{0} / x_{2}\right) \cdot \Phi_{4}\left(x_{0} / x_{2}\right)$

$s_{\phi_{30,12^{\prime}}}=\Phi_{1}\left(x_{1}^{5} / x_{0}^{3} x_{2}^{2}\right) \cdot \Phi_{1}\left(x_{1} / x_{2}\right) \cdot \Phi_{1}\left(x_{0} / x_{1}\right) \cdot \Phi_{1}\left(x_{0} / x_{1}\right) \cdot \Phi_{1}\left(x_{0} / x_{2}\right) \cdot \Phi_{1}\left(x_{0} / x_{2}\right) \cdot \Phi_{1}\left(x_{1} / x_{2}\right)$. $\Phi_{1}\left(x_{1} / x_{2}\right) \cdot \Phi_{2}\left(x_{0} / x_{1}\right) \cdot \Phi_{2}\left(x_{0} / x_{2}\right) \cdot \Phi_{2}\left(x_{2} / x_{1}\right) \cdot \Phi_{2}\left(x_{1}^{5} / x_{0} x_{2}^{4}\right) \cdot \Phi_{2}\left(x_{1} x_{2}^{2} / x_{0}^{3}\right) \cdot \Phi_{2}\left(x_{1} / x_{2}\right) \cdot$ $\Phi_{2}\left(x_{1} x_{2} / x_{0}^{2}\right) \cdot \Phi_{2}\left(x_{1}^{2} / x_{0} x_{2}\right) \cdot \Phi_{6}\left(x_{1} / x_{0}\right) \cdot \Phi_{6}\left(x_{0} / x_{2}\right) \cdot \Phi_{6}\left(x_{1} / x_{2}\right) \cdot \Phi_{4}\left(x_{1} / x_{2}\right)$

$s_{\phi_{36,5}}=\Phi_{1}\left(1 / \zeta_{3}\right) \cdot \Phi_{1}\left(x_{0} / x_{2}\right) \cdot \Phi_{1}\left(x_{1} / x_{0}\right) \cdot \Phi_{1}\left(\zeta_{3} x_{0}^{2} / x_{1} x_{2}\right) \cdot \Phi_{1}\left(\zeta_{3}^{2} x_{0} x_{2} / x_{1}^{2}\right) \cdot \Phi_{1}\left(x_{2}^{2} / \zeta_{3}^{2} x_{0} x_{1}\right) \cdot$ $\Phi_{2}\left(x_{1} x_{2} / x_{0}^{2}\right) \cdot \Phi_{2}\left(x_{0}^{2} x_{2} / \zeta_{3} x_{1}^{3}\right) \cdot \Phi_{2}\left(\zeta_{3}^{2} x_{0}^{2} x_{1} / x_{2}^{3}\right) \cdot \Phi_{6}\left(x_{0}^{2} / x_{1} x_{2}\right) \cdot \Phi_{6}\left(x_{0} / x_{1}\right) \cdot \Phi_{6}\left(x_{0} / x_{2}\right) \cdot \Phi_{6}\left(x_{1} / x_{2}\right) \cdot$ $\Phi_{5}\left(\zeta_{3} x_{0} / x_{2}\right) \cdot \Phi_{5}\left(\zeta_{3} x_{0} / x_{1}\right)$

$s_{\phi_{40,8}}=\Phi_{1}\left(x_{0}^{3} x_{1}^{2} / x_{2}^{5}\right) \cdot \Phi_{1}\left(x_{1} / x_{0}\right) \cdot \Phi_{1}\left(x_{0} / x_{1}\right) \cdot \Phi_{1}\left(x_{0} / x_{2}\right) \cdot \Phi_{1}\left(x_{2} / x_{0}\right) \cdot \Phi_{1}\left(x_{0} / x_{2}\right) \cdot \Phi_{1}\left(x_{1}^{2} x_{2} / x_{0}^{3}\right) \cdot$ $\Phi_{1}\left(x_{2} / x_{1}\right) \cdot \Phi_{2}\left(x_{0} / x_{1}\right) \cdot \Phi_{2}\left(x_{0} x_{1} / x_{2}^{2}\right) \cdot \Phi_{2}\left(x_{0}^{2} / x_{1} x_{2}\right) \cdot \Phi_{2}\left(x_{0} / x_{2}\right) \cdot \Phi_{6}\left(x_{0} / x_{1}\right) \cdot \Phi_{6}\left(x_{1} / x_{2}\right) \cdot$ $\Phi_{4}\left(x_{0} / x_{1}\right) \cdot \Phi_{4}\left(x_{0} / x_{2}\right) \cdot \Phi_{3}\left(x_{0} x_{2} / x_{1}^{2}\right)$ 


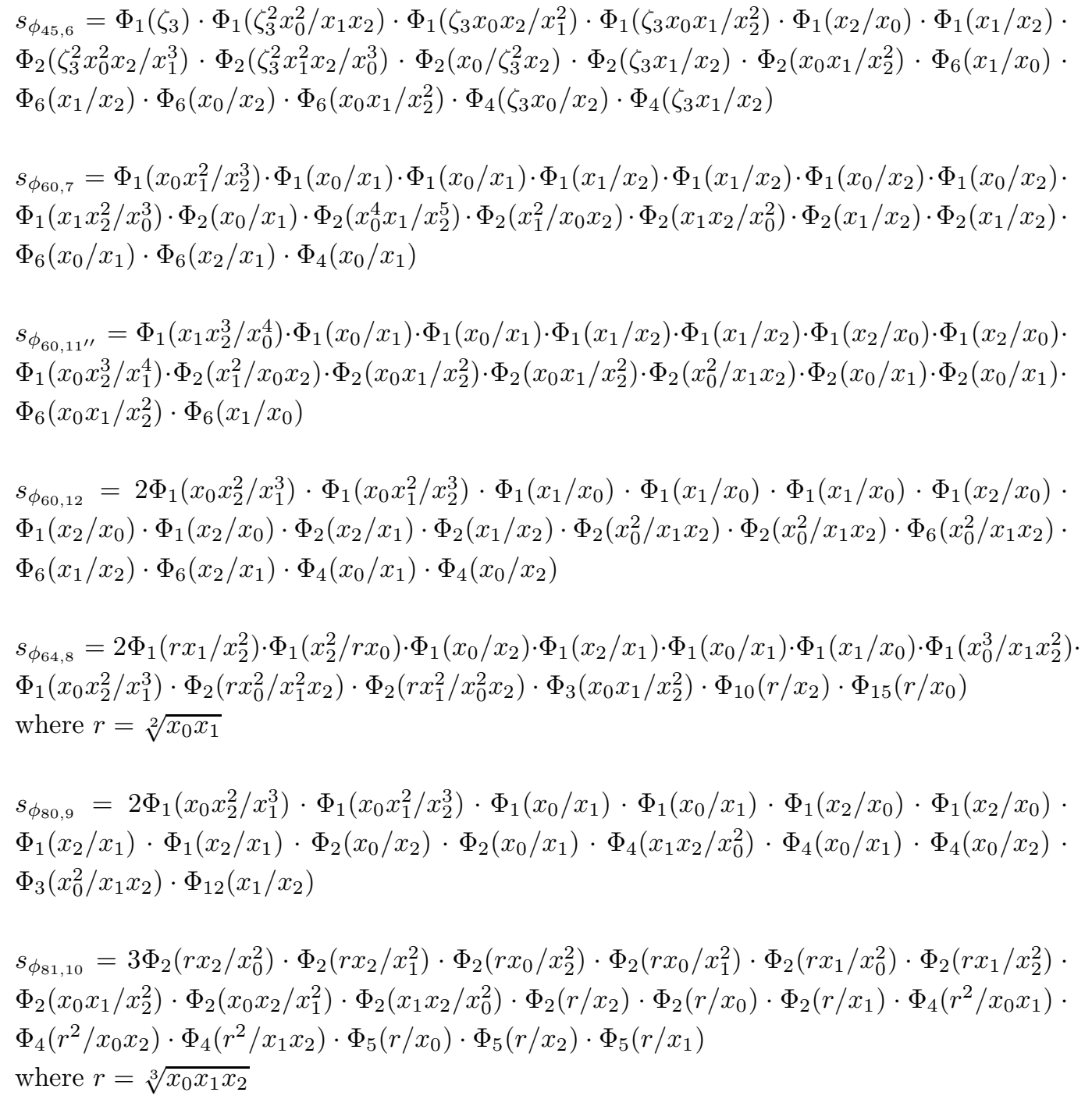

Following Theorem 4.2.4 and [49], Table 8.2, if we set

$$
X_{i}^{6}:=\left(\zeta_{3}\right)^{-i} x_{i}(i=0,1,2),
$$

then $\mathbb{Q}\left(\zeta_{3}\right)\left(X_{0}, X_{1}, X_{2}\right)$ is a splitting field for $\mathcal{H}\left(G_{32}\right)$. Hence, the factorization of the Schur elements over that field is as described by Theorem 4.2.5.

\subsection{The groups $G(d e, e, r)$}

The generic Hecke algebras of the groups $G(d e, e, r)$ are presented in Chapter 5. Here we will only give some applications of Clifford theory and a 
description of their Schur elements.

\subsubsection{The groups $G(d e, e, r), r>2$}

Proposition 1.6 of [2] yields the specialization of the parameters of the generic Hecke algebra $\mathcal{H}(G(d e, 1, r)),\left(x_{0}, x_{1} ; u_{0}, u_{1}, \ldots, u_{d e-1}\right)$, which gives the generic Hecke algebra of the group $G(d e, e, r)$.

Lemma 6.7.1 The algebra $\mathcal{H}(G($ de $, 1, r))$ specialized via

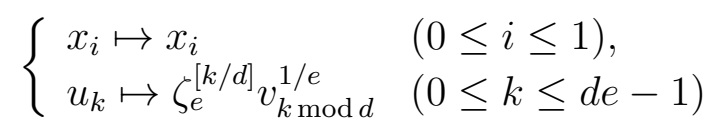

is the twisted symmetric algebra of the cyclic group $C_{e}$ over the symmetric subalgebra $\mathcal{H}(G(d e, e, r))$ with parameters $\left(x_{0}, x_{1} ; v_{0}, v_{1}, \ldots, v_{d-1}\right)$.

Proof: The algebra $\mathcal{H}(G(d e, 1, r))$ is generated by the elements $\mathbf{s}, \mathbf{t}_{1}, \mathbf{t}_{2}, \ldots, \mathbf{t}_{r-1}$ satisfying the relations

- $\mathbf{s t}_{1} \mathbf{s t}_{1}=\mathbf{t}_{1} \mathbf{s t}_{1} \mathbf{s}, \mathbf{s t}_{j}=\mathbf{t}_{j} \mathbf{s}$ for $j \neq 1$,

- $\mathbf{t}_{j} \mathbf{t}_{j+1} \mathbf{t}_{j}=\mathbf{t}_{j+1} \mathbf{t}_{j} \mathbf{t}_{j+1}, \mathbf{t}_{i} \mathbf{t}_{j}=\mathbf{t}_{j} \mathbf{t}_{i}$ for $|i-j|>1$,

- $\left(\mathbf{s}-u_{0}\right)\left(\mathbf{s}-u_{1}\right) \ldots\left(\mathbf{s}-u_{d e-1}\right)=\left(\mathbf{t}_{j}-x_{0}\right)\left(\mathbf{t}_{j}-x_{1}\right)=0$.

Let $A$ be the algebra obtained from $\mathcal{H}(G(d e, 1, r))$ via the given specialization, i.e., the algebra generated by the elements $\mathbf{s}, \mathbf{t}_{1}, \mathbf{t}_{2}, \ldots, \mathbf{t}_{r-1}$ satisfying the same braid relations as above, as well as:

$$
\left(\mathbf{s}^{e}-v_{0}\right)\left(\mathbf{s}^{e}-v_{1}\right) \ldots\left(\mathbf{s}^{e}-v_{d-1}\right)=\left(\mathbf{t}_{j}-x_{0}\right)\left(\mathbf{t}_{j}-x_{1}\right)=0 .
$$

If $\bar{A}:=<\mathbf{s}^{e}, \tilde{\mathbf{t}}_{1}:=\mathbf{s}^{-1} \mathbf{t}_{1} \mathbf{s}, \mathbf{t}_{1}, \mathbf{t}_{2}, \ldots, \mathbf{t}_{r-1}>$, then

$$
A=\bigoplus_{i=0}^{e-1} \mathbf{s}^{i} \bar{A}=\bigoplus_{i=0}^{e-1} \bar{A} \mathbf{s}^{i} \text { and } \bar{A} \cong \mathcal{H}(G(d e, e, r)) .
$$

For $x_{1}=-1$, the algebra $\mathcal{H}(G(d e, 1, r))$ becomes the generic Ariki-Koike algebra $\mathcal{H}_{d e, r}$ associated to $G(d e, 1, r)$. Set $n:=d e$ and $x:=x_{0}$. The following result, which has been obtained independently by Geck, Iancu and Malle ([35]) and by Mathas ([53]), gives a description of the Schur elements of $\mathcal{H}_{n, r}$. Recall that the irreducible characters of $G(n, 1, r)$ are parametrized by the $n$-partitions of $r$. 
Theorem 6.7.2 Let $\lambda$ be an n-partition of $r$ with ordinary standard symbol $B_{\lambda}=\left(B_{\lambda}^{(0)}, B_{\lambda}^{(1)}, \ldots, B_{\lambda}^{(n-1)}\right)$. Fix $L \geq h_{\lambda}$, where $h_{\lambda}$ is the height of $\lambda$. We set $B_{\lambda, L}:=\left(B_{\lambda}^{(0)}\left[L-h_{\lambda}\right], B_{\lambda}^{(1)}\left[L-h_{\lambda}\right], \ldots, B_{\lambda}^{(n-1)}\left[L-h_{\lambda}\right]\right)=\left(B_{\lambda, L}^{(0)}, B_{\lambda, L}^{(1)}, \ldots, B_{\lambda, L}^{(n-1)}\right)$ and $B_{\lambda, L}^{(s)}=\left(b_{1}^{(s)}, b_{2}^{(s)}, \ldots, b_{L}^{(s)}\right)$. Let $a_{L}:=r(n-1)+\left(\begin{array}{c}n \\ 2\end{array}\right)\left(\begin{array}{c}L \\ 2\end{array}\right)$ and $b_{L}:=n L(L-$ $1)(2 n L-n-3) / 12$. Then the Schur element of the irreducible character $\chi_{\lambda}$ is given by the formulae $s_{\lambda}=(-1)^{a_{L}} x^{b_{L}}(x-1)^{-r}\left(u_{0} u_{1} \ldots u_{n-1}\right)^{-r} \nu_{\lambda} / \delta_{\lambda}$, where

$$
\nu_{\lambda}=\prod_{0 \leq s<t<n}\left(u_{s}-u_{t}\right)^{L} \prod_{0 \leq s, t<n} \prod_{b_{s} \in B_{\lambda, L}^{(s)}} \prod_{1 \leq k \leq b_{s}}\left(x^{k} u_{s}-u_{t}\right)
$$

and

$$
\delta_{\lambda}=\prod_{0 \leq s<t<n} \prod_{\left(b_{s}, b_{t}\right) \in B_{\lambda, L}^{(s)} \times B_{\lambda, L}^{(t)}}\left(x^{b_{s}} u_{s}-x^{b_{t}} u_{t}\right) \prod_{0 \leq s<n} \prod_{1 \leq i<j \leq L}\left(x^{b_{i}^{(s)}} u_{s}-x^{b_{j}^{(s)}} u_{s}\right) .
$$

Following [16], Table 1 , the field of definition of $G(n, 1, r)$ is $K:=\mathbb{Q}\left(\zeta_{n}\right)$. By Theorem 4.2.4, if we set

$$
\begin{aligned}
& X^{|\mu(K)|}:=x, \\
& U_{k}^{|\mu(K)|}:=\left(\zeta_{n}\right)^{-k} u_{k} \quad(k=0,1, \ldots, n-1),
\end{aligned}
$$

then the algebra $K\left(X, U_{0}, U_{1}, \ldots, U_{n-1}\right) \mathcal{H}_{n, r}$ is split semisimple. We easily deduce that the factorization of the Schur elements of this algebra is as described by Theorem 4.2.5.

\subsubsection{The groups $G(d e, e, 2), e$ odd}

Lemma 6.7.1 holds when $r=2$ and $e$ is odd.

\subsubsection{The groups $G(d e, e, 2), e$ even}

Suppose that $e=2 f$ for some $f \geq 1$. Proposition 1.6 of [2] yields the specialization of the parameters of the generic Hecke algebra $\mathcal{H}(G(2 f d, 2,2))$, $\left(x_{0}, x_{1} ; y_{0}, y_{1} ; z_{0}, z_{1}, \ldots, z_{f d-1}\right)$, which gives the generic Hecke algebra of the group $G(2 f d, 2 f, 2)$.

Lemma 6.7.3 The algebra $\mathcal{H}(G(2 f d, 2,2))$ specialized via

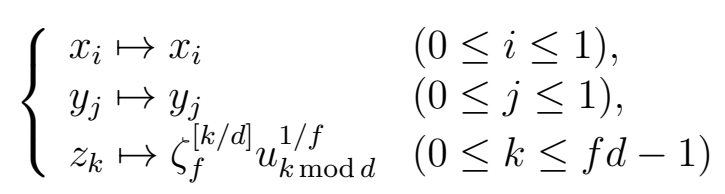

is the twisted symmetric algebra of the cyclic group $C_{f}$ over the symmetric subalgebra $\mathcal{H}(G(2 f d, 2 f, 2))$ with parameters $\left(x_{0}, x_{1} ; y_{0}, y_{1} ; u_{0}, u_{1}, \ldots, u_{d-1}\right)$. 
Proof: We have

$$
\begin{aligned}
\mathcal{H}(G(2 f d, 2,2))=<S, T, U \mid & S T U=T U S=U S T, \\
& \left(S-x_{0}\right)\left(S-x_{1}\right)=0, \\
& \left(T-y_{0}\right)\left(T-y_{1}\right)=0, \\
& \left(U-z_{0}\right)\left(U-z_{1}\right) \ldots\left(U-z_{f d-1}\right)=0>.
\end{aligned}
$$

Let

$$
\begin{aligned}
A:=<S, T, U \mid & S T U=T U S=U S T \\
& \left(S-x_{0}\right)\left(S-x_{1}\right)=0, \\
& \left(T-y_{0}\right)\left(T-y_{1}\right)=0, \\
& \left(U^{f}-u_{0}\right)\left(U^{f}-u_{1}\right) \ldots\left(U^{f}-u_{d-1}\right)=0>
\end{aligned}
$$

and

$$
\bar{A}:=<S, T, U^{f}>
$$

Then

$$
A=\bigoplus_{i=0}^{f-1} U^{i} \bar{A}=\bigoplus_{i=0}^{f-1} \bar{A} U^{i} \text { and } \bar{A} \cong \mathcal{H}(G(2 f d, 2 f, 2)) .
$$

Set $n:=f d=d e / 2$. The group $G(2 n, 2,2)$ has $4 n$ irreducible characters of degree 1 ,

$$
\chi_{i j k}(0 \leq i, j \leq 1)(0 \leq k<n),
$$

and $n^{2}-n$ irreducible characters of degree 2 ,

$$
\chi_{k l}^{1}, \chi_{k l}^{2}(0 \leq k<l<n) .
$$

Following [48, Theorem 3.11, the Schur elements of the irreducible characters of $\mathcal{H}(G(2 n, 2,2))$ are:

$$
\begin{aligned}
& s_{\chi_{i j k}}=\Phi_{1}\left(x_{i} x_{1-i}^{-1}\right) \cdot \Phi_{1}\left(y_{j} y_{1-j}^{-1}\right) \cdot \prod_{l=0, l \neq k}^{n-1}\left(\Phi_{1}\left(z_{k} z_{l}^{-1}\right) \cdot \Phi_{1}\left(x_{i} x_{1-i}^{-1} y_{j} y_{1-j}^{-1} z_{k} z_{l}^{-1}\right)\right) \\
& s_{\chi_{k l}^{1,2}}=-2 \prod_{m=0, m \neq k, l}^{n-1}\left(\Phi_{1}\left(z_{k} z_{m}^{-1}\right) \cdot \Phi_{1}\left(z_{l} z_{m}^{-1}\right)\right) \cdot \prod_{i=0}^{1}\left(\Phi_{1}\left(X_{i} X_{1-i}^{-1} Y_{i} Y_{1-i}^{-1} Z_{k} Z_{l}^{-1}\right) \cdot \Phi_{1}\left(X_{i} X_{1-i}^{-1} Y_{1-i} Y_{i}^{-1} Z_{l} Z_{k}^{-1}\right)\right) \\
& \text { where } X_{i}^{2}:=x_{i}, Y_{j}^{2}:=y_{j}, Z_{k}^{2}:=z_{k} .
\end{aligned}
$$

Following [16], Table 1 , the field of definition of $G(2 n, 2,2)$ is $K:=\mathbb{Q}\left(\zeta_{2 n}\right)$. By Theorem 4.2.4, if we set

$$
\begin{array}{ll}
\mathcal{X}_{i}^{|\mu(K)|}:=\left(\zeta_{2}\right)^{-i} x_{i} & (i=0,1), \\
\mathcal{Y}_{j}^{|\mu(K)|}:=\left(\zeta_{2}\right)^{-j} y_{j} & (j=0,1), \\
\mathcal{Z}_{k}^{|\mu(K)|}:=\left(\zeta_{n}\right)^{-k} z_{k} & (k=0,1, \ldots, n-1),
\end{array}
$$

then the algebra $K\left(\mathcal{X}_{0}, \mathcal{X}_{1}, \mathcal{Y}_{0}, \mathcal{Y}_{1}, \mathcal{Z}_{0}, \mathcal{Z}_{1}, \ldots, \mathcal{Z}_{n-1}\right) \mathcal{H}(G(2 n, 2,2))$ is split semisimple. Hence, the factorization of the Schur elements of this algebra is as described by Theorem 4.2.5. 


\section{Bibliography}

[1] D. Alvis, G. Lusztig, The representations and generic degrees of the Hecke algebra of type $H_{4}$, J. Reine Angew. Math. 336(1982), 201-202.

[2] S. Ariki, Representation theory of a Hecke algebra of $G(r, p, n)$, J. Algebra 177 (1995), 164-185.

[3] S. Ariki, On the semi-simplicity of the Hecke algebra of $(\mathbb{Z} / r \mathbb{Z})>\mathfrak{S}_{n}$, J. Algebra 169, No. 1 (1994), 216-225.

[4] S. Ariki, K. Koike, A Hecke algebra of $(\mathbb{Z} / r \mathbb{Z})$ ? $S_{n}$ and construction of its irreducible representations, Adv. in Math. 106 (1994), 216-243.

[5] M. Benard, Schur indices and splitting fields of the unitary reflection groups, J. Algebra 38(1976), 318-342.

[6] C. T. Benson, The generic degrees of the irreducible characters of $E_{8}$, Comm. Algebra 7(1979), 1199-1209.

[7] D. Bessis, Sur le corps de définition d'un groupe de réflexions complexe, Comm. in Algebra 25 (8) (1997), 2703-2716.

[8] D. Bessis, Zariski theorems and diagrams for braid groups, (2000).

[9] C. Bonnafé, Semicontinuity properties of Kazhdan-Lusztig cells, arXiv:0808.3522.

[10] C. Bonnafé, M. Geck, L. Iancu, T. Lam, On domino insertion and Kazhdan-Lusztig cells in type $B_{n}$ to appear in: Representation theory of algebraic groups and quantum groups (Nagoya, 2006; eds. A. Gyoja et al.), Progress in Math., Birkhäuser.

[11] N. Bourbaki, Éléments de mathématique, Algèbre Commutative, Chap.2, Hermann Ed., Paris, 1961. 
[12] N. Bourbaki, Éléments de mathématique, Algèbre Commutative, Chap.5, Hermann Ed., Paris, 1964.

[13] N. Bourbaki, Éléments de mathématique, Algèbre Commutative, Chap.6, Hermann Ed., Paris, 1964.

[14] N. Bourbaki, Éléments de mathématique, Algèbre Commutative, Chap.7, Hermann Ed., Paris, 1965.

[15] R. Brauer, C. Nesbitt, On the modular representations of groups of finite order, Univ. of Toronto Studies Math. Ser. 4 (1937).

[16] M. Broué, Reflection Groups, Braid Groups, Hecke Algebras, Finite Reductive Groups, Current Developments in Mathematics, 2000, Harvard University and M.I.T. International Press, Boston (2001) 1-103.

[17] M. Broué, On representations of symmetric algebras: An introduction, Notes by Markus Stricker, Forschungsinstitut für Mathematik, ETH, Zürich, 1991.

[18] M. Broué, S. Kim, Familles de caractères des algèbres de Hecke cyclotomiques, Adv. in Mathematics 172(2002), 53-136.

[19] M. Broué, G. Malle, Zyklotomische Heckealgebren, Astérisque 212 (1993), 119-189.

[20] M. Broué, G. Malle, J. Michel, Towards Spetses I, Trans. Groups 4, No. 2-3(1999), 157-218.

[21] M. Broué, G. Malle, R. Rouquier, Complex reflection groups, braid groups, Hecke algebras, J. reine angew. Math. 500 (1998), 127-190.

[22] M. Chlouveraki, Degree and valuation of the Schur elements of cyclotomic Hecke algebras, J. Algebra 320 (2008), 3935-3949.

[23] M. Chlouveraki, GAP functions for Rouquier blocks, http://people.math.jussieu.fr/ chlouveraki.

[24] M. Chlouveraki, Rouquier blocks of the cyclotomic Ariki-Koike algebras, Algebra and Number Theory J., Vol. 2, No. 6 (2008), 689-720.

[25] M. Chlouveraki, Rouquier blocks of the cyclotomic Hecke algebras of $G(d e, e, r)$, to appear in: Nagoya Mathematical Journal, arXiv:0805.0288. 
[26] A. H. Clifford, Representations induced in an invariant subgroup, Ann. of Math. 38 (1937), 533-550.

[27] C. W. Curtis, I.Reiner, Methods of representation theory vol. I, Wiley, New York, 1981; reprinted 1990 as Wiley Classics Library Edition.

[28] E. C. Dade, Compounding Clifford's Theory, Annals of Math., 2nd Series, Vol.91, Issue 1(1970), 236-290.

[29] R. Dipper, A. Mathas, Morita Equivalences of Ariki-Koike algebras, Math. Z. 240 (2002), 579-610.

[30] D. Eisenbud, Commutative Algebra with a View Toward Algebraic Geometry, Springer-Verlag New York, 1995.

[31] W. Feit, The representation theory of finite groups, North-Holland, Amsterdam, 1982.

[32] M. Geck, Beiträge zur Darstellungstheorie von Iwahori-Hecke-Algebren, RWTH Aachen, Habilitations-schrift, 1993.

[33] M. Geck, G. Pfeiffer, Characters of Coxeter groups and Iwahori-Hecke algebras, LMS monographs, New series no. 21, Oxford Univ. Press, 2000.

[34] M. Geck, R. Rouquier, Centers and simple modules for Iwahori-Hecke algebras, Progress in Math. 141, Birkhäuser(1997), 251-272.

[35] M. Geck, L. Iancu, G. Malle, Weights of Markov traces and generic degrees, Indag. Math. 11(2000), 379-397.

[36] M. Geck, G. Hiss, F. Lübeck, G. Malle, G. Pfeiffer, CHEVIE-A system for computing and processing generic character tables, Appl. Algebra Engrg. Comm. Comput. 7 (1996), 175-210.

[37] I. Gordon, M. Martino, Calogero-Moser space, Reduced Rational Cherednik algebras and two-sided cells, arXiv:math.RT/0703153.

[38] J. Graham, G. Lehrer, Cellular algebras, Invent. Math. 123 (1996), 1-34.

[39] J. Guilhot, Kazhdan-Lusztig cells in the affine Weyl groups of rank 2, arXiv:0901.1711.

[40] A. Gyoja, Cells and modular representations of Hecke algebras, Osaka J. Math. 33(1996), 307-341. 
[41] S. Kim, Families of the characters of the cyclotomic Hecke algebras of $G(d e, e, r)$, J. Algebra 289 (2005), 346-364.

[42] S. Lang, Algebra, Springer-Verlag Revised Third Edition, 2002.

[43] G. Lusztig, A class of irreducible representations of a finite Weyl group II, Indag. Math. 44(1982), 219-226.

[44] G. Lusztig, Characters of Reductive Groups over a Finite Field, Annals of Mathematical Studies, Vol. 107, Princeton Univ. Press, Princeton, NJ, 1984.

[45] G. Lusztig, Leading coefficients of character values of Hecke algebras, Proc. Symp. Pure Math., vol. 47(2), Amer. Math. Soc., Providence, RI, 1987, 235-262.

[46] G. Lusztig, Unipotent representations of a finite Chevalley group of type $E_{8}$, Quart. J. Math. Oxford 30(1979), 315-338.

[47] S. Lyle, A. Mathas, Blocks of cyclotomic Hecke algebras, Adv. Math., 216 (2007), 854-878.

[48] G. Malle, Degrés relatifs des algèbres cyclotomiques associées aux groupes de réflexions complexes de dimension deux, Progress in Math. 141, Birkhäuser(1996), 311-332.

[49] G. Malle, On the rationality and fake degrees of characters of cyclotomic algebras, J. Math. Sci. Univ. Tokyo 6(1999), 647-677.

[50] G. Malle, On the generic degrees of cyclotomic algebras, Representation Theory 4(2000), 342-369.

[51] G. Malle, Spetses, ICM II, Doc. Math. J. DMV Extra Volume ICM II(1998), 87-96.

[52] G. Malle, R. Rouquier, Familles de caractères de groupes de réflexions complexes, Representation theory 7(2003), 610-640.

[53] A. Mathas, Matrix units and generic degrees for the Ariki-Koike algebras, J. Algebra 281(2004), 695-730.

[54] J. Michel, The GAP manual including the CHEVIE package, http://people.math.jussieu.fr/ jmichel/htm/index.htm.

[55] M. Nagata, Local rings, Interscience tracts in pure and applied mathematics no.13, Interscience publishers, U.S.A., 1962. 
[56] E. Opdam, A remark on the irreducible characters and fake degrees of finite real reflection groups, Invent. Math. 120 (1995), 447-454.

[57] R. Rouquier, Familles et blocs d'algèbres de Hecke, C. R. Acad. Sciences 329(1999), 1037-1042.

[58] R. Rouquier, q-Schur algebras and complex reflection groups, I, arXiv:math/0509252.

[59] J. P. Serre, Représentations linéaires des groupes finis, Hermann, 1998.

[60] G. C. Shephard, J. A. Todd, Finite unitary reflection groups, Canad. J. Math. 6(1954), 274-304.

[61] D. B. Surowski, Degrees of irreducible characters of $(\mathrm{B}, \mathrm{N})$-pairs of types $E_{6}$ and $E_{7}$, Trans. Amer. Math. Soc. 243(1978), 235-249.

[62] J. Thévenaz, G-algebras and Modular Representation Theory, Oxford Science Publications, Clarendon Press, 1995. 